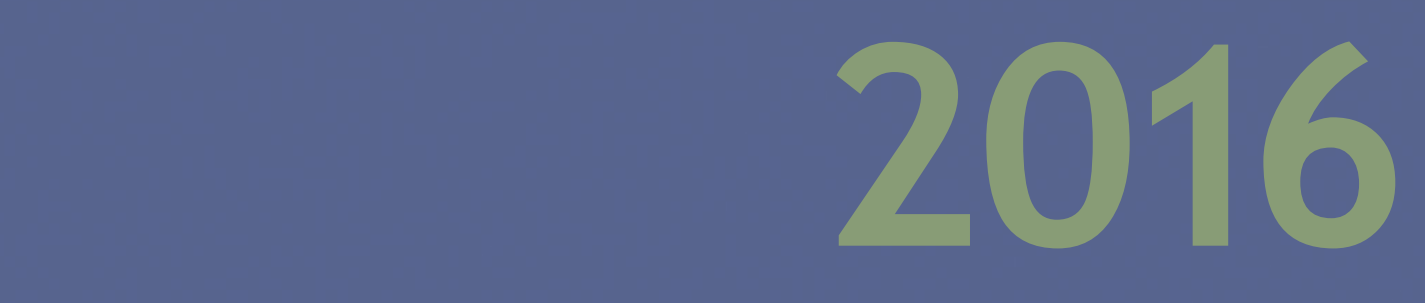

\title{
Health Statistics
} for the Nordic Countries

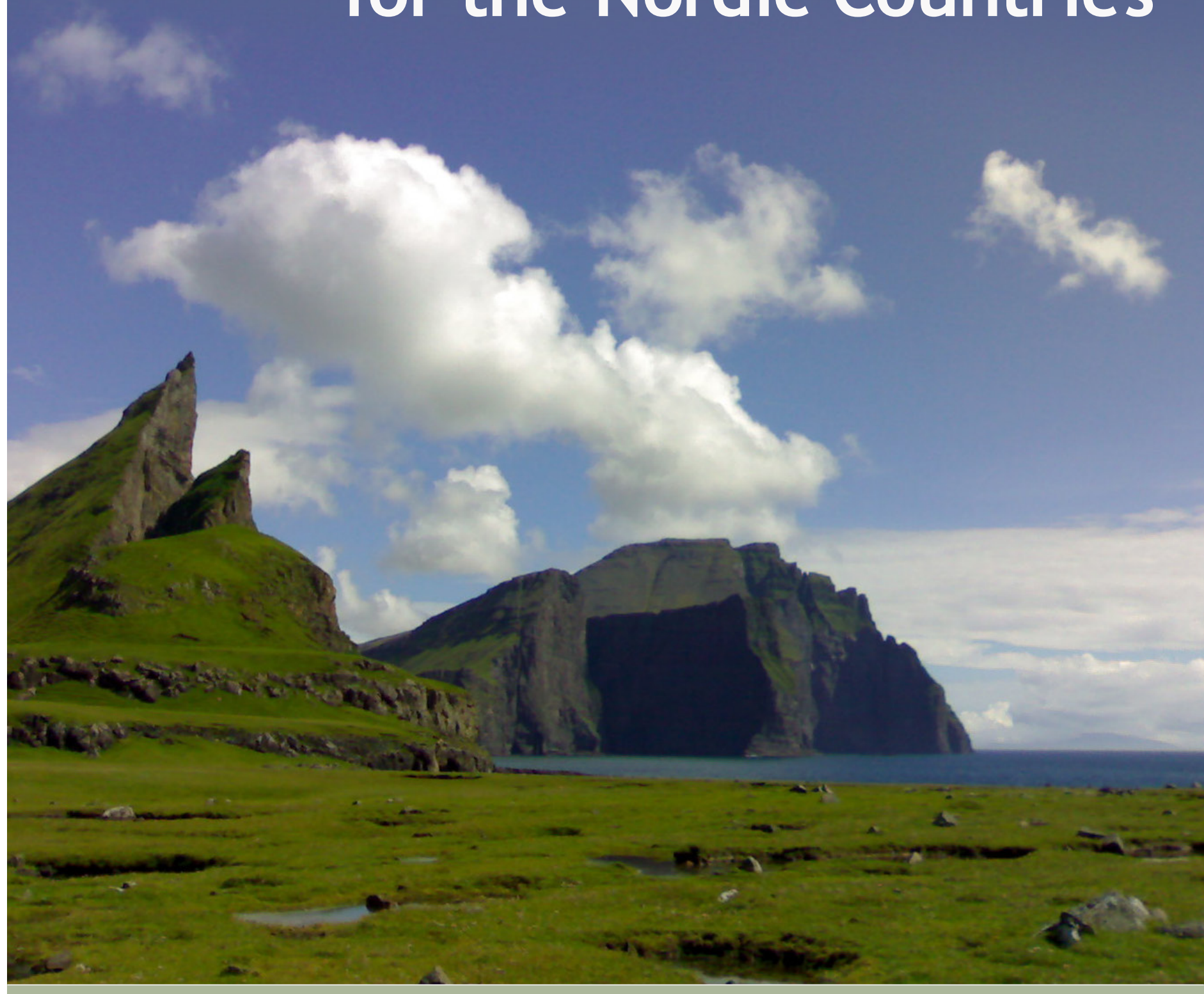



Health Statistics for the Nordic Countries 2016 

Health Statistics for the Nordic Countries 2016 
Members of the Editorial Committee for

Health Statistics for the Nordic Countries

\begin{tabular}{|c|c|}
\hline Denmark & $\begin{array}{l}\text { Linda Saabye Kongerslev } \\
\text { The Danish Health Data Authority }\end{array}$ \\
\hline Faroe Islands & $\begin{array}{l}\text { Petur Joensen } \\
\text { Senior Advisor } \\
\text { Ministry of Health and The Interior }\end{array}$ \\
\hline Greenland & $\begin{array}{l}\text { Henrik Trykker } \\
\text { Chief Medical Officer } \\
\text { National Board of Health }\end{array}$ \\
\hline Finland & $\begin{array}{l}\text { Mika Gissler } \\
\text { Research Professor } \\
\text { National Institute for Health and Wel- } \\
\text { fare THL }\end{array}$ \\
\hline Åland & $\begin{array}{l}\text { Ulla-Liisa Latvala } \\
\text { Inspector, Health Affairs } \\
\text { Government of Aland }\end{array}$ \\
\hline Iceland & $\begin{array}{l}\text { Margrét Björk Svavarsdóttir } \\
\text { Special Advisor } \\
\text { Ministry of Welfare }\end{array}$ \\
\hline Norway & $\begin{array}{l}\text { Jorun Ramm } \\
\text { Statistical Advisor } \\
\text { Statistics Norway }\end{array}$ \\
\hline Sweden & $\begin{array}{l}\text { Ingalill Paulsson Lütz } \\
\text { Researcher } \\
\text { The National Board of Health and Wel- } \\
\text { fare }\end{array}$ \\
\hline \multirow[t]{2}{*}{$\begin{array}{l}\text { NOMESCO's } \\
\text { secretariat }\end{array}$} & $\begin{array}{l}\text { Editor: } \\
\text { Jesper Munk Marcussen }\end{array}$ \\
\hline & $\begin{array}{l}\text { Layout and graphics: } \\
\text { Lene Kokholm }\end{array}$ \\
\hline
\end{tabular}

(c) Nordic Medico-Statistical Committee

Copenhagen 2016

ISBN 978-87-89702-88-9

ISSN 2245-9626 


\section{Contents}

Preface 8

\section{Chapter I}

Organization of the Health Services ............................................ 9

Introduction ...................................................................... 9

1.1 Current and future changes in the health care sector .......................10

1.2 Organization and responsibility for the health sector ....................... 15

1.3 Supervision of health services and health care personnel .....................22

1.4 Complaints about health services and health care personnel .................24

\section{Chapter II}

Population and Fertility 27

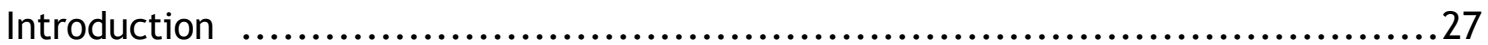

2.1 Population and population trends .........................................27

2.2 Fertility, births, infant mortality and contraception ......................... 37

\section{Chapter III}

Morbidity, Medical Treatment, Accidents and Pharmaceutical Products..............51

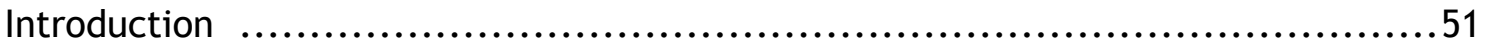

3.1 Diseases related to lifestyle ............................................ 52

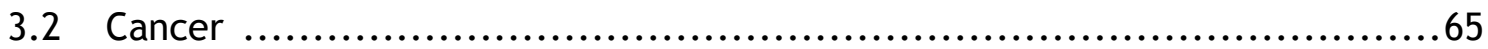

3.3 Immunization schedules .................................................. 77

3.4 Discharges, bed days, average length of stay and patients treated.............79

3.5 Surgical procedures ................................................... 112

3.6 Accidents and self-inflicted injury ...................................... 134

3.7 Development in consumption of pharmaceutical products ................... 135 


\section{Chapter IV}

Mortality and Causes of Death.................................................... 177

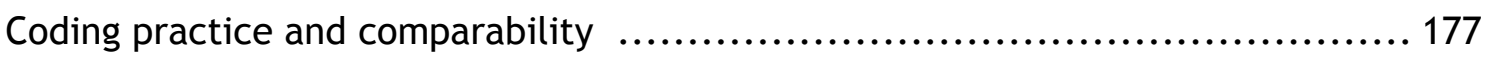

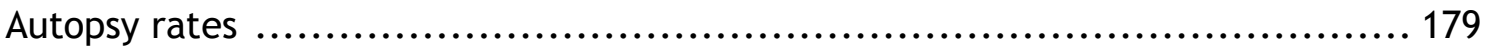

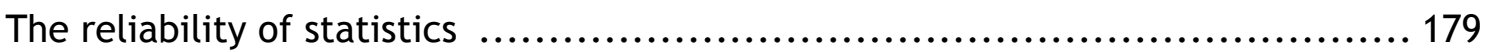

\section{Chapter V}

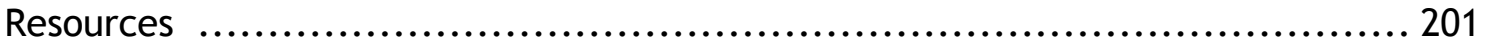

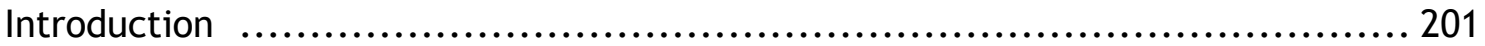

5.1 Financing of health services ........................................... 201

5.2 Charges for health care services per 1 January $2016 \ldots \ldots \ldots \ldots \ldots \ldots \ldots \ldots . . \ldots \ldots . \ldots 203$

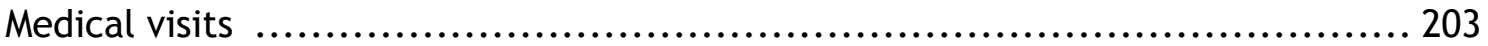

Pharmaceuticals products.................................................. 207

Treatment in hospitals........................................................ 212

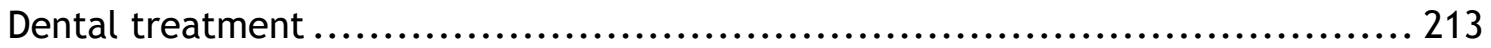

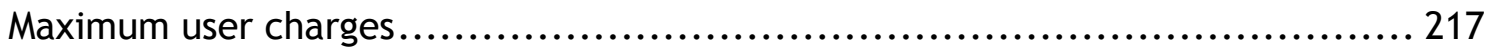

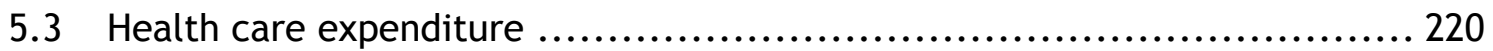

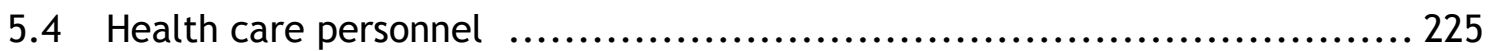

5.5 Capacity and services in the hospital sector ............................. 233

\section{Appendix}

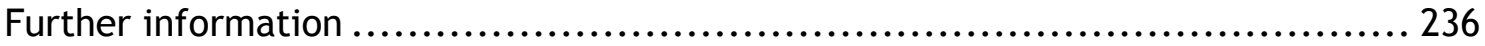

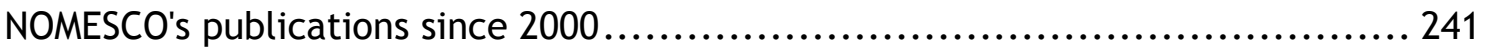




\section{Symbols used in the tables:}

Figures not available or too unreliable for use

Information not applicable

Less than half of the unit used

$0.0 / 0$

Nothing to report (value nil)

Five year averages are always written as 20xx-xy

Two year averages are always written as $20 x x / x y$

Data are always calculated in relation to the respective age groups 


\section{Preface}

The 2016 version of NOMESCO's Health Statistics in the Nordic Countries is now available.

Since 1966, NOMESCO has worked to promote and publish comparable Nordic health statistics. As a permanent part of the work, this annual publication is published with the latest data in the health area.

Health Statistics in the Nordic Countries presents data concerning population trends, illness, hospital treatment and causes of death. Furthermore, a description of the health sector in the Nordic countries, their structure and resources is provided. Health Statistics in the Nordic Countries consequently provides an annual cross section of the health care areas in the Nordic countries.

This version comprises the latest available data as per 1 October 2016. The latest data year may consequently be 2015 or 2014. Previous versions are available at www.nowbase.org, where our database and more specialized publications from projects carried out by NOMESCO can also be found.

Nordic Medico-Statistical Committee (NOMESCO), 14 October 2016 


\section{Chapter 1}

\section{Organization of Health Services}

\section{Introduction}

In the Nordic countries, the health care sector is mainly a public matter.

All the countries have well-established systems of primary health care. In addition to general medical practitioner services, preventive services have been established for mothers and infants, as well as school health care and dental care for children and young people. Preventive occupational health services and general measures for the protection of the environment have also been established in all the countries.

The countries generally have well-developed hospital sectors with highly advanced specialist treatment.

Specialist medical treatment is also offered outside hospitals.

The health services are provided in accordance with legislation, and they are largely financed by public spending or through statutory health insurance schemes. Some patient charges are, however, payable for pharmaceutical products and to some extent also for treatment.

Salary or cash allowances are payable to employees during illness. Self-employed people have the possibility of insuring themselves against illness. 


\subsection{Current and future changes in the health care sector}

\section{DENMARK}

With the Finance Act of 2016, the health sector received a substantial increase in funding of DKK 2.4 billion.

Specific measures are:

- Increased capacity with the aim of maintaining improved patients' rights, including timely assessment and treatment

- Measures for people with dementia and for elderly people receiving medical care

- Improvements to maternity units

- Ensuring that people in all parts of the country receive acute help

In accordance with the Finance Act of 2016, DKK 1 billion will be earmarked each year from 2016 until a policy of dignity for elderly people is widespread and implemented in all the municipalities.

Social Reserve Fund Agreement 2016-2019 (autumn 2015) - National Plan of Action for Dementia 2025

As part of this agreement, DKK 470 million is earmarked for the period 2016-2019 for specific initiatives based on the National Plan of Action for Dementia 2025. The Plan of Action will be finalized in the autumn of 2016. It has three main aims:

- Denmark shall be a dementia-friendly country

- Focus will be directed on the needs and values of people with dementia, coordinated patient pathways with a focus on prevention, early implementation of measures, use of the latest knowledge and more research in this area.

- Relatives shall be actively involved and shall receive more support in their role as a relative.

It is pointed out that the funding in 2016 (DKK 50 million) is included in the total funding of DKK 2.4 billion.

The national aim for the health service - better quality, co-ordination and geographic equality in the health services" (spring 2016)

The Danish Government, the Danish Regions and Local Government Denmark (LGDK) have agreed to develop specific national aims. The eight national aims provide a new opportunity to work with quality and at the same time to move away from requirements related to processes and registration, and to move towards a focus on results for the benefit of individual patients.

Cancer Plan 4 - "Increased measures in the field of cancer" In August 2016, the Danish Government presented the new Cancer Plan 4. The main themes in this plan are:

- A greater focus on patient participation

- Improved survival rates for cancer patients

- Denmark's first smoking-free generation in 2030

With Cancer Plan 4 the Danish Government plans to invest an extra DKK 1.5 billion in the field of cancer in the period 2017-2020. 
Increased measures for elderly people receiving medical care

The Danish Government in co-operation with the Danish People's Party, the Liberal Alliance and the Conservative Party, have allocated DKK 1.2 billion for the period 2016-2019 and then permanently DKK 300 million to prevent excessive occupancy rates in hospitals, and to improve the quality of care and co-operation between different sectors for elderly patients.

It is pointed out that the funding in 2016 (DKK 320 million) is included in the total funding of DKK 2.4 billion.

\section{Committee - for close and co-ordinated health service}

In connection with the financial agreements for 2016, the Local Government Denmark (LGDK) and the Danish Regions have agreed to establish a committee. The committee shall make proposals for a plan to develop a close and co-ordinated health service. The focus is that patient pathways between different sectors shall be co-ordinated, close to the people, cost effective and of high quality.

\section{FAROE ISLANDS}

In May 2012, work on a new Faroese health plan commenced. The purpose of the plan was to find new ways to reorganize the health system and make the health services more efficient. The work aimed at giving priority to preventive measures, and thus to decrease the need for expensive hospitalization and treatment. The new measures were categorized in the following terms: general health promoting measures, earlier and more efficient measures in primary health care, and more focus on improving patients' abilities for self-care especially when coping with chronic illnesses. These measures were described and processed based on the Health Minister's specific request to move the Faroese health care system away from a fragmented system, conceptually as well as in reality, towards a more integrated and holistic health care system.

Since the presentation of the report and the subsequent debate in the Parliament (Løgtingið), which gave the impression that there is great political approval for the solutions stated in the report, the Ministry of Health has worked on implementing several of the new measures. Examples of measures that have already been implemented and measures that are in progress are:

- expansion of the offer of free dental care for children and adolescents

- establishment of local inter-disciplinary health centres

- strengthening initiatives within child and youth psychiatry

- introduction of legal rights for rehabilitation

- offers of special counselling for people with multiple drug use over 75

- establishment of a Public Health Institute

- establishment of health services for people with diabetes and other patients with chronic diseases at local inter-disciplinary health centres

- a National Health Service development plan shall be made as decided by Løgtingið early this summer 


\section{FINLAND}

\section{Social welfare and health care reform}

The Finnish Parliament will soon decide on a government-proposed bill seeking major reform of its social and health care system. The responsibility for service provision will then lie with 18 different regions, which form their own social welfare and health care areas. These local governments will provide the entire chain of social welfare and health care, from physicians taking care of patients to homecare workers caring for the elderly and social workers responsible for child welfare. This system will replace the current decentralized system, when Finland's 313 municipalities are in charge of providing these services. The key objective of the government's proposed reform is to create overseeing regions larger than municipalities responsible for the provision of social welfare and health care services in Finland, so only one administrative body would be in charge of a larger area.

Each region will draw up a region-specific service provision plan with the municipalities and joint authorities in its areas of responsibility. The plan will define how the social welfare and health care services will be carried out. The service provision plans will also determine the quality and level of services and how the availability of and access to the services will be ensured.

According to the government, the reform seeks to mitigate the rising costs of Finland's social welfare and health care services to guarantee that its aging population will continue to receive all of the services it needs in the future. The changes in taxation practices have not been decided yet, but there is a proposal for a new regional tax.

\section{Changes to benefits in 2016}

Reduction of reimbursement rates for the costs of private medical treatment The Government has called for a reduction of EUR 78 million in National Health Insurance payments towards medical care expenses in 2016. The reduction will be implemented by cutting the per-procedure reimbursement rates for the services of physicians, dentists and dental hygienists and the rates for examination and treatment prescribed by a physician or dentist. The rate of reimbursement for oral examinations performed by a dentist and the per-procedure rate for prosthetic dentistry services for veterans will remain at the 2015 level.

New EUR 50 initial deductible introduced for prescription drug reimbursements The Government has introduced an initial deductible of EUR 50 per calendar year that will have to be met before reimbursements for prescription drug expenses will become available. The deductible will not apply to persons under 18 years of age. However, it will count towards the annual maximum on medicine expenses, which will be set at EUR 610.37 in 2016 . The basic rate of reimbursement for prescription medicines will rise from 35 per cent to 40 per cent. The lower and higher special rates of reimbursement (respectively 65 per cent and 100 per cent) will remain unchanged. The per-medicine co-payment for medicines in the higher special reimbursement category will be increased from EUR 3 to EUR 4.50, and the copayment payable after reaching the annual maximum for medicine expenses will be increased from EUR 1.50 to EUR 2.50. 


\section{ICELAND}

A bill on a new reimbursement system for health services was passed as law in June 2016 and will enter into force in February 2017. The main goal is to increase equality between individuals, regardless of health status, and reduce the burden of high expenses for health services through a simpler and more transparent system. The monthly and annual maximum out of pocket payments will be decided by the Minister of Health in a regulation. The patients' payments will depend on total health care costs taken into account during the five previous months. The system is similar to the reimbursement system for pharmaceutical products that was implemented in 2013.

A new Mental Health Policy and a National Mental Health Plan was approved in the Parliament in April 2016. Its main objectives are: Improved well-being and better mental health of the population and active social involvement of individuals who struggle with mental health problems. The plan is for four years and includes an action plan. Part of the action plan is already being implemented.

Directive 2011/24/EU on the application of patients' rights to cross-border healthcare was implemented in June 2016 with Regulation 484/2016 on healthcare received in another country of the EEA agreement and on the role of the national contact point in cross-border health care.

\section{NORWAY}

There are many legal acts in the health field that have been revised or have come into force in recent years: the Public Health Act (folkehelseloven), the Health and Care Services Act (helse- og omsorgstjenesteloven) and the Patients and Users Rights Act (pasient- og brukerrettighetsloven). These acts aim to improve coordination between health care providers, especially between primary health care services provided by the municipality and specialist health services (hospitals). The changes involve issues related to quality of care, patient safety and empowerment of patients.

The Public Health Act came into force on 1 January 2012 and gave a new foundation for strengthening systematic public health work in the development of policies and planning, through better coordination of public health work horizontally across various sectors and actors, and vertically between authorities at the local, regional and national levels. A deliberate substitution policy has been pursued since the late 1980 s, with the aim of replacing relatively expensive inpatient care with less costly out-patient and day care and bringing care closer to patients' homes. With the Coordination Reform (2012) the municipalities are responsible for delivering services for coordinated and integrated pathways, preventive services and early intervention close to where users live.

The Government launched a white paper on primary health and care services in 2015 concerning preventive care, low-threshold services and services for children and young people https://www.regjeringen.no/en/dokumenter/meld.-st.-2620142015/id2409890/. The focus is on aspects related to quality of services, preventive care and cooperation with specialized health services. The goal is to reduce waiting times, increase capacity in the long-term care sector, and to give more priority to better treatment and preventive care within mental health care services and services for people with substance abuse. The goal is also to establish better routines 
for cooperation, both between services at different levels and between services and patients.

\section{Improved patient rights}

In 2015 a new reform of treatment rights within the specialized health services was introduced. The aim is to reduce waiting time, increase freedom of choice for patients and increase efficiency in public hospitals. The right to freedom of choice regarding treatment has been introduced for specialized substance abuse treatment, mental health care and selected areas within somatic health services.

\section{Coordinated services for cancer patients}

In the National Cancer Strategy (Nasjonal kreftstrategi 2013-2017) well-coordinated patient care pathways shall prevent unnecessary waiting time for examination and treatment. Standardized routines for treatment of cancer were introduced in 2015. The goals are to establish centres for diagnosis in all regions and to improve cooperation with GPs.

\section{Knowledge-based health services}

There is a need to use new knowledge and to initiate research for innovation and better practices. The Government will invest in research and innovation through use of quality indicators, data and registers. The goal is that health data should be used in a proactive way, to motivate health personnel to provide better treatment and to improve the quality of services.

\section{Mental health and substance abuse}

The Government will expand services for patients with mental health disorders or substance abuse. An escalation plan has been introduced in 2015 to improve services for these groups, especially in the municipalities. NOK 200 million has been allocated to these services. Another NOK 10 million has been allocated to establish arrangements for more dignified transport of people with mental health disorders.

NOK 200 million has also been allocated to improve school health services and health services provided in health centres.

\section{ICT and digitalization}

Extensive planning and modernizing of the ICT-platform for the whole health and care sector in Norway is taking place. The goal of this work can be summed up thus: one citizen, one medical record. The goal is that health information will follow the patient though the whole patient care pathway. The system of electronic patient journals (EPJ) has been implemented both for general practitioner services and specialized health services. The public dental service and most private dental services also use EPJ. Some municipalities are participating in pilot studies with electronic medical records.

\section{SWEDEN}

The Patients Act (Patientlagen) came into force on 1 January 2015. It is the result of the Patients' Rights Report (Patientmaktutredningen), and shall strengthen the position of patients, and improve patients' integrity, right of self-determination and participation when they receive health care. The Act involves great changes for the 
health service. Residents can now choose the provider of publicly financed primary health services and specialized health services in the whole of Sweden.

The National Board of Health and Welfare has been commissioned by the Government to produce statistics on drug-related mortality and to develop an improved model for monitoring mortality in the future. This task involves using existing health data registers to describe background factors and other relevant information about the group of people who die as the result of drug use or where drugs have contributed to their death. The National Board of Health and Welfare have carried out this task in cooperation with the National Board of Forensic Medicine and the Public Health Agency of Sweden.

As a point of departure for continued development work in the field of eHealth, the Government and the Swedish Association of Local Authorities and Regions have decided to endorse a common vision for eHealth activities until 2025. The vision replaces the latest strategy from 2010, while continuing to build on the ideas and approaches that it contains. The intention is that the vision will be followed by one or more action plans clarifying actions that can contribute to achieving the vision. The concept of eHealth is used in the same way as in the latest strategy, i.e. it covers in a broad sense the use of information and communication technologies in relation to health as defined by the World Health Organization ("a state of complete physical, mental and social well-being"). In this context, the concept of eHealth includes all social services conducted by central or local government or by private actors, all health care and, to the extent relevant, dental care.

\subsection{Organization and responsibility for the health sector}

\section{DENMARK}

Responsibility for health services is relatively decentralized. The main principles are as follows: The State is responsible for legislation, supervision and guidelines. The regions are responsible for hospital services, health insurance and special nursing homes. The municipalities are responsible for primary health care, home nursing, prevention, rehabilitation after hospitalization, and child and school health services. The regional authorities have operational responsibility for health services.

- In principle, primary contact shall always be with a general medical practitioner

- Dental services are provided by private dental practitioners. The services are only a public matter in some dental care services for children

- Health care during pregnancy is the responsibility of the regions

- Child health care is provided according to the Act Relating to Health Visitors and is administered by the municipalities, while health examinations of children are carried out by general medical practitioners

- Home nursing care is provided by the municipalities and is free of charge after referral by a physician

- School and occupational health services are regulated by legislation. Municipalities are responsible for school health services, which are provided by health visitors and physicians 
- Occupational health services are organized by companies and are led by committees with representatives for both employees and employers

- Contact with the health services: As a main rule, patients may contact general medical practitioners, dentists, chiropractors, physiotherapists, chiropodists, psychologists, dental hygienists, emergency wards and emergency and ambulance services without referral

- Public hospitals: Public hospitals are owned by the regions

- Private hospitals: The regions have a contract with some private hospitals to provide treatment under the extended free choice of hospital arrangement. A few private hospitals operate totally independently of the public hospital services. Some specialized hospitals are organized under the hospitals, while others are owned by organizations

- Free choice of hospital: As a rule, patients are free to choose the hospital where they wish to receive treatment

- Practicing specialists: Most practicing specialist physicians work under a contract with the health insurance scheme, and most of their patients are referred from general medical practitioners

- Nursing homes: Ordinary nursing homes are run by the municipalities, but there are many private (independent) nursing homes, which receive residents according to a contract with the municipality in which they are located. Certain specialized nursing homes are run by the regions, for example psychiatric nursing homes

- Pharmacies are organized as private companies, but are subject to government regulation. The state regulates the number and the geographic location of pharmacies, their tasks and the profit margin on pharmaceutical products

\section{FAROE ISLANDS}

The Home Government of the Faroe Islands lays down the rules concerning the tasks, benefits and administration of the health service. The organization of hospital services, specialist fields and primary health services largely follows the Danish system. The same applies to nursing homes, home nursing services, home help services and dental services. Nursing homes, home nursing services and home help services were transferred from the Home Rule Government to the municipalities on 1 January 2015.

Hospital services are run by the Home Rule Government of the Faroe Islands, which defrays all expenditure on operation and maintenance.

All practising physicians are public employees, but they are mainly remunerated by the public health insurance scheme (Heilsutrygd). However, they are also paid directly from the Faroese national budget. Physician services are administered by both the municipal authorities and the state authorities. The municipalities are responsible for properties, inventory and instruments, while the public health insurance scheme stipulates employment conditions and other similar conditions.

The midwifery service is organized under the hospital services.

Physiotherapy services are provided by the public hospital sector and by privately practising physiotherapists.

Pharmacies are run by the public authorities. 


\section{FINLAND}

Municipalities are responsible for health services. The Health Care Act $(1326 / 2011)$ regulates the health care and nursing services that the municipalities are responsible for according to the Public Health Act $(66 / 1972)$ and the Specialist Treatment of Diseases Act (1062/1989). Health care includes measures to promote health and welfare, primary care and specialized nursing.

The municipalities are responsible for the following:

- Guidance and preventive health care, including children's health, health education, counselling concerning contraceptive measures and health surveys and screening

- Medical treatment, including examination and care, medical rehabilitation and first aid

- General medical treatment is provided in health care centres, in In-patient wards or as home nursing

- If a patient's own health centre or hospital cannot provide treatment within the given time, the patient must be offered treatment either in another municipality or at a private institution, without extra cost

- With the exception of emergency cases, patients must be examined and treated within a given period. Patients shall be able to obtain immediate contact with a health centre on weekdays within normal working hours and must have the option of visiting the health centre. If an appointment at a health centre is deemed necessary, patients shall be given an appointment within three working days from the time of contact with the health centre. Normally, treatment is provided at the health centre immediately at the first visit. Treatment that is not provided at the visit shall be started within three months. In cases where health centres provide specialized treatment, the time limits are the same as for specialized health services, i.e. six months. The need for treatment must be assessed within three weeks after referral to a hospital. If a physician has examined a patient and has established that treatment is needed, such treatment shall be started within six months

- Municipalities are also required to provide ambulance services and to ensure that occupational health services are established. Employers can either organize their own occupational health service, or they can enter into an agreement with a health centre or with others who provide occupational health services

- The municipalities must provide services for people with mental illness that can reasonably be offered in health centres

- Children and young people shall receive mental health care within three months if it is assessed to be necessary

- Dental treatment that is assessed to be necessary shall be started within a reasonable time and at the latest within six months

- Dental care includes advice and prevention, dental examination and treatment

- Dental care and treatment paid for by the health insurance scheme is provided for the entire population. Dental care is also provided for adults in health cen- 
tres, particularly in rural municipalities. Most dental treatment for adults is provided by dentists in private practices. Young people under the age of 18 are entitled to dental care free of charge

In many municipalities, social welfare and health services have been integrated in recent years.

\section{ÅLAND}

Due to its home rule, Åland has its own legislation for the health sector, except for administrative interventions in personal freedom, contagious diseases, sterilization, induced abortion, assisted reproduction, forensic medicine and private health care.

The tasks, structure and organization of the public health sector are regulated by the Health Sector Act (2011). Issues that do not fall under the Åland legislation follow Finnish legislation.

All public health services are organized by Åland's Health Care Organization (ÅHS). This organization is governed by a politically elected board.

The Åland Government has overall responsibility for ensuring that the population receives necessary medical care. Primary health services and specialized health services are part of the same organization, ÅHS. In principle, the first contact shall be with the primary health service.

Services that cannot be provided locally are bought from Finland or Sweden, either from private practitioners, private institutions or university hospitals.

The Åland hospitals are specialized institutions that provide both out-patient and In-patient treatment.

Specialists working outside the hospitals can act as consultants for public primary health care services and for private general practitioners.

The structure of primary health care corresponds functionally and ideologically to the Finnish public health care system. Counselling on contraception and maternal and infant health, and school and student health services function as in Finland. Immunization programmes are voluntary and the recommendations are the same as in Finland. Physiotherapy under the ÅHS is a shared function for the primary health service and the hospitals. In addition, a number of private physiotherapists work in the public sector.

Occupational health services are organized in the same way as in Finland.

The public dental service provides dental care for children and young people, and for patient groups that have priority on medical and social grounds. The private sector is well established with a high capacity, and provides an important supplement.

Regulations for pharmacies are the same as in Finland. 


\section{ICELAND}

Responsibility for the health care system is based on a relatively centralized organization. The main principles are as follows:

The Parliament is responsible for the legislation, but the Minister of Health, who is responsible for health care policy in the Ministry of Welfare, is responsible for regulation, supervision and guidelines. The Minister of Health has responsibility for ensuring that all citizens in Iceland have access to optimum health services (primary, secondary and tertiary).

The regional health care institutions are responsible for provision of health services. Health centres provide primary health services, which comprise both prevention and general treatment. Preventive measures include antenatal care, infant health care, school health programmes, immunization, and family planning. Home nursing care is also provided by the health centres, while home help services are provided through the municipal social service system.

As a main rule the first contact should be made at health centres. However, patients can go to specialists and dentists, and can contact emergency and ambulance services without referral.

Medical treatment is largely carried out by practising specialists who work under a contract with the health insurance. Specialists operate in densely populated areas but they also work in health centres in small towns. Specialist treatment is also offered in out-patient wards in hospitals.

Hospital services are provided in three types of facility: 1) specialized hospitals 2) regional hospitals with some specialization and 3) a number of local health care facilities with a few hospital beds but with more long-term beds for elderly people. These hospitals have functions that are similar to nursing homes.

Dental treatment is provided in private dental practices.

Physiotherapy services are provided in health centres, but most treatment in urban areas is provided by private physiotherapists. They have a contract with the health insurance.

Most nursing homes are independent institutions, run by municipalities, voluntary organizations and the like. They are financed partly by user charges, but mainly by health insurance.

According to law, occupational health services are the responsibility of the employer. Larger companies buy these services from practising physicians, consultancy firms, or from health centres.

Pharmacies are organized by the pharmacy owners, in accordance with the legislation. Municipalities have the right to comment on the location of pharmacies but the Medicine Agency regulates their functions.

\section{NORWAY}

The Norwegian health care system is based on the principles of universal access, decentralization and free choice of provider. It is financed by taxation and user charges. All residents are covered by the National Insurance Scheme (Folketrygden, NIS), managed by the Norwegian Health Economics Administration (Helseøkonomiforvaltningen, HELFO). 
While health care policy is controlled centrally, responsibility for provision of health care is decentralized. Local authorities at the municipal level organize and finance primary health care services. The Norwegian Government has overall supervision and financial responsibility for the hospital sector. Norway's four regional health authorities control the provision of specialized health services. Most hospitals in Norway are public hospitals, funded and owned by the state. A small number of hospitals are privately owned. Specialist health services include hospitals for patients with somatic or psychiatric/psychological disorders, out-patient departments, centres for training and rehabilitation, and institutions for drug addicts.

The municipalities are responsible for preventive health and for providing primary health services, while the County Authorities provide dental care. Primary health services are financed through grants from the Norwegian Government, local tax revenues, reimbursements from the National Social Security System, and user charges. All consultations for children under 16 years of age are free.

The public dental health services (PDHS) are administered and funded by the county municipalities. Children under 18 years of age are entitled to free dental treatment except for orthodontic treatment.

All municipalities have an agreement with a Regional Health Authority. When patients are discharged from hospitals, they are followed up by the municipal health services.

\section{SWEDEN}

The State has overall responsibility for health policy, but responsibility for health services is divided between the State, the county authorities and the municipal authorities. Regions are formally county authorities but with an expanded responsibility for regional development.

The Health and Medical Service Act (Hälso- och sjukvårdslagen, HSL) lays down the division of responsibility for health services between the county authorities and the municipal authorities. The Act gives the county authorities and the municipal authorities the task of ensuring that all inhabitants have equal access to sound and adequate services.

The activities of the county councils are mainly financed by county taxes and state grants. Patient charges and other patient contributions make up a small part of the income of the county councils.

The National Board of Health and Welfare, NBHW (Socialstyrelsen) is a government agency under the Ministry of Health and Social Affairs, with many different duties within the fields of social services, health and medical services, patient safety and epidemiology. The National Board of Health and Welfare administers a number of register to be able to analyze and monitor trends in health care and social services. The NBHW works with Regional and local comparisons and Performance Assessments to encourage the providers and management of health care to improve performance.

National guidelines indicate the benefits and risks of different interventions and support health and medical care professionals in prioritising the right interventions for those with the greatest need. The purpose of the guidelines is to ensure that people have access to good health and medical care. 
The Health and Social Care Inspectorate (Inspektionen för vård och omsorg, IVO) is a government agency responsible for supervising health care, social services and activities under the Act, concerning Support and Service for Persons with Certain Functional Impairments (LSS). According to the Swedish Patient Safety Act, all healthcare providers are required to register their activities with the Health and Social Care Inspectorate.

The Medical Products Agency, MPA (Läkemedelsverket) is the Swedish national authority responsible for regulation and surveillance of the development, manufacturing and marketing of drugs and other pharmaceutical products. The Medical Products Agency also maintains supervision over all pharmacies in Sweden. The task is to ensure that both the individual patient and healthcare professionals have access to safe and effective pharmaceutical products and that these are used in a rational and costeffective manner. The agency is also the licensing and regulatory authority for the legal handling of narcotic drugs.

The Dental and Pharmaceutical Benefits Agency (Tandvårds- och läkemedelsförmånsverket, TLV) is a central government agency whose remit is to determine whether a pharmaceutical product, medical device or dental care procedure shall be subsidized by the state. TLV also determines retail margins for all pharmacies in Sweden, regulates the substitution of medicines at the pharmacies and supervises certain areas of the pharmaceutical market.

TLV determines whether licensed pharmaceutical products and extemporaneous medicines (preparations that are tailor-made for a certain patient) will be included in the high-cost threshold. TLV also determines which dental care procedures will be subsidised and sets reference prices, i.e. the prices on which reimbursement is calculated.

The task of the Swedish eHealth Agency (eHälsomyndigheten) is to lead and coordinate government e-health initiatives. All pharmacies in Sweden use the eHealth data base to get the information they need to dispense a prescription.

The Public Health Agency of Sweden (Folkhälsomyndigheten) has responsibility for public health issues, and for ensuring that people have equal opportunities for good health. It does this through monitoring public health, analysing background factors, and evaluating public health initiatives. It also has responsibility for promoting health, preventing illness and aiding the control of infectious diseases through epidemiological and microbiological monitoring.

The Swedish Agency for Health Technology Assessment and Assessment of Social Services (Statens beredning för medicinsk och social utvärdering, SBU) is an independent national authority, given the task by the government of assessing health care interventions from a broad perspective, covering medical, economic, ethical and social aspects.

SBU evaluates the scientific basis for methods currently in use and new methods used in health and social services, and for activities supported by the Act on support and services for certain physically impaired people. SBU also evaluates methods used by medical and social services. SBU assessments are based on 'systematic literature 
reviews' of published research. The review method developed by SBU is thorough and rigorous.

\subsection{Supervision of health services and health care personnel}

In Denmark, supervision of health services is carried out by the Danish Patient Safety Authority with the assistance of the Danish Patient Safety Authority, Supervision and Guidance offices North/South/East. These institutions are independent, politically and administratively, of the regional and municipal health authorities. In this way, the chief medical officers work as independent advisers and supervisors at all levels. Supervision of health care personnel and their professional activities is also carried out by the Danish Patient Safety Authority. Decisions concerning individuals can be appealed to the responsible minister and, if necessary, to the courts.

In the Faroe Islands, the Chief Medical Officer, who is employed by the Danish Ministry of Health, shares responsibility with the Danish Board of Health for supervision of health services. The chief medical officer is the consultant to the Faroese and Danish authorities regarding health matters.

The Office of the Chief Medical Officer is an independent institution under the Government of Greenland and is responsible for supervision of health services in Greenland. The chief medical officer advises and assists the Government of Greenland and other authorities in questions of health.

Supervision of health services in Finland is organized in a less formal way than in the other Nordic countries. Supervisory tasks are spread out in the whole health services system. A nationwide body for the protection of patients' rights has been established. This body may assess whether the services provided by a municipality meet the required standards. If the body finds that the services are inadequate, and that the municipality is responsible for this, it can make recommendations about how the deficiencies may be dealt with, and give a time limit for when improvements shall be made.

Supervision of health care personnel in Åland is carried out according to Finnish law.

In Iceland, The Directorate of Health carries out supervision of health institutions, health care personnel, prescription of pharmaceutical products, measures for combating substance abuse and control of all public health services.

The Icelandic Medicines Agency carries out advisory and supervisory tasks regarding pharmaceutical products to pharmacies, pharmaceutical companies and the public.

In Norway, supervision of health and social services is the responsibility of the Norwegian Board of Health Supervision (centrally) and the Offices of the County Governors in each county. The Norwegian Board of Health Supervision is a national public institution organized under the Ministry of Health and Care Services. The Offices of the County Governors are responsible for supervision of health services and health care personnel at the county level. The legislation provides the framework for supervision of health and care services (Supervision of Health and Care Services Act). The supervision authorities work independently of the political management. 
The Offices of the County Governors deal with complaints against individual health care personnel. Supervision applies to all health services, irrespective of whether they are provided by municipalities, private businesses, publicly owned hospitals or health care personnel who run their own practice. The supervision authorities may find that the statutory requirements have not been met, and give advice on how to make improvements. If there are grounds for more serious sanctions against health care personnel, the case is forwarded to the Norwegian Board of Health Supervision.

In Sweden, the Health and Social Care Inspectorate (IVO) is the national supervising authority for social services and for health services. The purpose of supervision is to ensure that citizens receive social care and health care, which is safe, is of high quality and is carried out in accordance with existing laws and regulations. The Inspectorate's work also includes presenting the supervised organizations with the results of supervision, to provide feedback, advice and guidance regarding the supervision and to ensure that discrepancies and irregularities are corrected.

The Act on patient safety (2010:659) regulates which measures IVO can and must carry out in the supervision of health personnel. If IVO decides upon inspection that health personnel are a danger to patient safety, IVO reports this to the Medical Responsibility Board (Hälso- och sjukvårdens ansvarsnämnd, HSAN), which decides whether authorization to work within the health services shall be with-drawn or limited. 


\subsection{Complaints about health services and health care personnel}

\section{DENMARK}

The National Agency for Patients' Rights and Complaints (Sundhedsvaesenets Disciplinarnaevn) deals with complaints concerning authorized health care personnel. The Danish Safety Authority deals with complaints concerning the place of treatment but can handle complaints against health care personnel and place of treatment. Both the National Agency for Patients' Rights and Complaints (Sundhedsvaesenets Disciplinaernavn) and the Danish Safety Authority can deal with complaints, but complaints cannot be dealt with by both these authorities at the same time.

\section{FAROE ISLANDS}

To a certain extent, the Faroese health system is covered by the regular Danish complaints system. Complaints about health services carried out by authorised health personnel in the Faroe Islands are dealt with by the National Agency for Patients' Rights and Complaints (Sundhedsvaesenets Disciplinaernaevn) in Denmark. Complaints about cases regarding rights of access to patient records are dealt with by the Danish Patient Ombudsman. Complaints about coercion in connection with mental health care are dealt with by the Faroese Psychiatric Complaints Board (Psykiatriska kærunevndin). The decisions of the Complaints Board can be appealed to the Psychiatric Appeals Board in Denmark. Complaints about non-health professional services are dealt with by the Faroese Complaints Board for Social and Health Cases (Kærunevndin í almanna- og heilsumálum), except complaints about the right of access to patient records, which, as already mentioned, are dealt with by the Danish Patient Ombudsman.

Patients who have been referred by the Faroese health care system who receive treatment in the Danish hospital services, are fully covered by the Danish complaints system.

\section{GREENLAND}

Complaints concerning health issues must be addressed in writing to the National Board of Health, which prepares the case and makes recommendations about a decision on the complaint. The cases are then sent to the Danish Patients' Complaints Board where the Disciplinary Board makes a decision about the cases. Complaints concerning services are submitted to the Health Management, and questions concerning compensation are dealt with by the Directorate of Health and Infrastructure.

\section{FINLAND}

Patients have several options when they wish to complain about the treatment or services they have received. The simplest way is to express dissatisfaction to the physician who provided the treatment, or to contact the physician in charge of the hospital department or health centre. If further assistance is needed in order to solve the problem, there are two possibilities. The patient can contact either the Regional State Administrative Agency or the National Supervisory Authority for Welfare and Health (VALVIRA). Both these bodies can give a written expert opinion, or give sanctions if necessary. 


\begin{abstract}
ÅLAND
Complaints concerning treatment must be addressed to the institution providing the treatment, to the national authorities, or to the Åland Government, as in Finland. The Patient Ombudsman is employed by the Åland Government and is thus independent of the treatment institutions. The Patient Ombudsman may take up issues of principal significance with the "Patients Board of Trust" where the issues may be discussed and form the basis for decisions, although the Board cannot make a decision in individual cases.
\end{abstract}

\title{
ICELAND
}

In accordance with the Patients' Rights Act, patients have the right to complain about health services. A patient can direct his complaint to the respective healthcare institution and to the Directorate of Health. Decisions of the Directorate of Health can be appealed to the Minister of health.

\section{NORWAY}

Patients can send complaints about health services to the institution where they were treated or to the municipal board in cases of municipal health services. Alternatively patients can send complaints to the Office of the County Governor. Serious cases are forwarded to the Norwegian Board of Health Supervision. Authorized health care personnel can be given a warning, their right to prescribe addictive drugs can be withdrawn, or their authorization can be withdrawn.

The role of the Health and Social Services Ombudsman is to take care of the needs, interests and statutory rights of patients and clients. The ombudsman can help patients who do not get the treatment they need. There is an ombudsman in each county.

In cases of injury caused during treatment, the patient can apply for compensation to the Norwegian System for Compensation for Injuries to Patients (Norsk Pasientskadeerstatning). Patients with complaints about public and private health services and dental services can apply for compensation.

\section{SWEDEN}

The Health and Social Care Inspectorate (Inspektionen för vård och omsorg, IVO) is a government agency responsible for supervising health care, social services and activities under the Act concerning Support and Service for Persons with Certain Functional Impairments (LSS). IVO is also responsible for issuing certain permits in these areas. Its supervision remit covers the processing of complaints concerning, for example, the reporting of irregularities in health care and social care (called lex Maria and lex Sarah reports) and the municipal obligation to report non-enforced decisions. 
Organization of Health Services 


\section{Chapter 2}

\section{Population and Fertility}

\section{Introduction}

This chapter begins with a general description of the population in the Nordic countries followed by a more detailed description of fertility, births, infant mortality and contraceptive methods.

\subsection{Population and population trends}

The population structure varies somewhat among the Nordic countries, Sweden having the oldest and Greenland the youngest population.

The development in population growth varies somewhat among the Nordic countries. The natural increase has been largest in Iceland, the Faroe Islands and Greenland throughout the past decade. Denmark, Åland and Sweden have had the lowest natural increase. In 2014, net migration contributed to population growth in all the Nordic countries with the exception of Greenland. In addition, there is a large deficit of women of fertile age in the Faroe Islands.

Life expectancy in the Nordic countries has increased significantly, and even though women generally live longer, the difference between the life expectancy of men and of women has been reduced. 
Figure 2.1.1 Mean population by gender and age as a percentage of the total population $2015^{1}$
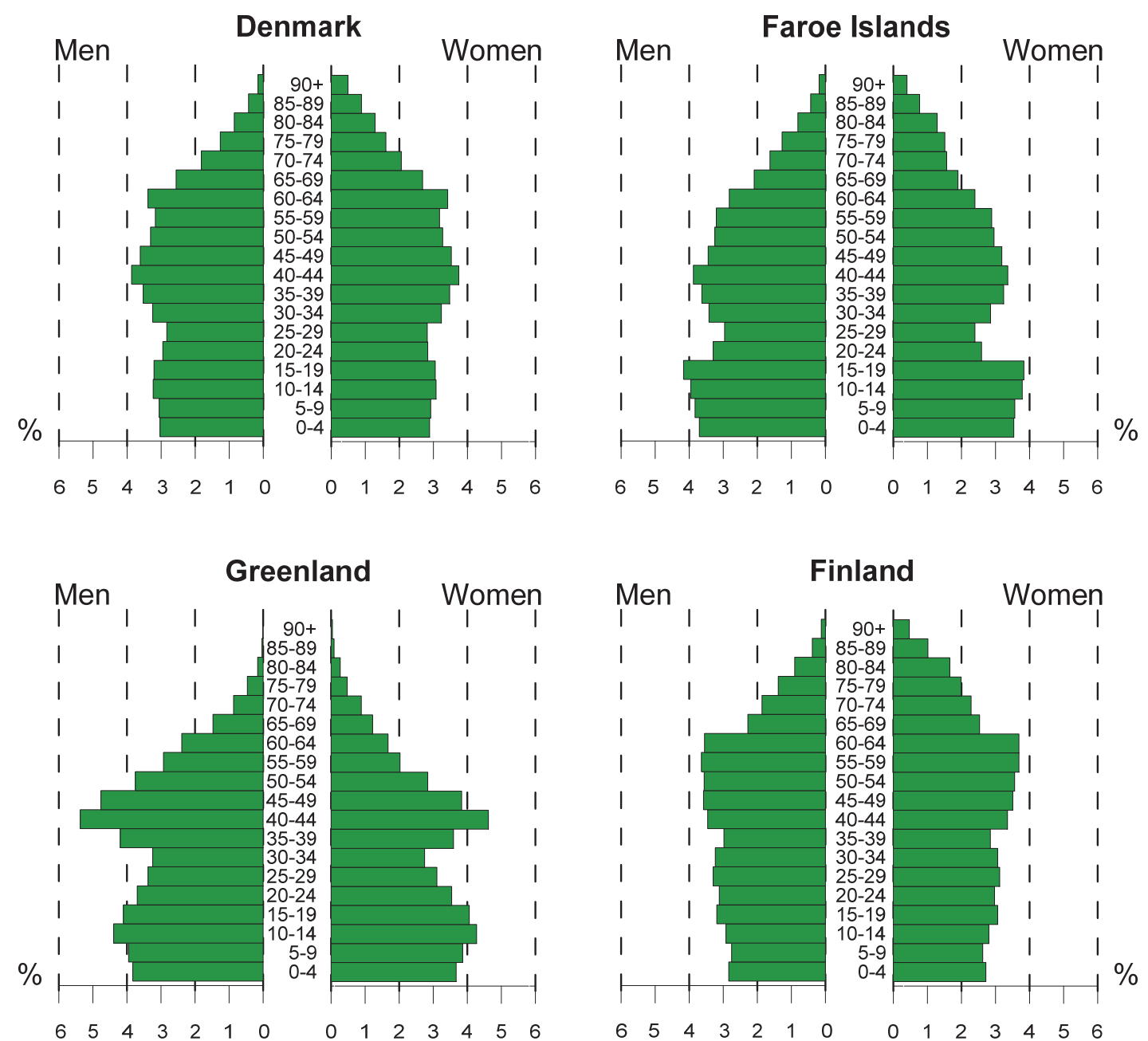

Continues 
Figure 2.1.1 Mean population by gender and age as a percentage of the total population $2015^{1}$, continued
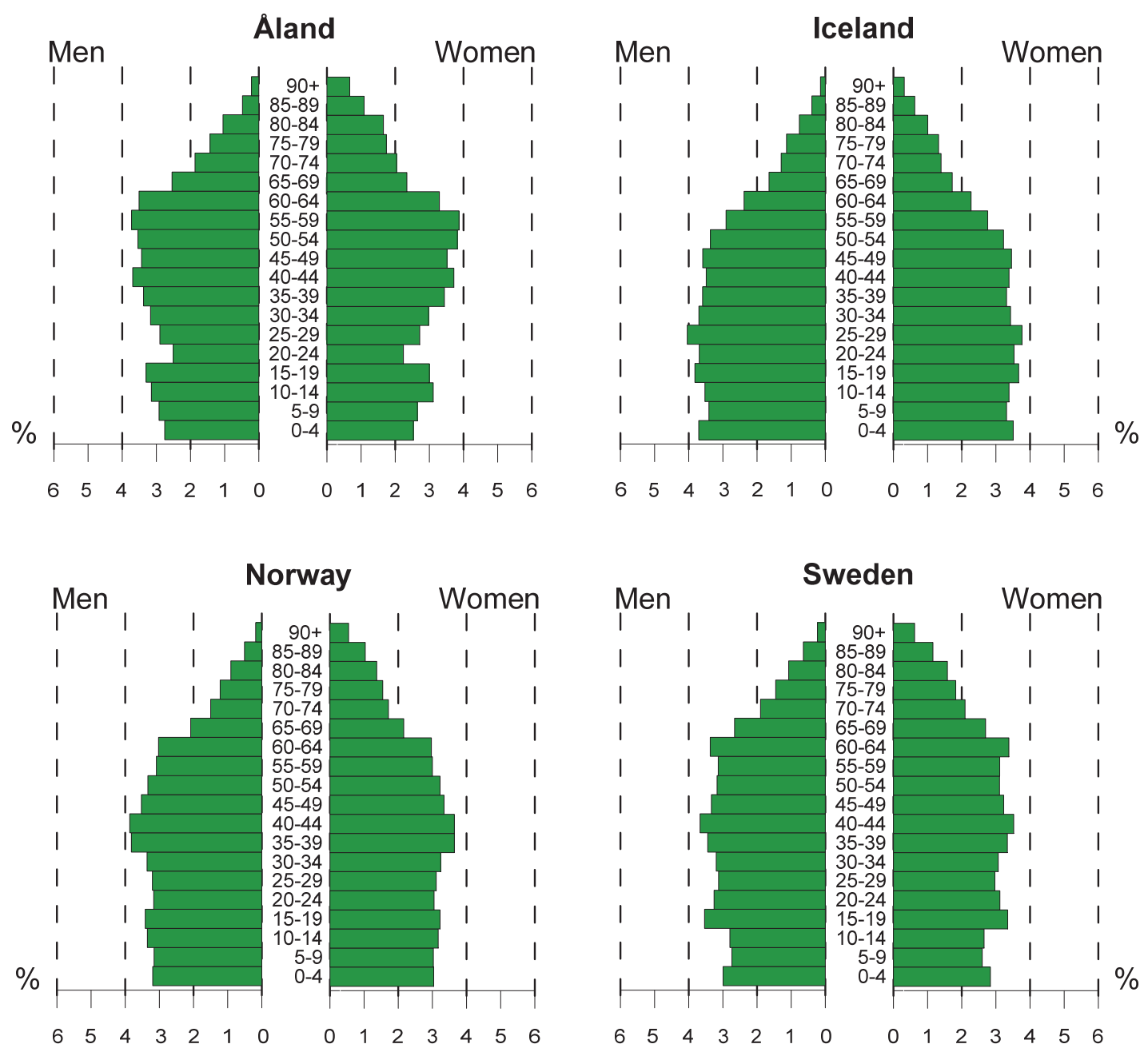

Sweden

Nordic standard population

(Nordic population 2000)

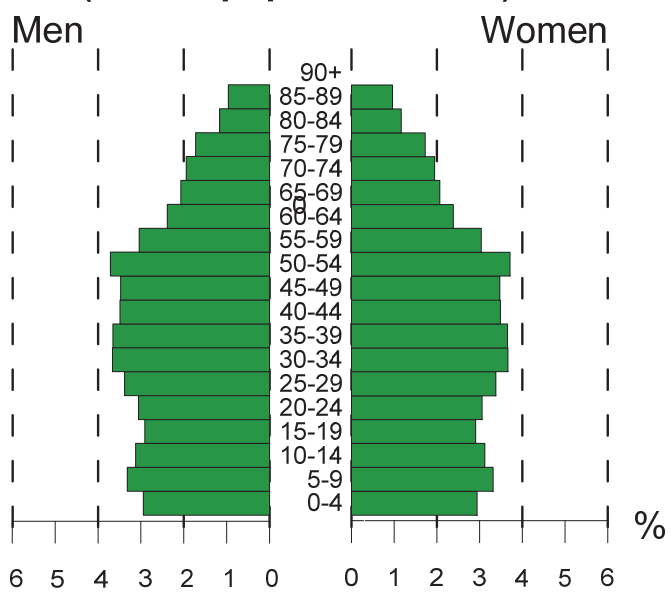

1 Faroe Islands, Greenland, Iceland and Åland: 2011-15 
Table 2.1.1 Mean population 1960-2015

\begin{tabular}{|c|c|c|c|c|c|c|c|c|}
\hline & Denmark & $\begin{array}{l}\text { Faroe } \\
\text { Islands }\end{array}$ & Greenland & Finland & $\begin{array}{l}\text { Of which } \\
\text { Åland }\end{array}$ & Iceland & Norway & Sweden \\
\hline \multicolumn{9}{|l|}{ Men } \\
\hline 1960 & 2265000 & 18000 & .. & 2142263 & 10254 & 89000 & .. & 3740119 \\
\hline 1970 & 2432000 & 20000 & .. & 2219985 & 10249 & 103000 & .. & 4035911 \\
\hline $1980^{1}$ & 2529000 & 22000 & 27000 & 2314843 & 11274 & 115000 & .. & 4035911 \\
\hline 1990 & 2533494 & 24791 & 29853 & 2426204 & 12004 & 127895 & 2097137 & 4228049 \\
\hline 2000 & 2637878 & 23665 & 30002 & 2529341 & 12670 & 140718 & 2224221 & 4386436 \\
\hline 2010 & 2748185 & 25176 & 29939 & 2638416 & 13880 & 159838 & 2443801 & 4669629 \\
\hline 2014 & 2801647 & 25011 & 29742 & 2686114 & 14375 & 163318 & 2583129 & 4843299 \\
\hline 2015 & 2811014 & 25274 & 29634 & 2696677 & 14466 & 166228 & 2611968 & 4901603 \\
\hline \multicolumn{9}{|c|}{ Women } \\
\hline 1960 & 2301000 & 17000 & .. & 2303959 & 10722 & 87000 & .. & 3757848 \\
\hline 1970 & 2474000 & 18000 & .. & 2378351 & 10417 & 101000 & .. & 4045318 \\
\hline $1980^{1}$ & 2593000 & 20000 & 23000 & 2472935 & 11509 & 113000 & $\cdot$ & 4198115 \\
\hline 1990 & 2607445 & 22770 & 25574 & 2572274 & 12414 & 126893 & 2144336 & 4330786 \\
\hline 2000 & 2699466 & 22072 & 26175 & 2651774 & 13072 & 140436 & 2266746 & 4485674 \\
\hline 2010 & 2795634 & 23295 & 26595 & 2736860 & 13991 & 158168 & 2445452 & 4708497 \\
\hline 2014 & 2841829 & 23385 & 26553 & 2775398 & 14417 & 162353 & 2554300 & 4852811 \\
\hline 2015 & 2848701 & 23639 & 26480 & 2782854 & 14484 & 164587 & 2577926 & 4897583 \\
\hline \multicolumn{9}{|c|}{$\begin{array}{l}\text { Men and } \\
\text { Women }\end{array}$} \\
\hline 1960 & 4566000 & 35000 & & 4446222 & 20981 & 176000 & 3591234 & 7497967 \\
\hline 1970 & 4906000 & 39000 & & 4598336 & 20666 & 204000 & 3874133 & 8081229 \\
\hline $1980^{1}$ & 5122000 & 43000 & 50000 & 4787778 & 22783 & 228000 & 4091132 & 8317937 \\
\hline 1990 & 5140939 & 47560 & 55426 & 4998478 & 24418 & 254788 & 4241473 & 8558835 \\
\hline 2000 & 5337344 & 45737 & 56176 & 5181115 & 25741 & 281154 & 4490967 & 8872109 \\
\hline 2010 & 5543819 & 48471 & 56534 & 5375276 & 27871 & 318006 & 4889252 & 9378126 \\
\hline 2014 & 5643475 & 48396 & 56295 & 5461512 & 28791 & 325671 & 5137429 & 9696110 \\
\hline 2015 & 5659715 & 48913 & 56114 & 5479531 & 28950 & 330815 & 5189894 & 9799186 \\
\hline
\end{tabular}

1 The Faroe Islands 1977

Source: DK, Statistics Denmark; FO, Statistics Faroe Islands; GL, Statistics Greenland; FI \& ÅL, Statistics Finland; IS, Statistics Iceland; NO, Statistics Norway; SV, Statistics Sweden 
Table 2.1.2 Mean population, by age groups as a percentage, 1960-2015

\begin{tabular}{|c|c|c|c|c|c|c|c|c|}
\hline & Denmark & $\begin{array}{l}\text { Faroe } \\
\text { Islands }\end{array}$ & Greenland & Finland & Åland & Iceland & Norway & Sweden \\
\hline \multicolumn{9}{|l|}{1960} \\
\hline $0-17$ & 26.3 & 38.4 & .. & 35.3 & 30.2 & 39.9 & 30.6 & 27.3 \\
\hline $18-64$ & 63.2 & 53.6 & .. & 57.4 & 58.6 & 52.1 & 58.5 & 60.9 \\
\hline $65-79$ & .. & .. & .. & 6.4 & 9.4 & .. & .. & 9.9 \\
\hline $80+$ & .. & .. & .. & 0.9 & 1.8 & .. & .. & 1.9 \\
\hline \multicolumn{9}{|l|}{1970} \\
\hline $0-17$ & 31.0 & 36.9 & .. & 30.2 & 26.6 & 38.9 & 29.3 & 24.8 \\
\hline $18-64$ & 56.8 & 54.3 & .. & 60.7 & 60.2 & 52.4 & 57.9 & 61.4 \\
\hline $65-79$ & .. & .. & .. & 8.2 & 11.0 & .. & .. & 11.4 \\
\hline $80+$ & .. & .. & .. & 1.1 & 2.2 & .. & .. & 2.4 \\
\hline \multicolumn{9}{|l|}{$1980^{1}$} \\
\hline $0-17$ & 25.8 & 34.9 & 37.9 & 25.1 & 24.3 & 33.7 & 27.0 & 23.8 \\
\hline $18-64$ & 59.9 & 55.4 & 58.4 & 62.9 & 60.2 & 56.4 & 58.3 & 59.8 \\
\hline $65-79$ & .. & .. & 3.2 & 10.3 & 12.1 & .. & .. & 13.2 \\
\hline $80+$ & .. & .. & 1.1 & 1.8 & 3.5 & .. & .. & 3.2 \\
\hline \multicolumn{9}{|l|}{1990} \\
\hline $0-17$ & 21.3 & 29.5 & 29.6 & 23.0 & 22.0 & 30.0 & 23.3 & 21.9 \\
\hline $18-64$ & 63.1 & 58.7 & 66.6 & 63.6 & 61.5 & 59.4 & 60.4 & 60.4 \\
\hline $65-79$ & 11.9 & 9.5 & 3.3 & 10.6 & 12.5 & 8.1 & 12.6 & 13.5 \\
\hline $80+$ & 3.7 & 2.3 & 0.5 & 2.9 & 4.1 & 2.5 & 3.7 & 4.3 \\
\hline \multicolumn{9}{|l|}{2000} \\
\hline $0-17$ & 21.6 & 27.9 & 31.2 & 21.9 & 22.0 & 27.7 & 23.5 & 21.9 \\
\hline $18-64$ & 63.6 & 58.5 & 63.8 & 63.1 & 61.6 & 60.7 & 61.3 & 60.9 \\
\hline $65-79$ & 10.9 & 10.1 & 4.6 & 11.6 & 11.5 & 8.9 & 10.9 & 12.3 \\
\hline $80+$ & 4.0 & 3.4 & 0.5 & 3.4 & 4.9 & 2.7 & 4.3 & 5.0 \\
\hline \multicolumn{9}{|l|}{2010} \\
\hline $0-17$ & 21.9 & 26.1 & 27.2 & 20.2 & 20.3 & 25.3 & 22.7 & 20.5 \\
\hline $18-64$ & 61.5 & 59.0 & 65.9 & 62.3 & 61.6 & 62.5 & 62.3 & 61.2 \\
\hline $65-79$ & 12.5 & 10.7 & 6.1 & 12.7 & 12.9 & 8.8 & 10.5 & 13.0 \\
\hline $80+$ & 4.1 & 4.1 & 0.8 & 4.8 & 5.2 & 3.4 & 4.5 & 5.3 \\
\hline \multicolumn{9}{|l|}{2014} \\
\hline $0-17$ & 20.8 & 25.5 & 25.5 & 19.7 & 19.8 & 24.6 & 21.9 & 19.8 \\
\hline $18-64$ & 60.8 & 57.9 & 66.9 & 60.6 & 60.1 & 62.2 & 62.1 & 60.1 \\
\hline $65-79$ & 14.2 & 12.1 & 6.6 & 14.6 & 15.0 & 9.6 & 11.7 & 14.7 \\
\hline $80+$ & 4.2 & 4.4 & 0.9 & 5.0 & 5.2 & 3.6 & 4.3 & 5.4 \\
\hline \multicolumn{9}{|l|}{2015} \\
\hline $0-17$ & 20.6 & 25.3 & 25.2 & 19.6 & 19.7 & 24.1 & 21.7 & 20.5 \\
\hline $18-64$ & 60.7 & 57.8 & 67.0 & 60.2 & 59.7 & 62.2 & 62.0 & 59.8 \\
\hline $65-79$ & 14.4 & 12.5 & 6.8 & 15.1 & 15.4 & 10.0 & 12.0 & 14.6 \\
\hline $80+$ & 4.3 & 4.4 & 1.0 & 5.1 & 5.2 & 3.7 & 4.2 & 5.1 \\
\hline
\end{tabular}

1 The Faroe Islands 1977 
Table 2.1.3 Vital statistics per 1000 inhabitants, 2000-2015

\begin{tabular}{|c|c|c|c|c|c|}
\hline & Live births & Deaths & Natural increase & Net migration & $\begin{array}{c}\text { Population } \\
\text { increase }\end{array}$ \\
\hline \multicolumn{6}{|l|}{ Denmark } \\
\hline 2000 & 12.6 & 10.9 & 1.7 & 1.8 & 3.5 \\
\hline 2005 & 11.9 & 10.2 & 1.7 & 1.2 & 2.9 \\
\hline 2010 & 11.5 & 9.8 & 1.6 & 4.0 & 5.7 \\
\hline 2014 & 10.1 & 9.1 & 1.0 & 6.6 & 7.6 \\
\hline 2015 & 10.3 & 9.2 & 1.0 & 8.6 & 9.6 \\
\hline \multicolumn{6}{|c|}{ Faroe Islands } \\
\hline $2006-10$ & 13.4 & 7.9 & 5.5 & -3.7 & 1.8 \\
\hline 2011-15 & 12.6 & 7.9 & 4.8 & -1.0 & 3.8 \\
\hline \multicolumn{6}{|c|}{ Greenland } \\
\hline $2006-10$ & 15.2 & 8.0 & 7.1 & -7.4 & -0.2 \\
\hline 2011-15 & 14.4 & 8.0 & 6.4 & -8.2 & -2.3 \\
\hline \multicolumn{6}{|l|}{ Finland } \\
\hline 2000 & 11.0 & 9.5 & 1.4 & 0.5 & 1.9 \\
\hline 2005 & 11.0 & 9.1 & 1.9 & 1.7 & 3.6 \\
\hline 2010 & 11.4 & 9.5 & 1.9 & 2.6 & 4.4 \\
\hline 2014 & 10.5 & 9.5 & 1.0 & 3.2 & 4.2 \\
\hline 2015 & 10.1 & 9.6 & 0.5 & 2.3 & 2.8 \\
\hline \multicolumn{6}{|l|}{ Åland } \\
\hline $2006-10$ & 10.5 & 9.1 & 1.4 & 5.7 & 7.9 \\
\hline 2011-15 & 9.9 & 9.8 & 0.1 & 5.2 & 6.5 \\
\hline \multicolumn{6}{|l|}{ Iceland } \\
\hline 2000 & 15.3 & 6.5 & 8.8 & 6.1 & 15.3 \\
\hline 2005 & 14.5 & 6.2 & 8.3 & 13.0 & 21.3 \\
\hline 2010 & 15.4 & 6.4 & 9.1 & -6.7 & 2.6 \\
\hline 2014 & 13.4 & 6.3 & 7.1 & 3.4 & 10.5 \\
\hline 2015 & 12.5 & 6.6 & 5.9 & 4.4 & 10.4 \\
\hline \multicolumn{6}{|l|}{ Norway } \\
\hline 2000 & 13.2 & 9.8 & 3.4 & 2.2 & 5.6 \\
\hline 2005 & 12.3 & 8.9 & 3.4 & 4.0 & 7.3 \\
\hline 2010 & 12.6 & 8.5 & 4.1 & 8.7 & 12.7 \\
\hline 2014 & 11.6 & 8.0 & 3.7 & 7.5 & 11.2 \\
\hline 2015 & 11.4 & 7.9 & 3.5 & 5.7 & 9.3 \\
\hline \multicolumn{6}{|l|}{ Sweden } \\
\hline 2000 & 10.2 & 10.5 & -0.3 & 2.8 & 2.4 \\
\hline 2005 & 11.2 & 10.2 & 1.1 & 3.0 & 4.0 \\
\hline 2010 & 12.3 & 9.6 & 2.7 & 5.3 & 8.0 \\
\hline 2014 & 11.9 & 9.2 & 2.7 & 7.8 & 10.6 \\
\hline 2015 & 11.7 & 9.3 & 2.4 & 8.0 & 10.6 \\
\hline
\end{tabular}

Source: DK, Statistics Denmark; FO, Statistics Faroe Islands; GL, Statistics Greenland; FI \& ÅL, Statistics Finland; IS, Statistics Iceland; NO, Statistics Norway; SV, Statistics Sweden 
Table 2.1.4 Average life expectancy, 2000-2015

\begin{tabular}{|c|c|c|c|c|c|c|c|c|c|c|}
\hline \multirow[b]{2}{*}{ Age } & \multicolumn{5}{|c|}{ Men } & \multicolumn{5}{|c|}{ Women } \\
\hline & 0 & 15 & 45 & 65 & 80 & 0 & 15 & 45 & 65 & 80 \\
\hline \multicolumn{11}{|l|}{ Denmark } \\
\hline $2000 / 01$ & 74.5 & 60.1 & 31.6 & 15.2 & 6.8 & 79.2 & 64.7 & 35.5 & 18.2 & 8.5 \\
\hline 2004/05 & 75.6 & 61.2 & 32.5 & 16.0 & 7.0 & 80.2 & 65.7 & 36.4 & 19.0 & 8.8 \\
\hline 2010 & 77.1 & 62.4 & 33.5 & 16.9 & 7.4 & 81.2 & 66.6 & 37.2 & 19.6 & 9.0 \\
\hline 2014 & 78.5 & 63.9 & 34.9 & 17.9 & 7.9 & 82.7 & 68.1 & 38.6 & 20.7 & 9.6 \\
\hline 2015 & 78.6 & 64.0 & 34.9 & 17.9 & 7.9 & 82.5 & 67.9 & 38.4 & 20.5 & 9.5 \\
\hline \multicolumn{11}{|l|}{$\begin{array}{l}\text { Faroe } \\
\text { Islands }\end{array}$} \\
\hline $2006-10$ & 77.2 & 63.1 & 34.2 & 17.0 & 7.2 & 83.0 & 68.6 & 39.1 & 20.8 & 9.5 \\
\hline 2011-15 & 78.8 & 64.5 & 35.6 & 18.3 & 7.8 & 83.8 & 69.5 & 40.0 & 21.6 & 10.1 \\
\hline \multicolumn{11}{|c|}{ Greenland } \\
\hline $2006-10$ & 67.8 & 54.6 & 28.6 & 12.4 & 5.2 & 72.8 & 59.0 & 31.1 & 14.9 & 6.6 \\
\hline 2011-15 & 69.7 & 56.2 & 29.9 & 13.5 & 5.9 & 74.1 & 60.0 & 32.0 & 15.0 & 6.4 \\
\hline \multicolumn{11}{|l|}{ Finland } \\
\hline 2000 & 74.1 & 59.6 & 31.6 & 15.5 & 6.6 & 81.0 & 66.4 & 37.3 & 19.4 & 8.2 \\
\hline 2005 & 75.5 & 61.0 & 32.7 & 16.7 & 7.4 & 82.3 & 67.7 & 38.6 & 20.7 & 9.1 \\
\hline 2010 & 76.7 & 62.0 & 33.7 & 17.3 & 7.6 & 83.2 & 68.5 & 39.2 & 21.2 & 9.4 \\
\hline 2014 & 78.2 & 63.5 & 34.9 & 18.0 & 8.0 & 83.9 & 69.1 & 39.8 & 21.5 & 9.7 \\
\hline 2015 & 78.4 & 63.7 & 35.0 & 18.0 & 8.1 & 84.1 & 69.3 & 39.9 & 21.6 & 9.7 \\
\hline \multicolumn{11}{|l|}{ Åland } \\
\hline $2004-08$ & 79.0 & 64.3 & 35.3 & 17.5 & 7.8 & 83.1 & 68.8 & 39.3 & 21.0 & 9.6 \\
\hline $2009-13$ & 79.2 & 64.2 & 35.1 & 17.9 & 8.1 & 84.2 & 69.5 & 40.0 & 21.2 & 10.0 \\
\hline \multicolumn{11}{|l|}{ Iceland } \\
\hline 2000 & 77.6 & 63.1 & 34.4 & 17.3 & 7.5 & 81.4 & 66.7 & 37.1 & 19.5 & 8.4 \\
\hline 2005 & 79.2 & 64.5 & 35.6 & 18.0 & 7.7 & 83.1 & 68.4 & 39.0 & 20.7 & 9.4 \\
\hline 2010 & 79.5 & 64.8 & 36.0 & 18.2 & 7.7 & 83.5 & 68.8 & 39.3 & 20.8 & 9.4 \\
\hline 2014 & 80.6 & 65.9 & 36.9 & 19.0 & 8.2 & 83.6 & 69.1 & 39.7 & 21.3 & 9.8 \\
\hline 2015 & 81.0 & 66.3 & 37.4 & 19.4 & 8.4 & 83.6 & 69.1 & 39.7 & 21.3 & 9.7 \\
\hline \multicolumn{11}{|l|}{ Norway } \\
\hline 2000 & 76.0 & 61.5 & 33.2 & 16.1 & 6.8 & 81.4 & 66.8 & 37.6 & 19.7 & 8.6 \\
\hline 2005 & 77.7 & 63.2 & 34.5 & 17.1 & 7.3 & 82.5 & 67.9 & 38.6 & 20.6 & 9.3 \\
\hline 2010 & 78.9 & 64.2 & 35.4 & 17.9 & 7.8 & 83.2 & 68.5 & 39.1 & 21.0 & 9.6 \\
\hline 2014 & 80.0 & 65.4 & 36.4 & 18.7 & 8.2 & 84.1 & 69.3 & 39.8 & 21.5 & 9.9 \\
\hline 2015 & 80.4 & 65.7 & 36.6 & 18.8 & 8.2 & 84.1 & 69.4 & 39.9 & 21.5 & 9.9 \\
\hline \multicolumn{11}{|l|}{ Sweden } \\
\hline 2000 & 77.4 & 62.8 & 34.0 & 16.7 & 7.1 & 82.0 & 67.4 & 38.0 & 20.1 & 8.9 \\
\hline 2005 & 78.4 & 63.8 & 34.9 & 17.4 & 7.5 & 82.8 & 68.1 & 38.7 & 20.6 & 9.3 \\
\hline 2010 & 79.5 & 64.8 & 35.8 & 18.2 & 7.9 & 83.5 & 68.8 & 39.3 & 21.1 & 9.6 \\
\hline 2014 & 80.4 & 65.6 & 36.7 & 18.9 & 8.2 & 84.1 & 69.3 & 39.8 & 21.5 & 9.8 \\
\hline 2015 & 80.3 & 65.6 & 36.7 & 18.9 & 8.2 & 84.0 & 69.3 & 39.8 & 24.1 & 9.7 \\
\hline
\end{tabular}


Figure 2.1.2 Live births and natural increase per 1000 inhabitants, 2000-2015
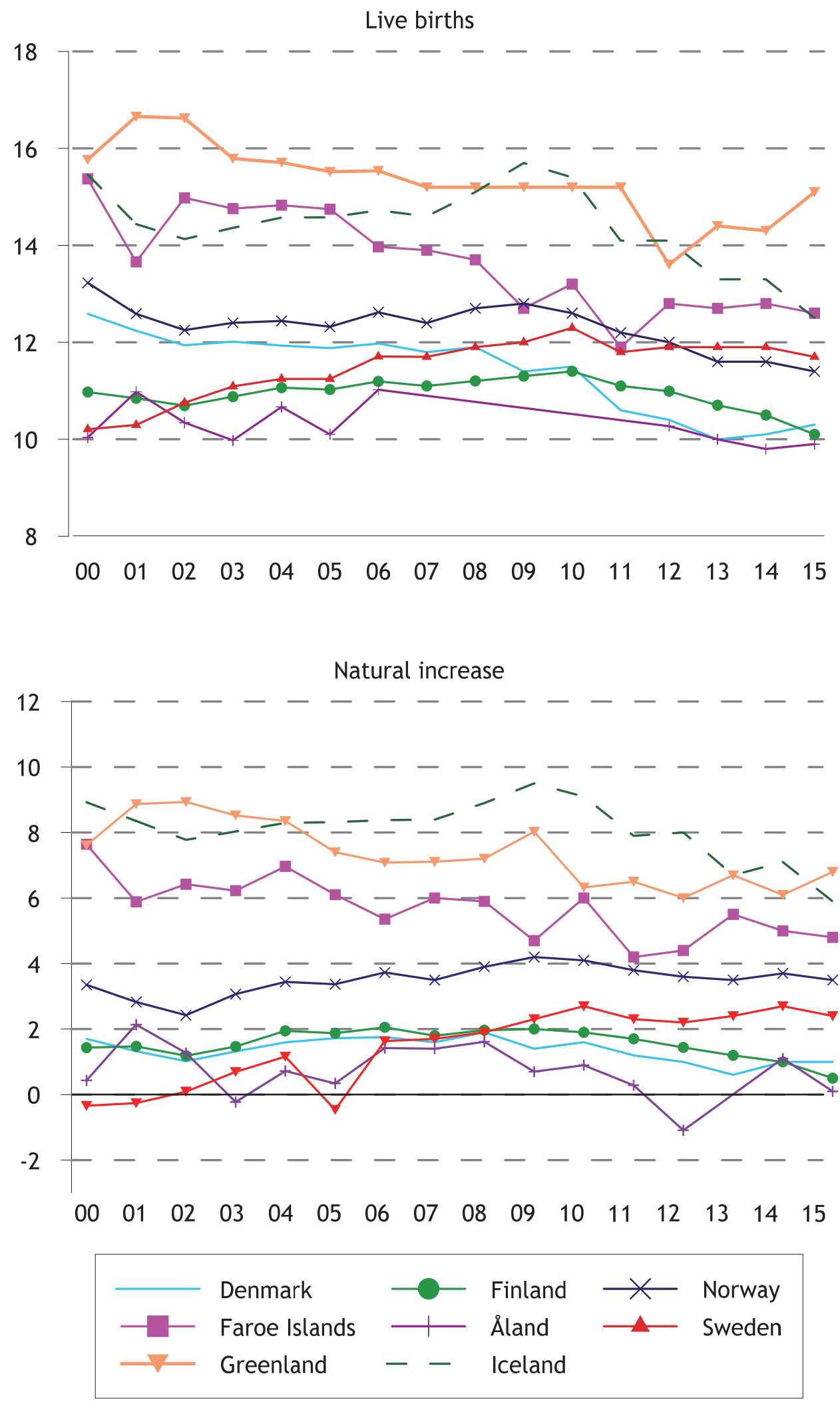
Figure 2.1.3 Deaths per 1000 inhabitants, 2000-2015

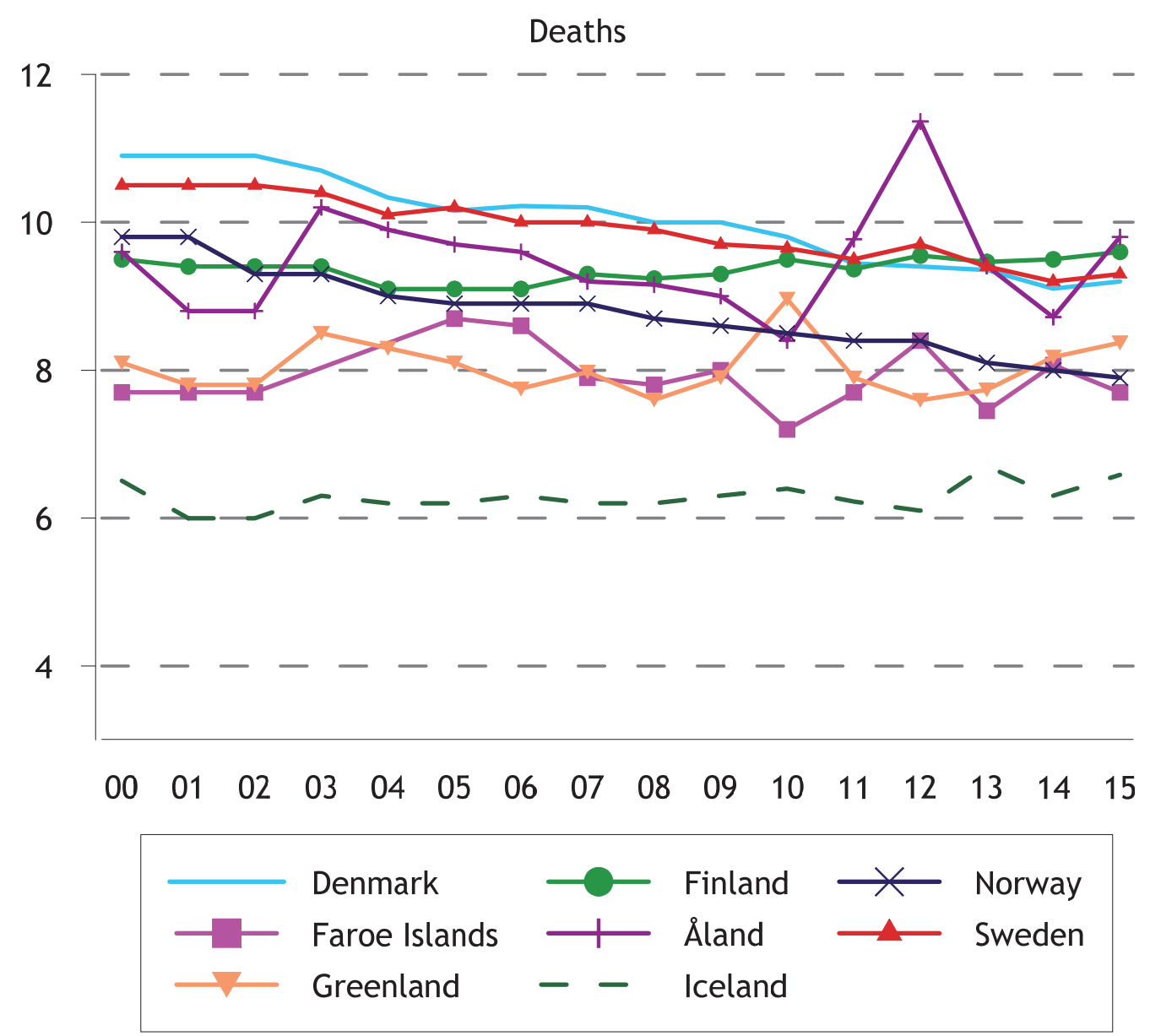


Figure 2.1.4 Life expectancy at birth 1900, 1950 and 2015
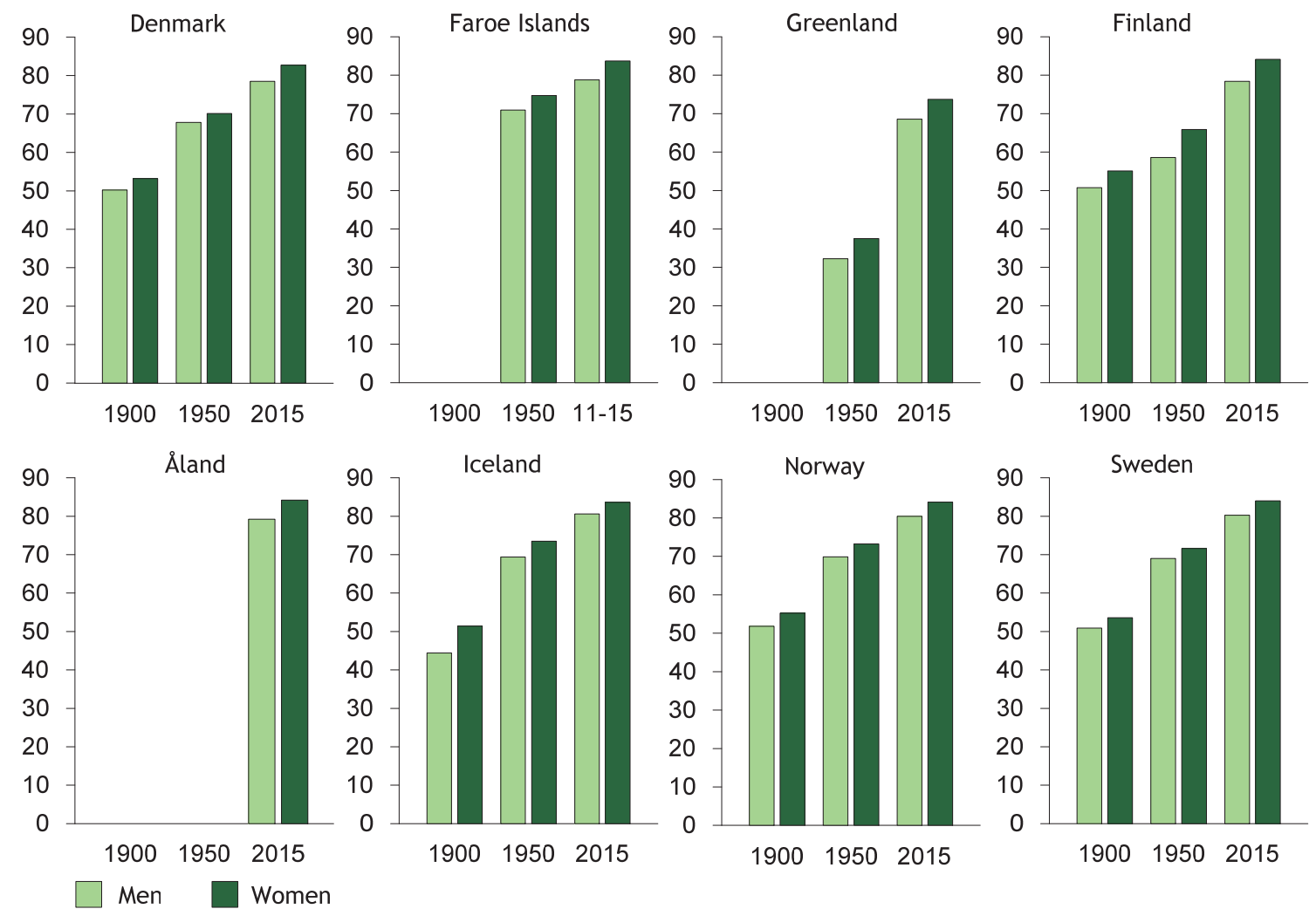


\subsection{Fertility, births, infant mortality and contraception}

In recent years, the overall development in fertility has resulted in Finland now having the lowest fertility rate in the Nordic countries, while the rates remain high in the Faroe Islands, Greenland and Iceland, particularly for the youngest age groups.

In all the Nordic countries, it is possible to obtain treatment for infertility, paid for by the public health services (in Iceland and Norway, however, there is a higher user charge for in vitro fertilization (IVF) treatment than for other types of treatment). As shown in Table 2.2.2, more and more people receive such treatment, and a significant proportion of live births is the result of IVF. A large number of births resulting from IVF are still multiple births. The rate of multiple births among spontaneous pregnancies (non-IVF-pregnancies) are 1.0-1.5 percent.

Internationally, the Nordic countries are characterized by having very low perinatal mortality. Greenland has the highest perinatal mortality rate among the Nordic countries. The other countries lie relatively close to each other. Changes in perinatal mortality during this period are the result of changes in the definition of gestational ages. The time limit for spontaneous abortion and stillbirth is 22 weeks in all the Nordic countries except for the Faroe Islands and Greenland, where the limit is 28 weeks.

Greenland also has the highest, and Åland, Finland and Iceland have the lowest mortality rate for the first year of life.

The sale of hormonal contraceptives varies substantially among the Nordic countries, but these differences have become smaller over time.

There are no comparable Nordic statistics on the use of coils and condoms.

Use of emergency contraception is relatively widespread in the Nordic countries. Use is highest in Norway and lowest in the Faroe Islands, Denmark and Greenland.

Since the middle of the 1970s, induced abortion has been available in most of the Nordic countries. In Sweden, it is a requirement that the abortion takes place before the end of the 18th week of gestation, while in the other Nordic countries it must be performed before the end of the 12th week of gestation. However, induced abortion may also be carried out after the 12th or 18th week of gestation, but only following special assessment and permission.

In Denmark, Greenland, Norway and Sweden, it is solely up to the pregnant woman herself to decide whether an abortion is to be performed, while permission is required in the Faroe Islands, Finland, Åland and Iceland. Such permission is given on the basis of social and/or medical criteria.

Abortion rates vary greatly in the Nordic countries. 
Table 2.2.1 Live births and fertility rates, 2000-2015

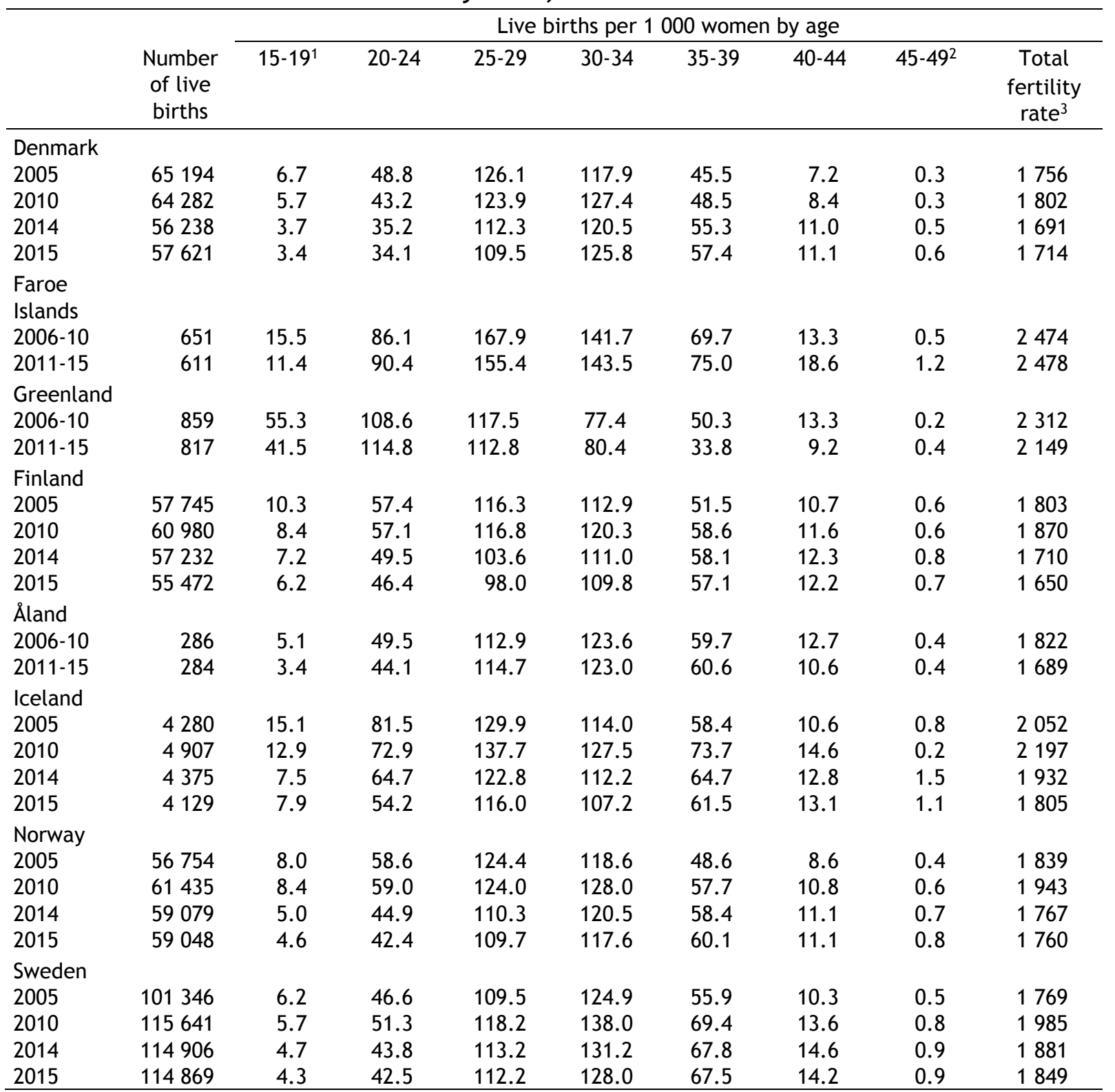

1 Births by women under 15 years are included

2 Births by women over 50 years are included

3 Total fertility rate: The imputed number of live births experienced by women during their fertile period, assuming that their mortality is zero during this period and that the age-specific fertility rates for the year in question are valid throughout the reproductive period

Source: DK, Statistics Denmark; FO, Statistics Faroe Islands; GL, Statistics Greenland; FI \& ÅL, Statistics Finland; IS, Statistics Iceland; NO, Statistics Norway; SV, Statistics Sweden 
Table 2.2.2 Assisted reproduction technologies 2000-2014

\begin{tabular}{|c|c|c|c|c|c|}
\hline & Denmark & Finland & Iceland & Norway & Sweden \\
\hline \multicolumn{6}{|c|}{ Treatments, IVF+ICSI } \\
\hline 2000 & 7077 & 4323 & 298 & 4029 & 6586 \\
\hline 2005 & 7222 & 4731 & 462 & 5067 & 8062 \\
\hline 2010 & 11721 & 4861 & 618 & 6557 & 9593 \\
\hline 2013 & 11212 & 4561 & 476 & 6387 & 9111 \\
\hline 2014 & 11339 & 4548 & 409 & .. & .. \\
\hline \multicolumn{6}{|c|}{$\begin{array}{l}\text { Frozen embryo transfers, } \\
\text { FET }\end{array}$} \\
\hline 2000 & 792 & 2488 & 83 & 301 & 1208 \\
\hline 2005 & 1500 & 2960 & 161 & 1698 & 3458 \\
\hline 2010 & 2275 & 3280 & 257 & 2046 & 4948 \\
\hline 2013 & 2721 & 3274 & 316 & 3112 & 5264 \\
\hline 2014 & 2712 & 3384 & 296 & .. & .. \\
\hline \multicolumn{6}{|c|}{$\begin{array}{l}\text { Number of live births, } \\
\text { IVF+ ICSI + FET }\end{array}$} \\
\hline 2000 & 1678 & 1382 & 147 & 1097 & 2237 \\
\hline 2005 & 1786 & 1534 & 167 & 1521 & 2874 \\
\hline 2010 & 2123 & 1858 & 192 & 1885 & 3882 \\
\hline $2013^{2}$ & 2553 & 1686 & 135 & 2005 & 3939 \\
\hline 2014 & 2978 & 1658 & 150 & .. & .. \\
\hline
\end{tabular}

IVF = In vitro fertilization

ICSI = Intracytoplasmic sperm injection

FET = Frozen embryo transfer

1 Based on the year of treatment, not on the year of birth

2 Denmark, calculated on the basis of expected number of births and expected number of children born

Source: DK, the Danish Health Data Authority; FI, THL; IS, Art Medica; NO, the Norwegian Directorate of Health; SV, National Board of Health and Welfare 
Table 2.2.3 Assisted reproduction technologies, treatments per 1000 women aged 15-49 years, 2000-20141

\begin{tabular}{|c|c|c|c|c|c|}
\hline & Denmark & Finland & Iceland & Norway & Sweden \\
\hline \multicolumn{6}{|c|}{$I V F+I C S I$} \\
\hline 2000 & & 3.5 & & & 3.3 \\
\hline 2005 & 5.8 & 3.9 & 6.3 & 4.7 & 4.0 \\
\hline 2010 & 9.3 & 4.2 & 8.0 & 5.8 & 4.6 \\
\hline 2013 & 8.9 & 4.0 & 6.2 & 5.4 & 4.3 \\
\hline 2014 & 9.0 & 4.0 & 5.3 & 5.4 & .. \\
\hline \multicolumn{6}{|l|}{ FET } \\
\hline 2000 & & 2.0 & & & 0.6 \\
\hline 2005 & 1.5 & 2.5 & 2.2 & 1.0 & 1.3 \\
\hline 2010 & 1.8 & 2.8 & 3.3 & 1.8 & 2.1 \\
\hline 2013 & 2.2 & 2.8 & 4.1 & 1.9 & 2.5 \\
\hline 2014 & 2.2 & 2.9 & 3.8 & 2.6 & .. \\
\hline \multicolumn{6}{|c|}{ Total IVF + ICSI, FET } \\
\hline 2000 & .. & 5.6 & .. & .. & 3.9 \\
\hline 2005 & 7.8 & 6.6 & 8.5 & 5.7 & 5.3 \\
\hline 2010 & 11.1 & 7.0 & 11.3 & 7.6 & 6.7 \\
\hline $2013^{2}$ & 11.1 & 6.8 & 10.3 & 7.3 & 6.7 \\
\hline 2014 & 11.1 & 6.9 & 9.1 & 8.0 & .. \\
\hline \multicolumn{6}{|c|}{$\begin{array}{l}\text { Multiple births, per cent } \\
\text { of all births after IVF' }\end{array}$} \\
\hline 2000 & & 17.3 & & & \\
\hline 2005 & 20.3 & 11.3 & 28.5 & 24.2 & 6.5 \\
\hline 2010 & 15.5 & 9.6 & 8.3 & 11.0 & 5.2 \\
\hline $2013^{2}$ & 13.6 & 6.5 & 7.4 & 11.1 & 4.7 \\
\hline 2014 & 10.2 & 4.8 & 8.6 & 11.1 & .. \\
\hline \multicolumn{6}{|c|}{$\begin{array}{l}\text { Children born in multiple } \\
\text { births, per cent of all } \\
\text { children born after IVF }\end{array}$} \\
\hline 2000 & & 29.7 & . & & \\
\hline 2005 & 34.1 & 20.5 & 44.3 & 39.2 & 12.2 \\
\hline 2010 & 13.4 & 17.7 & 19.8 & & .. \\
\hline $2013^{2}$ & 24.0 & 12.0 & 14.8 & 20.0 & .. \\
\hline 2014 & 18.8 & 9.0 & 15.9 & 20.0 & .. \\
\hline \multicolumn{6}{|c|}{$\begin{array}{l}\text { IVF, ICSI and FET per cent } \\
\text { of all live births }{ }^{2}\end{array}$} \\
\hline 2000 & & 2.5 & & & \\
\hline 2005 & 3.3 & 2.6 & 3.9 & 2.6 & 2.7 \\
\hline 2010 & 3.3 & 3.1 & 3.9 & 3.1 & 12.2 \\
\hline $2013^{2}$ & 5.1 & 3.0 & 3.1 & 3.0 & 12.8 \\
\hline 2014 & 5.1 & 2.9 & 3.5 & 3.4 & ". \\
\hline
\end{tabular}

IVF = In vitro fertilization

ICSI = Intracytoplasmic sperm injection

FET = Frozen embryo transfer

1 Based on the year of treatment, not on the year of birth

2 Denmark, calculated on the basis of expected number of births and expected number of children born

Source: DK, the Danish Health Data Authority; FI, THL; IS, Art Medica; NO, the Norwegian Directorate of Health; SV, National Board of Health and Welfare 
Table 2.2.4 Stillbirths and infant mortality ${ }^{1}, 2000-2014$

\begin{tabular}{|c|c|c|c|c|c|c|c|c|}
\hline & \multicolumn{2}{|c|}{ Number } & \multicolumn{2}{|c|}{ Per 1000 births } & \multicolumn{4}{|c|}{ Deaths per 1000 live births } \\
\hline & $\begin{array}{l}\text { Still- } \\
\text { births }\end{array}$ & $\begin{array}{l}\text { Infant } \\
\text { deaths }\end{array}$ & $\begin{array}{l}\text { Still- } \\
\text { births }\end{array}$ & $\begin{array}{c}\text { Perinatal } \\
\text { deaths }^{2}\end{array}$ & $\begin{array}{c}\text { First } 24 \\
\text { hours }\end{array}$ & 1-6 days & $\begin{array}{l}\text { 7-27 } \\
\text { days }\end{array}$ & $\begin{array}{l}\text { Total } \\
\text { under } \\
1 \text { year }\end{array}$ \\
\hline \multicolumn{9}{|l|}{ Denmark } \\
\hline 2005 & 302 & 303 & 4.7 & 7.9 & 2.1 & 1.1 & 0.6 & 4.7 \\
\hline 2010 & 255 & 216 & 4.0 & 6.2 & 1.5 & 0.6 & 0.4 & 3.4 \\
\hline 2013 & 230 & 186 & 4.1 & 5.1 & 1.5 & 0.8 & 0.4 & 3.3 \\
\hline 2014 & 293 & 137 & 5.1 & 6.1 & 2.3 & 1.0 & 0.4 & 4.3 \\
\hline \multicolumn{9}{|l|}{$\begin{array}{l}\text { Faroe } \\
\text { Islands }\end{array}$} \\
\hline 2005-09 & 2 & 3 & 3.6 & 6.0 & 1.2 & 1.2 & 0.3 & 4.8 \\
\hline $2010-14$ & 1 & 4 & 1.9 & 4.4 & 2.2 & 0.3 & 1.0 & 4.8 \\
\hline \multicolumn{9}{|c|}{ Greenland ${ }^{3}$} \\
\hline 2005-09 & 5 & 15 & 5.3 & 9.2 & 3.7 & 0.2 & 1.4 & 10.0 \\
\hline $2010-14$ & 4 & 15 & 4.9 & 10.0 & 5.2 & - & 1.2 & 8.9 \\
\hline \multicolumn{9}{|l|}{ Finland } \\
\hline 2005 & 182 & 174 & 3.1 & 4.9 & 1.0 & 0.7 & 0.3 & 3.0 \\
\hline 2010 & 181 & 140 & 3.0 & 4.1 & 0.6 & 0.5 & 0.4 & 2.3 \\
\hline 2013 & 149 & 102 & 2.6 & 3.4 & 0.5 & 0.4 & 0.2 & 1.9 \\
\hline 2014 & 163 & 124 & 2.8 & 3.9 & 0.6 & 0.5 & 0.4 & 2.2 \\
\hline \multicolumn{9}{|l|}{ Åland } \\
\hline 2005-09 & 1 & 1 & 0.7 & 1.4 & 0.7 & - & - & 0.7 \\
\hline $2010-14$ & 2 & 1 & 1.4 & 2.1 & 0.7 & - & - & 0.7 \\
\hline \multicolumn{9}{|l|}{ Iceland ${ }^{3}$} \\
\hline 2005 & 8 & 10 & 1.9 & 3.3 & 0.7 & 0.7 & 0.2 & 2.3 \\
\hline 2010 & 9 & 11 & 1.8 & 2.9 & 0.8 & 0.2 & 0.2 & 2.2 \\
\hline 2013 & 4 & 8 & 0.9 & 1.6 & 0.5 & 0.2 & 0.7 & 1.8 \\
\hline 2014 & 11 & 9 & 2.5 & 3.4 & 0.7 & 0.2 & 0.7 & 2.1 \\
\hline \multicolumn{9}{|l|}{ Norway } \\
\hline 2005 & 230 & 171 & 4.0 & 5.5 & 1.0 & 0.5 & 0.5 & 3.0 \\
\hline 2010 & 246 & 157 & 3.9 & 5.1 & 0.8 & 0.3 & 0.5 & 2.5 \\
\hline 2013 & 227 & 142 & 3.8 & 4.9 & 0.8 & 0.4 & 0.5 & 2.4 \\
\hline 2014 & 262 & 144 & 4.4 & 5.8 & 1.0 & 0.5 & 0.4 & 2.4 \\
\hline \multicolumn{9}{|l|}{ Sweden ${ }^{4}$} \\
\hline 2005 & 301 & 246 & 3.0 & 4.1 & 0.5 & 0.6 & 0.4 & 2.4 \\
\hline 2010 & 426 & 294 & 3.7 & 4.8 & 0.5 & 0.6 & 0.4 & 2.5 \\
\hline 2013 & 441 & 306 & 3.9 & 5.2 & 0.7 & 0.7 & 0.3 & 2.7 \\
\hline 2014 & 456 & 251 & 4.0 & 5.1 & 0.6 & 0.6 & 0.3 & 2.2 \\
\hline
\end{tabular}

1 Calculated according to year of death

2 Stillbirths and deaths in the first week of life

3 A child is considered stillborn at the $28^{\text {th }}$ week of pregnancy or later

4 As of 1st July 2008, a child is considered stillborn at the $22^{\text {nd }}$ week of pregnancy or later

Source: DK, the Danish Health Data Authority; FO, Chief Medical Officer in the Faroe Islands; GL, Chief Medical Officer; FI \& ÅL, Statistics Finland; IS, Statistics Iceland; NO, Statistics Norway; SV, Statistics Sweden 
Figure 2.2.1 Total fertility rate ${ }^{1}$, 2000-2015

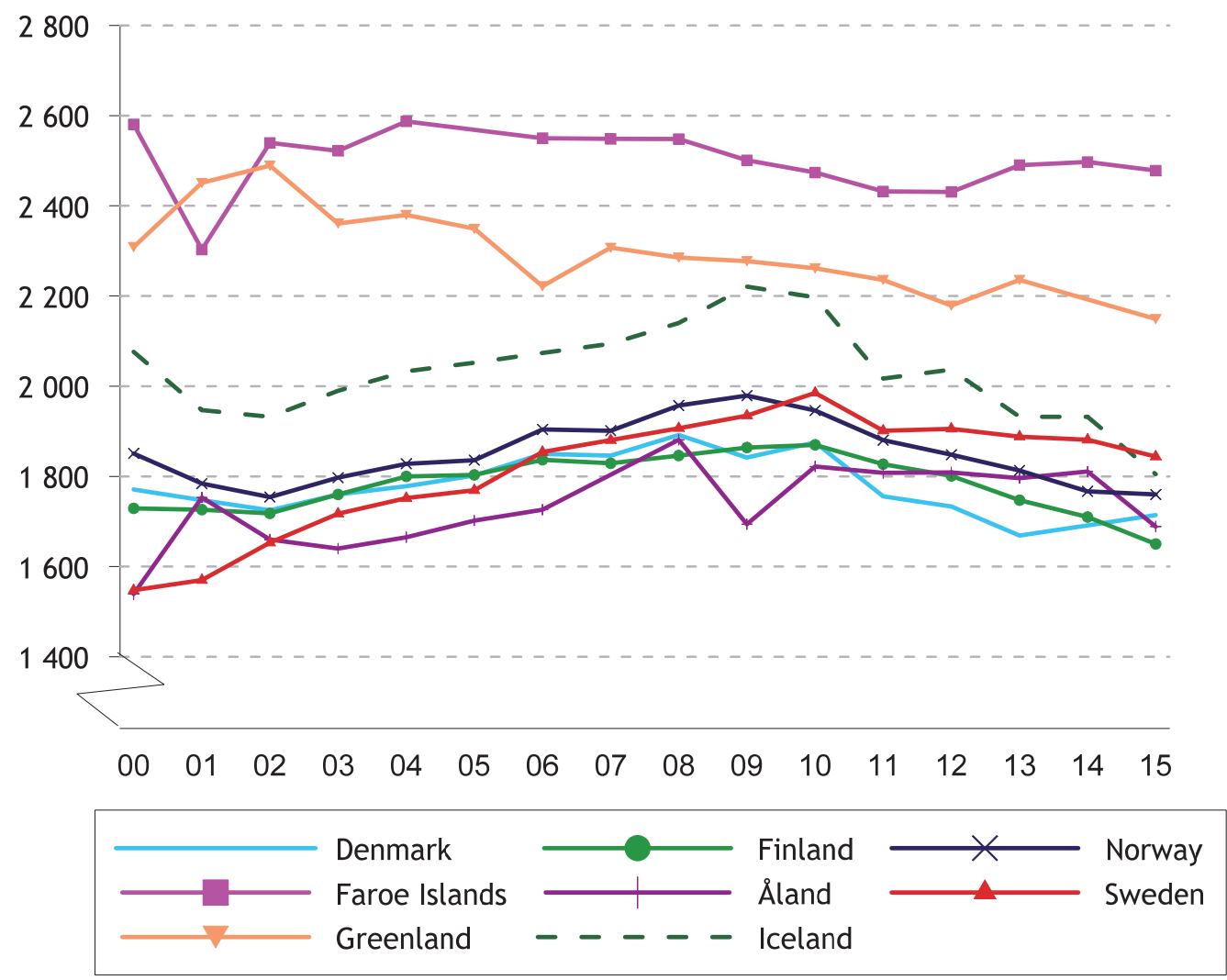

1 Total fertility rate: The imputed number of live births experienced by women during their fertile period, assuming that their mortality is zero during this period and that the age-specific fertility rates for the year in question are valid throughout the reproductive period 
Figure 2.2.2 Assisted reproduction technologies, percentages of all live births 2000-2014

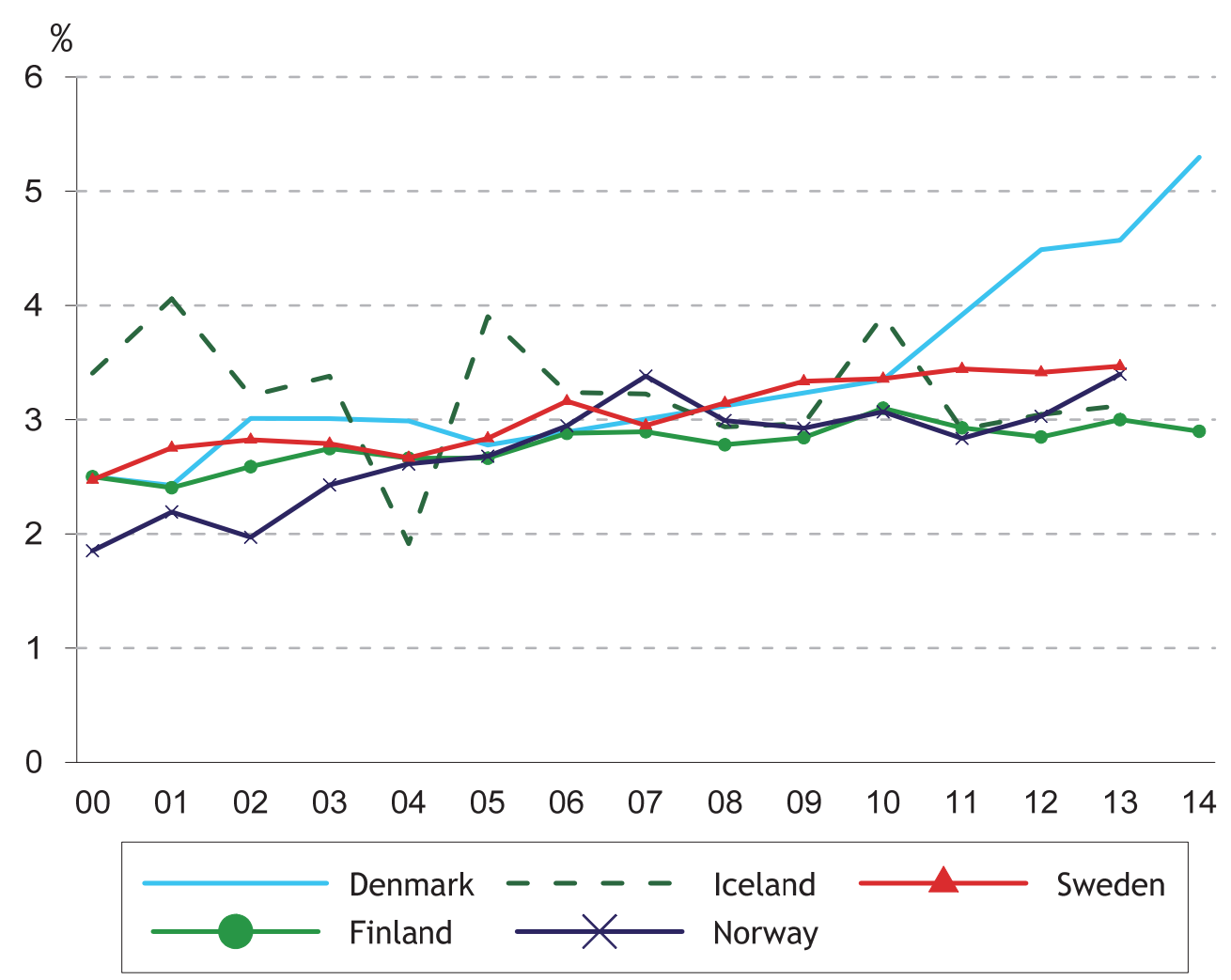


Figure 2.2.3 Infant deaths, and perinatal ${ }^{1}$ deaths per 1000 births, 2000-2014 Infant mortality per 1000 births

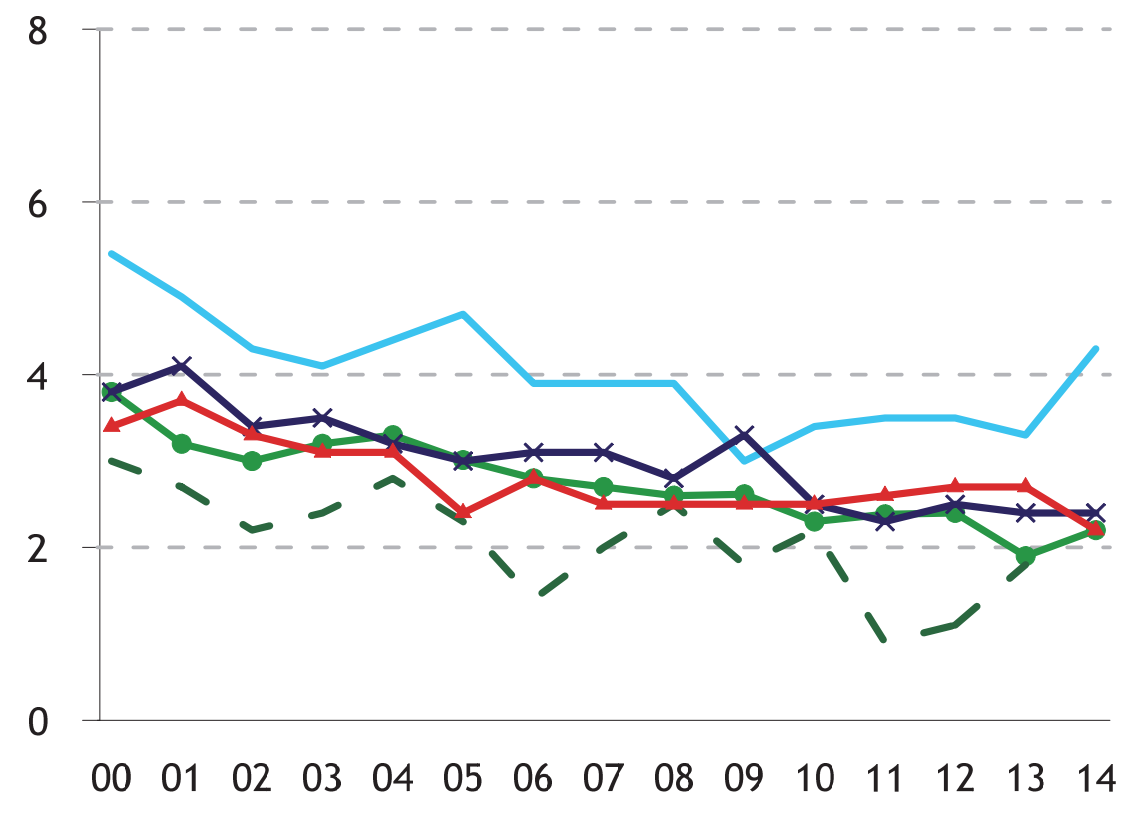

Perinatal deaths per 1000 births

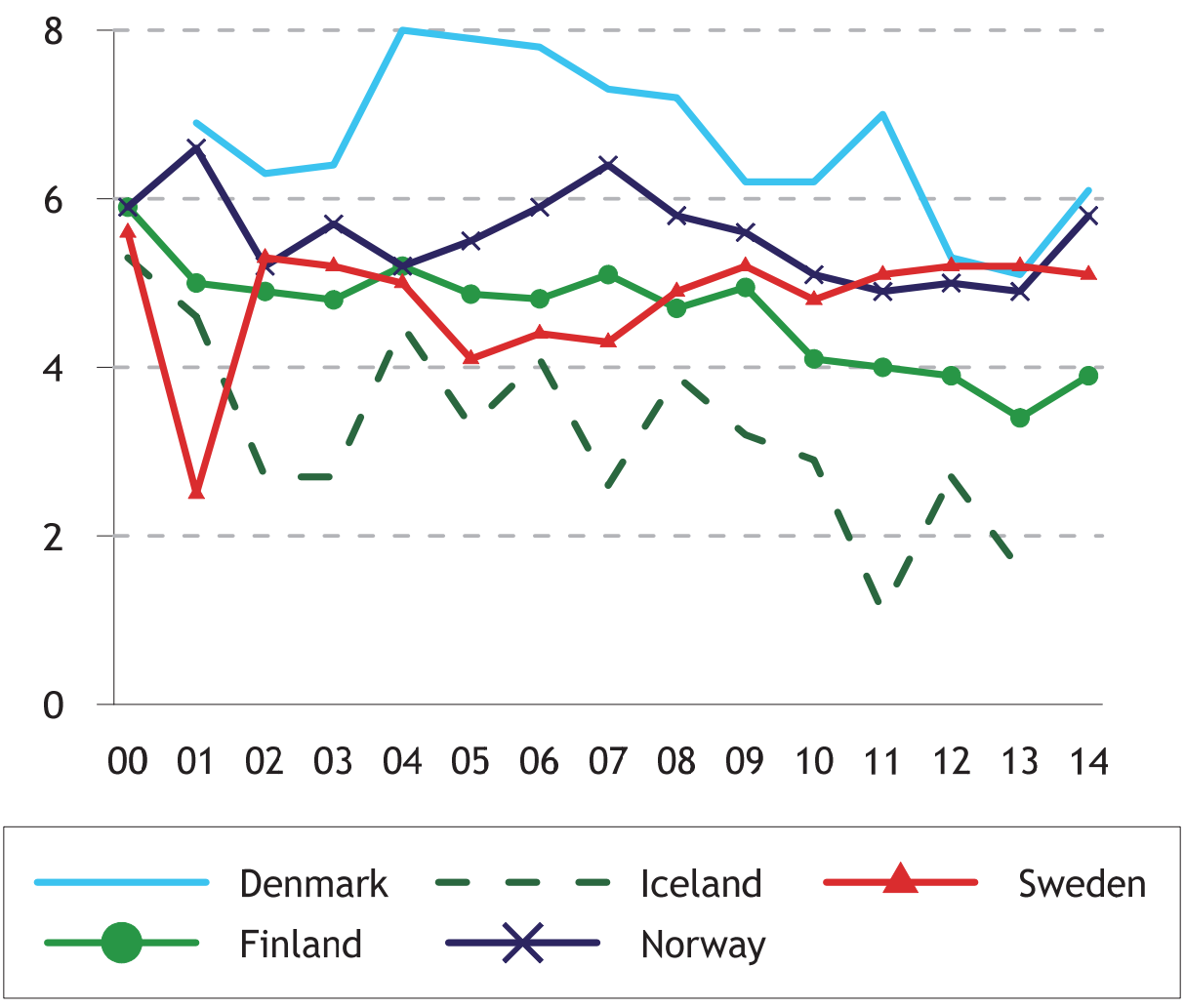

1 Perinatal deaths are the total number of stillbirths and deaths in the first week of life 
Table 2.2.5 Stillbirths and deaths during the first year of life per 1000 births, with a birth weight of 1000 grams or more, total and per 1000 births, 2000-20141

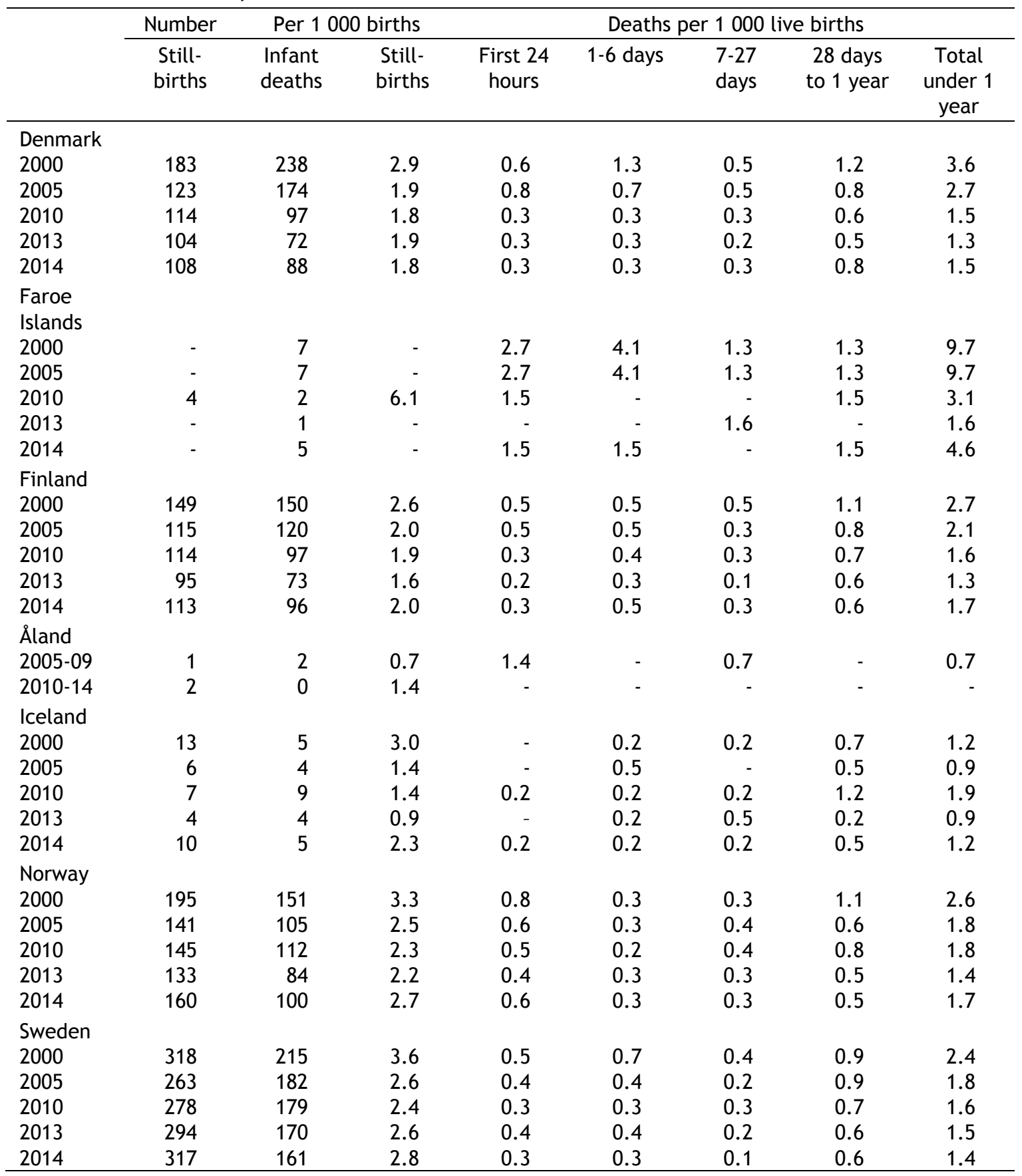

1 Calculated according to year of birth

Source: DK, the Danish Health Data Authority; FI, Statistics Finland \& THL; IS, Medical Birth Registry of Iceland \& Statistics Iceland; NO, National Institute of Public Health, (MFR); SV, the National Board of Health and Welfare 
Table 2.2.6 Sales of hormonal contraceptives. ATC code G03A ${ }^{1}$ and G02BB. DDD per 1000 women aged 15-49 years/day, 2005-2015

\begin{tabular}{lcccccccc}
\hline & Denmark & $\begin{array}{c}\text { Faroe } \\
\text { Islands }\end{array}$ & Greenland & Finland & Åland & Iceland & Norway & Sweden \\
\hline 2005 & 294 & 238 & 314 & 190 & 190 & 192 & 201 & 213 \\
2010 & 287 & 232 & 302 & 201 & 167 & 204 & 217 & 207 \\
2012 & 278 & 208 & 285 & 204 & 184 & 208 & 222 & 202 \\
2014 & 272 & 196 & 254 & 209 & 204 & 210 & 222 & 191 \\
2015 & 265 & 197 & 250 & 209 & 202 & 212 & 236 & 183 \\
\hline
\end{tabular}

1 Excl. Implants (G03AC08), injections (G03AC06) and emergency contraceptives (G03AD). Intrauterine contraceptives (G02BA) are not included

Source: DK, the Danish Health Data Authority; FO, Chief Pharmaceutical Officer; GL, National Pharmacy; FI \& ÅL, Finnish Medicines Agency; IS, Icelandic Medicines Agency; NO, Norwegian Institute of Public Health; SV, Swedish eHealth Agency

Table 2.2.7 Emergency contraceptives ATC code G03AD: number of packages sold per 1000 women aged 15-49 years, 2005-2015

\begin{tabular}{lcccccccr}
\hline & Denmark & $\begin{array}{c}\text { Faroe } \\
\text { Islands }\end{array}$ & Greenland & Finland & Åland & Iceland & Norway & Sweden \\
\hline 2005 & 63 & 52 & 41 & 78 & 79 & 87 & 119 & 83 \\
2010 & 81 & 81 & 53 & 83 & 84 & 91 & 140 & 100 \\
2012 & 80 & 77 & 88 & 77 & 81 & 84 & 135 & 106 \\
2014 & 79 & 78 & 69 & 87 & 85 & 75 & 121 & 105 \\
2015 & 81 & 75 & 63 & 87 & 88 & 83 & 119 & 115 \\
\hline
\end{tabular}

Source: DK, the Danish Health Data Authority; FO, Chief Pharmaceutical Officer; GL, National Pharmacy; FI \& ÅL, Finnish Medicines Agency; IS, Icelandic Medicines Agency; NO, Norwegian Institute of Public Health; SV, Swedish eHealth Agency 
Table 2.2.8 Number of induced abortions, 2000-2014

\begin{tabular}{|c|c|c|c|c|c|c|c|c|c|c|}
\hline \multirow{4}{*}{$\begin{array}{l} \\
\text { Denmark } \\
\text { 2000-04 }\end{array}$} & \multirow[b]{2}{*}{$\begin{array}{l}\text { Number of } \\
\text { abortions }\end{array}$} & \multicolumn{7}{|c|}{ Abortions per 1000 women aged } & \multirow[b]{2}{*}{$\begin{array}{c}\text { Total } \\
\text { abortion } \\
\text { rate }^{3}\end{array}$} & \multirow[b]{2}{*}{$\begin{array}{c}\text { Abortions } \\
\text { per } 1000 \\
\text { live } \\
\text { births }\end{array}$} \\
\hline & & $15-19^{1}$ & \multirow[t]{2}{*}{$20-24$} & \multirow[t]{2}{*}{$25-29$} & \multirow[t]{2}{*}{$30-34$} & \multirow[t]{2}{*}{$35-39$} & \multirow[t]{2}{*}{$40-44$} & $45-49^{2}$ & & \\
\hline & & & & & & & & & & \\
\hline & 15365 & 14.5 & 20.4 & 17.7 & 17.0 & 13.0 & 4.8 & 0.4 & 439 & 237 \\
\hline 2005 & 15295 & 16.0 & 21.3 & 17.4 & 16.6 & 13.1 & 5.2 & 0.5 & 450 & 238 \\
\hline 2010 & 15227 & 16.4 & 22.0 & 18.0 & 15.8 & 12.3 & 5.5 & 0.5 & 452 & 233 \\
\hline 2013 & 15834 & 13.2 & 24.8 & 18.5 & 15.4 & 13.2 & 5.8 & 0.6 & 458 & 270 \\
\hline 2014 & 15097 & 11.9 & 23.3 & 19.3 & 15.1 & 11.9 & 5.6 & 0.5 & 438 & 265 \\
\hline \multicolumn{11}{|c|}{ Faroe Islands } \\
\hline 2005-09 & 41 & 3.5 & 7.8 & 3.5 & 7.1 & 4.5 & 2.9 & 0.2 & 151 & 62 \\
\hline $2010-14$ & 31 & 3.1 & 6.7 & 4.4 & 5.0 & 4.8 & 2.2 & 0.6 & 134 & 50 \\
\hline \multicolumn{11}{|c|}{ Greenland } \\
\hline 2005-09 & 869 & 69.5 & 138.0 & 97.5 & 59.4 & 26.6 & 7.9 & 0.8 & 2206 & 1008 \\
\hline $2010-14$ & 823 & 59.1 & 122.4 & 91.0 & 53.7 & 31.0 & 9.6 & 0.3 & 2013 & 1004 \\
\hline \multicolumn{11}{|l|}{ Finland } \\
\hline $2000-04$ & 10869 & 15.3 & 16.4 & 12.6 & 10.7 & 7.7 & 3.1 & 0.2 & 330 & 192 \\
\hline 2005 & 10972 & 15.0 & 18.2 & 12.8 & 10.4 & 7.9 & 3.4 & 0.2 & 338 & 190 \\
\hline 2010 & 10243 & 12.1 & 17.0 & 13.1 & 9.8 & 7.7 & 3.0 & 0.2 & 315 & 167 \\
\hline 2013 & 10130 & 10.5 & 17.5 & 12.7 & 9.9 & 7.7 & 3.1 & 0.3 & 309 & 174 \\
\hline 2014 & 9779 & 9.2 & 16.8 & 12.9 & 9.9 & 7.3 & 3.2 & 0.2 & 298 & 170 \\
\hline \multicolumn{11}{|l|}{ Åland } \\
\hline 2005-09 & 66 & 15.3 & 27.0 & 21.8 & 11.4 & 8.3 & 3.9 & 0.2 & 440 & 238 \\
\hline $2010-14$ & 68 & 12.8 & 31.2 & 20.0 & 14.5 & 7.6 & 2.8 & 0.0 & 444 & 241 \\
\hline \multicolumn{11}{|l|}{ Iceland } \\
\hline 2000-04 & 940 & 21.4 & 23.4 & 17.3 & 13.6 & 9.2 & 4.6 & 0.3 & 449 & 225 \\
\hline 2005 & 868 & 15.6 & 23.9 & 18.2 & 12.3 & 8.0 & 4.1 & 0.2 & 412 & 210 \\
\hline 2010 & 978 & 16.0 & 23.0 & 19.2 & 13.4 & 11.4 & 3.5 & 0.5 & 435 & 199 \\
\hline 2013 & 966 & 13.1 & 24.6 & 19.1 & 13.7 & 10.3 & 4.8 & 0.3 & 430 & 223 \\
\hline 2014 & 951 & 12.7 & 24.8 & 19.9 & 12.9 & 10.5 & 2.7 & 0.1 & 418 & 217 \\
\hline \multicolumn{11}{|l|}{ Norway } \\
\hline 2000-04 & 14008 & 17.3 & 27.1 & 19.4 & 15.1 & 10.6 & 3.8 & 0.3 & 470 & 246 \\
\hline 2005 & 13991 & 15.4 & 27.4 & 20.5 & 15.1 & 11.0 & 4.0 & 0.3 & 468 & 247 \\
\hline 2010 & 15738 & 14.1 & 29.2 & 23.1 & 16.9 & 11.7 & 4.4 & 0.4 & 500 & 256 \\
\hline 2013 & 14748 & 10.1 & 24.5 & 21.8 & 16.9 & 11.1 & 4.5 & 0.3 & 446 & 250 \\
\hline 2014 & 14061 & 9.6 & 21.8 & 20.7 & 15.9 & 11.4 & 4.2 & 0.4 & 420 & 238 \\
\hline \multicolumn{11}{|l|}{ Sweden } \\
\hline $2000-04$ & 33009 & 22.6 & 29.4 & 23.3 & 19.8 & 15.2 & 6.3 & 0.6 & 586 & 345 \\
\hline 2005 & 34978 & 23.4 & 31.4 & 24.3 & 19.8 & 16.0 & 7.8 & 0.7 & 617 & 345 \\
\hline 2010 & 37696 & 20.3 & 33.3 & 26.7 & 21.5 & 16.3 & 7.1 & 0.8 & 620 & 330 \\
\hline $2013^{4}$ & 36600 & .. & .. & .. &.. & .. & .. & .. & .. & 325 \\
\hline $2014^{5}$ & 36629 & 15.1 & 29.6 & 27.3 & 22.3 & 16.7 & \multicolumn{2}{|c|}{7.9} & 595 & 319 \\
\hline & & & rs are in & & & & & & & \\
\hline 2 Aborti & women 50 & ears or $n$ & nore are & ncluded & & & & & & \\
\hline $\begin{array}{l}3 \text { The to } \\
\text { calcul }\end{array}$ & $\begin{array}{l}\text { ortion rate is } \\
\text { om the age } s\end{array}$ & $\begin{array}{l}\text { the num } \\
\text { pecific a }\end{array}$ & $\begin{array}{l}\text { ber of al } \\
\text { bortion }\end{array}$ & $\begin{array}{l}\text { ortions } \\
\text { ates for }\end{array}$ & $\begin{array}{l}\text { r } 1000 \\
\text { e curr }\end{array}$ & $\begin{array}{l}\text { romen } \\
\text { t peric }\end{array}$ & xpectec & to live tc & be 50 yea & \\
\hline $\begin{array}{l}4 \text { Due to } \\
\text { halted }\end{array}$ & $\begin{array}{l}\text { grns over the } \\
\text { g in spring } 20\end{array}$ & $\begin{array}{l}\text { level of } \\
13\end{array}$ & personal & detail re & corded, & egistrati & of of sta & tistics on & abortions & was \\
\hline $\begin{array}{l}5 \text { New m } \\
30-34 \text {, }\end{array}$ & for data coll 40 or more & ection $\mathrm{fr}$ & om 2014 & Among & ther ch & nges: $s$ & age grc & ups $\leq 19$ & $20-24,25-2$ & 9 \\
\hline Source: & ional aborti & on regist & & & & & & & & \\
\hline
\end{tabular}


Figure 2.2.4 Sales of emergency contraceptives per 1000 women aged 15-49 years, 2005-2015

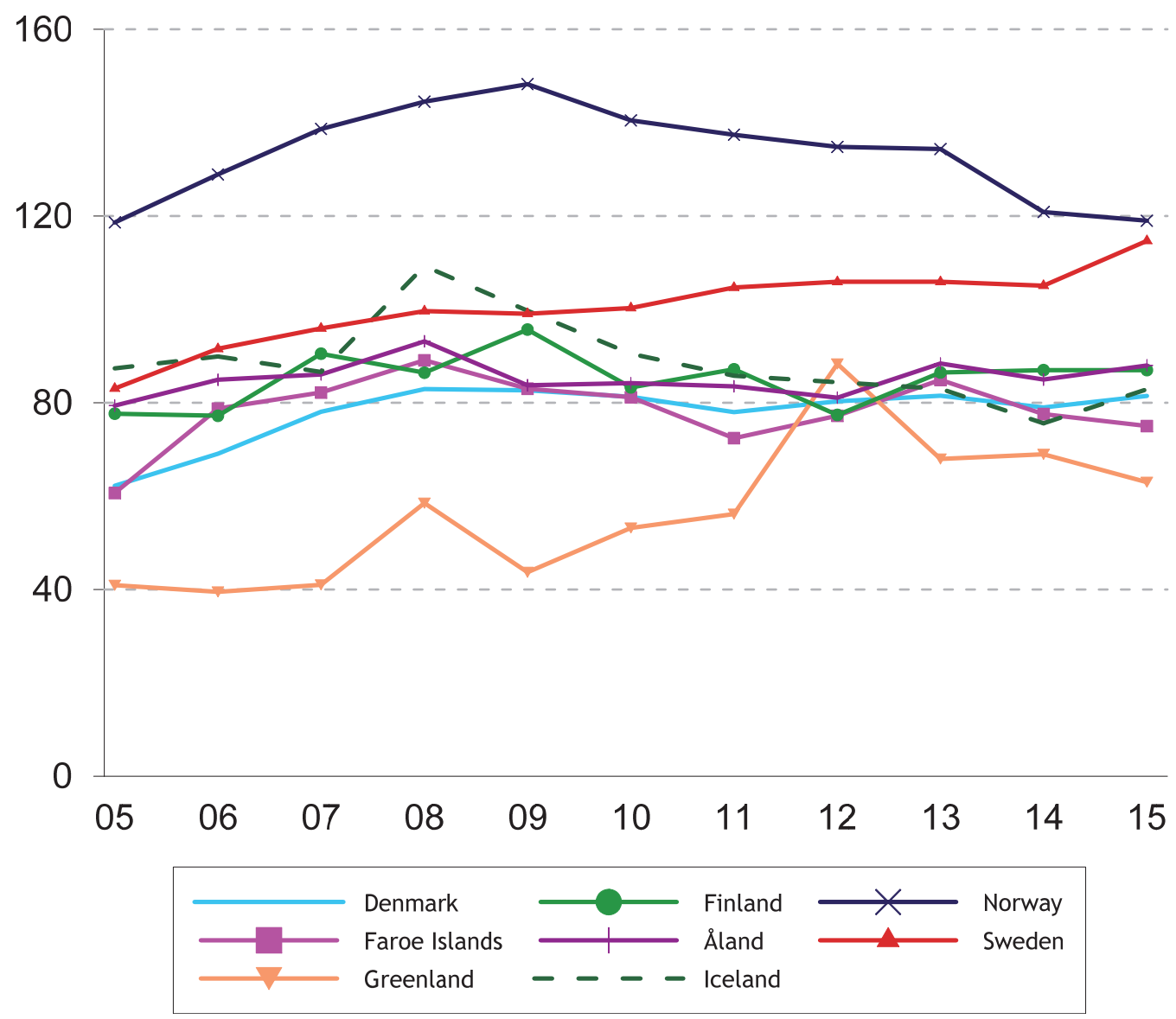

Source: DK, The Danish Health Data Authority; FO, Chief Pharmaceutical Officer; GL, National Pharmacy; FI \& ÅL, FIMEA; IS, Icelandic Medicines Agency; NO, Norwegian Institute of Public Health; SV, Swedish eHealth Agency 
Figure 2.2.5 Total abortion rate, 2000-2014

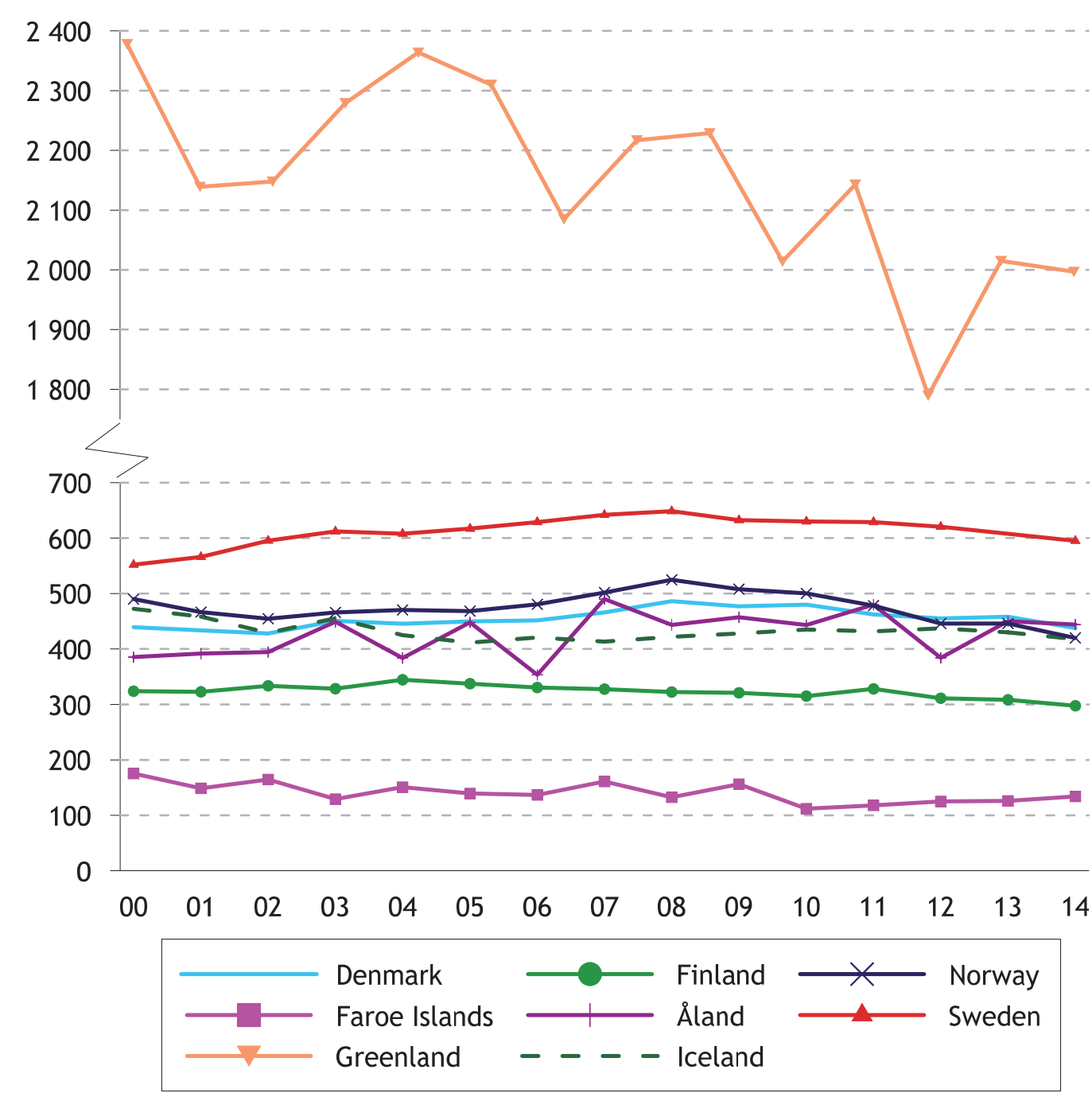

Source: The national abortion registers 
Population and Fertility 


\section{Chapter 3}

\section{Morbidity, Medical Treatment, Accidents and Pharmaceutical Products}

\section{Extra material}

Background tables Nowbase.org/Publications

The Nordic Cancer Union

\section{Introduction}

This chapter begins with a description of a number of diseases that can be related to lifestyle and social behaviour, followed by data on the incidence of cancer. This is followed by a presentation of treatment provided outside hospitals and in hospitals according to diagnostic group and in connection with major surgical procedures. Following this, data on accident occurrences and discharges from hospitals due to accidents are presented. Finally data on consumption of pharmaceutical products are presented. 


\subsection{Diseases related to lifestyle}

This section deals with a number of diseases that can be related to the lifestyle and social behaviour of people in the population and that can be treated either outside hospitals or in hospitals.

Although the number of smokers in the Nordic countries has been decreasing during recent years, there continues to be large differences in the number of smokers, both for men and for women and some differences between countries. Among other things, this pattern of behaviour is reflected in the incidence of lung cancer, as shown in Figure 3.1.1, in which the rates reflect behaviour several years previously.

The proportion of people who are overweight is an increasing problem in the Nordic countries. The proportion is highest in Greenland and Iceland, and lowest in Faroe Islands, Sweden and Norway.

With regard to alcohol consumption, the statistics are inadequate, as the available data are based on sales figures. These figures indicate that the largest consumption/sales are in Denmark and Finland, followed by Greenland, whereas consumption/sales in the other countries is at about the same level. Accordingly, the number of treatment periods/discharges from hospital for alcoholic liver diseases is highest in Denmark and Finland.

This publication previously included data on the occurrence of hepatitis B and C, but as the information from the different countries is not comparable, this table has been left out.

The number of diagnosed cases of tuberculosis is relatively stable in the Nordic Countries.

The incidence of HIV infection is relatively stable, with the highest incidence in Denmark and Norway. The trend is related to the new methods of treatment that result in infected people having a longer period with HIV infection, and therefore a longer period of time before AIDS breaks out. This gives a greater number of potential carriers with the risk of infecting other people. In comparison, Figure 4.1.5 shows that mortality as a result of HIV/AIDS has been at a stable low level in all countries since the end of the 1990s.

Without doubt, chlamydia infection is the most common sexually transmitted disease in the Nordic countries. It is also the most common cause of infertility among women. There are some differences between the countries, but Greenland is radically different. The disease is often without symptoms, and is therefore probably underreported.

A marked fall in the incidence of the traditional sexually transmitted infections, gonorrhoea and syphilis, has been seen in all countries over the past 20 years. However, there are certain notable exceptions, with Greenland being radically different from the other countries. 
Table 3.1.1 Self-reported obesity rate, population aged 15+, 2014

\begin{tabular}{lccccccc}
\hline & Denmark & $\begin{array}{c}\text { Faroe } \\
\text { Islands }\end{array}$ & $\begin{array}{c}\text { Green- } \\
\text { land }\end{array}$ & Finland & Iceland ${ }^{2,3}$ & Norway $^{4}$ & Sweden $^{5}$ \\
\hline & 2013 & 2014 & 2014 & 2014 & 2012 & 2015 & 2014 \\
\hline $\begin{array}{l}\text { Proportion of people } \\
\text { with BMI } \geq 30 \text {, men }\end{array}$ & 14 & 10 & 26 & 17 & 17 & 13 & 12 \\
$\begin{array}{l}\text { Proportion of people } \\
\text { with BMI } \geq 30 \text {, wom- }\end{array}$ & 14 & 13 & 29 & 15 & 16 & 12 & 12 \\
en & & & & & & & \\
\hline
\end{tabular}

1 Measured data from the Population survey in Greenland, 2014. Age 18+

2 Age 15-64

2 Self-reported data from the survey: "Health and Wellbeing of Icelanders 2012". Age 18-79

3 Age $16+$

4 Age $16-84$

Source: DK, the National Boards of Health; FO, Public Health Council; IS, Directorate of Health. Selfreported data from the survey Health and Wellbeing of Icelanders 2012;

FI, THL; Health Behaviour and Health among the Finnish Adult Population; SV, Statistics Sweden

Table 3.1.2 Percentage of daily smokers by gender 2014

\begin{tabular}{lcccccc}
\hline & Denmark & $\begin{array}{c}\text { Faroe } \\
\text { Islands }\end{array}$ & Finland & Iceland & Norway & Sweden \\
\hline \multicolumn{1}{c}{ Age } & $16+$ & $15+$ & $15-64$ & $15+$ & $16-74$ & $16-84$ \\
\hline $\begin{array}{l}\text { Smoking men as \% of men } \\
\text { in the age group }\end{array}$ & 16 & 20 & 17 & 14 & 13 & 12 \\
$\begin{array}{l}\text { Smoking women as \% of } \\
\text { women in the age group }\end{array}$ & 17 & 24 & 14 & 14 & 13 & 12 \\
\hline
\end{tabular}

Source: DK, the National Board of Health; FO, Public Health Council; FI, THL; Health Behaviour and Health among the Finnish Adult Population; IS, Directorate of Health (from regular surveys on tobacco consumption); NO, Norwegian Directorate of Health; SV, Statistics Sweden

Table 3.1.3 Percentage of users of snuff by gender 2014

\begin{tabular}{|c|c|c|c|c|c|c|}
\hline & Denmark & $\begin{array}{l}\text { Faroe } \\
\text { Islands }^{1}\end{array}$ & Finland & Iceland $^{2}$ & Norway $^{3}$ & Sweden \\
\hline Age & $16+$ & $15+$ & $15-64$ & $15-85$ & $16-74$ & $16+$ \\
\hline $\begin{array}{l}\text { Men using snuff as \% of } \\
\text { men in the age group }\end{array}$ & $<1$ & . & 6 & 7 & 15 & 20 \\
\hline $\begin{array}{l}\text { Women using snuff as \% of } \\
\text { women in the age group }\end{array}$ & $<1$ & . & - & 3 & 4 & 3 \\
\hline
\end{tabular}

1 A survey from the Council of Public Health from March 2015 showed, that 38\% of pupils in 9th grade have tried using snuff, of which $15 \%$ have tried it within the previous 30 days. The survey does not cover all of the population and was not subdivided by sex

2 Self-reported data from a telephone and web survey. Regular oral use of snuff

3 Daily use

Source: DK, the National Board of Health; FO, Public Health Council; FI, THL; Health Behavior and Health among the Finnish Adult Population; IS, Directorate of Health (from regular surveys on tobacco consumption); NO, Statistics Norway; SV, Statistics Sweden 
Figure 3.1.1 Rates for new cases of lung cancer per 1000000 inhabitants

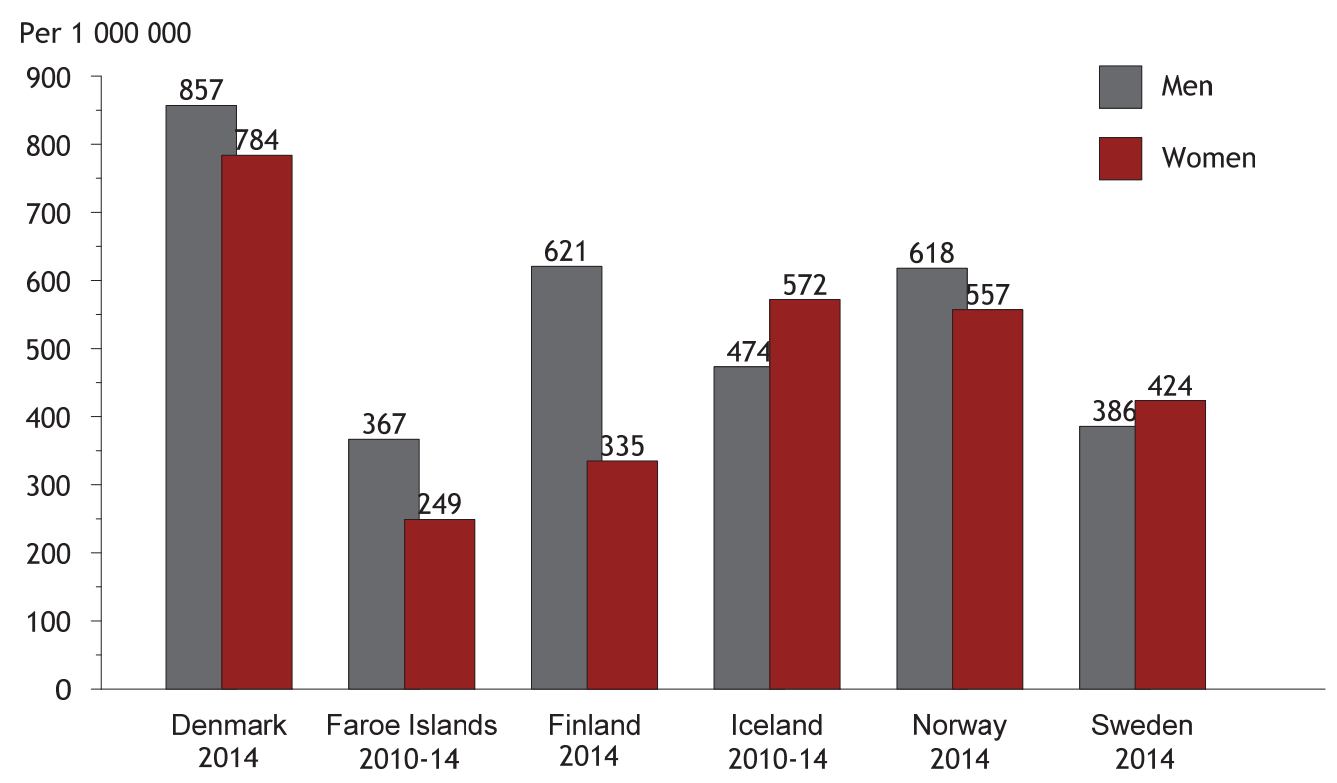


Figure 3.1.2 New cases of lung cancer per 1000000 inhabitants, 2000-2014
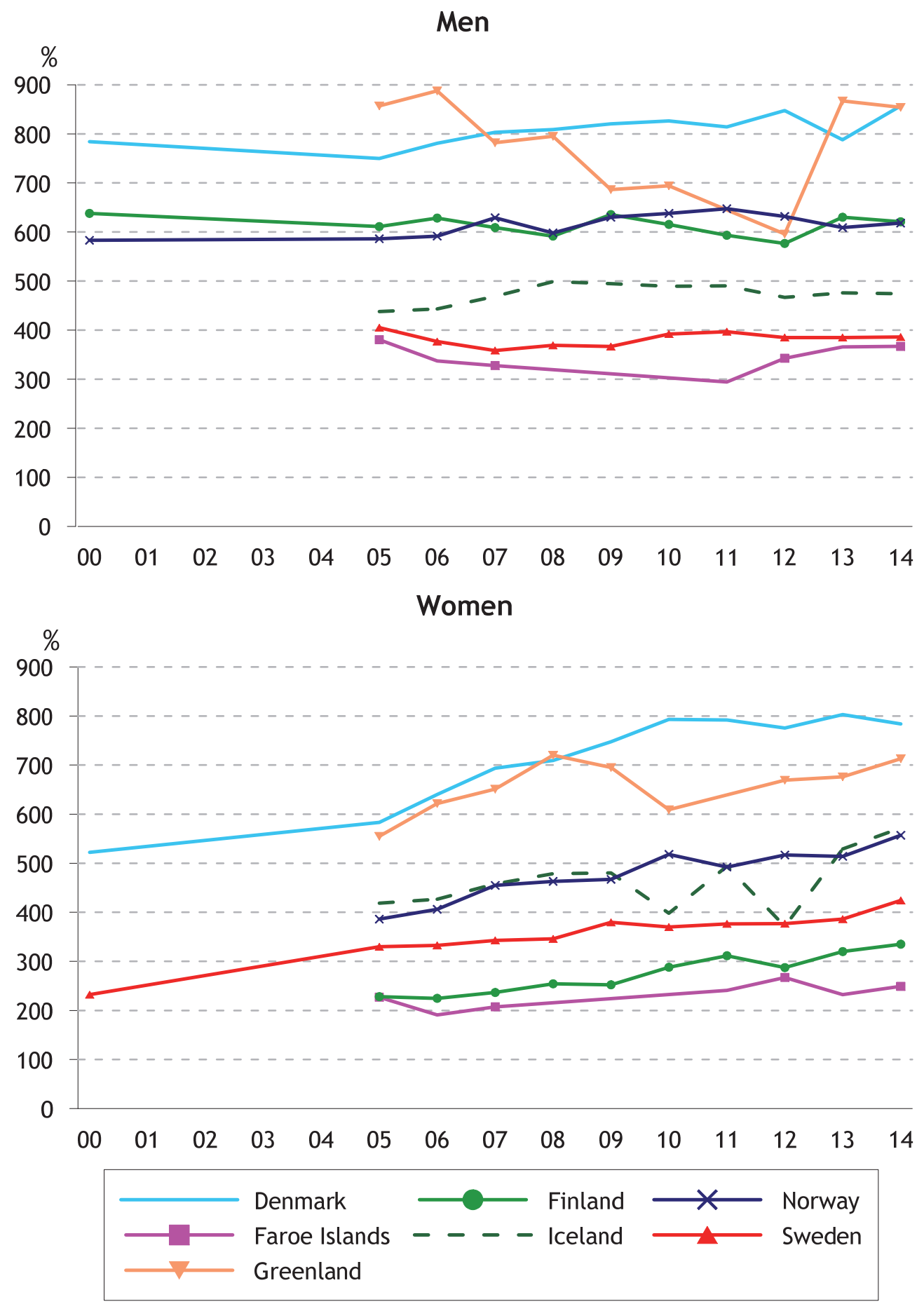
Table 3.1.4 New cases of lung cancer per 1000000 inhabitants, 2000-2014

\begin{tabular}{|c|c|c|c|c|c|c|c|}
\hline & Denmark & $\begin{array}{c}\text { Faroe } \\
\text { Islands }^{1}\end{array}$ & Greenland & Finland & Iceland $^{1}$ & Norway & Sweden \\
\hline \multicolumn{8}{|l|}{ Men } \\
\hline 2000 & 784 & .. & .. & 638 & .. & 583 & 398 \\
\hline 2005 & 750 & .. & 857 & 611 & .. & 586 & 399 \\
\hline 2010 & 820 & .. & 694 & 636 & .. & 638 & 392 \\
\hline 2013 & 788 & 366 & 867 & 630 & 476 & 609 & 385 \\
\hline 2014 & 857 & 367 & 854 & 621 & 474 & 618 & 386 \\
\hline \multicolumn{8}{|c|}{ Women } \\
\hline 2000 & 522 & .. & .. & 283 & .. & 292 & 232 \\
\hline 2005 & 583 & .. & 555 & 228 & .. & 386 & 310 \\
\hline 2010 & 793 & .. & 609 & 288 & .. & 518 & 370 \\
\hline 2013 & 803 & 232 & 676 & 320 & 529 & 514 & 386 \\
\hline 2014 & 784 & 249 & 713 & 335 & 572 & 557 & 424 \\
\hline
\end{tabular}

$12000=1996-00 ; 2005$ = 2000-05; 2010 = 2006-10; 2013 = 2009-13; 2014 = 2010-14

Source: The cancer registers in the Nordic countries; GL, Danish Cancer Society

Table 3.1.5 Sales of drugs used for nicotine dependence (ATC-group N07BA), DDD/1 000 inhabitants/day, 2005-2015

\begin{tabular}{|c|c|c|c|c|c|c|c|c|}
\hline & Denmark & $\begin{array}{l}\text { Faroe } \\
\text { Islands }\end{array}$ & $\begin{array}{l}\text { Green- } \\
\text { land }\end{array}$ & Finland & Åland & Iceland & Norway & Sweden \\
\hline \multicolumn{9}{|c|}{ N07BA01 } \\
\hline \multicolumn{9}{|c|}{ Nicotine } \\
\hline 2005 & 7.6 & 3.7 & 1.7 & 5.3 & 5.6 & 19.4 & 3.7 & 6.7 \\
\hline 2010 & 8.3 & 3.9 & 3.3 & 8.4 & 9.1 & 19.6 & 5.0 & 6.8 \\
\hline 2014 & 8.9 & 4.3 & 1.3 & 10.5 & 10.4 & 22.6 & 6.5 & 7.0 \\
\hline 2015 & 8.9 & 4.5 & .. & 10.8 & 10.6 & 24.5 & 7.0 & 7.0 \\
\hline \multicolumn{9}{|c|}{$\begin{array}{l}\text { N07BA03 } \\
\text { Varenicline }{ }^{1}\end{array}$} \\
\hline 2010 & 0.7 & 1.0 & 0.1 & 0.4 & 0.1 & 1.0 & 0.9 & 0.5 \\
\hline 2014 & 0.2 & 0.4 & 0.1 & 0.2 & 0.2 & 0.8 & 0.5 & 0.4 \\
\hline 2015 & 0.3 & 0.4 & 0.1 & 0.3 & 0.1 & 0.8 & 0.5 & 0.4 \\
\hline
\end{tabular}

1 Varenicline was introduced on the market in December 2006

Source: DK, the Danish Health Data Authority; FO, Chief Pharmaceutical Officer; GL, Central Pharmacy in Copenhagen County; FI \& ÅL, Finnish Medicines Agency; IS, Icelandic Cancer Society; NO, Norwegian Institute of Public Health; SV, Swedish eHealth Agency 
Figure 3.1.3 Percentage of daily smokers by gender, 1990-2014

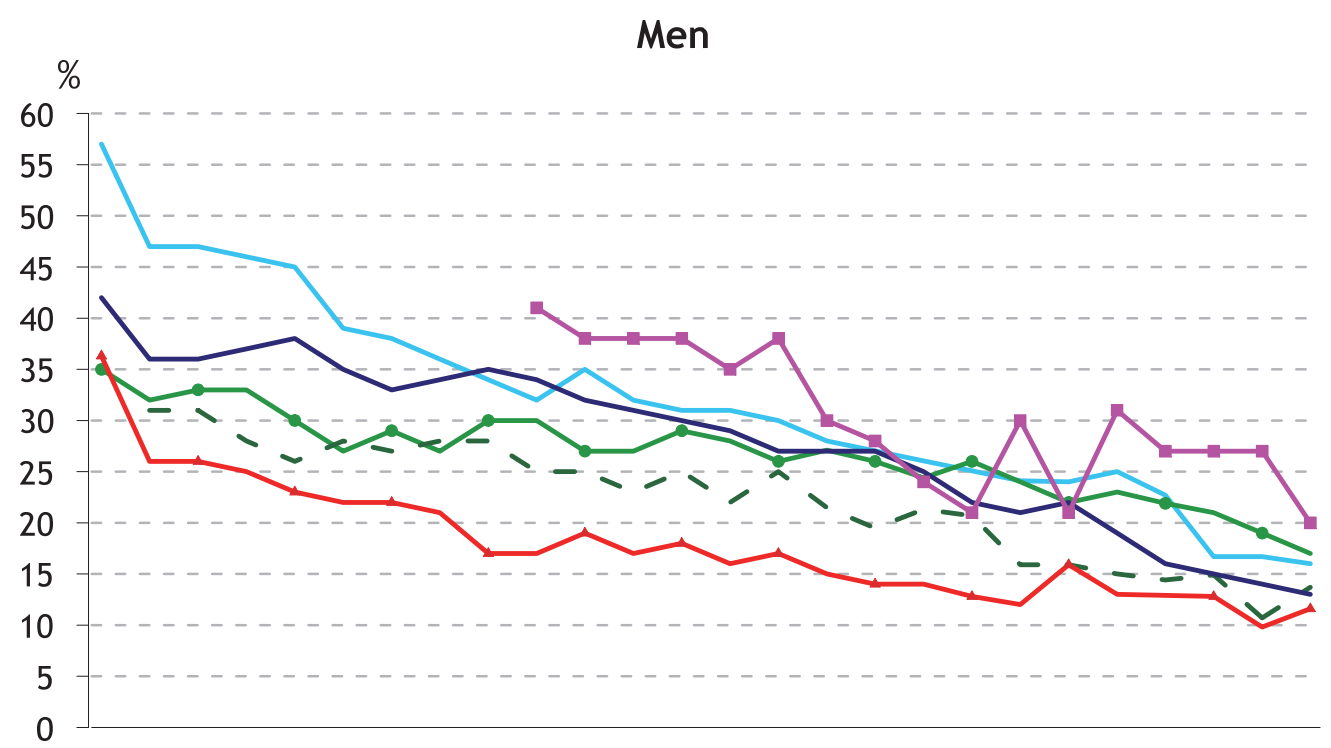

8090919293949596979899000102030405060708091011121314

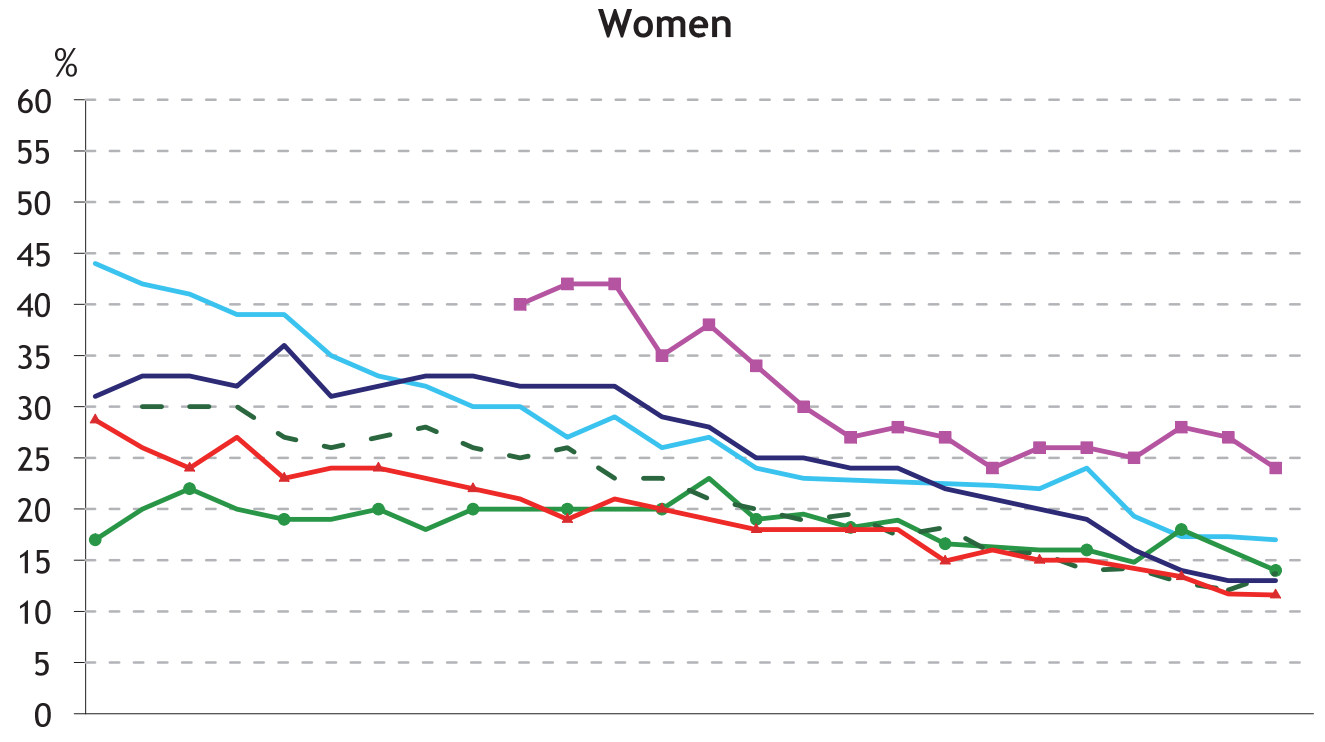

8090919293949596979899000102030405060708091011121314

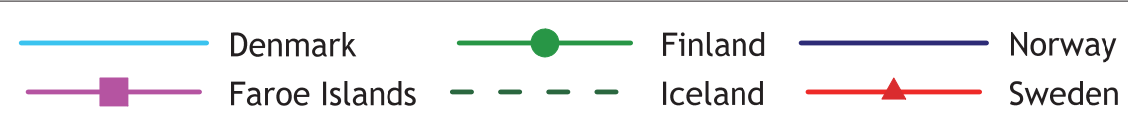

Source: OECD, and National Boards of Health; IS, Directorate of Health (from regular surveys on tobacco consumption); FO, Public Health Council; FI, THL; Health Behaviour and Health among the Finnish Adult Population; SV, Statistics Sweden 
Figure 3.1.4 Sales of drugs used for nicotine dependence (ATC-group N07BA), DDD/1 000 inhabitants/day, 2000-2015

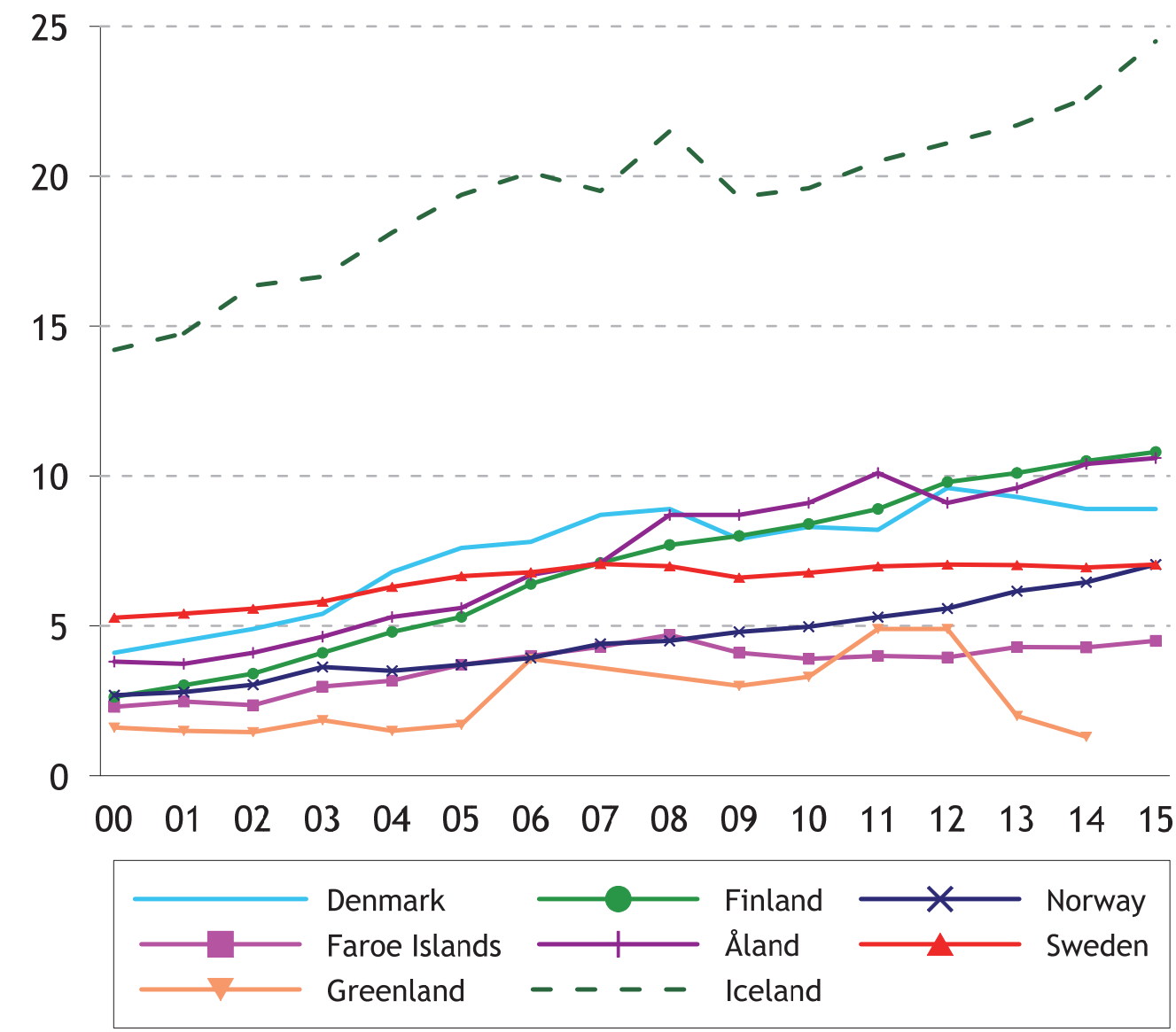

Table 3.1.6 Sales of alcoholic beverages in litres of 100 per cent pure alcohol per inhabitant aged 15 years and over, 2005-2014

\begin{tabular}{lcccccccc}
\hline & Denmark & $\begin{array}{c}\text { Faroe } \\
\text { Islands }\end{array}$ & Greenland & Finland & Åland & Iceland & Norway & Sweden \\
\hline 2000 & 11.5 & 6.8 & 13.6 & 8.6 & 6.1 & 4.8 & 5.6 & 6.2 \\
2005 & 11.3 & 6.6 & 12.1 & 10.0 & 6.6 & 5.7 & 6.4 & 6.6 \\
2010 & 11.3 & 6.7 & 10.4 & 9.7 & 6.8 & 5.5 & 6.6 & 7.3 \\
2013 & 9.4 & 6.3 & 8.8 & 9.1 & 6.8 & 5.3 & 6.2 & 7.4 \\
2014 & 9.5 & 6.3 & 8.6 & 8.8 & 6.6 & 5.3 & 6.1 & 7.3 \\
\hline
\end{tabular}

Source: DK, FO, GL, IS, NO: The central statistical bureaus; FI \& ÅL: THL; SV: Public Health Agency of Sweden 
Figure 3.1.5 Sales of alcoholic beverages in litres of 100 per cent pure alcohol per inhabitant aged 15 years and over, 1995-2014

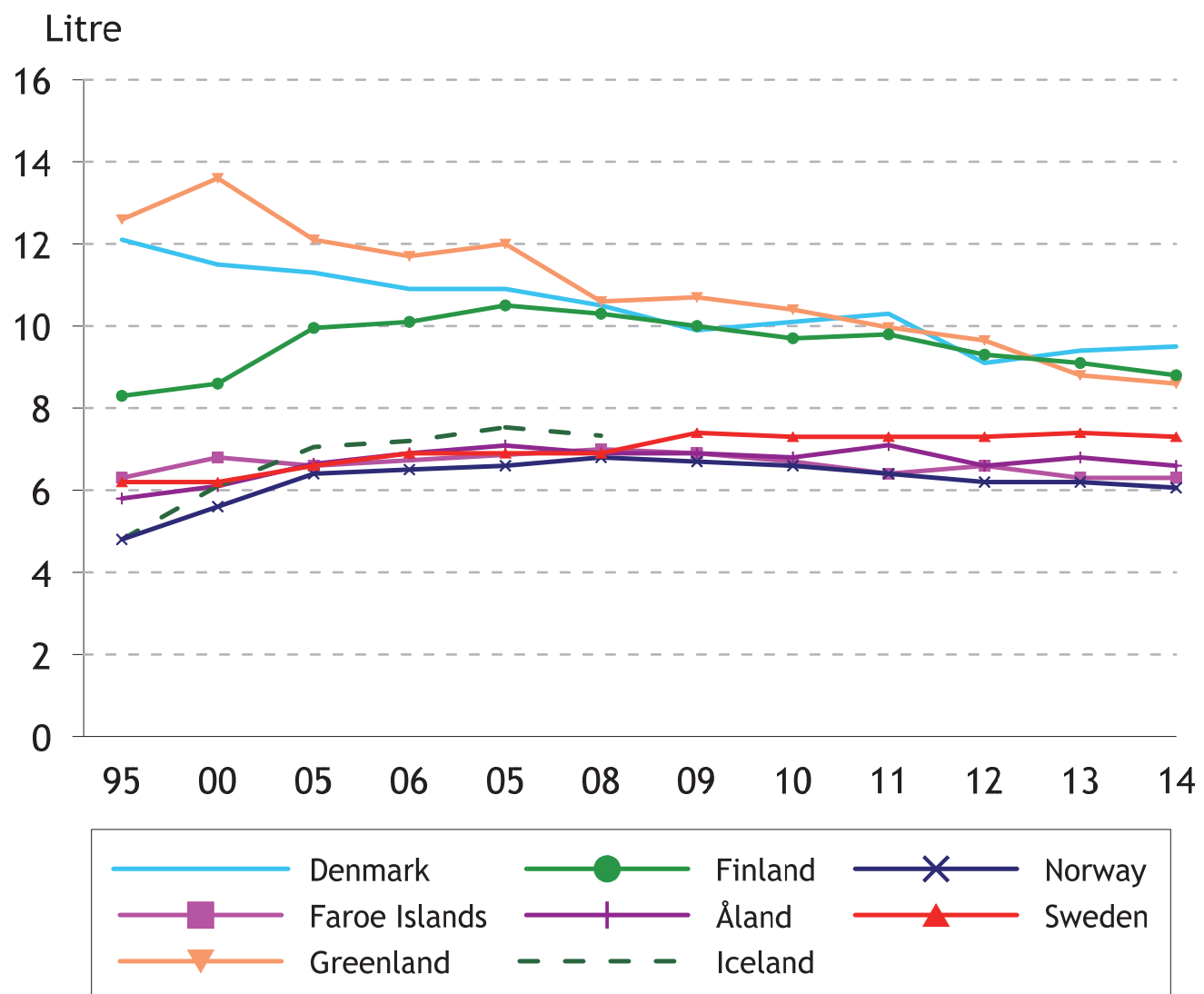

Source: DK, FO, GL, IS, NO: The central statistical bureaus; FI \& ÅL: THL; SV: Public Health Agency of Sweden

Figure 3.1.6 Sales of alcoholic beverages in litres of 100 per cent pure alcohol per inhabitant aged 15 years and over, 2000, 2010 and 2014

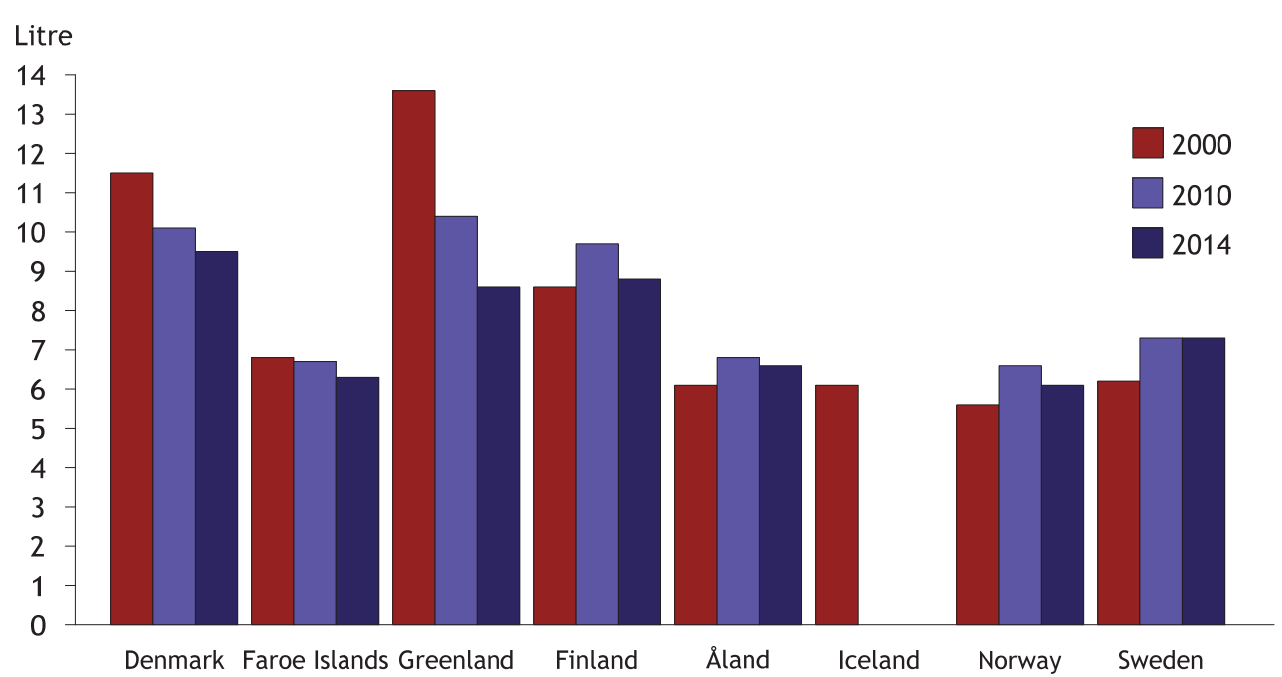

Source: DK, FO, GL, IS, NO: The central statistical bureaus; FI \& ÅL: THL; SV: Public Health Agency of Sweden 
Morbidity, Medical Treatment, Accidents and Pharmaceutical products

Figure 3.1.7 Patients treated in somatic hospitals for alcoholic liver disease (ATC-group K70) per 100000 inhabitants, 2005-2014

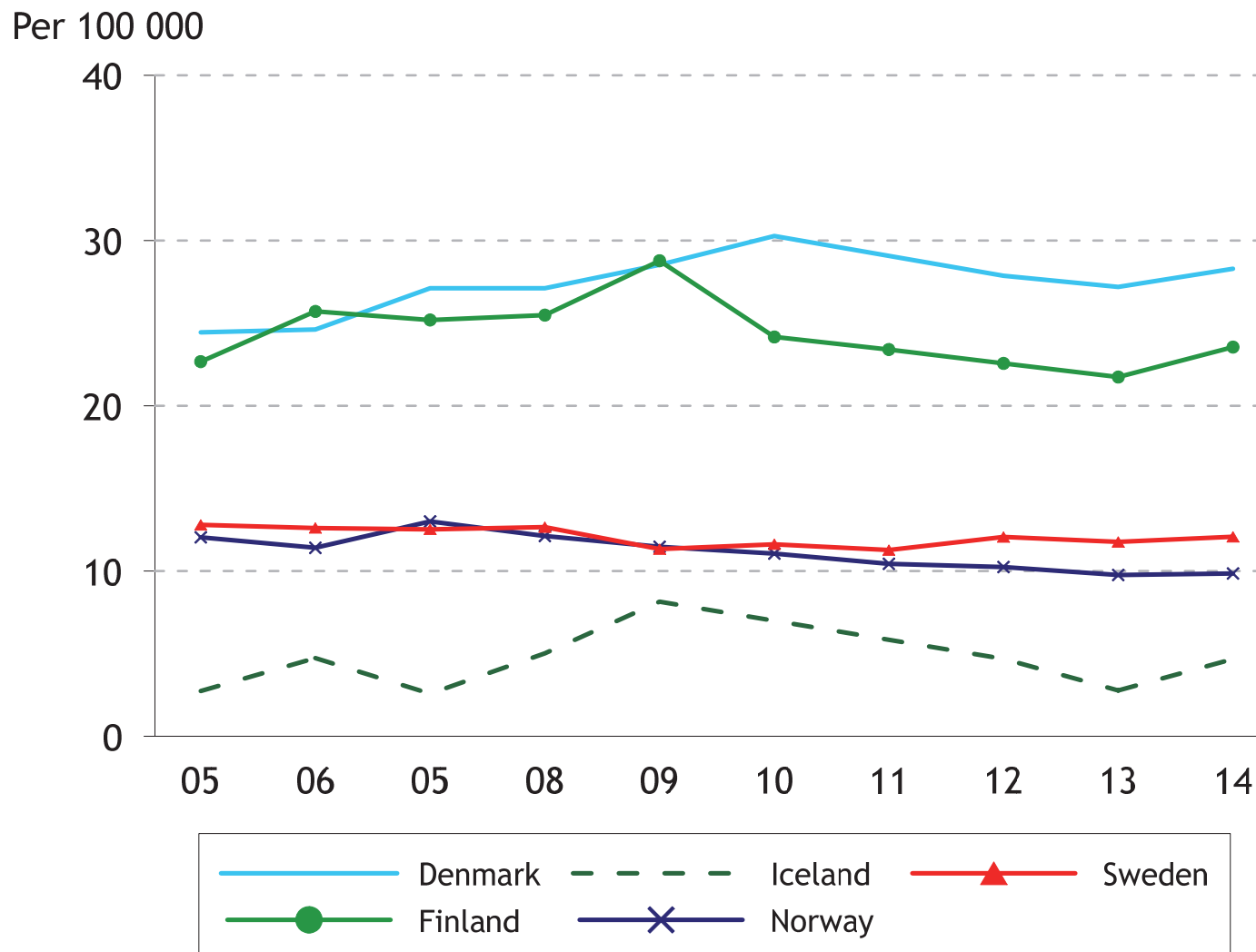

Source: DK, the Danish Health Data Authority; FO, Ministry of Health; FI, THL; IS, Directorate of Health; NO, Norwegian Patient Register; SV, National Board of Health and Welfare 
Figure 3.1.7 Patients treated in somatic hospitals for alcoholic liver disease per 100000 inhabitants, 2014

Per 100000

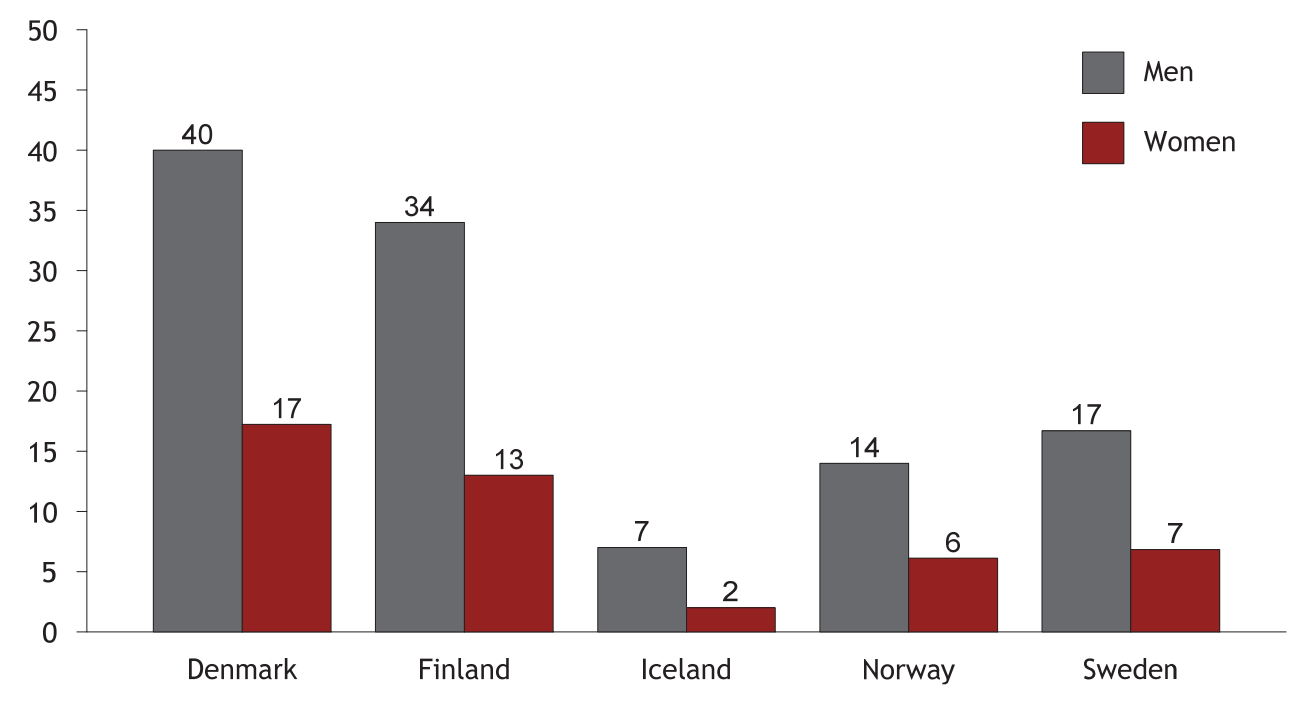

ICD10 K70

Source: DK, the Danish Health Data Authority; FO, Ministry of Health; FI, THL; IS, Directorate of Health; NO, Norwegian Patient Register; SV, National Board of Health and Welfare

Table 3.1.7 Diagnosed cases of tuberculosis per 100000 inhabitants, 2000-2014

\begin{tabular}{lrrrrrrrr}
\hline & Denmark & $\begin{array}{c}\text { Faroe } \\
\text { lslands }\end{array}$ & Greenland & Finland & Åland & Iceland & Norway & Sweden \\
\hline Men & & & & & & & & \\
2000 & 12.1 & 20.8 & 50.0 & 12.4 & 7.9 & 2.8 & 5.8 & 5.2 \\
2005 & 9.5 & - & 178.1 & 8.0 & - & 5.4 & 6.2 & 6.8 \\
2010 & 7.8 & - & 220.5 & 6.9 & 7.2 & 5.0 & 7.4 & 8.0 \\
2013 & 8.3 & 4.2 & 201.1 & 6.2 & - & 3.1 & 8.2 & 7.2 \\
2014 & 6.9 & 2.0 & 232.1 & 6.1 & - & 3.0 & 7.2 & 7.4 \\
Women & & & & & & & & \\
2000 & 8.5 & 4.5 & 111.0 & 8.5 & - & 6.4 & 6.2 & 6.2 \\
2005 & 6.2 & - & 165.1 & 5.8 & 7.5 & 2.0 & 6.1 & 6.1 \\
2010 & 5.3 & 8.7 & 192.3 & 5.0 & - & 8.9 & 6.3 & 6.3 \\
2013 & 4.5 & 4.2 & 135.7 & 3.8 & - & 3.7 & 7.2 & 6.4 \\
2014 & 4.6 & - & 113.1 & 4.2 & - & 2.5 & 5.4 & 6.6 \\
\hline
\end{tabular}

ICD10 A-15-A19

Source: DK, the Danish Health Data Authority; FO, Chief Medical Officer; GL, Chief Medical Officer; FI \& ÅL, THL; IS, Directorate of Health; NO, Norwegian Institute of Public Health; SV, Public Health Agency of Sweden 
Table 3.1.8 Confirmed new cases of HIV/AIDS', 2005-2015

\begin{tabular}{|c|c|c|c|c|c|c|c|c|}
\hline & Denmark & $\begin{array}{l}\text { Faroe } \\
\text { Islands }\end{array}$ & Greenland & Finland & $\begin{array}{c}\text { Of which } \\
\text { Alland }\end{array}$ & Iceland & Norway & Sweden ${ }^{2}$ \\
\hline \multicolumn{9}{|l|}{ Men } \\
\hline 2005 & 192 & - & 4 & 96 & . & 5 & 122 & 228 \\
\hline 2010 & 201 & 1 & 2 & 132 & . & 18 & 173 & 285 \\
\hline 2014 & 180 & - & .. & 135 & . & 8 & 184 & 272 \\
\hline 2015 & 194 & - & 3 & 130 & - & 10 & 145 & 276 \\
\hline \multicolumn{9}{|c|}{ Women } \\
\hline 2005 & 71 & - & 2 & 35 & . & 3 & 97 & 164 \\
\hline 2010 & 72 & - & 1 & 56 & . & 6 & 85 & 180 \\
\hline 2014 & 55 & - & .. & 42 & . & 2 & 65 & 199 \\
\hline 2015 & 66 & - & 2 & 43 & - & 2 & 76 & 174 \\
\hline \multicolumn{9}{|l|}{ Total } \\
\hline 2005 & 263 & - & 6 & 131 & 1 & 8 & 219 & 392 \\
\hline 2010 & 273 & 1 & 3 & 188 & - & 24 & 258 & 465 \\
\hline 2014 & 235 & - & .. & 177 & 1 & 10 & 249 & 473 \\
\hline 2015 & 260 & - & 5 & 173 & - & 12 & 221 & 450 \\
\hline
\end{tabular}

1 From 1985-2000, it was obligatory to report AIDS, which is the end stage of HIV infection. From 2000 reporting of AIDS is voluntary, as a completion to the reporting of HIV. Screening affects the number of newly-reported cases and how many people develop AIDS. Included in the total may be cases where information about gender is missing

2 HIV only

ICD10 B20

Source: DK, the Danish Health Data Authority; FO, Chief Medical Officer; GL, Chief Medical Officer; FI \& $\AA ̊$ L, THL; IS, Directorate of Health; NO, Norwegian Institute of Public Health (MSIS);

SV, Public Health Agency of Sweden 
Figure 3.1.8 Confirmed new cases of HIV/AIDS per 1000000 inhabitants, 20002015

Per 1000000

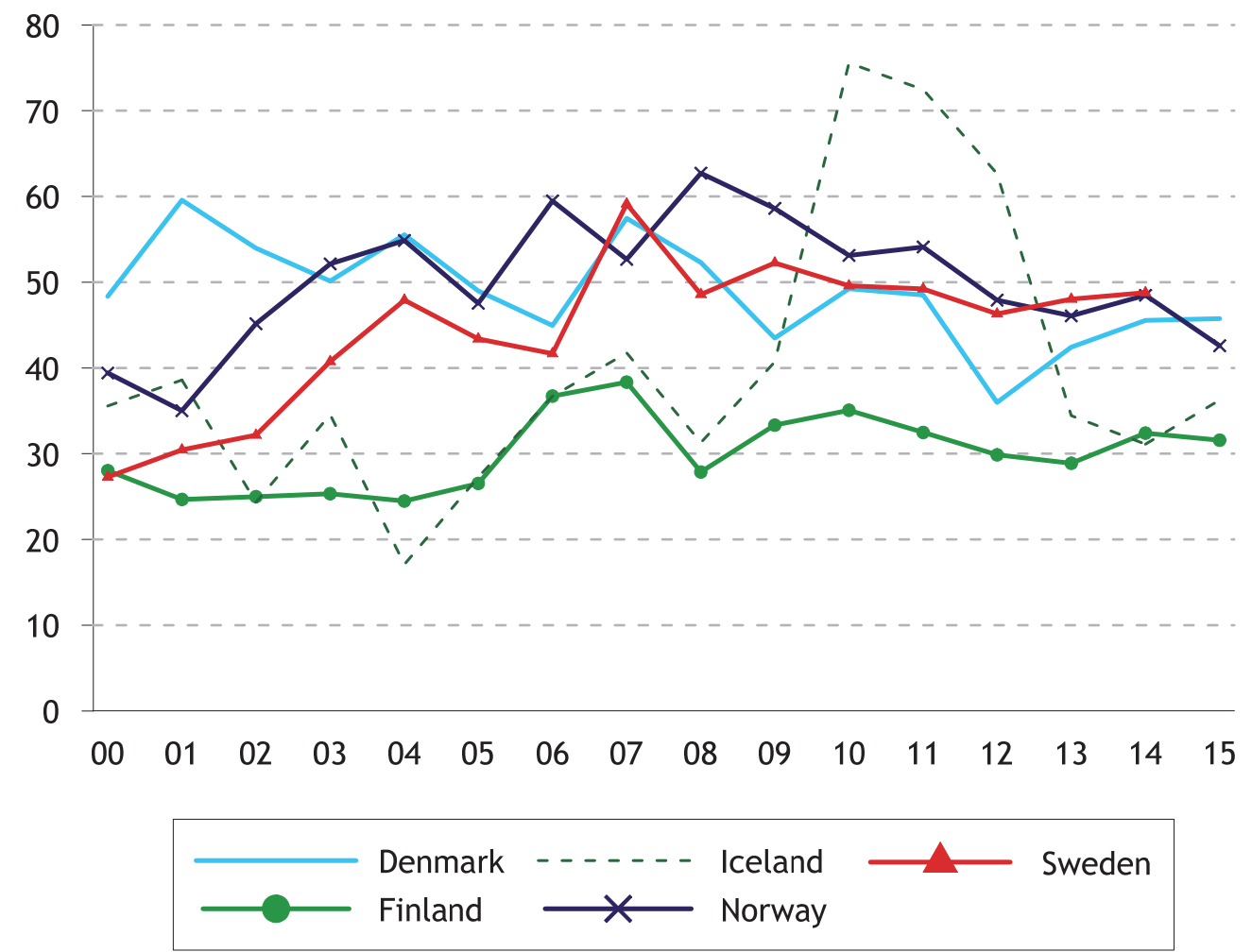

ICD10 B20

Source: DK, the Danish Health Data Authority; FO, Chief Medical Officer; GL, Chief Medical Officer; FI \& ÁL, THL; IS, Directorate of Health; NO, Norwegian Institute of Public Health; SV, Public Health Agency of Sweden 
Table 3.1.9 Notified cases of gonorrhoea per 100000 inhabitants aged 15+

\begin{tabular}{|c|c|c|c|c|c|c|c|c|}
\hline & Denmark & $\begin{array}{l}\text { Faroe } \\
\text { Islands }\end{array}$ & Greenland & Finland & Åland & Iceland & Norway & Sweden \\
\hline \multicolumn{9}{|l|}{ Men } \\
\hline 2005 & 18 & 4 & 1535 & 7 & 8 & 12 & 12 & 16 \\
\hline 2010 & 17 & - & 2307 & 7 & 7 & 7 & 19 & 13 \\
\hline 2013 & 22 & - & 2493 & 7 & - & 10 & 21 & 17 \\
\hline 2014 & 30 & - & 2783 & 8 & 7 & 20 & 27 & 21 \\
\hline \multicolumn{9}{|c|}{ Women } \\
\hline 2005 & 2 & - & 2124 & 2 & - & 4 & 3 & 3 \\
\hline 2010 & 5 & - & 3456 & 2 & - & 3 & 2 & 5 \\
\hline 2013 & 8 & - & 3320 & 3 & - & 5 & 3 & 7 \\
\hline 2014 & 14 & - & 2331 & 3 & 7 & 9 & 6 & 7 \\
\hline
\end{tabular}

Source: DK, the Danish Health Data Authority; FO, Chief Medical Officer; GL, Chief Medical Officer; FI \& ÅL, THL; IS, Directorate of Health; NO, MSIS; SV, Public Health Agency of Sweden

Table 3.1.10 Notified cases of syphilis per 100000 inhabitants aged 15+

\begin{tabular}{|c|c|c|c|c|c|c|c|c|}
\hline & Denmark & $\begin{array}{l}\text { Faroe } \\
\text { Islands }\end{array}$ & Greenland & Finland & Åland & Iceland & Norway & Sweden \\
\hline \multicolumn{9}{|l|}{ Men } \\
\hline 2005 & 5 & - & 3 & 3 & 8 & 3 & 6 & 2 \\
\hline 2010 & 16 & - & .. & 5 & - & 2 & 6 & 3 \\
\hline 2013 & 12 & 2 & 68 & 4 & 7 & 2 & 8 & 5 \\
\hline 2014 & 17 & - & 61 & 5 & - & 19 & 8 & 4 \\
\hline \multicolumn{9}{|c|}{ Women } \\
\hline 2005 & 1 & - & 4 & 2 & - & 1 & - & - \\
\hline 2010 & 2 & - & .. & 3 & - & 1 & 1 & 1 \\
\hline 2013 & 1 & - & 83 & 2 & - & 1 & 1 & 1 \\
\hline 2014 & 2 & 1 & 36 & 3 & - & .. & 1 & 1 \\
\hline
\end{tabular}

Source: DK, the Danish Health Data Authority; FO, Chief Medical Officer; GL, Chief Medical Officer; FI \& $\AA ̊$ L, THL; IS, Directorate of Health; NO, Norwegian Institute of Public Health (MSIS); SV, Public Health Agency of Sweden

Table 3.1.11 Diagnosed cases of Chlamydia per 100000 inhabitants

\begin{tabular}{lcccccccc}
\hline & Denmark & $\begin{array}{c}\text { Faroe } \\
\text { Islands }\end{array}$ & Greenland & Finland & Åland & Iceland ${ }^{1}$ & Norway & Sweden $^{2}$ \\
\hline Men & & & & & & & & \\
2000 & 165 & 79 & 2791 & 180 & 95 & 479 & 326 & 187 \\
2005 & 324 & 231 & 3852 & 197 & 221 & 412 & 330 & 317 \\
2010 & 383 & 286 & 5277 & 202 & 196 & 551 & 351 & 340 \\
2013 & 388 & 143 & 5282 & 203 & 267 & 552 & 364 & 321 \\
2014 & 435 & 110 & 4440 & 207 & 84 & 405 & 386 & 321 \\
Women & & & & & & & & \\
2000 & 384 &.. & 4817 & 272 & 207 & 781 & & 246 \\
2005 & 554 &.. & 5797 & 288 & 499 & 643 & 524 & 411 \\
2010 & 622 & 403 & 8762 & 276 & 251 & 852 & 567 & 445 \\
2013 & 595 & 196 & 9731 & 282 & 294 & 769 & 539 & 425 \\
2014 & 659 & 180 & 8045 & 280 & 112 & 627 & 580 & 424 \\
\hline
\end{tabular}

1 Notified cases. Since 1997, cases verified by laboratories. The total (men and women) includes cases with missing data about gender

2 Possible underrepresentation in 2005-06, due to a mutated form of chlamydia, which was diagnosable at the time

Source: DK, the Danish Health Data Authority; FO, Chief Medical Officer; GL, Chief Medical Officer; FI \& ÅL, THL; IS, Directorate of Health; NO, Institute of Public Health (MSIS); SV, Public Health Agency of Sweden 


\subsection{Cancer}

All the Nordic countries have population-based cancer registers and all the countries except Sweden have centralized coding and classification.

Both external and internal factors that produce changes in the DNA material can cause cancer. Stimulants, foodstuffs, exposure to some occupational hazards and factors in the environment have been shown to be cancer inducing.

The incidence of cancer increases with age. The annual number of cases of cancer is increasing in all the Nordic countries, and this trend remains after adjusting for differences in the size and age structure of the population.

The development of cancer diseases in the Nordic countries remains analogous for most forms of cancer, but there are interesting differences. In general, the number of cases has increased with time, with a few exceptions of decreasing incidence such as for cancer of the stomach. The decrease in the incidence of cancer of the cervix in the Nordic countries is related to the public screening programmes to detect precancerous lesions and early lesions, and the ensuing treatment.

The incidence of breast cancer, cancer of the prostate and colorectal cancer is increasing in almost all countries. Dietary factors are probably significant for this development, but for cancer of the breast and prostate, hormonal factors also play an important role. The incidence of cancer of the testis is again increasing in most of the countries. The incidence of tobacco-related cancers, such as lung cancer, is high in all the countries. However, the incidence of lung cancer among men is decreasing. 
Morbidity, Medical Treatment, Accidents and Pharmaceutical products

Table 3.2.1.a New cases of cancer per 1000000 inhabitants, men

\begin{tabular}{|c|c|c|c|c|c|c|c|c|}
\hline & Total $^{1}$ & $\begin{array}{c}\text { C62 } \\
\text { Testis }\end{array}$ & $\begin{array}{c}\text { C61 } \\
\text { Prostate }\end{array}$ & $\begin{array}{c}\text { C16 } \\
\text { Stomach }\end{array}$ & $\begin{array}{l}\text { C18-21 } \\
\text { Colon and } \\
\text { rectum }\end{array}$ & $\begin{array}{c}\text { C25 } \\
\text { Pancreas }\end{array}$ & $\begin{array}{l}\text { C33-34 } \\
\text { Lungs }\end{array}$ & $\begin{array}{c}\text { C43 } \\
\text { Melanoma } \\
\text { of the skin }\end{array}$ \\
\hline \multicolumn{9}{|l|}{ Denmark } \\
\hline 2005 & 6534 & 99 & 1597 & 123 & 918 & 163 & 818 & 340 \\
\hline 2010 & 5923 & 117 & 1425 & 144 & 848 & 171 & 820 & 310 \\
\hline 2013 & 6293 & 95 & 1538 & 118 & 884 & 157 & 788 & 327 \\
\hline 2014 & 6768 & 99 & 1634 & 144 & 1049 & 175 & 857 & 358 \\
\hline \multicolumn{9}{|c|}{ Faroe Islands } \\
\hline 2005-09 & 3484 & 174 & 1148 & 71 & 515 & 150 & 285 & 103 \\
\hline $2010-14$ & 3779 & 96 & 1076 & 120 & 638 & 191 & 367 & 80 \\
\hline \multicolumn{9}{|c|}{ Greenland } \\
\hline 2005-09 & 2846 & 47 & 153 & 133 & 347 & 147 & 687 & 27 \\
\hline $2010-14$ & 3291 & 27 & 227 & 234 & 427 & 154 & 854 & 47 \\
\hline \multicolumn{9}{|l|}{ Finland } \\
\hline 2005 & 5282 & 53 & 2076 & 152 & 495 & 165 & 628 & 160 \\
\hline 2010 & 5391 & 49 & 1753 & 149 & 530 & 192 & 636 & 240 \\
\hline 2013 & 5804 & 65 & 1886 & 131 & 582 & 190 & 630 & 273 \\
\hline 2014 & 5576 & 65 & 1698 & 138 & 603 & 197 & 621 & 255 \\
\hline \multicolumn{9}{|l|}{ Åland } \\
\hline 2005-09 & 6939 & 45 & 3008 & 194 & 551 & 283 & 670 & 238 \\
\hline $2010-14$ & 6663 & 71 & 2611 & 127 & 649 & 198 & 565 & 353 \\
\hline \multicolumn{9}{|l|}{ Iceland } \\
\hline 2005-09 & 4329 & 66 & 1399 & 125 & 474 & 78 & 495 & 135 \\
\hline $2010-14$ & 4303 & 56 & 1273 & 102 & 486 & 113 & 474 & 108 \\
\hline \multicolumn{9}{|l|}{ Norway } \\
\hline 2005 & 5574 & 109 & 1592 & 127 & 750 & 124 & 586 & 249 \\
\hline 2010 & 6183 & 111 & 1723 & 125 & 836 & 129 & 638 & 304 \\
\hline 2013 & 6097 & 132 & 1893 & 117 & 828 & 138 & 609 & 328 \\
\hline 2014 & 6201 & 124 & 1893 & 117 & 845 & 139 & 618 & 393 \\
\hline \multicolumn{9}{|l|}{ Sweden } \\
\hline 2005 & 5557 & 63 & 2207 & 129 & 635 & 100 & 405 & 242 \\
\hline 2010 & 5560 & 64 & 2077 & 110 & 690 & 111 & 392 & 314 \\
\hline 2013 & 5798 & 77 & 2020 & 117 & 691 & 126 & 385 & 354 \\
\hline 2014 & 6063 & 76 & 2268 & 106 & 694 & 134 & 386 & 395 \\
\hline
\end{tabular}

Source: The cancer registers in the Nordic countries; GL, Danish Cancer Society

1 ICD10 Chapter C, except C44 and C46.0, incl. D09-0, D32, D33, D41.4 and D43 
Morbidity, Medical Treatment, Accidents and Pharmaceutical products

Table 3.2.1.b New cases of cancer, age-standardized rates per 1000000 men (Nordic population 2000)

\begin{tabular}{|c|c|c|c|c|c|c|c|c|}
\hline & Total $^{1}$ & $\begin{array}{c}\text { C62 } \\
\text { Testis }\end{array}$ & $\begin{array}{c}\text { C61 } \\
\text { Prostate }\end{array}$ & $\begin{array}{c}\text { C16 } \\
\text { Stomach }\end{array}$ & $\begin{array}{c}\text { C18-21 } \\
\text { Colon and } \\
\text { rectum }\end{array}$ & $\begin{array}{c}\text { C25 } \\
\text { Pancreas }\end{array}$ & $\begin{array}{l}\text { C33-34 } \\
\text { Lungs }\end{array}$ & $\begin{array}{c}\text { C43 } \\
\text { Melanoma } \\
\text { of the skin }\end{array}$ \\
\hline \multicolumn{9}{|l|}{ Denmark } \\
\hline 2005 & 5661 & 103 & 1249 & 140 & 845 & 172 & 856 & 239 \\
\hline 2010 & 6099 & 92 & 1445 & 139 & 887 & 177 & 831 & 303 \\
\hline 2013 & 5959 & 100 & 1408 & 115 & 858 & 149 & 746 & 313 \\
\hline 2014 & 6293 & 103 & 1460 & 139 & 992 & 161 & 791 & 339 \\
\hline \multicolumn{9}{|c|}{ Faroe Islands } \\
\hline 2005-09 & 3888 & 189 & 1282 & 91 & 611 & 162 & 322 & 108 \\
\hline $2010-14$ & 3923 & 103 & 1095 & 129 & 663 & 199 & 382 & 86 \\
\hline \multicolumn{9}{|c|}{ Greenland } \\
\hline 2005-09 & 4878 & 87 & 503 & 224 & 702 & 294 & 2225 & 29 \\
\hline $2010-14$ & 5145 & 21 & 385 & 325 & 839 & 245 & 1344 & 57 \\
\hline \multicolumn{9}{|l|}{ Finland } \\
\hline 2005 & 5842 & 53 & 2282 & 178 & 552 & 186 & 706 & 170 \\
\hline 2010 & 5095 & 47 & 1668 & 136 & 519 & 184 & 586 & 212 \\
\hline 2013 & 5415 & 67 & 1703 & 123 & 549 & 182 & 584 & 259 \\
\hline 2014 & 5093 & 68 & 1502 & 130 & 552 & 181 & 562 & 239 \\
\hline \multicolumn{9}{|l|}{ Åland } \\
\hline 2005-09 & 6578 & 47 & 2782 & 157 & 519 & 274 & 613 & 228 \\
\hline $2010-14$ & 5865 & 72 & 2205 & 114 & 572 & 168 & 522 & 323 \\
\hline \multicolumn{9}{|l|}{ Iceland } \\
\hline 2005-09 & 5533 & 63 & 1838 & 160 & 608 & 101 & 654 & 159 \\
\hline $2010-14$ & 5029 & 56 & 1505 & 125 & 571 & 132 & 575 & 123 \\
\hline \multicolumn{9}{|l|}{ Norway } \\
\hline 2005 & 6336 & 109 & 1824 & 161 & 861 & 139 & 662 & 276 \\
\hline 2010 & 6764 & 111 & 1859 & 139 & 927 & 144 & 704 & 321 \\
\hline 2013 & 6448 & 131 & 1963 & 126 & 896 & 151 & 654 & 345 \\
\hline 2014 & 6476 & 122 & 1933 & 126 & 907 & 146 & 654 & 413 \\
\hline \multicolumn{9}{|l|}{ Sweden } \\
\hline 2005 & 5362 & 64 & 2101 & 126 & 618 & 97 & 391 & 236 \\
\hline 2010 & 5155 & 65 & 1874 & 102 & 656 & 101 & 361 & 298 \\
\hline 2013 & 5236 & 79 & 1759 & 108 & 634 & 113 & 343 & 331 \\
\hline 2014 & 5281 & 77 & 1903 & 94 & 612 & 116 & 330 & 359 \\
\hline
\end{tabular}

Source: The cancer registers in the Nordic countries; GL, Danish Cancer Society

1 ICD10 Chapter C, except C44 and C46.0, incl. D09-0, D32, D33, D41.4, D42 and D43 
Morbidity, Medical Treatment, Accidents and Pharmaceutical products

Table 3.2.2.a New cases of cancer, per 1000000 inhabitants, women

\begin{tabular}{|c|c|c|c|c|c|c|c|c|}
\hline & Total $^{1}$ & $\begin{array}{l}\text { C50 } \\
\text { Breast }\end{array}$ & $\begin{array}{c}\text { C53 } \\
\text { Cervix } \\
\text { uteri }\end{array}$ & $\begin{array}{c}\text { C16 } \\
\text { Stomach }\end{array}$ & $\begin{array}{l}\text { C18-21 } \\
\text { Colon and } \\
\text { rectum }\end{array}$ & $\begin{array}{c}\text { C25 } \\
\text { Pancreas }\end{array}$ & $\begin{array}{l}\text { C33-34 } \\
\text { Lungs }\end{array}$ & $\begin{array}{c}\text { C43 } \\
\text { Melanoma } \\
\text { of the skin }\end{array}$ \\
\hline \multicolumn{9}{|c|}{ Denmark } \\
\hline 2005 & 6257 & 1725 & 135 & 61 & 781 & 174 & 829 & 396 \\
\hline 2010 & 6137 & 1842 & 130 & 65 & 765 & 164 & 793 & 345 \\
\hline 2013 & 6057 & 1670 & 131 & 59 & 756 & 168 & 803 & 383 \\
\hline 2014 & 6207 & 1663 & 145 & 63 & 886 & 164 & 784 & 414 \\
\hline \multicolumn{9}{|c|}{ Faroe Islands } \\
\hline 2005-09 & 3426 & 839 & 137 & 111 & 548 & 111 & 248 & 257 \\
\hline $2010-14$ & 3340 & 936 & 112 & 86 & 507 & 103 & 249 & 86 \\
\hline \multicolumn{9}{|c|}{ Greenland } \\
\hline 2005-09 & 3692 & 545 & 366 & 103 & 301 & 160 & 695 & 47 \\
\hline $2010-14$ & 3685 & 578 & 218 & 113 & 495 & 158 & 713 & 53 \\
\hline \multicolumn{9}{|l|}{ Finland } \\
\hline 2005 & 4449 & 1505 & 47 & 101 & 452 & 176 & 225 & 140 \\
\hline 2010 & 5270 & 1779 & 53 & 100 & 503 & 195 & 288 & 243 \\
\hline 2013 & 5350 & 1739 & 58 & 96 & 539 & 200 & 320 & 253 \\
\hline 2014 & 5386 & 1795 & 63 & 97 & 508 & 216 & 335 & 239 \\
\hline \multicolumn{9}{|l|}{ Åland } \\
\hline 2005-09 & 5243 & 1509 & 44 & 161 & 571 & 249 & 395 & 395 \\
\hline $2010-14$ & 5600 & 1656 & 70 & 98 & 702 & 154 & 393 & 379 \\
\hline \multicolumn{9}{|l|}{ Iceland } \\
\hline 2005-09 & 4075 & 1276 & 89 & 77 & 418 & 89 & 480 & 194 \\
\hline $2010-14$ & 4103 & 1262 & 95 & 79 & 411 & 90 & 572 & 150 \\
\hline \multicolumn{9}{|l|}{ Norway } \\
\hline 2005 & 4978 & 1198 & 126 & 97 & 736 & 124 & 386 & 243 \\
\hline 2010 & 5382 & 1161 & 132 & 72 & 748 & 137 & 518 & 317 \\
\hline 2013 & 5180 & 1272 & 111 & 63 & 820 & 150 & 514 & 348 \\
\hline 2014 & 5655 & 1301 & 132 & 73 & 810 & 147 & 557 & 387 \\
\hline \multicolumn{9}{|l|}{ Sweden } \\
\hline 2005 & 5602 & 1529 & 94 & 74 & 647 & 97 & 330 & 228 \\
\hline 2010 & 5877 & 1682 & 91 & 69 & 637 & 103 & 370 & 287 \\
\hline 2013 & 6611 & 1897 & 97 & 64 & 645 & 124 & 386 & 346 \\
\hline 2014 & 6781 & 2005 & 113 & 64 & 636 & 134 & 424 & 379 \\
\hline
\end{tabular}

Source: The cancer registers in the Nordic countries; GL, Danish Cancer Society

1 ICD10 Chapter C, except C44 and C46.0, incl. D09-0, D32, D33, D41.4, D42 and D43 
Table 3.2.2.b New cases of cancer, age-standardized rates per 1000000 women (Nordic population 2000)

\begin{tabular}{|c|c|c|c|c|c|c|c|c|}
\hline & Total $^{1}$ & $\begin{array}{c}\text { C50 } \\
\text { Breast }\end{array}$ & $\begin{array}{c}\text { C53 } \\
\text { Cervix } \\
\text { uteri } \\
\end{array}$ & $\begin{array}{c}\text { C16 } \\
\text { Stomach }\end{array}$ & $\begin{array}{l}\text { C18-21 } \\
\text { Colon and } \\
\text { rectum }\end{array}$ & $\begin{array}{c}\text { C25 } \\
\text { Pancreas }\end{array}$ & $\begin{array}{l}\text { C33-34 } \\
\text { Lungs }\end{array}$ & $\begin{array}{c}\text { C43 } \\
\text { Melanoma } \\
\text { of the skin }\end{array}$ \\
\hline \multicolumn{9}{|c|}{ Denmark } \\
\hline 2005 & 4894 & 1350 & 148 & 57 & 628 & 133 & 619 & 268 \\
\hline 2010 & 5357 & 1619 & 126 & 56 & 647 & 139 & 679 & 324 \\
\hline 2013 & 5245 & 1469 & 129 & 49 & 630 & 138 & 672 & 358 \\
\hline 2014 & 5329 & 1461 & 144 & 53 & 730 & 132 & 641 & 384 \\
\hline \multicolumn{9}{|c|}{ Faroe Islands } \\
\hline 2005-09 & 3387 & 818 & 151 & 105 & 527 & 103 & 252 & 287 \\
\hline $2010-14$ & 3094 & 863 & 105 & 73 & 461 & 98 & 238 & 90 \\
\hline \multicolumn{9}{|c|}{ Greenland } \\
\hline 2005-09 & 4616 & 506 & 330 & 143 & 574 & 220 & 953 & 32 \\
\hline $2010-14$ & 5156 & 706 & 239 & 204 & 743 & 222 & 1175 & 55 \\
\hline \multicolumn{9}{|l|}{ Finland } \\
\hline 2005 & 3864 & 1347 & 45 & 86 & 379 & 146 & 185 & 127 \\
\hline 2010 & 4037 & 1430 & 49 & 69 & 358 & 134 & 208 & 196 \\
\hline 2013 & 4250 & 1433 & 57 & 71 & 407 & 145 & 234 & 221 \\
\hline 2014 & 4267 & 1481 & 61 & 72 & 378 & 155 & 244 & 206 \\
\hline \multicolumn{9}{|l|}{ Åland } \\
\hline 2005-09 & 4304 & 1295 & 42 & 136 & 429 & 182 & 318 & 200 \\
\hline $2010-14$ & 3910 & 1253 & 60 & 59 & 446 & 89 & 323 & 313 \\
\hline \multicolumn{9}{|l|}{ Iceland } \\
\hline 2005-09 & 4485 & 1418 & 92 & 83 & 456 & 98 & 541 & 200 \\
\hline $2010-14$ & 4324 & 1330 & 98 & 76 & 430 & 97 & 625 & 155 \\
\hline \multicolumn{9}{|l|}{ Norway } \\
\hline 2005 & 4661 & 1173 & 125 & 85 & 658 & 108 & 373 & 235 \\
\hline 2010 & 4996 & 1116 & 132 & 62 & 668 & 120 & 486 & 302 \\
\hline 2013 & 4801 & 1216 & 112 & 56 & 733 & 134 & 472 & 331 \\
\hline 2014 & 4961 & 1234 & 134 & 65 & 727 & 130 & 507 & 364 \\
\hline \multicolumn{9}{|l|}{ Sweden } \\
\hline 2005 & 4957 & 1358 & 90 & 58 & 518 & 81 & 281 & 205 \\
\hline 2010 & 5182 & 1480 & 88 & 56 & 509 & 86 & 304 & 256 \\
\hline 2013 & 5829 & 1660 & 96 & 51 & 512 & 99 & 308 & 310 \\
\hline 2014 & 5887 & 1719 & 111 & 50 & 490 & 104 & 332 & 336 \\
\hline
\end{tabular}

Source: The cancer registers in the Nordic countries; GL, Danish Cancer Society

1 ICD10 Chapter C, except C44 and C46.0, incl. D09-0, D32, D33, D41.4, D42 and D43 
Morbidity, Medical Treatment, Accidents and Pharmaceutical products

Table 3.2.3 New cases of leukaemia per 1000000 inhabitants, 0-14 year-olds

\begin{tabular}{|c|c|c|c|c|c|c|}
\hline & Denmark & Finland & Åland 1 & Iceland & Norway & Sweden \\
\hline Boys & & & $M+W$ & & & \\
\hline 2005 & 67 & 47 & . & 24 & 37 & 62 \\
\hline 2010 & 55 & 48 & - & 53 & 37 & 75 \\
\hline 2013 & 70 & 68 & - & 53 & 69 & 69 \\
\hline 2014 & 40 & 48 & - & 47 & 48 & 57 \\
\hline \multicolumn{7}{|l|}{ Girls } \\
\hline 2005 & 63 & 56 & . & 31 & 32 & 44 \\
\hline 2010 & 58 & 20 & - & 37 & 36 & 63 \\
\hline 2013 & 65 & 53 & - & 46 & 40 & 42 \\
\hline 2014 & 38 & 55 & - & 37 & 42 & 45 \\
\hline \multicolumn{7}{|c|}{ Total $^{2}$} \\
\hline 2005 & 65 & 51 & 42 & 27 & 34 & 53 \\
\hline 2010 & 56 & 17 & - & 45 & 36 & 69 \\
\hline 2013 & 68 & 60 & - & 53 & 55 & 56 \\
\hline 2014 & 39 & 51 & - & 42 & 45 & 51 \\
\hline
\end{tabular}

$12000=1996-00 ; 2005=2000-05 ; 2010=2006-10 ; 2013=2009-13 ; 2014=2010-14$ ICD10 C91-C95

Source: The cancer registers in the Nordic countries; GL, Danish Cancer Society

Table 3.2.4 New cases of cancer of the colon and rectum per 1000000 inhabitants

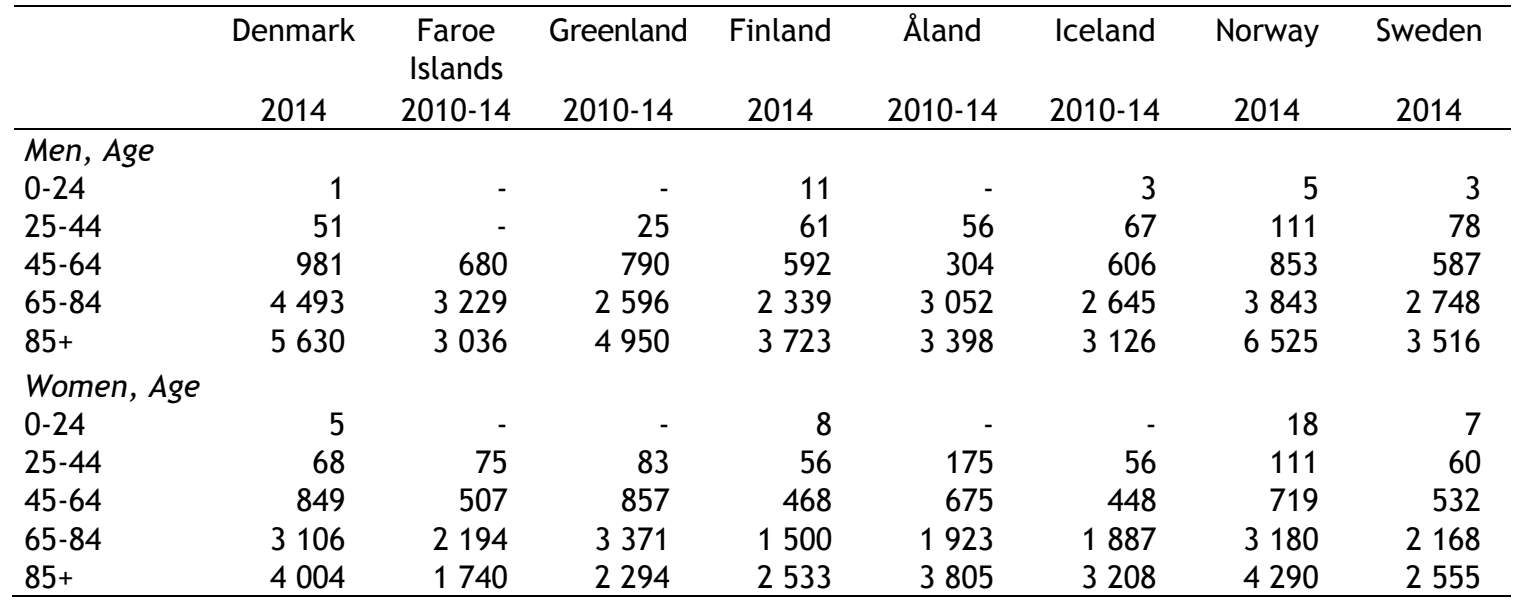

ICD10 C18-21

Source: The cancer registers in the Nordic countries; GL, Danish Cancer Society 
Table 3.2.5 New cases of lung cancer per 1000000 inhabitants

\begin{tabular}{|c|c|c|c|c|c|c|c|c|}
\hline & Denmark & $\begin{array}{l}\text { Faroe } \\
\text { Islands }\end{array}$ & Greenland & Finland & Åland & Iceland & Norway & Sweden \\
\hline & 2014 & 2010-14 & 2010-14 & 2014 & 2010-14 & 2010-14 & 2014 & 2014 \\
\hline \multicolumn{9}{|c|}{ Men, age } \\
\hline $0-24$ & 1 & - & - & - & - & - & - & - \\
\hline $25-44$ & 27 & - & 49 & 23 & - & 18 & 10 & 16 \\
\hline $45-64$ & 745 & 340 & 1196 & 522 & 608 & 155 & 572 & 291 \\
\hline $65-84$ & 3892 & 2071 & 7019 & 2630 & 2093 & 2809 & 3148 & 1736 \\
\hline $85+$ & 3420 & 607 & - & 4015 & 3398 & 2918 & 4135 & 1067 \\
\hline \multicolumn{9}{|c|}{ Women, age } \\
\hline $0-24$ & 2 & - & - & - & - & 4 & 3 & - \\
\hline $25-44$ & 34 & 38 & 56 & 12 & 58 & 32 & 22 & 11 \\
\hline $45-64$ & 799 & 473 & 971 & 285 & 482 & 698 & 646 & 432 \\
\hline $65-84$ & 3022 & 792 & 6109 & 1177 & 1421 & 3110 & 2348 & 1591 \\
\hline $85+$ & 1653 & 290 & 2294 & 1043 & - & 1392 & 1499 & 712 \\
\hline
\end{tabular}

ICD10 C33-34

Source: The cancer registers in the Nordic countries; GL, Danish Cancer Society

Table 3.2.6 New cases of cancer of the cervix uteri per 1000000 women

\begin{tabular}{|c|c|c|c|c|c|c|c|c|}
\hline & Denmark & $\begin{array}{l}\text { Faroe } \\
\text { Islands }\end{array}$ & $\begin{array}{l}\text { Green- } \\
\text { land }\end{array}$ & Finland & Åland & Iceland & Norway & Sweden \\
\hline & 2014 & 2010-14 & 2010-14 & 2014 & $2010-14$ & 2010-14 & 2014 & 2014 \\
\hline \multicolumn{9}{|l|}{ Age } \\
\hline $0-24$ & 13 & - & 57 & 3 & - & 11 & 8 & 11 \\
\hline $25-44$ & 235 & 113 & 361 & 113 & 117 & 194 & 238 & 204 \\
\hline $45-64$ & 202 & 135 & 257 & 64 & 48 & 107 & 169 & 119 \\
\hline $65-84$ & 152 & 244 & 421 & 83 & 84 & 75 & 142 & 131 \\
\hline $85+$ & 127 & 580 & - & 75 & 381 & 182 & 103 & 143 \\
\hline
\end{tabular}

ICD10 C33-34

Source: The cancer registers in the Nordic countries; GL, Danish Cancer Society

Table 3.2.7 New cases of cancer of the testis per 1000000 men

\begin{tabular}{|c|c|c|c|c|c|c|c|c|}
\hline & Denmark & $\begin{array}{l}\text { Faroe } \\
\text { Islands }\end{array}$ & $\begin{array}{l}\text { Green- } \\
\text { land }\end{array}$ & Finland & Åland & Iceland & Norway & Sweden \\
\hline & 2014 & $2010-14$ & $2010-14$ & 2014 & $2010-14$ & $2010-14$ & 2014 & 2014 \\
\hline \multicolumn{9}{|l|}{ Age } \\
\hline $0-24$ & 45 & - & 55 & 35 & 49 & 31 & 69 & 40 \\
\hline $25-44$ & 212 & 296 & - & 172 & 56 & 107 & 68 & 175 \\
\hline $45-64$ & 101 & 62 & 23 & 30 & 101 & 45 & 39 & 55 \\
\hline $65-84$ & 25 & 61 & - & 9 & 101 & 35 & 12 & 23 \\
\hline $85+$ & - & - & - & 53 & - & - & - & - \\
\hline
\end{tabular}

ICD10 C62

Source: The cancer registers in the Nordic countries; GL, Danish Cancer Society 
Table 3.2.8 New cases of melanoma of the skin per 1000000 inhabitants

\begin{tabular}{lrrrrrrrr}
\hline & Denmark & $\begin{array}{c}\text { Faroe } \\
\text { Islands }\end{array}$ & Greenland & Finland & Åland & Iceland & Norway & Sweden \\
& 2014 & $2010-14$ & $2010-14$ & 2014 & $2010-14$ & $2010-14$ & 2014 & 2014 \\
\hline Men, age & & & & & & & & \\
$0-24$ & 10 & - & 18 & 8 & - & 7 & 10 & 10 \\
$25-44$ & 174 & 33 & - & 92 & 279 & 63 & 149 & 140 \\
$45-64$ & 433 & 124 & 68 & 341 & 456 & 152 & 500 & 475 \\
$65-84$ & 1144 & 305 & 288 & 758 & 872 & 421 & 1460 & 1215 \\
$85+$ & 1289 & - & - & 1117 & 850 & 521 & 2068 & 1706 \\
Women, age & & & & & & & & \\
$0-24$ & 52 & - & 38 & 22 & - & 40 & 12 & 22 \\
$25-44$ & 407 & 188 & 83 & 160 & 233 & 138 & 210 & 267 \\
$45-64$ & 528 & 68 & 57 & 294 & 819 & 265 & 561 & 521 \\
$65-84$ & 808 & 61 & - & 532 & 489 & 268 & 1027 & 770 \\
$85+$ & 763 & 580 & - & 724 & 761 & 121 & 1202 & 993 \\
\hline
\end{tabular}

ICD10 C43

Source: The cancer registers in the Nordic countries; GL, Danish Cancer Society

Table 3.2.9 National cancer screening programmes 1 January, 2016

\begin{tabular}{|c|c|c|c|c|c|c|}
\hline & Denmark & Greenland & Finland & Iceland & Norway & Sweden \\
\hline $\begin{array}{l}\text { Cancer of the } \\
\text { cervix uteri, } \\
\text { Women }\end{array}$ & $\begin{array}{l}\text { Yes, } 23-49 \\
\text { years every } 3 \\
\text { years. } 50-65 \\
\text { years every } 5 \\
\text { years }\end{array}$ & Yes & $\begin{array}{l}\text { Yes, 30-60 } \\
\text { years every } \\
\text { five years }\end{array}$ & $\begin{array}{l}\text { Yes, 23-65 } \\
\text { years, every } \\
3 \text { year }\end{array}$ & $\begin{array}{l}\text { Yes, 29-69 } \\
\text { years nation- } \\
\text { ally }\end{array}$ & $\begin{array}{l}\text { Yes, 23-64 } \\
\text { years }\end{array}$ \\
\hline $\begin{array}{l}\text { Breast cancer, } \\
\text { Women }\end{array}$ & $\begin{array}{l}\text { Yes, 50-69 } \\
\text { years every } \\
2 \text { years }\end{array}$ & No & $\begin{array}{l}\text { Yes, } \\
50-69 \text { years } \\
\text { every } 20-26 \\
\text { months }\end{array}$ & $\begin{array}{l}\text { Yes, 40-69 } \\
\text { years every } 2 \\
\text { years }\end{array}$ & $\begin{array}{l}\text { Yes, } 50-69 \\
\text { years na- } \\
\text { tionally }\end{array}$ & $\begin{array}{l}\text { Yes, 40-74 } \\
\text { years }\end{array}$ \\
\hline $\begin{array}{l}\text { Cancer of the } \\
\text { colon and rectum, } \\
\text { men and women }\end{array}$ & $\begin{array}{l}\text { Yes, Start- } \\
\text { ing 2014- } \\
2017.50- \\
74 \text { years }\end{array}$ & No & $\begin{array}{l}\text { Pilot project } \\
\text { (2004-) 60-69 } \\
\text { years region- } \\
\text { ally }\end{array}$ & No & $\begin{array}{l}\text { Pilot project } \\
(2012-) \text { 50-74 } \\
\text { years } \\
\text { regionally }\end{array}$ & $\begin{array}{l}\text { Yes, } \\
60-74 \\
\text { years }\end{array}$ \\
\hline $\begin{array}{l}\text { Cancer of the } \\
\text { testis, men }\end{array}$ & No & No & No & No & No & No \\
\hline Lung cancer & No & No & No & No & No & No \\
\hline
\end{tabular}


Figure 3.2.1 New cases of cancer, age-standardized rates per 1000000 inhabitants, 2000-2014
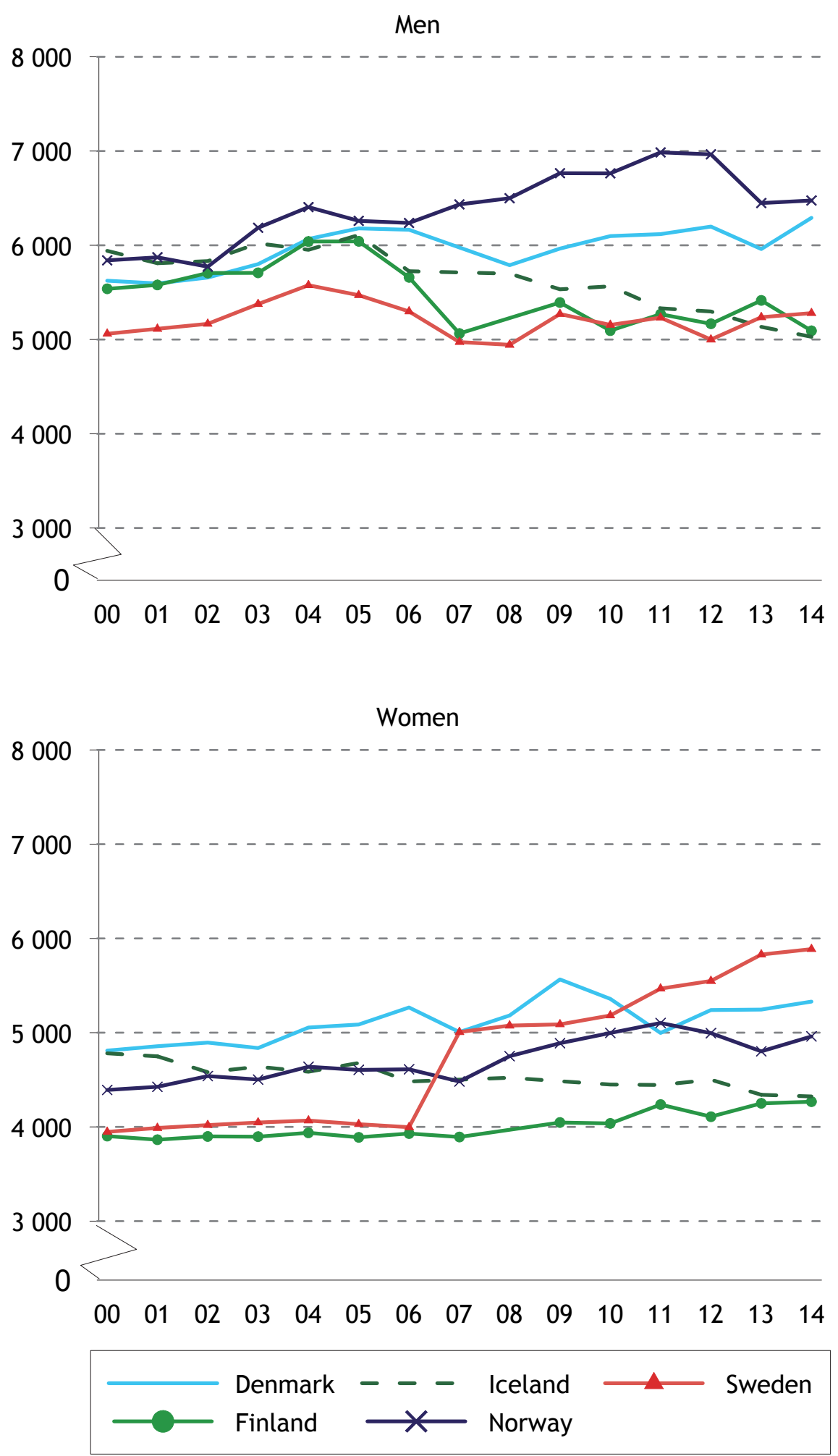

Age-standardized by the Nordic population 2000

The figures for Iceland are 5-year averages

Source: The cancer registers in the Nordic countries 
Morbidity, Medical Treatment, Accidents and Pharmaceutical products

Figure 3.2.2 New cases of prostate cancer (men), age-standardized rates per 1000000 inhabitants 2000-2014

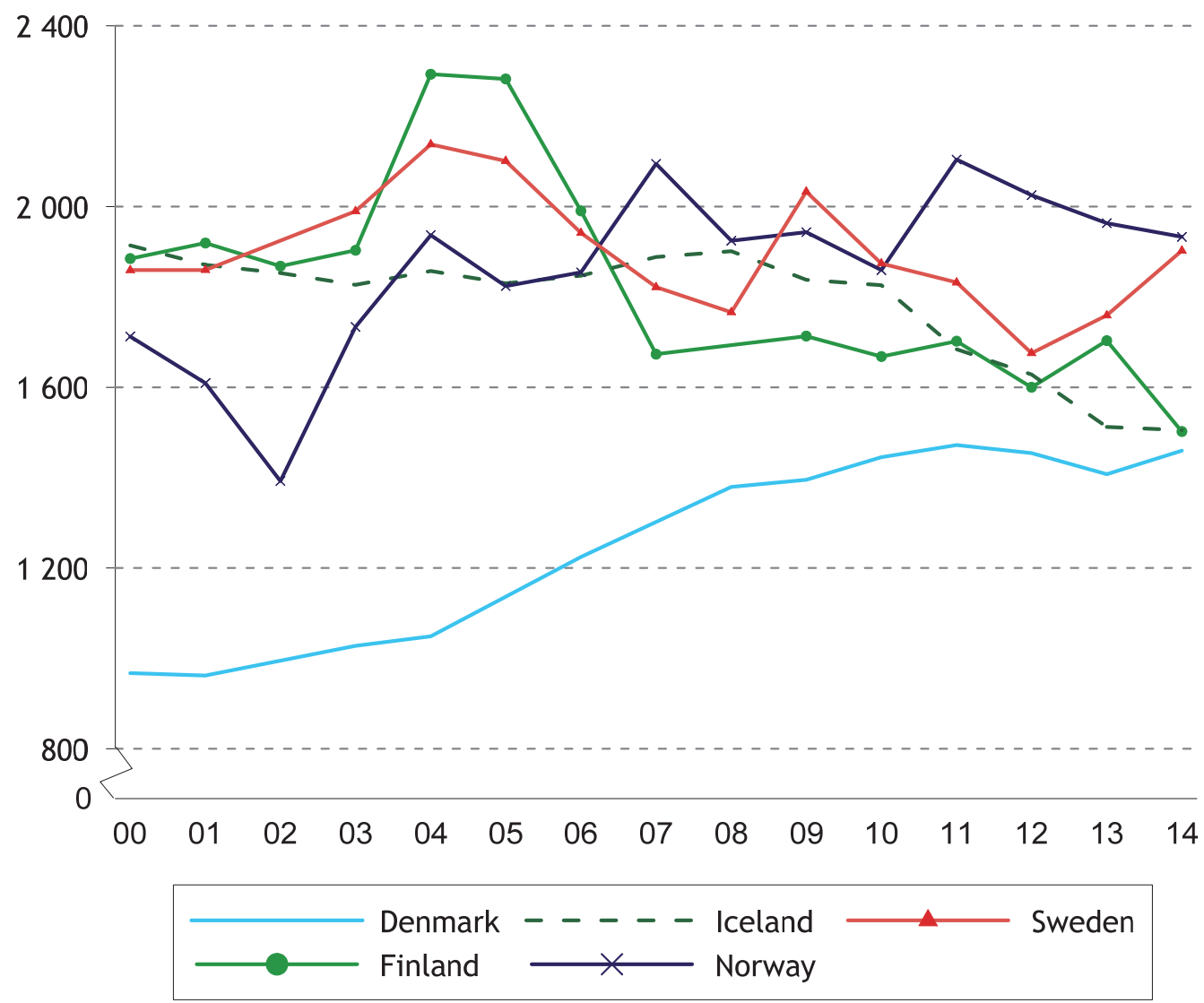

Age-standardized by the Nordic population 2000

The figures for Iceland are 5-year averages

Source: The cancer registers in the Nordic countries 
Figure 3.2.3 New cases of breast cancer (women), age-standardized rates per 1000000 inhabitants, 2000-2014

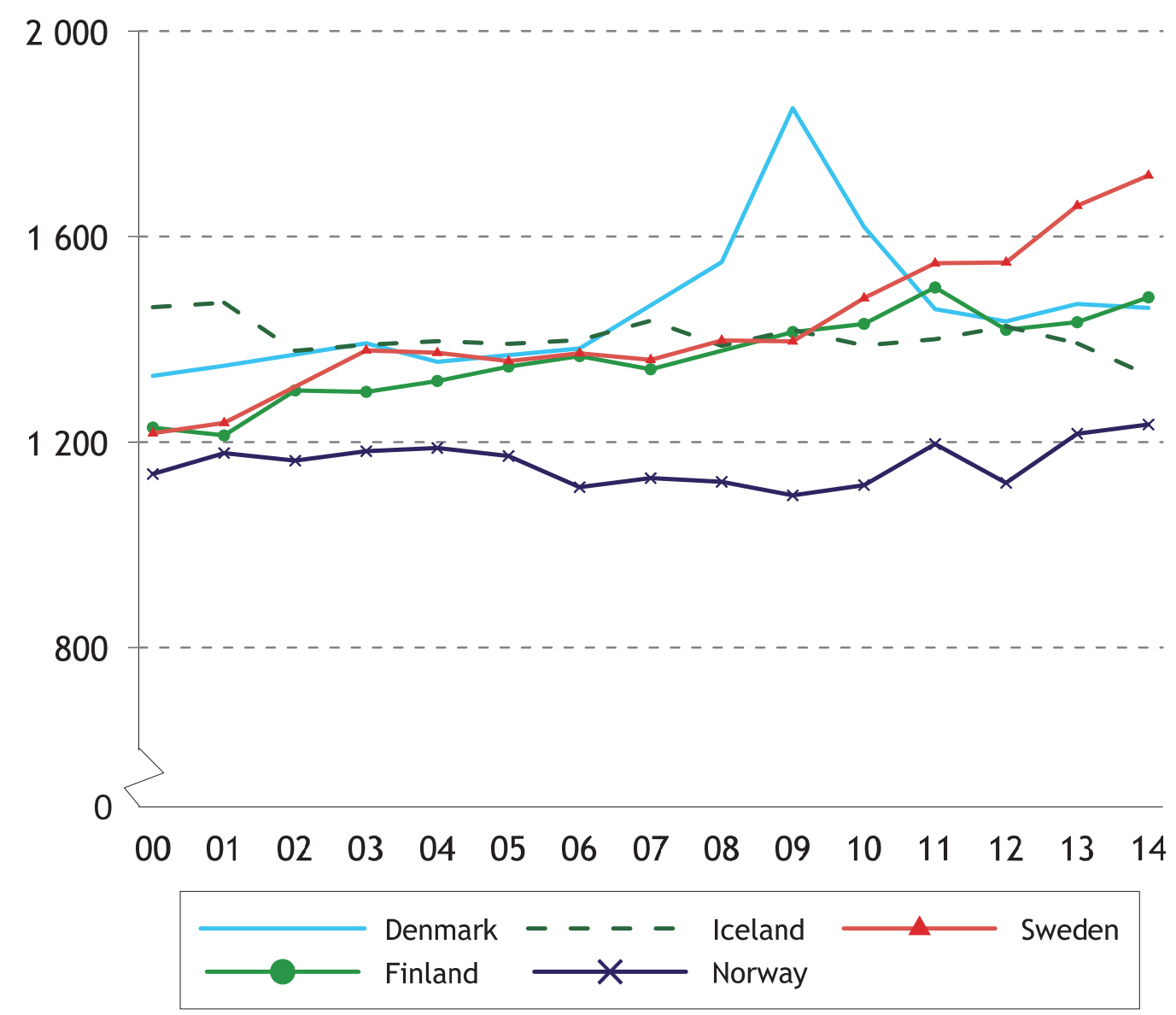

Age-standardized by the Nordic population 2000

The figures for Iceland are 5-year averages

Source: The cancer registers in the Nordic countries

The reason for the very large fluctuation in Figure 3.2.3 for Denmark is because screening for breast cancer became nationwide at the end of 2007. 
Figure 3.2.4 New cases of lung cancer, age-standardized rates per 1000000 inhabitants, 2000-2014
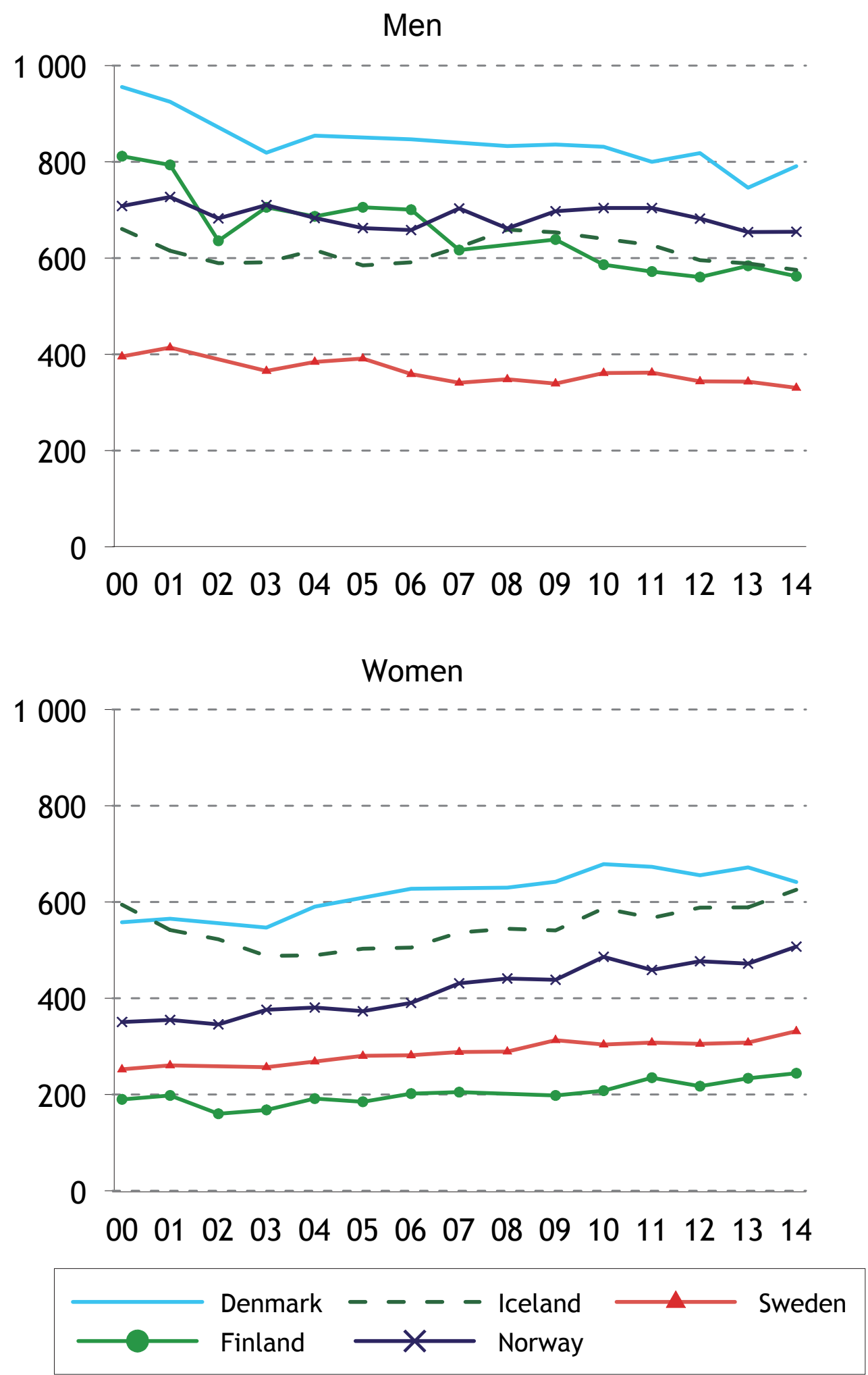

Age-standardized by the Nordic population 2000

The figures for Iceland are 5-year averages

Source: The cancer registers in the Nordic countries; GL, Danish Cancer Society 


\subsection{Immunization schedules}

All the Nordic countries have recommended immunization programmes with some differences in vaccination against tuberculosis and whooping cough, and the choice of vaccines against measles and rubella.

Collection of data on immunization varies a lot from country to country, and none of the countries, except Finland and Norway, have immunization registers covering the country as a whole.

Table 3.3.1 Recommended immunization schedules per 1 January $2016^{1}$

\begin{tabular}{|c|c|c|c|c|c|c|}
\hline & Denmark & Greenland & Finland & Iceland & Norway & Sweden \\
\hline Pneumococcus & $\begin{array}{l}3,5 \text { and } 12 \\
\text { months }\end{array}$ & $\begin{array}{l}3,5 \text { and } 12 \\
\text { months }\end{array}$ & $\begin{array}{l}3,5 \text { and } 12 \\
\text { months }+ \text { risk } \\
\text { group children } \\
\text { under } 5 \text { years }\end{array}$ & $\begin{array}{l}3,5,12 \\
\text { months; >60 } \\
\text { years and risk } \\
\text { groups }\end{array}$ & $\begin{array}{l}3,5 \text { and } 12 \\
\text { months, } \\
65+\text { years }\end{array}$ & .. \\
\hline BCG & - & At birth & $\begin{array}{l}\text { Only for risk } \\
\text { group children } \\
\text { under } 7 \text { years }\end{array}$ & - & Risk groups & Risk groups \\
\hline Pertussis & $\begin{array}{l}3,5 \text { and } 12 \\
\text { months } \\
\text { and } 5 \text { years }\end{array}$ & $\begin{array}{l}3,5 \text { and } 12 \\
\text { months } \\
\text { and } 5 \text { years }\end{array}$ & $\begin{array}{l}3,5 \text { and } 12 \\
\text { months, } \\
4 \text { and } 14-15 \\
\text { years }\end{array}$ & $\begin{array}{l}3,5,12 \\
\text { months, } \\
4 \text { and } 14 \text { years }\end{array}$ & $\begin{array}{l}3,5 \text { and } 12 \\
\text { months, } 7-8 \\
\text { years }\end{array}$ & $\begin{array}{l}3,5 \text { and } 12 \\
\text { months, } 5-6 \\
\text { and } 14-16 \\
\text { years }\end{array}$ \\
\hline Tetanus & $\begin{array}{l}3,5 \text { and } 12 \\
\text { months } \\
\text { and } 5 \text { years }\end{array}$ & $\begin{array}{l}3,5 \text { and } 12 \\
\text { months } \\
\text { and } 5 \text { years }\end{array}$ & $\begin{array}{l}3,5 \text { and } 12 \\
\text { months, } \\
4 \text { and } 14-15 \\
\text { years }\end{array}$ & $\begin{array}{l}3,5,12 \\
\text { months, } \\
4 \text { and } 14 \text { years, }\end{array}$ & $\begin{array}{l}3,5 \text { and } 12 \\
\text { months, } 7-8 \\
\text { years } 15-16 \\
\text { years }\end{array}$ & $\begin{array}{l}3,5 \text { and } 12 \\
\text { months, } 5-6 \\
\text { and } 14-16 \\
\text { years }\end{array}$ \\
\hline Diphtheria & $\begin{array}{l}3,5 \text { and } 12 \\
\text { months } \\
\text { and } 5 \text { years }\end{array}$ & $\begin{array}{l}3,5 \text { and } 12 \\
\text { months } \\
\text { and } 5 \text { years }\end{array}$ & $\begin{array}{l}3,5 \text { and } 12 \\
\text { months, } \\
4 \text { and } 14-15 \\
\text { years }\end{array}$ & $\begin{array}{l}3,5,12 \\
\text { months, } \\
4 \text { and } 14 \text { years }\end{array}$ & $\begin{array}{l}3,5 \text { and } 12 \\
\text { months, } 7-8 \\
\text { years } 15-16 \\
\text { years }\end{array}$ & $\begin{array}{l}3,5 \text { and } 12 \\
\text { months, } 5-6 \\
\text { and } 14-16 \\
\text { years }\end{array}$ \\
\hline Polio & $\begin{array}{l}\text { IPV: } 3,5,12 \\
\text { months and } 5 \\
\text { years }\end{array}$ & $\begin{array}{l}\text { IPV: } 3,5,12 \\
\text { months and } 5 \\
\text { years }\end{array}$ & $\begin{array}{l}\text { IPV: } 3,5 \text { and } \\
12 \text { months and } \\
4 \text { years }\end{array}$ & $\begin{array}{l}\text { IPV: } 3,5,12 \\
\text { months } \\
\text { and } 14 \text { years }\end{array}$ & $\begin{array}{l}\text { IPV: } 3,5 \text { and } \\
12 \text { months, } 7-8 \\
\text { years } 15-16 \\
\text { years }\end{array}$ & $\begin{array}{l}\text { IPV: } 3,5 \text { and } \\
12 \text { months, } 5-6 \\
\text { years }\end{array}$ \\
\hline $\begin{array}{l}\text { Measles, } \\
\text { Mumps, } \\
\text { Rubella }\end{array}$ & $\begin{array}{l}15 \text { months, } 4 \\
\text { years }\end{array}$ & $\begin{array}{l}15 \text { months, } 4 \\
\text { years }\end{array}$ & $\begin{array}{l}12-18 \text { months } \\
\text { and } 6 \text { years }\end{array}$ & $\begin{array}{l}18 \text { months and } \\
12 \text { years }\end{array}$ & $\begin{array}{l}15 \text { months and } \\
11-12 \text { years }\end{array}$ & $\begin{array}{l}18 \text { months } \\
\text { and } 6-8 \text { years }\end{array}$ \\
\hline Rubella, only & Fertile women & Fertile women & - & - & Fertile women & $\begin{array}{l}\text { A vaccine for } \\
\text { children is cur- } \\
\text { rently availa- } \\
\text { ble and is offe- } \\
\text { red in some } \\
\text { counties, but is } \\
\text { not included in } \\
\text { the Swedish } \\
\text { immunization } \\
\text { schedule for } \\
\text { children }\end{array}$ \\
\hline $\begin{array}{l}\text { Haemophilus } \\
\text { influenza b }\end{array}$ & $\begin{array}{l}3,5 \text { and } 12 \\
\text { months }\end{array}$ & $\begin{array}{l}3,5 \text { and } 12 \\
\text { months }\end{array}$ & $\begin{array}{l}3,5 \text { and } 12 \\
\text { months }\end{array}$ & $\begin{array}{l}3,5 \text { and } 12 \\
\text { months }\end{array}$ & $\begin{array}{l}3,5 \text { and } 12 \\
\text { months }\end{array}$ & $\begin{array}{l}3,5 \text { and } 12 \\
\text { months }\end{array}$ \\
\hline Rotavirus & & & $\begin{array}{l}2,3 \text { and } 5 \\
\text { months }\end{array}$ & & & \\
\hline HPV & Girls: 12 years & $\begin{array}{l}\text { Girls by their } \\
12 \text { th year, } 2 \\
\text { immunizations }\end{array}$ & $\begin{array}{l}\text { Girls: } 12-13 \\
\text { years }\end{array}$ & Girls: 12 years & $\begin{array}{l}12-13 \text { years } \\
\text { (girls only) }\end{array}$ & $\begin{array}{l}3 \text { immuniza- } \\
\text { tions for girls } \\
10-12 \text { years }\end{array}$ \\
\hline
\end{tabular}

Continues 
Table 3.3.1 Recommended immunization schedules per 1 January $2016^{1}$, continued

\begin{tabular}{|c|c|c|c|c|c|c|}
\hline & Denmark & Greenland & Finland & Iceland & Norway & Sweden \\
\hline $\begin{array}{l}\text { Meningococ- } \\
\text { cal disease } \\
\text { gr. C }\end{array}$ & - & - & - & $\begin{array}{l}6 \text { and } 8 \\
\text { months }\end{array}$ & - & $\begin{array}{l}\text { The vaccine } \\
\text { is given pri- } \\
\text { marily to } \\
\text { people who } \\
\text { plan to travel } \\
\text { to areas with } \\
\text { high morbidi- } \\
\text { ty, an epi- } \\
\text { demic, or an } \\
\text { outbreak }\end{array}$ \\
\hline Hepatitis b & $\begin{array}{l}\text { Risk groups } \\
\text { only }\end{array}$ & $\begin{array}{l}\text { At birth, 3, } 5 \\
\text { and } 12 \\
\text { months }\end{array}$ & $\begin{array}{l}\text { Risk groups } \\
\text { only }\end{array}$ & $\begin{array}{l}\text { Risk groups } \\
\text { only }\end{array}$ & .. & .. \\
\hline Influenza 65+ & $\begin{array}{l}65+\text { and risk } \\
\text { groups }\end{array}$ & $\begin{array}{l}65+\text { and risk } \\
\text { groups }\end{array}$ & $\begin{array}{l}6-35 \text { months, } \\
65+\text { years and } \\
\text { risk groups }\end{array}$ & $\begin{array}{l}>60 \text { years and } \\
\text { risk groups }\end{array}$ & $\begin{array}{l}65+\text { and risk } \\
\text { groups }\end{array}$ & $\begin{array}{l}65+\text { and risk } \\
\text { groups }\end{array}$ \\
\hline
\end{tabular}

1 Basically, the Faroe Islands and Åland have the same immunization schedules as Denmark and Finland respectively. However, the Faroe Islands give Influenza vaccination for age groups 67+. In Åland TBE is included for children over 4 years

Source: WHO/EPID, DK, the Danish Health Data Authority; GL, the Chief Medical Officer; FI, THL; IS, Directorate of Health; NO, Norwegian Institute of Public Health; SV, National Board of Health and Welfare

Table 3.3.2 Children under the age of two immunized according to recommended immunization schedules and elderly people vaccinated against influenza (per cent), 2014

\begin{tabular}{|c|c|c|c|c|c|c|}
\hline & Denmark $^{1}$ & $\begin{array}{c}\text { Faroe } \\
\text { Islands }{ }^{2}\end{array}$ & Finland $^{3,4}$ & Iceland $^{5}$ & Norway $^{6}$ & Sweden \\
\hline Pertussis & 90 & 94 & 98 & 92 & 94 & 98 \\
\hline Tetanus & 92 & 94 & 98 & 92 & 94 & 98 \\
\hline Diphtheria & 92 & 94 & 98 & 92 & 94 & 98 \\
\hline Polio & 92 & 94 & 98 & 92 & 94 & 98 \\
\hline Rubella ${ }^{7}$ & 88 & 90 & 95 & 93 & 93 & 98 \\
\hline Measles ${ }^{7}$ & 88 & 90 & 95 & 93 & 93 & 98 \\
\hline Influenza $65+$, in & & 40 & 40 & 41 & & 50 \\
\hline
\end{tabular}

1 Based on the immunization at 3 and 5 months

$267+$

3 Birth cohort 2013, based on National Vaccination Register (NVR). Due to data deficiencies in the register, coverage for at least one dose is reported

4 Rubella and measles: 12-18-month dose coverage from NVR. Based on earlier validation study, MMR coverage in the register is currently underestimated by $3 \%$

5 The number of persons vaccinated against pertussis, tetanus, diphtheria and polio is based on a birth cohort 2013, which received three doses of vaccine. The number of vaccinations against measles is based on a birth cohort 2012, which received one dose. For influenza 60+ the number is based on the number of persons vaccinated during the 4th quartile of 2014 and 1st quartile of 2015

6 The data is underestimated due to a low level of reporting in some municipalities

7 Sweden, MPR (Measles, Mumps, Rubella)

Source: WHO/EPI; DK, the Danish Health Data Authority; FO, Ministry of Health Affairs; FI, THL; IS, Directorate of Health; NO, Norwegian Institute of Public Health; SV, Public Health Agency of Sweden 


\subsection{Discharges, bed days, average length of stay and patients treated}

\section{Outline of this section}

In this section, diagnosis-related data on hospital use are presented according to the main diagnosis that has been registered for each hospital stay in the national patient registers of the Nordic countries. The presentation of diagnoses is more detailed than in NOMESCO publications from before 2010. It is now based on the new list of diagnoses developed by the EU Hospital Data Project. This list has been adopted by WHO as the International Shortlist for Hospital Morbidity Tabulation (ISHMT). It is also used by Eurostat, OECD and the WHO Regional Office for Europe.

The ISHMT list (see link ISHMT list of diagnoses) comprises 149 groups. Thus, it is relatively long for a traditional table presentation. Therefore, in this section, as a trial, we use an abbreviated list with selected groups from the full ISHMT list, among them the ICD-10 chapter-level groups that until now have been the principal grouping of diagnoses in the summary tables. Now 36 selected groups that are subgroups of the ICD-10 chapters have been added. Several principles have guided the choice of these groups. They are selected mainly because they are relatively common and/or of special interest for inter-Nordic comparison, e.g. because of new treatment possibilities. Some possible groups were not selected because hospital activities in those groups are reflected better in the statistics on procedures (cf. Section 3.5).

The presentation of the diagnosis-related statistics starts with tables of the total number of discharges (Table 3.4.1) and bed days (Table 3.4.2) per 100000 inhabitants. Besides the tables for both genders, separate tables for men and women are now included. This makes it possible to compare the two genders. However, age standardized tables for discharges and surgery procedures are not included (Section 3.5).

While discharge rates illustrate how common certain groups of diagnoses are as the reason for admission to hospital, bed-day rates give a better illustration of the load these diagnoses have on hospitals. The average length of stay for in-patients by diagnosis is shown in a third set of tables (Table 3.4.3). This is followed by figures that show the development over time of hospital use for three ICD chapters.

The section is concluded with ten detailed tables showing not only age distribution but also the relationship between number of discharges and number of patients treated for certain diagnosis groups. Since the patient registers make it possible to link successive hospital spells with the same main diagnosis, it is possible to calculate, on a national level, the total number of people who have been treated in a year.

\section{Quality and limitations of data}

The quality of the data in the patient registers, such as representativeness, completeness and reliability, is important for these statistics.

Nordic hospital data have a high degree of coverage.

In order to make the statistics as comparable as possible, the data presented in this section are from somatic hospital departments (wards) in general hospitals and specialized somatic wards. Still, it is not possible to get completely comparable sets 
of hospital data. In Norway, discharges are not related to hospital departments (wards) but only to the hospital as a whole, which means that discharge rates are slightly underestimated compared to the other countries.

This does not influence the bed-day rates, however. Furthermore, the data are influenced by the fact that some types of treatment for the Faroe Islands are provided in Denmark, and for Åland in Sweden.

The diagnosis-related statistics presented in this report are based on the main diagnosis for each hospital stay. The main diagnosis refers to the main condition treated or examined during each hospital stay. According to the ICD, it is defined as the condition, diagnosed at the end of the treatment period and primarily responsible for the patient's need for treatment or examination. This means that hospital statistics do not give a complete picture of the diseases treated in hospital, since the secondary diagnoses that have been attended to during a hospital stay do not show in the statistics. Hospital discharges, even when recalculated as number of patients treated, do not correspond to true incidence figures for the population, because not all cases are treated in hospitals. For certain diagnoses, incidence figures are available from other sources. This is the case for malignant neoplasms reported to the national cancer registers (cf. Section 3.2). Hospital data for cancer diagnoses are complementary to these, in the sense that they illustrate how cancer morbidity is reflected in the activity and workload of hospitals.

Comparisons between countries are also hampered by the fact that there are some differences in the way the WHO definition of main condition is interpreted in the Nordic countries. The introduction of Diagnosis Related Groups (DRG) has influenced the choice of main diagnosis in all the countries, but slightly differently.

There are also national differences in diagnostic tradition (as will be shown below) and differences in registration and coding of diagnoses that influence comparability.

Healthy new-born babies are counted differently in the Nordic countries. In the $I C D$, there is a category (Z38) and in the ISHMT list, there is a group for healthy newborn babies. In some of the countries, these babies are not registered as patients in their own right and thus they are not included in the patient registers. Therefore, healthy new-born babies are excluded from the tables in this section. 


\section{Comments to the tables}

The overall discharge rates (cf. Table 3.4.1.a) vary somewhat between the Nordic countries. Highest rates are found for Denmark and the lowest for Iceland with Finland, Norway and Sweden in-between. There are marked differences, however, in hospital use between the countries for certain groups of diseases and specific diagnoses, both measured as rate of discharges and as rate of bed-days.

In all countries, there are high discharge rates for diseases of the circulatory system (ICD, Chapter IX), injuries (Chapter XIX) and neoplasms (Chapter II). In Iceland, however, pregnancy and childbirth (Chapter XV) account for the highest discharge rate, and in Denmark discharges for factors influencing health status and contact with health services (Chapter XXI) is the most common of all ICD chapters.

For many diagnosis groups and for specific diagnoses, there is also great similarity in average length of stay. However, there are some greater differences between the countries, such as for mental and behavioural disorders with long stays for Finland, Denmark and Sweden. This reflects the fact that the somatic hospital data in these countries include some psychiatric patients. Long stays are also found for cerebrovascular diseases in the same countries, indicating the occurrence of some long-term care cases in short-term hospitals in these countries.

While some of the differences in hospital use may be due to slightly different disease patterns in the Nordic countries, it is obvious that many of the differences in the statistics are attributable to organizational differences in the hospital systems and to differences in the registration and coding of diagnoses in hospitals.

A clear example of this is the very high discharge rate for Denmark for Chapter XXI and especially for medical observation and evaluation for suspected diseases and conditions (code Z03). As can be seen from Table 3.4.1, there are large differences between the countries in this area. Apparently, cases with a suspected but not quite confirmed diagnosis are coded differently. While such a case may be coded as a symptom or as a definite disease in other countries, in Denmark they are often coded as an observation case (Z03). Other examples of differences in coding practice refer to the use in Denmark and Norway of a Chapter XXI code for rehabilitation cases (code Z50, not specified in the tables). In other countries, rehabilitation cases seem to a greater extent to be coded to the underlying disorder.

The trends illustrated in Figures 3.4.1 - 3 do not show big changes in discharge rates over the years (except for Åland, due to small populations). The other countries retain their relative positions in relation to each other over the period studied.

In Tables 3.4.4 - 3.4.13, the possibilities of linking successive hospital stays for the same main diagnosis and the same person have been used, thus calculating the number of actual persons being treated, in the following called 'patients treated'. The Nordic countries are among the few countries in the world that can do this on a national level. As an example, from Table 3.4.4 on lung cancer, it can be seen that for all countries and for both men and women the number of patients treated is about half the number of discharges.

It is also worth noting that the age-specific rates for patients treated for lung cancer are at the same level for both genders under the age of 65 ; men have higher rates only in the age group 65 and over. 
Morbidity, Medical Treatment, Accidents and Pharmaceutical products

The difference in the number of discharges and the number of patients treated varies by diagnosis. The difference is largest for chronic conditions such as chronic obstructive pulmonary disease (Table 3.4.8) and alcoholic liver disease (Table 3.4.10).

In all countries, the number of patients treated amounts to about 60 per cent of the number of discharges for these two diseases. For most of the other diagnoses presented in the detailed tables, the number of patients treated corresponds to 70 80 per cent of the number of discharges. 
Table 3.4.1.a Discharges from hospitals per 100000 inhabitants by main diagnosis, both genders

\begin{tabular}{|c|c|c|c|c|c|c|}
\hline ICD-10 code & Denmark & Finland & Åland & Iceland $^{1}$ & Norway & Sweden \\
\hline Main diagnosis & 2014 & 2014 & $2010-14$ & 2014 & 2014 & 2014 \\
\hline $\begin{array}{l}\text { I: Certain infectious and } \\
\text { parasitic diseases (A00-B99) }\end{array}$ & 838 & 544 & 541 & 154 & 432 & 497 \\
\hline II: Neoplasms (COO-D48) & 1700 & 1575 & 1109 & 981 & 1600 & 1209 \\
\hline $\begin{array}{l}\text { III: Diseases of the blood and } \\
\text { blood forming organs and certain } \\
\text { disorders involving the } \\
\text { immune mechanism (D50-D89) }\end{array}$ & 264 & 134 & 137 & 83 & 159 & 147 \\
\hline $\begin{array}{l}\text { IV: Endocrine, nutritional and } \\
\text { metabolic diseases (EOO-E90) }\end{array}$ & 753 & 297 & 219 & 207 & 346 & 361 \\
\hline $\begin{array}{l}\text { V: Mental and behavioural disorders } \\
\text { (FO0-F99) }\end{array}$ & 1185 & 846 & 536 & 197 & 295 & 1220 \\
\hline $\begin{array}{l}\text { VI: Diseases of the nervous system } \\
\text { (G00-G99) }\end{array}$ & 648 & 593 & 498 & 341 & 659 & 469 \\
\hline $\begin{array}{l}\text { VII: Diseases of the eye and adnexa } \\
(\text { HOO-H59) }\end{array}$ & 86 & 140 & 34 & 58 & 114 & 86 \\
\hline $\begin{array}{l}\text { VIII: Diseases of the ear and } \\
\text { mastoid process }(\mathrm{H} 60-\mathrm{H} 95)\end{array}$ & 114 & 70 & 130 & 32 & 83 & 88 \\
\hline $\begin{array}{l}\text { IX: Diseases of the circulatory system } \\
(100-199)\end{array}$ & 2543 & 2313 & 1999 & 1123 & 2257 & 2265 \\
\hline $\begin{array}{l}X: \text { Diseases of the respiratory system } \\
(J 00-J 99)\end{array}$ & 2122 & 1099 & 1183 & 580 & 1281 & 1068 \\
\hline $\begin{array}{l}\text { XI: Diseases of the digestive system } \\
\text { (K00-K93) }\end{array}$ & 1977 & 1255 & 1356 & 758 & 1311 & 1287 \\
\hline $\begin{array}{l}\text { XII: Diseases of the skin and } \\
\text { subcutaneous tissue (L00-L99) }\end{array}$ & 323 & 150 & 97 & 160 & 168 & 141 \\
\hline $\begin{array}{l}\text { XIII: Diseases of the musculoskeletal } \\
\text { system and connective tissue (M00-M99) }\end{array}$ & 1313 & 1077 & 1323 & 594 & 1156 & 968 \\
\hline $\begin{array}{l}\text { XIV: Diseases of the genitourinary } \\
\text { system (NOO-N99) }\end{array}$ & 1283 & 831 & 1029 & 546 & 966 & 803 \\
\hline $\begin{array}{l}X V \text { : Pregnancy, childbirth and } \\
\text { the puerperium (000-099) }\end{array}$ & 1327 & 1274 & 1174 & 1527 & 610 & 1428 \\
\hline $\begin{array}{l}\mathrm{XVI} \text { : Certain conditions originating in } \\
\text { the perinatal period }(P 00-P 96)\end{array}$ & 185 & 169 & 122 & 367 & 172 & 162 \\
\hline $\begin{array}{l}\text { XVII: Congenital malformations, } \\
\text { deformations and chromosomal ab- } \\
\text { normalities (Q00-Q99) }\end{array}$ & 176 & 136 & 52 & 147 & 143 & 104 \\
\hline $\begin{array}{l}\text { XVIII: Symptoms, signs and abnormal } \\
\text { clinical and laboratory findings, not } \\
\text { elsewhere classified (R00-R99) }\end{array}$ & 2915 & 953 & 1406 & 642 & 1413 & 1555 \\
\hline $\begin{array}{l}\text { XIX: Injury, poisoning and certain other } \\
\text { consequences of external causes (SOO-T98) }\end{array}$ & 2128 & 1527 & 1331 & 849 & 1788 & 1570 \\
\hline $\begin{array}{l}\text { XXI: Factors influencing health status and } \\
\text { contact with health services (ZOO-Z99) }\end{array}$ & 2837 & 220 & 848 & 806 & 1647 & 616 \\
\hline $\begin{array}{l}\text { All causes (except. } X X) \\
\text { (A00-Z99 excluding } V, W, X \text { and } Y \text { ) }\end{array}$ & 25716 & 15202 & 15123 & 10153 & 16602 & 17044 \\
\hline
\end{tabular}

1 Only discharges with a length of stay less than 90 days

Source: The national in-patient registers 
Table 3.4.1.b Discharges from hospitals per 100000 inhabitants by main diagnosis, men

\begin{tabular}{|c|c|c|c|c|c|c|}
\hline ICD-10 code & Denmark & Finland & Åland & Iceland ${ }^{1}$ & Norway & Sweden \\
\hline Main diagnosis & 2014 & 2014 & $2010-14$ & 2014 & 2014 & 2014 \\
\hline $\begin{array}{l}\text { I: Certain infectious and } \\
\text { parasitic diseases (A00-B99) }\end{array}$ & 910 & 567 & 572 & 144 & 450 & 522 \\
\hline II: Neoplasms (C00-D48) & 1665 & 1514 & 936 & 906 & 1578 & 1141 \\
\hline $\begin{array}{l}\text { III: Diseases of the blood and } \\
\text { blood forming organs and certain } \\
\text { disorders involving the } \\
\text { immune mechanism (D50-D89) }\end{array}$ & 257 & 124 & 143 & 81 & 140 & 129 \\
\hline $\begin{array}{l}\text { IV: Endocrine, nutritional and } \\
\text { metabolic diseases (E00-E90) }\end{array}$ & 653 & 255 & 192 & 120 & 277 & 303 \\
\hline $\begin{array}{l}\text { V: Mental and behavioural disorders } \\
\text { (F00-F99) }\end{array}$ & 1239 & 875 & 519 & 169 & 335 & 1319 \\
\hline $\begin{array}{l}\text { VI: Diseases of the nervous system } \\
\text { (G00-G99) }\end{array}$ & 664 & 590 & 445 & 342 & 669 & 469 \\
\hline $\begin{array}{l}\text { VII: Diseases of the eye and adnexa } \\
(\mathrm{H} 00-\mathrm{H} 59)\end{array}$ & 87 & 134 & 21 & 59 & 116 & 89 \\
\hline $\begin{array}{l}\text { VIII: Diseases of the ear and } \\
\text { mastoid process ( } \mathrm{H} 60-\mathrm{H} 95)\end{array}$ & 117 & 70 & 137 & 25 & 78 & 81 \\
\hline $\begin{array}{l}\text { IX: Diseases of the circulatory system } \\
(100-199)\end{array}$ & 3064 & 2592 & 2076 & 1388 & 2705 & 2599 \\
\hline $\begin{array}{l}\mathrm{X}: \text { Diseases of the respiratory system } \\
(\mathrm{J} 00-\mathrm{J} 99)\end{array}$ & 2242 & 1234 & 1354 & 560 & 1327 & 1088 \\
\hline $\begin{array}{l}\text { XI: Diseases of the digestive system } \\
\text { (K00-K93) }\end{array}$ & 1991 & 1338 & 1342 & 695 & 1295 & 1271 \\
\hline $\begin{array}{l}\text { XII: Diseases of the skin and } \\
\text { subcutaneous tissue (L00-L99) }\end{array}$ & 356 & 165 & 103 & 177 & 176 & 143 \\
\hline $\begin{array}{l}\text { XIII: Diseases of the musculoskeletal } \\
\text { system and connective tissue (M00- } \\
\text { M99) }\end{array}$ & 1202 & 916 & 1128 & 513 & 1015 & 853 \\
\hline $\begin{array}{l}\text { XIV: Diseases of the genitourinary } \\
\text { system (N00-N99) }\end{array}$ & 1106 & 689 & 623 & 379 & 904 & 776 \\
\hline $\begin{array}{l}X V \text { : Pregnancy, childbirth and } \\
\text { the puerperium (000-099) }\end{array}$ &.. &.. &.. & .. & .. & .. \\
\hline $\begin{array}{l}\mathrm{XVI} \text { : Certain conditions originating in } \\
\text { the perinatal period ( } \mathrm{P00}-\mathrm{P} 96)\end{array}$ & 216 & 191 & 134 & 423 & 188 & 180 \\
\hline $\begin{array}{l}\text { XVII: Congenital malformations, } \\
\text { deformations and chromosomal a } \\
\text { normalities (Q00-Q99) }\end{array}$ & 200 & 145 & 55 & 161 & 162 & 117 \\
\hline $\begin{array}{l}\text { XVIII: Symptoms, signs and abnormal } \\
\text { clinical and laboratory findings, not } \\
\text { elsewhere classified (R00-R99) }\end{array}$ & 2735 & 925 & 1249 & 539 & 1299 & 1471 \\
\hline $\begin{array}{l}\text { XIX: Injury, poisoning and certain } \\
\text { other consequences of external causes } \\
\text { (S00-T98) }\end{array}$ & 2119 & 1578 & 1361 & 819 & 1779 & 1501 \\
\hline $\begin{array}{l}\text { XXI: Factors influencing health status } \\
\text { and contact with health services (Z00- } \\
\text { Z99) }\end{array}$ & 2714 & 184 & 682 & 627 & 815 & 592 \\
\hline $\begin{array}{l}\text { All causes (except. } \mathrm{XX} \text { ) } \\
\text { (A00-Z99 excluding } \mathrm{V}, \mathrm{W}, \mathrm{X} \text { and } \mathrm{Y} \text { ) }\end{array}$ & 24572 & 14087 & 13071 & 8126 & 15308 & 15674 \\
\hline
\end{tabular}

1 Only discharges with a length of stay less than 90 days

Source: The national in-patient registers 
Table 3.4.1.c Discharges from hospitals per 100000 inhabitants by main diagnosis, women

\begin{tabular}{|c|c|c|c|c|c|c|}
\hline ICD-10 code & Denmark & Finland & Åland & Iceland $^{1}$ & Norway & Sweden \\
\hline Main diagnosis & 2014 & 2014 & $2010-14$ & 2014 & 2014 & 2014 \\
\hline $\begin{array}{l}\text { I: Certain infectious and } \\
\text { parasitic diseases (A00-B99) }\end{array}$ & 767 & 522 & 509 & 164 & 414 & 473 \\
\hline II: Neoplasms (C00-D48) & 1735 & 1633 & 1280 & 1057 & 1623 & 1278 \\
\hline $\begin{array}{l}\text { III: Diseases of the blood and } \\
\text { blood forming organs and certain } \\
\text { disorders involving the } \\
\text { immune mechanism (D50-D89) }\end{array}$ & 272 & 144 & 131 & 86 & 177 & 164 \\
\hline $\begin{array}{l}\text { IV: Endocrine, nutritional and } \\
\text { metabolic diseases (E00-E90) }\end{array}$ & 852 & 337 & 246 & 294 & 417 & 418 \\
\hline $\begin{array}{l}\text { V: Mental and behavioural disorders } \\
\text { (F00-F99) }\end{array}$ & 1132 & 818 & 552 & 224 & 255 & 1120 \\
\hline $\begin{array}{l}\text { VI: Diseases of the nervous system } \\
\text { (G00-G99) }\end{array}$ & 632 & 596 & 550 & 340 & 648 & 468 \\
\hline $\begin{array}{l}\text { VII: Diseases of the eye and adnexa } \\
(\mathrm{H} 00-\mathrm{H} 59)\end{array}$ & 86 & 146 & 46 & 57 & 111 & 83 \\
\hline $\begin{array}{l}\text { VIII: Diseases of the ear and } \\
\text { mastoid process }(\mathrm{H} 60-\mathrm{H} 95)\end{array}$ & 111 & 70 & 122 & 39 & 88 & 94 \\
\hline $\begin{array}{l}\text { IX: Diseases of the circulatory system } \\
(100-199)\end{array}$ & 2029 & 2044 & 1919 & 856 & 1805 & 1932 \\
\hline $\begin{array}{l}\mathrm{X} \text { : Diseases of the respiratory system } \\
(\mathrm{J} 00-\mathrm{J} 99)\end{array}$ & 2004 & 968 & 1010 & 601 & 1234 & 1048 \\
\hline $\begin{array}{l}\mathrm{XI}: \text { Diseases of the digestive system } \\
\text { (K00-K93) }\end{array}$ & 1963 & 1174 & 1367 & 822 & 1328 & 1302 \\
\hline $\begin{array}{l}\text { XII: Diseases of the skin and } \\
\text { subcutaneous tissue (L00-L99) }\end{array}$ & 291 & 136 & 90 & 142 & 160 & 139 \\
\hline $\begin{array}{l}\text { XIII: Diseases of the musculoskeletal } \\
\text { system and connective tissue (M00- } \\
\text { M99) }\end{array}$ & 1423 & 1233 & 1514 & 676 & 1299 & 1083 \\
\hline $\begin{array}{l}\text { XIV: Diseases of the genitourinary } \\
\text { system (N00-N99) }\end{array}$ & 1456 & 969 & 1432 & 714 & 1029 & 830 \\
\hline $\begin{array}{l}\text { XV: Pregnancy, childbirth and } \\
\text { the puerperium (000-099) }\end{array}$ & 2635 & 2507 & 2338 & 3065 & 1228 & 2854 \\
\hline $\begin{array}{l}\mathrm{XVI} \text { : Certain conditions originating in } \\
\text { the perinatal period ( } \mathrm{POO}-\mathrm{P} 96)\end{array}$ & 154 & 148 & 109 & 311 & 156 & 144 \\
\hline $\begin{array}{l}\text { XVII: Congenital malformations, } \\
\text { deformations and chromosomal ab- } \\
\text { normalities (Q00-Q99) }\end{array}$ & 152 & 127 & 49 & 134 & 123 & 91 \\
\hline $\begin{array}{l}\text { XVIII: Symptoms, signs and abnormal } \\
\text { clinical and laboratory findings, not } \\
\text { elsewhere classified (R00-R99) }\end{array}$ & 3092 & 979 & 1559 & 746 & 1528 & 1639 \\
\hline $\begin{array}{l}\text { XIX: Injury, poisoning and certain } \\
\text { other consequences of external causes } \\
\text { (S00-T98) }\end{array}$ & 2136 & 1477 & 1300 & 879 & 1797 & 1639 \\
\hline $\begin{array}{l}\text { XXI: Factors influencing health status } \\
\text { and contact with health services (Z00- } \\
\text { Z99) }\end{array}$ & 2959 & 254 & 1012 & 986 & 2489 & 640 \\
\hline $\begin{array}{l}\text { All causes (except. XX) } \\
\text { (A00-Z99 excluding } \mathrm{V}, \mathrm{W}, \mathrm{X} \text { and } \mathrm{Y} \text { ) }\end{array}$ & 26843 & 16282 & 17135 & 12194 & 17911 & 18412 \\
\hline
\end{tabular}

1 Only discharges with a length of stay less than 90 days

Source: The national in-patient registers 
Table 3.4.2.a Bed days in hospitals per 100000 inhabitants by main diagnosis, both genders

\begin{tabular}{|c|c|c|c|c|c|c|}
\hline ICD-10 code & Denmark & Finland & Åland & Iceland $^{1}$ & Norway & Sweden \\
\hline Main diagnosis & 2014 & 2014 & $2010-14$ & 2014 & 2014 & 2014 \\
\hline $\begin{array}{l}\text { I: Certain infectious and } \\
\text { parasitic diseases (A00-B99) }\end{array}$ & 3494 & 3803 & 3683 & 907 & 2266 & 2713 \\
\hline II: Neoplasms (COO-D48) & 6719 & 9540 & 10450 & 7988 & 8699 & 8356 \\
\hline $\begin{array}{l}\text { III: Diseases of the blood and } \\
\text { blood forming organs and certain } \\
\text { disorders involving the } \\
\text { immune mechanism (D50-D89) }\end{array}$ & 682 & 637 & 811 & 544 & 568 & 635 \\
\hline $\begin{array}{l}\text { IV: Endocrine, nutritional and } \\
\text { metabolic diseases (EOO-E90) }\end{array}$ & 2395 & 1806 & 1659 & 1388 & 1212 & 1602 \\
\hline $\begin{array}{l}\text { V: Mental and behavioural disorders } \\
\text { (FOO-F99) }\end{array}$ & 16450 & 25391 & 7810 & 2982 & 628 & 15144 \\
\hline $\begin{array}{l}\text { VI: Diseases of the nervous system } \\
\text { (G00-G99) }\end{array}$ & 2669 & 6443 & 18489 & 2954 & 2213 & 2343 \\
\hline $\begin{array}{l}\text { VII: Diseases of the eye and adnexa } \\
\text { (HOO-H59) }\end{array}$ & 160 & 387 & 110 & 207 & 336 & 203 \\
\hline $\begin{array}{l}\text { VIII: Diseases of the ear and } \\
\text { mastoid process }(\mathrm{H} 6 \mathrm{O}-\mathrm{H} 95)\end{array}$ & 192 & 220 & 322 & 149 & 159 & 196 \\
\hline $\begin{array}{l}\text { IX: Diseases of the circulatory system } \\
(100-199)\end{array}$ & 8838 & 18073 & 16837 & 9864 & 9268 & 12386 \\
\hline $\begin{array}{l}X: \text { Diseases of the respiratory system } \\
(J 00-J 99)\end{array}$ & 7791 & 6901 & 7345 & 4554 & 6879 & 5607 \\
\hline $\begin{array}{l}\mathrm{XI}: \text { Diseases of the digestive system } \\
(\text { KOO-K93) }\end{array}$ & 6389 & 5970 & 6955 & 3951 & 5207 & 5491 \\
\hline $\begin{array}{l}\text { XII: Diseases of the skin and } \\
\text { subcutaneous tissue (L00-L99) }\end{array}$ & 975 & 1070 & 746 & 1017 & 962 & 846 \\
\hline $\begin{array}{l}\text { XIII: Diseases of the musculoskeletal } \\
\text { system and connective tissue (M00-M99) }\end{array}$ & 4042 & 5371 & 6139 & 3760 & 4537 & 4291 \\
\hline $\begin{array}{l}\text { XIV: Diseases of the genitourinary } \\
\text { system (N00-N99) }\end{array}$ & 3322 & 3879 & 4822 & 2195 & 3301 & 3338 \\
\hline $\begin{array}{l}X V \text { : Pregnancy, childbirth and } \\
\text { the puerperium (000-099) }\end{array}$ & 3187 & 5107 & 5506 & 3128 & 1983 & 3463 \\
\hline $\begin{array}{l}\mathrm{XVI} \text { : Certain conditions originating in } \\
\text { the perinatal period }(P 00-P 96)\end{array}$ & 1502 & 1408 & 1113 & 1774 & 1618 & 1562 \\
\hline $\begin{array}{l}\text { XVII: Congenital malformations, } \\
\text { deformations and chromosomal ab- } \\
\text { normalities (Q00-Q99) }\end{array}$ & 506 & 632 & 325 & 588 & 636 & 518 \\
\hline $\begin{array}{l}\text { XVIII: Symptoms, signs and abnormal } \\
\text { clinical and laboratory findings, not } \\
\text { elsewhere classified (RO0-R99) }\end{array}$ & 5347 & 3561 & 5305 & 2883 & 2447 & 3999 \\
\hline $\begin{array}{l}\text { XIX: Injury, poisoning and certain } \\
\text { other consequences of external causes } \\
\text { (SOO-T98) }\end{array}$ & 6617 & 10160 & 9131 & 6250 & 6890 & 7907 \\
\hline $\begin{array}{l}\text { XXI: Factors influencing health status } \\
\text { and contact with health services (Z00- } \\
\text { Z99) }\end{array}$ & 12410 & 1337 & 10486 & 5998 & 7310 & 2425 \\
\hline $\begin{array}{l}\text { All causes (except. } X X) \\
\text { (A00-Z99 excluding } V, W, X \text { and } Y \text { ) }\end{array}$ & 95654 & 111696 & 118045 & 63080 & 67118 & 91427 \\
\hline
\end{tabular}

1 Only discharges with a length of stay less than 90 days

Source: The national in-patient registers 
Table 3.4.2.b Bed days in hospitals per 100000 inhabitants by main diagnosis, men

\begin{tabular}{|c|c|c|c|c|c|c|}
\hline ICD-10 code & Denmark & Finland & Åland & Iceland $^{1}$ & Norway & Sweden \\
\hline Main diagnosis & 2014 & 2014 & $2010-14$ & 2014 & 2014 & 2014 \\
\hline $\begin{array}{l}\text { I: Certain infectious and } \\
\text { parasitic diseases (A00-B99) }\end{array}$ & 3878 & 4005 & 4182 & 925 & 2426 & 2831 \\
\hline II: Neoplasms (C00-D48) & 7112 & 9321 & 9648 & 7329 & 9069 & 8242 \\
\hline $\begin{array}{l}\text { III: Diseases of the blood and } \\
\text { blood forming organs and certain } \\
\text { disorders involving the } \\
\text { immune mechanism (D50-D89) }\end{array}$ & 661 & 566 & 1001 & 514 & 547 & 582 \\
\hline $\begin{array}{l}\text { IV: Endocrine, nutritional and } \\
\text { metabolic diseases (E00-E90) }\end{array}$ & 2243 & 1678 & 1347 & 948 & 1060 & 1495 \\
\hline $\begin{array}{l}\text { V: Mental and behavioural disorders } \\
\text { (F00-F99) }\end{array}$ & 15775 & 23530 & 6322 & 2687 & 616 & 15557 \\
\hline $\begin{array}{l}\text { VI: Diseases of the nervous system } \\
\text { (G00-G99) }\end{array}$ & 2702 & 5340 & 10360 & 2972 & 2178 & 2417 \\
\hline $\begin{array}{l}\text { VII: Diseases of the eye and adnexa } \\
\text { (H00-H59) }\end{array}$ & 165 & 362 & 72 & 173 & 335 & 198 \\
\hline $\begin{array}{l}\text { VIII: Diseases of the ear and mastoid } \\
\text { process (H60-H95) }\end{array}$ & 207 & 228 & 330 & 83 & 145 & 177 \\
\hline $\begin{array}{l}\text { IX: Diseases of the circulatory system } \\
(100-199)\end{array}$ & 10612 & 17797 & 14383 & 11784 & 10931 & 13586 \\
\hline $\begin{array}{l}\text { X: Diseases of the respiratory system } \\
\text { (J00-J99) }\end{array}$ & 8280 & 7521 & 8568 & 4093 & 7147 & 5605 \\
\hline $\begin{array}{l}\text { XI: Diseases of the digestive system } \\
\text { (K00-K93) }\end{array}$ & 64 & 6424 & 7155 & 3588 & 5086 & 5317 \\
\hline $\begin{array}{l}\text { XII: Diseases of the skin and } \\
\text { subcutaneous tissue (LOO-L99) }\end{array}$ & 1103 & 1100 & 847 & 1062 & 984 & 842 \\
\hline $\begin{array}{l}\text { XIII: Diseases of the musculoskeletal } \\
\text { system and connective tissue (M00-M99) }\end{array}$ & 3469 & 4367 & 4678 & 2904 & 3963 & 3558 \\
\hline $\begin{array}{l}\text { XIV: Diseases of the genitourinary } \\
\text { system (NOO-N99) }\end{array}$ & 3117 & 3302 & 3460 & 1711 & 3324 & 3389 \\
\hline $\begin{array}{l}X V \text { : Pregnancy, childbirth and } \\
\text { the puerperium (000-099) }\end{array}$ & .. & .. &.. &.. &.. &.. \\
\hline $\begin{array}{l}\mathrm{XVI} \text { : Certain conditions originating in } \\
\text { the perinatal period (POO-P96) }\end{array}$ & 1654 & 1598 & 1269 & 1958 & 1775 & 1745 \\
\hline $\begin{array}{l}\text { XVII: Congenital malformations, } \\
\text { deformations and } \\
\text { chromosomal abnormalities (Q00-Q99) }\end{array}$ & 562 & 692 & 368 & 684 & 700 & 590 \\
\hline $\begin{array}{l}\text { XVIII: Symptoms, signs and abnormal } \\
\text { clinical and laboratory findings, not } \\
\text { elsewhere classified (R00-R99) }\end{array}$ & 5113 & 3373 & 4761 & 2449 & 2251 & 3749 \\
\hline $\begin{array}{l}\text { XIX: Injury, poisoning and certain } \\
\text { other consequences of external causes } \\
\text { (SOO-T98) }\end{array}$ & 6318 & 9894 & 10350 & 5917 & 6673 & 6937 \\
\hline $\begin{array}{l}\text { XXI: Factors influencing health status } \\
\text { and contact with health services (ZOO- } \\
\text { Z99) }\end{array}$ & 12851 & 1242 & 8296 & 5293 & 4974 & 2422 \\
\hline $\begin{array}{l}\text { Total (ekskl. kap. XX) } \\
(\text { AOO-Z99 ekcl. V, W, X og Y) }\end{array}$ & 94197 & 102339 & 97397 & 57074 & 64183 & 88072 \\
\hline
\end{tabular}

1 Only discharges with a length of stay less than 90 days

Source: The national in-patient registers 
Table 3.4.2.c Bed days in hospitals per 100000 inhabitants by main diagnosis, women

\begin{tabular}{|c|c|c|c|c|c|c|}
\hline ICD-10 code & Denmark & Finland & Åland & Iceland $^{1}$ & Norway & Sweden \\
\hline Main diagnosis & 2014 & 2014 & $2010-14$ & 2014 & 2014 & 2014 \\
\hline $\begin{array}{l}\text { I: Certain infectious and } \\
\text { parasitic diseases (A00-B99) }\end{array}$ & 3115 & 3608 & 3179 & 889 & 2103 & 2595 \\
\hline II: Neoplasms (C00-D48) & 6331 & 9751 & 11229 & 8651 & 8324 & 8470 \\
\hline $\begin{array}{l}\text { III: Diseases of the blood and } \\
\text { blood forming organs and certain } \\
\text { disorders involving the } \\
\text { immune mechanism (D50-D89) }\end{array}$ & 703 & 706 & 620 & 574 & 590 & 689 \\
\hline $\begin{array}{l}\text { IV: Endocrine, nutritional and } \\
\text { metabolic diseases (E00-E90) }\end{array}$ & 2544 & 1929 & 1966 & 1832 & 1366 & 1710 \\
\hline $\begin{array}{l}\text { V: Mental and behavioural disorders } \\
\text { (F00-F99) }\end{array}$ & 297 & 27193 & 9276 & 3279 & 640 & 14732 \\
\hline $\begin{array}{l}\text { VI: Diseases of the nervous system } \\
\text { (G00-G99) }\end{array}$ & 2638 & 7510 & 26538 & 2936 & 2250 & 2270 \\
\hline $\begin{array}{l}\text { VII: Diseases of the eye and adnexa } \\
\text { (H00-H59) }\end{array}$ & 154 & 411 & 147 & 240 & 337 & 208 \\
\hline $\begin{array}{l}\text { VIII: Diseases of the ear and mastoid } \\
\text { process }(\mathrm{H} 60-\mathrm{H} 95)\end{array}$ & 176 & 213 & 313 & 215 & 173 & 215 \\
\hline $\begin{array}{l}\text { IX: Diseases of the circulatory system } \\
(100-199)\end{array}$ & 7088 & 18341 & 19247 & 7929 & 7587 & 11189 \\
\hline $\begin{array}{l}\mathrm{X} \text { : Diseases of the respiratory system } \\
\text { (J00-J99) }\end{array}$ & 7308 & 6301 & 6116 & 5019 & 6607 & 5610 \\
\hline $\begin{array}{l}\mathrm{XI} \text { : Diseases of the digestive system } \\
(\text { KOO-K93) }\end{array}$ & 6429 & 5531 & 6744 & 4315 & 5329 & 5666 \\
\hline $\begin{array}{l}\text { XII: Diseases of the skin and } \\
\text { subcutaneous tissue (L00-L99) }\end{array}$ & 850 & 1041 & 644 & 971 & 941 & 851 \\
\hline $\begin{array}{l}\text { XIII: Diseases of the musculoskeletal } \\
\text { system and connective tissue (M00-M99) }\end{array}$ & 4607 & 6344 & 7580 & 4622 & 5118 & 5023 \\
\hline $\begin{array}{l}\text { XIV: Diseases of the genitourinary } \\
\text { system (N00-N99) }\end{array}$ & 3525 & 4437 & 6168 & 2683 & 3277 & 3288 \\
\hline $\begin{array}{l}X V \text { : Pregnancy, childbirth and } \\
\text { the puerperium (000-099) }\end{array}$ & 6330 & 10050 & 10971 & 6279 & 3988 & 6920 \\
\hline $\begin{array}{l}\mathrm{XVI} \text { : Certain conditions originating in } \\
\text { the perinatal period }(P 00-P 96)\end{array}$ & 1352 & 1223 & 956 & 1588 & 1458 & 1379 \\
\hline $\begin{array}{l}\text { XVII: Congenital malformations, } \\
\text { deformations and } \\
\text { chromosomal abnormalities (Q00-Q99) }\end{array}$ & 451 & 575 & 282 & 491 & 572 & 445 \\
\hline $\begin{array}{l}\text { XVIII: Symptoms, signs and abnormal } \\
\text { clinical and laboratory findings, not } \\
\text { elsewhere classified (R00-R99) }\end{array}$ & 5578 & 3743 & 5837 & 3319 & 2645 & 4250 \\
\hline $\begin{array}{l}\text { XIX: Injury, poisoning and certain } \\
\text { other consequences of external causes } \\
\text { (SOO-T98) }\end{array}$ & 6912 & 10418 & 7903 & 6584 & 7109 & 8875 \\
\hline $\begin{array}{l}\text { XXI: Factors influencing health status } \\
\text { and contact with health services (Z00- } \\
\text { Z99) }\end{array}$ & 11974 & 1428 & 12645 & 6707 & 9671 & 2428 \\
\hline $\begin{array}{l}\text { All causes (except. } \mathrm{XX} \text { ) } \\
\text { (A00-Z99 excluding } \mathrm{V}, \mathrm{W}, \mathrm{X} \text { and } \mathrm{Y})\end{array}$ & 97091 & 120752 & 138362 & 69125 & 70085 & 94776 \\
\hline
\end{tabular}

1 Only discharges with a length of stay less than 90 days

Source: The national in-patient registers 
Table 3.4.3.a Average length of stay per discharge (in days) per 100000 inhabitants by main diagnosis, both genders

\begin{tabular}{|c|c|c|c|c|c|c|}
\hline ICD-10 code & Denmark & Finland & Åland & Iceland $^{1}$ & Norway & Sweden \\
\hline Main diagnosis & 2014 & 2014 & $2010-14$ & 2014 & 2014 & 2014 \\
\hline $\begin{array}{l}\text { I: Certain infectious and } \\
\text { parasitic diseases (A00-B99) }\end{array}$ & 4.2 & 7.0 & 6.8 & 5.9 & 5.2 & 5.5 \\
\hline II: Neoplasms (C00-D48) & 4.0 & 6.1 & 9.4 & 8.1 & 5.4 & 6.9 \\
\hline $\begin{array}{l}\text { III: Diseases of the blood and } \\
\text { blood forming organs and certain } \\
\text { disorders involving the } \\
\text { immune mechanism (D50-D89) }\end{array}$ & 2.6 & 4.8 & 5.9 & 6.5 & 3.6 & 4.3 \\
\hline $\begin{array}{l}\text { IV: Endocrine, nutritional and } \\
\text { metabolic diseases (E00-E90) }\end{array}$ & 3.2 & 6.1 & 7.6 & 6.7 & 3.5 & 4.4 \\
\hline $\begin{array}{l}\text { V: Mental and behavioural disorders } \\
\text { (F00-F99) }\end{array}$ & 13.9 & 30.0 & 14.6 & 15.2 & 2.1 & 12.4 \\
\hline $\begin{array}{l}\text { VI: Diseases of the nervous system } \\
\text { (G00-G99) }\end{array}$ & 4.1 & 10.9 & 37.1 & 8.7 & 3.4 & 5.0 \\
\hline $\begin{array}{l}\text { VII: Diseases of the eye and adnexa } \\
(\mathrm{H} 00-\mathrm{H} 59)\end{array}$ & 1.8 & 2.8 & 3.3 & 3.6 & 3.0 & 2.4 \\
\hline $\begin{array}{l}\text { VIII: Diseases of the ear and mastoid } \\
\text { process (H60-H95) }\end{array}$ & 1.7 & 3.1 & 2.5 & 4.6 & 1.9 & 2.2 \\
\hline $\begin{array}{l}\text { IX: Diseases of the circulatory system } \\
(100-199)\end{array}$ & 3.5 & 7.8 & 8.4 & 8.8 & 4.1 & 5.5 \\
\hline $\begin{array}{l}X: \text { Diseases of the respiratory system } \\
(\mathrm{J} 00-\mathrm{J} 99)\end{array}$ & 3.7 & 6.3 & 6.2 & 7.8 & 5.4 & 5.3 \\
\hline $\begin{array}{l}\mathrm{XI} \text { : Diseases of the digestive system } \\
\text { (K00-K93) }\end{array}$ & 3.2 & 4.8 & 5.1 & 5.2 & 4.0 & 4.3 \\
\hline $\begin{array}{l}\text { XII: Diseases of the skin and } \\
\text { subcutaneous tissue (LOO-L99) }\end{array}$ & 3.0 & 7.1 & 7.7 & 6.4 & 5.7 & 6.0 \\
\hline $\begin{array}{l}\text { XIII: Diseases of the musculoskeletal } \\
\text { system and connective tissue (M00-M99) }\end{array}$ & 3.1 & 5.0 & 4.6 & 6.3 & 3.9 & 4.4 \\
\hline $\begin{array}{l}\text { XIV: Diseases of the genitourinary } \\
\text { system (N00-N99) }\end{array}$ & 2.6 & 4.7 & 4.7 & 4.0 & 3.4 & 4.2 \\
\hline $\begin{array}{l}X V \text { : Pregnancy, childbirth and } \\
\text { the puerperium (000-099) }\end{array}$ & 2.4 & 4.0 & 4.7 & 2.0 & 3.2 & 2.4 \\
\hline $\begin{array}{l}\mathrm{XVI} \text { : Certain conditions originating in } \\
\text { the perinatal period }(P 00-P 96)\end{array}$ & 8.1 & 8.3 & 9.1 & 4.8 & 9.4 & 9.6 \\
\hline $\begin{array}{l}\text { XVII: Congenital malformations, } \\
\text { deformations and } \\
\text { chromosomal abnormalities (Q00-Q99) }\end{array}$ & 2.9 & 4.7 & 6.2 & 4.0 & 4.5 & 5.0 \\
\hline $\begin{array}{l}\text { XVIII: Symptoms, signs and abnormal } \\
\text { clinical and laboratory findings, not } \\
\text { elsewhere classified (R00-R99) }\end{array}$ & 1.8 & 3.7 & 3.8 & 4.5 & 1.7 & 2.6 \\
\hline $\begin{array}{l}\text { XIX: Injury, poisoning and certain } \\
\text { other consequences of external causes } \\
\text { (SOO-T98) }\end{array}$ & 3.1 & 6.7 & 6.9 & 7.4 & 3.9 & 5.0 \\
\hline $\begin{array}{l}\text { XXI: Factors influencing health status } \\
\text { and contact with health services (Z0O- } \\
\text { Z99) }\end{array}$ & 4.4 & 6.1 & 12.4 & 7.4 & 4.4 & 3.9 \\
\hline $\begin{array}{l}\text { All causes (except. } \mathrm{XX} \text { ) } \\
\text { (A00-Z99 excluding } \mathrm{V}, \mathrm{W}, \mathrm{X} \text { and } \mathrm{Y} \text { ) }\end{array}$ & 3.7 & 7.3 & 7.8 & 6.2 & 4.0 & 5.4 \\
\hline
\end{tabular}

1 Only discharges with a length of stay less than 90 days

Source: The national in-patient registers 
Table 3.4.3.b Average length of stay per discharge (in days) per 100000 inhabitants by main diagnosis, men

\begin{tabular}{|c|c|c|c|c|c|c|}
\hline ICD-10 code & Denmark & Finland & Åland & Iceland $^{1}$ & Norway & Sweden \\
\hline Main diagnosis & 2014 & 2014 & 2010-14 & 2014 & 2014 & 2014 \\
\hline $\begin{array}{l}\text { I: Certain infectious and } \\
\text { parasitic diseases (A00-B99) }\end{array}$ & 4.3 & 7.1 & 7.3 & 6.4 & 5.4 & 5.4 \\
\hline II: Neoplasms (C00-D48) & 4.3 & 6.2 & 10.3 & 8.1 & 5.7 & 7.2 \\
\hline $\begin{array}{l}\text { III: Diseases of the blood and } \\
\text { blood forming organs and certain } \\
\text { disorders involving the } \\
\text { immune mechanism (D50-D89) }\end{array}$ & 2.6 & 4.6 & 7.0 & 6.3 & 3.9 & 4.5 \\
\hline $\begin{array}{l}\text { IV: Endocrine, nutritional and } \\
\text { metabolic diseases (E00-E90) }\end{array}$ & 3.4 & 6.6 & 7.0 & 7.9 & 3.8 & 4.9 \\
\hline $\begin{array}{l}\text { V: Mental and behavioural disorders } \\
\text { (F00-F99) }\end{array}$ & 12.7 & 26.9 & 12.2 & 15.9 & 1.8 & 11.8 \\
\hline $\begin{array}{l}\text { VI: Diseases of the nervous system } \\
\text { (G00-G99) }\end{array}$ & 4.1 & 9.1 & 23.3 & 8.7 & 3.3 & 5.2 \\
\hline $\begin{array}{l}\text { VII: Diseases of the eye and adnexa } \\
\text { (H00-H59) }\end{array}$ & 1.9 & 2.7 & 3.4 & 2.9 & 2.9 & 2.2 \\
\hline $\begin{array}{l}\text { VIII: Diseases of the ear and mastoid } \\
\text { process }(\mathrm{H} 60-\mathrm{H} 95)\end{array}$ & 1.8 & 3.3 & 2.4 & 3.3 & 1.9 & 2.2 \\
\hline $\begin{array}{l}\text { IX: Diseases of the circulatory system } \\
(100-199)\end{array}$ & 3.5 & 6.9 & 6.9 & 8.5 & 4.0 & 5.2 \\
\hline $\begin{array}{l}X: \text { Diseases of the respiratory system } \\
(\mathrm{J} 00-\mathrm{J} 99)\end{array}$ & 3.7 & 6.1 & 6.3 & 7.3 & 5.4 & 5.2 \\
\hline $\begin{array}{l}\mathrm{XI} \text { : Diseases of the digestive system } \\
(\text { K00-K93) }\end{array}$ & 3.2 & 4.8 & 5.3 & 5.2 & 3.9 & 4.2 \\
\hline $\begin{array}{l}\text { XII: Diseases of the skin and } \\
\text { subcutaneous tissue (LOO-L99) }\end{array}$ & 3.1 & 6.7 & 8.2 & 6.0 & 5.6 & 5.9 \\
\hline $\begin{array}{l}\text { XIII: Diseases of the musculoskeletal } \\
\text { system and connective tissue (M00-M99) }\end{array}$ & 2.9 & 4.8 & 4.1 & 5.7 & 3.9 & 4.2 \\
\hline $\begin{array}{l}\text { XIV: Diseases of the genitourinary } \\
\text { system (N00-N99) }\end{array}$ & 2.8 & 4.8 & 5.6 & 4.5 & 3.7 & 4.4 \\
\hline $\begin{array}{l}X V \text { : Pregnancy, childbirth and } \\
\text { the puerperium (000-099) }\end{array}$ & .. & .. & .. & .. & .. &.. \\
\hline $\begin{array}{l}\mathrm{XVI} \text { : Certain conditions originating in } \\
\text { the perinatal period }(P 00-P 96)\end{array}$ & 7.7 & 8.4 & 9.5 & 4.6 & 9.5 & 9.7 \\
\hline $\begin{array}{l}\text { XVII: Congenital malformations, } \\
\text { deformations and } \\
\text { chromosomal abnormalities (Q00-Q99) }\end{array}$ & 2.8 & 4.8 & 6.7 & 4.2 & 4.3 & 5.1 \\
\hline $\begin{array}{l}\text { XVIII: Symptoms, signs and abnormal } \\
\text { clinical and laboratory findings, not } \\
\text { elsewhere classified (R00-R99) }\end{array}$ & 1.9 & 3.6 & 3.8 & 4.5 & 1.7 & 2.5 \\
\hline $\begin{array}{l}\text { XIX: Injury, poisoning and certain } \\
\text { other consequences of external causes } \\
\text { (SOO-T98) }\end{array}$ & 3.0 & 6.3 & 7.6 & 7.2 & 3.8 & 4.6 \\
\hline $\begin{array}{l}\text { XXI: Factors influencing health status } \\
\text { and contact with health services (Z0O- } \\
\text { Z99) }\end{array}$ & 4.7 & 6.7 & 12.2 & 8.4 & 6.1 & 4.1 \\
\hline $\begin{array}{l}\text { All causes (except. } \mathrm{XX} \text { ) } \\
\text { (A00-Z99 excluding } \mathrm{V}, \mathrm{W}, \mathrm{X} \text { and } \mathrm{Y} \text { ) }\end{array}$ & 3.8 & 7.3 & 7.5 & 7.0 & 4.2 & 5.6 \\
\hline
\end{tabular}

1 Only discharges with a length of stay less than 90 days

Source: The national in-patient registers 
Table 3.4.3.c Average length of stay per discharge (in days) per 100000 inhabitants by main diagnosis, women

\begin{tabular}{|c|c|c|c|c|c|c|}
\hline ICD-10 code & Denmark & Finland & Åland & Iceland $^{1}$ & Norway & Sweden \\
\hline Main diagnosis & 2014 & 2014 & $2010-14$ & 2014 & 2014 & 2014 \\
\hline $\begin{array}{l}\text { I: Certain infectious and } \\
\text { parasitic diseases (A00-B99) }\end{array}$ & 4.1 & 6.9 & 6.2 & 5.4 & 5.1 & 5.5 \\
\hline II: Neoplasms (C00-D48) & 3.6 & 6.0 & 8.8 & 8.2 & 5.1 & 6.6 \\
\hline $\begin{array}{l}\text { III: Diseases of the blood and } \\
\text { blood forming organs and certain } \\
\text { disorders involving the } \\
\text { immune mechanism (D50-D89) }\end{array}$ & 2.6 & 4.9 & 4.8 & 6.7 & 3.3 & 4.2 \\
\hline $\begin{array}{l}\text { IV: Endocrine, nutritional and } \\
\text { metabolic diseases (E00-E90) }\end{array}$ & 3.0 & 5.7 & 8.0 & 6.2 & 3.3 & 4.1 \\
\hline $\begin{array}{l}\text { V: Mental and behavioural disorders } \\
\text { (F00-F99) }\end{array}$ & 15.1 & 33.2 & 16.8 & 14.6 & 2.5 & 13.1 \\
\hline $\begin{array}{l}\text { VI: Diseases of the nervous system } \\
\text { (G00-G99) }\end{array}$ & 4.2 & 12.6 & 48.2 & 8.6 & 3.5 & 4.8 \\
\hline $\begin{array}{l}\text { VII: Diseases of the eye and adnexa } \\
(\mathrm{H} 00-\mathrm{H} 59)\end{array}$ & 1.8 & 2.8 & 3.2 & 4.2 & 3.0 & 2.5 \\
\hline $\begin{array}{l}\text { VIII: Diseases of the ear and mastoid } \\
\text { process (H60-H95) }\end{array}$ & 1.6 & 3.0 & 2.6 & 5.5 & 2.0 & 2.3 \\
\hline $\begin{array}{l}\text { IX: Diseases of the circulatory system } \\
(100-199)\end{array}$ & 3.5 & 9.0 & 10.0 & 9.3 & 4.2 & 5.8 \\
\hline $\begin{array}{l}\mathrm{X} \text { : Diseases of the respiratory system } \\
\text { (J00-J99) }\end{array}$ & 3.6 & 6.5 & 6.1 & 8.4 & 5.4 & 5.4 \\
\hline $\begin{array}{l}\mathrm{XI}: \text { Diseases of the digestive system } \\
(\text { KOO-K93) }\end{array}$ & 3.3 & 4.7 & 4.9 & 5.3 & 4.0 & 4.4 \\
\hline $\begin{array}{l}\text { XII: Diseases of the skin and } \\
\text { subcutaneous tissue (LOO-L99) }\end{array}$ & 2.9 & 7.6 & 7.2 & 6.8 & 5.9 & 6.1 \\
\hline $\begin{array}{l}\text { XIII: Diseases of the musculoskeletal } \\
\text { system and connective tissue (M00-M99) }\end{array}$ & 3.2 & 5.1 & 5.0 & 6.8 & 3.9 & 4.6 \\
\hline $\begin{array}{l}\text { XIV: Diseases of the genitourinary } \\
\text { system (NOO-N99) }\end{array}$ & 2.4 & 4.6 & 4.3 & 3.8 & 3.2 & 4.0 \\
\hline $\begin{array}{l}\mathrm{XV} \text { : Pregnancy, childbirth and } \\
\text { the puerperium (000-099) }\end{array}$ & 2.4 & 4.0 & 4.7 & 2.0 & 3.2 & 2.4 \\
\hline $\begin{array}{l}\mathrm{XVI} \text { : Certain conditions originating in } \\
\text { the perinatal period }(P 00-P 96)\end{array}$ & 8.8 & 8.3 & 8.7 & 5.1 & 9.3 & 9.6 \\
\hline $\begin{array}{l}\text { XVII: Congenital malformations, } \\
\text { deformations and } \\
\text { chromosomal abnormalities (Q00-Q99) }\end{array}$ & 3.0 & 4.5 & 5.7 & 3.7 & 4.6 & 4.9 \\
\hline $\begin{array}{l}\text { XVIII: Symptoms, signs and abnormal } \\
\text { clinical and laboratory findings, not } \\
\text { elsewhere classified (R00-R99) }\end{array}$ & 1.8 & 3.8 & 3.7 & 4.4 & 1.7 & 2.6 \\
\hline $\begin{array}{l}\text { XIX: Injury, poisoning and certain } \\
\text { other consequences of external causes } \\
\text { (SOO-T98) }\end{array}$ & 3.2 & 7.1 & 6.1 & 7.5 & 4.0 & 5.4 \\
\hline $\begin{array}{l}\text { XXI: Factors influencing health status } \\
\text { and contact with health services (Z0O- } \\
\text { Z99) }\end{array}$ & 4.0 & 5.6 & 12.5 & 6.8 & 3.9 & 3.8 \\
\hline $\begin{array}{l}\text { All causes (except. } \mathrm{XX} \text { ) } \\
\text { (A00-Z99 excluding } \mathrm{V}, \mathrm{W}, \mathrm{X} \text { and } \mathrm{Y} \text { ) }\end{array}$ & 3.6 & 7.4 & 8.1 & 5.7 & 3.9 & 5.1 \\
\hline
\end{tabular}

1 Only discharges with a length of stay less than 90 days

Source: The national in-patient registers 
Morbidity, Medical Treatment, Accidents and Pharmaceutical products

Figure 3.4.1 Number of bed days for cancer per 1000 inhabitants, 2003-20141

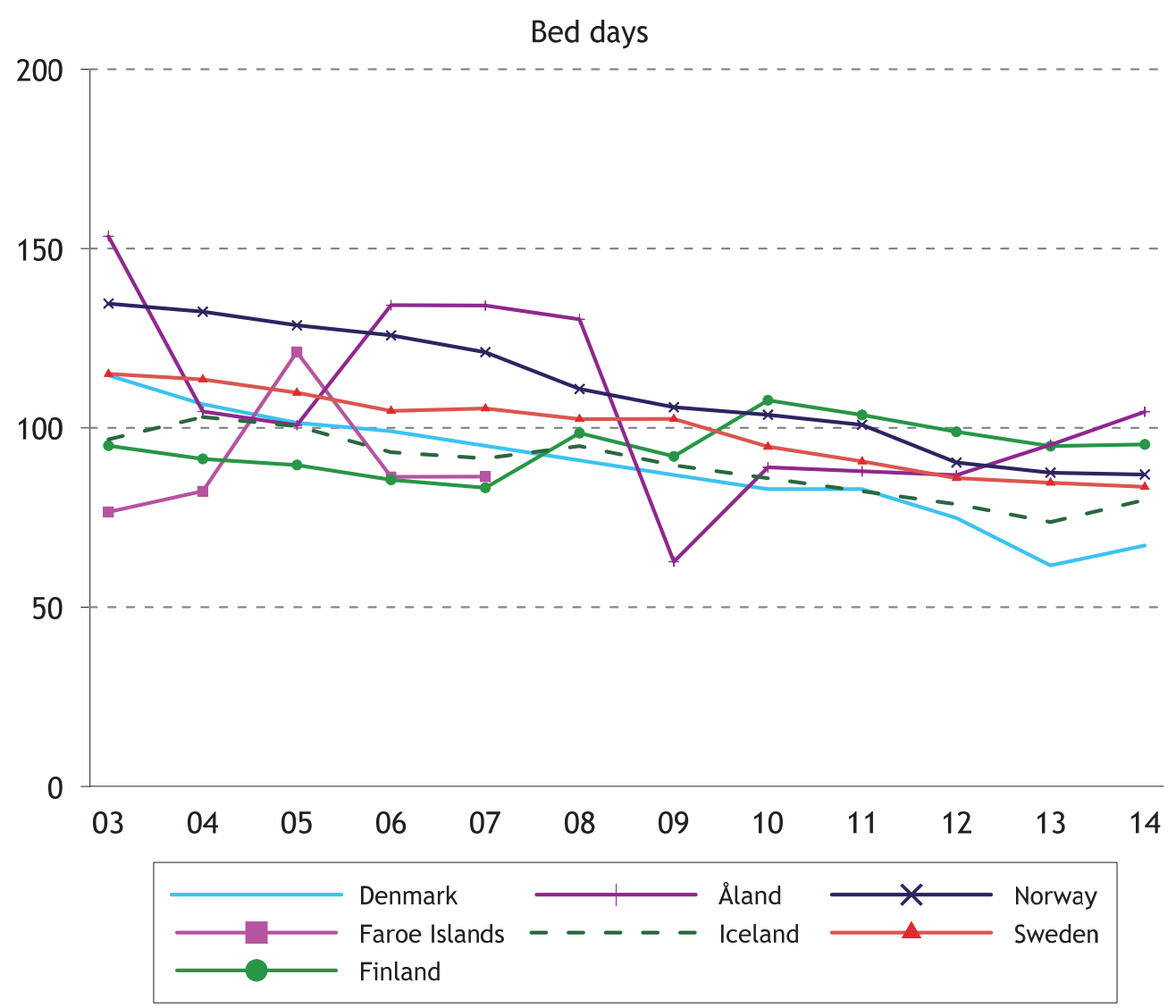

1 Iceland: Only discharges with a length of stay less than 90 days

ICD10 C00-D48

Source: The national in-patient registers 
Figure 3.4.2 Bed days during the year for diseases of the circulation organs per 1000 inhabitants, 2003-2014 1

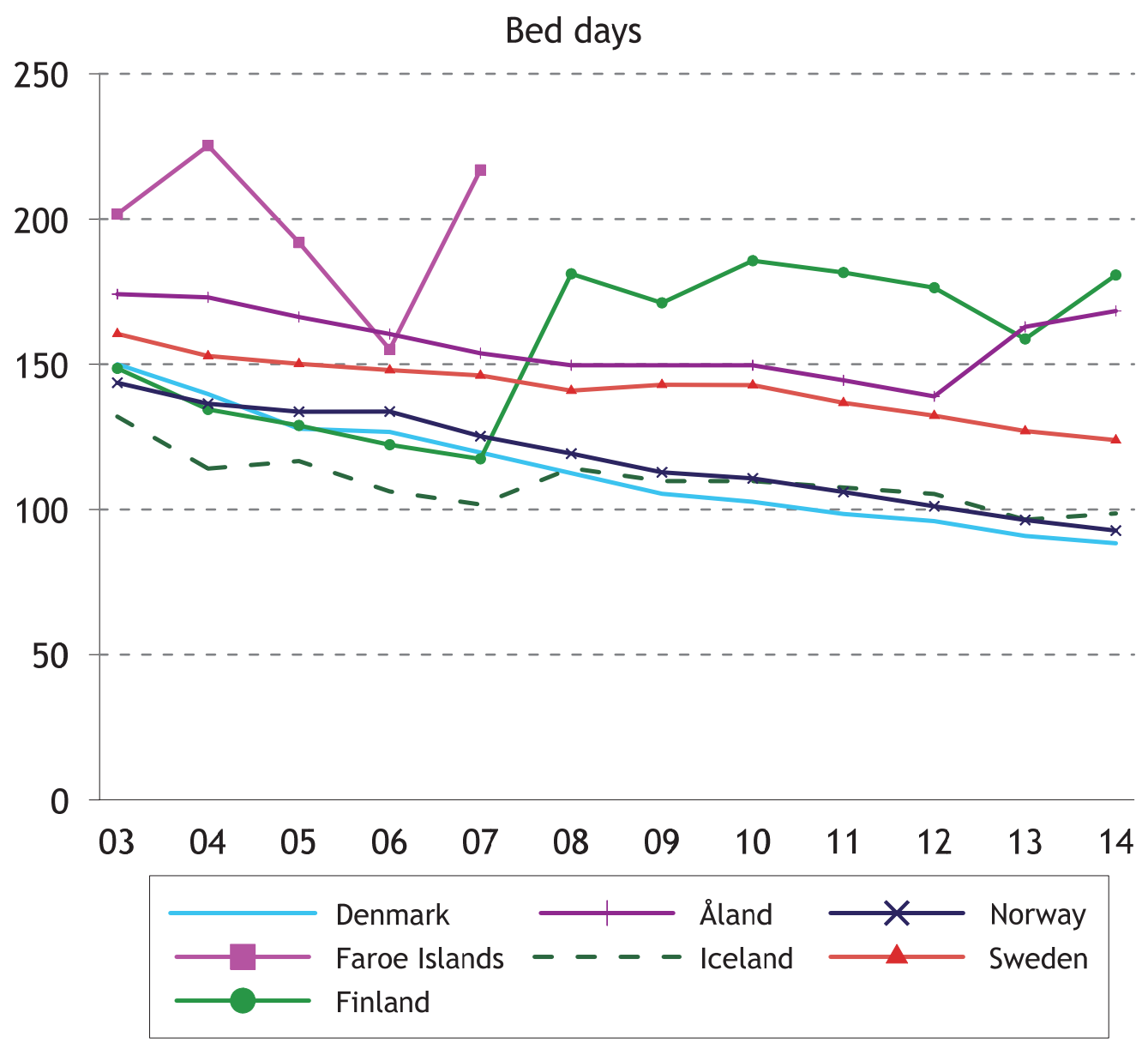

1 Iceland: Only discharges with a length of stay less than 90 days ICD10 100-199

Source: The national in-patient registers 
Morbidity, Medical Treatment, Accidents and Pharmaceutical products

Figure 3.4.3 Bed days for diseases of the digestive system per 1000 inhabitants 2003-2014

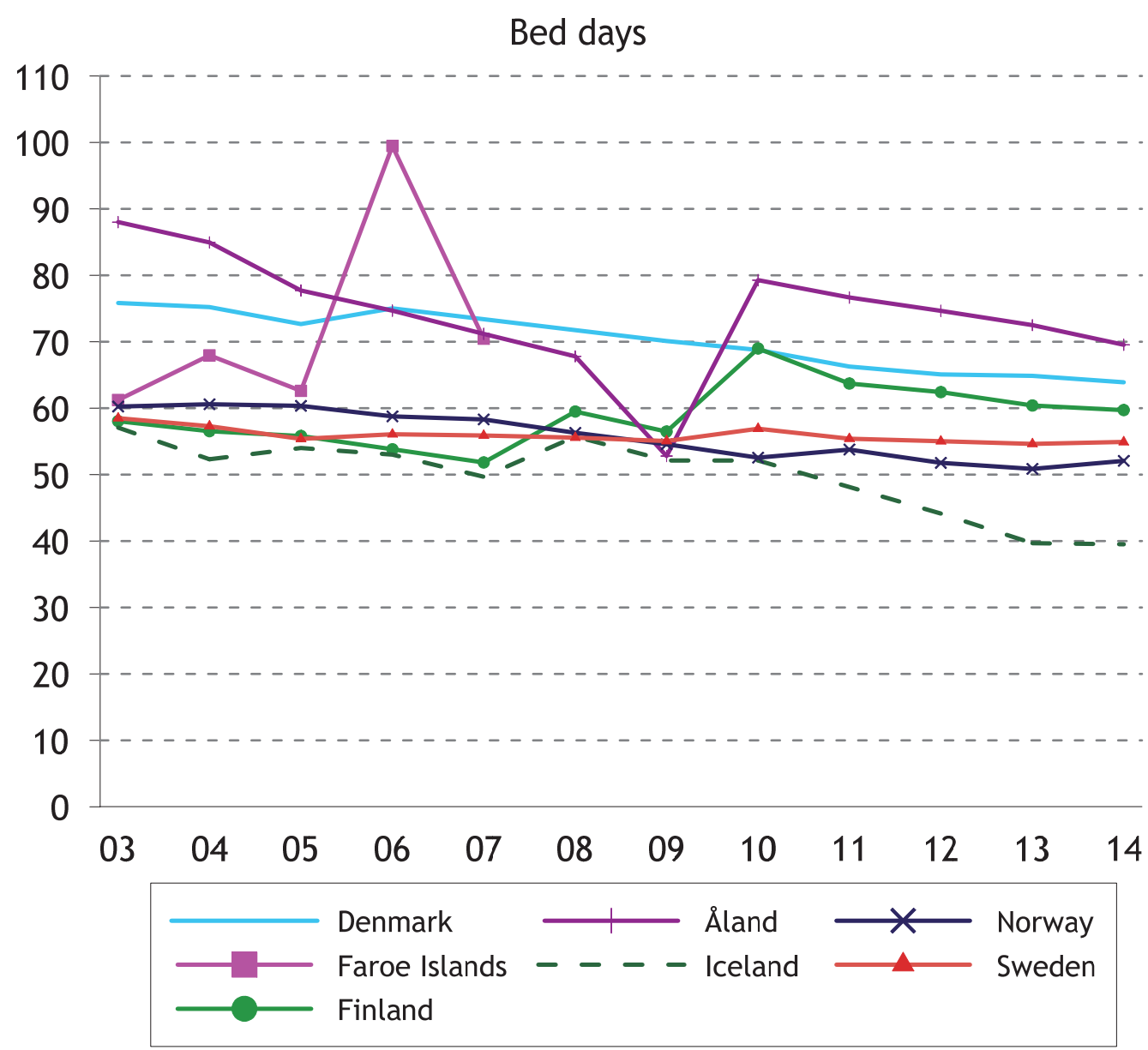

1 Iceland: Only discharges with a length of stay less than 90 days ICD10 K00-K93

Source: The national in-patient registers 
Figure 3.4.4 Discharges for cancer per 1000 inhabitants $2000-2014^{1}$

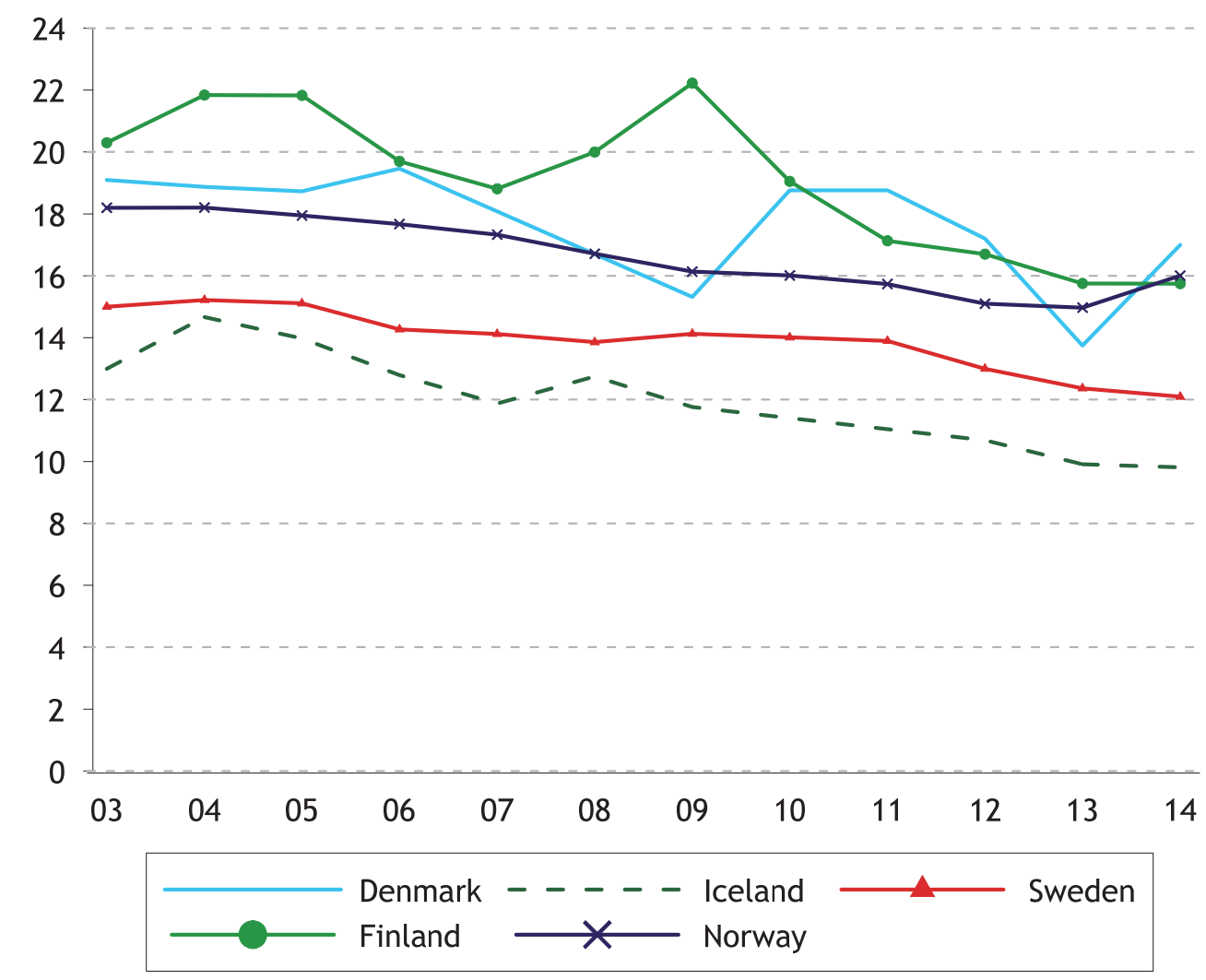

1 Iceland: Only discharges with a length of stay less than 90 days

ICD10 C00-D48

Source: The national in-patient registers 
Morbidity, Medical Treatment, Accidents and Pharmaceutical products

Figure 3.4.5 Discharges during the year for diseases of the circulation organs per 1000 inhabitants, 2003-2014 1

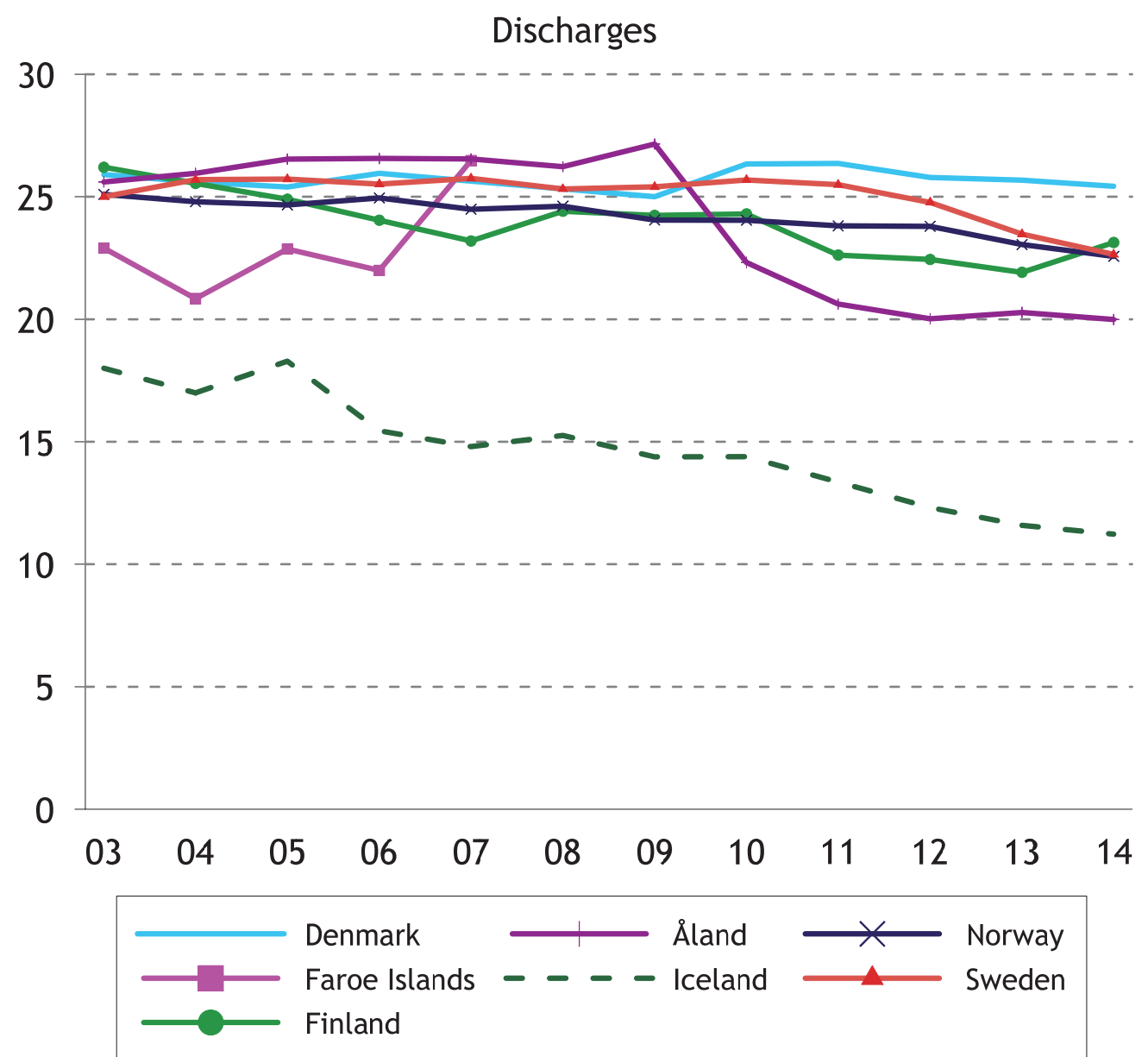

1 Iceland: Only discharges with a length of stay less than 90 days

ICD10 100-199

Source: The national in-patient registers 
Figure 3.4.6 Discharges for diseases of the digestive system per 1000 inhabitants 2003-2014

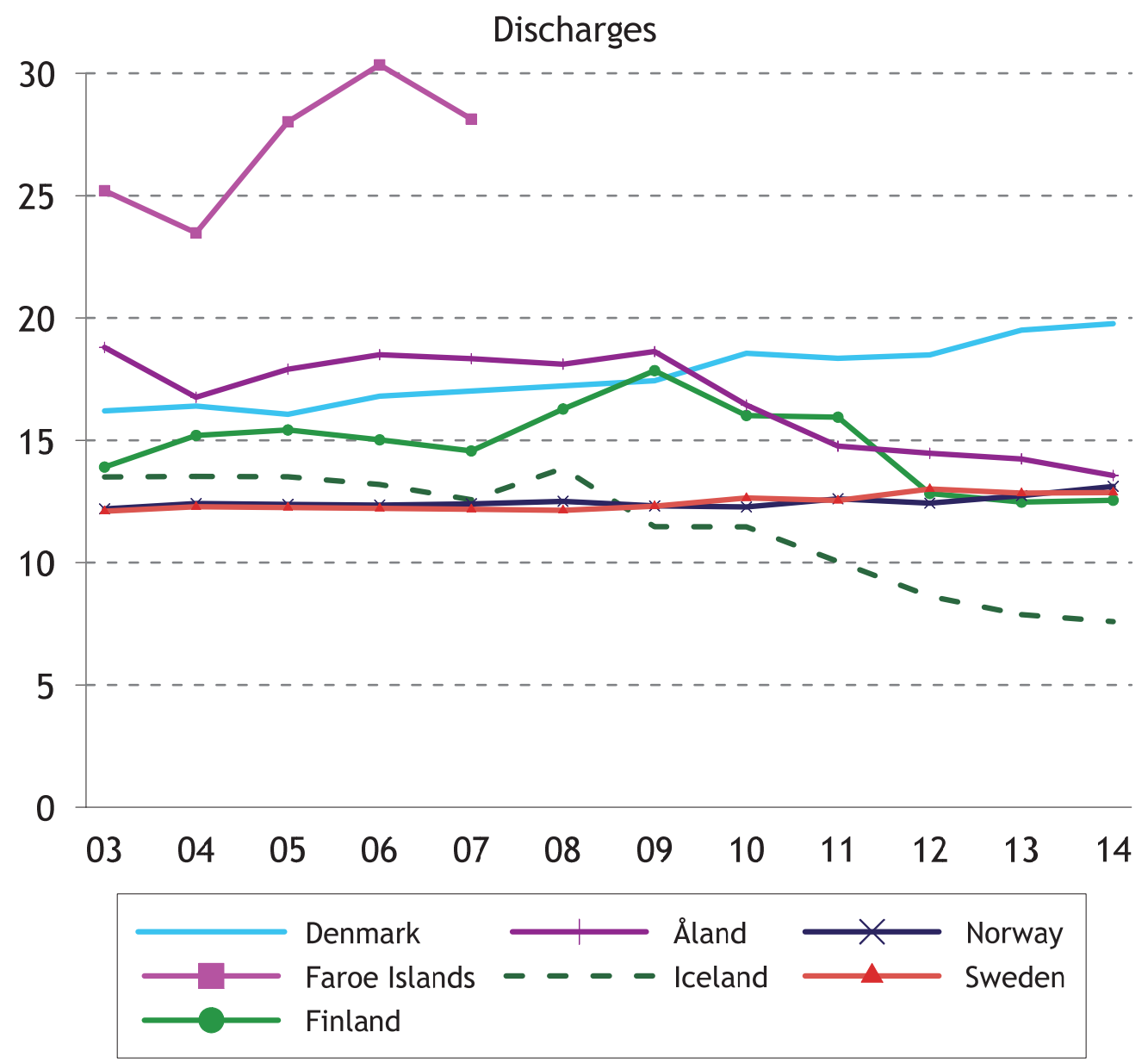

1 Iceland: Only discharges with a length of stay less than 90 days

ICD10 K00-K93

Source: The national in-patient registers 
Table 3.4.4 Discharges, bed days and average length of stay in wards in ordinary hospitals and special hospitals, 2014

\begin{tabular}{|c|c|c|c|c|c|c|c|c|}
\hline & Denmark & $\begin{array}{l}\text { Faroe } \\
\text { Islands }\end{array}$ & $\begin{array}{l}\text { Green- } \\
\text { land }^{1,2}\end{array}$ & Finland & Åland ${ }^{3}$ & Iceland & Norway $^{4}$ & Sweden \\
\hline \multicolumn{9}{|l|}{$\begin{array}{l}\text { Discharges per } \\
1000 \text { inhabitants }\end{array}$} \\
\hline Somatic wards & 257 & 233 & 304 & 174 & 172 & 113 & 166 & 161 \\
\hline Psychiatry wards & 9 & 5 & 3 & 7 & 10 & 7 & 13 & 11 \\
\hline Total & 267 & 238 & 307 & 181 & 182 & 120 & 179 & 171 \\
\hline \multicolumn{9}{|l|}{$\begin{array}{l}\text { Bed days per } \\
1000 \text { inhabitants }\end{array}$} \\
\hline Somatic wards & 957 & 994 & 1532 & 701 & 1048 & 655 & 671 & 770 \\
\hline Psychiatry wards & 140 & 239 & 71 & 238 & 187 & 80 & 271 & 148 \\
\hline Total & 1097 & 1233 & 1603 & 939 & 1234 & 735 & 942 & 919 \\
\hline \multicolumn{9}{|c|}{ Average length of stay } \\
\hline Somatic wards & 4 & 4 & 5 & 4 & 6 & 6 & 4 & 5 \\
\hline Psychiatry wards & 16 & 44 & 24 & 35 & 20 & 11 & 21 & 14 \\
\hline Total & 4 & 5 & 5 & 5 & 7 & 6 & 5 & 5 \\
\hline
\end{tabular}

1 The average length of stay was not recorded in psychiatry wards in 2014, the figure is the average sum of days in 2010-2013

2 Somatic wards are throughout Greenland. Psychiatric ward is only at DIH (Dronning Ingrids Hospital)

3 Average 2010-14

4 Figures for psychiatry include activity in psychiatric hospitals, psychiatric wards and community mental health care centre. Beds for adults, children and people receiving treatment for addiction are included. Figures for somatic wards include activity in somatic hospitals (not including rehabilitation). A patient is only counted once in somatic and/or psychiatric wards

Source: The national in-patient registers 
Table 3.4.5 Discharges, patients treated and average length of stay in hospital for malignant neoplasm of trachea, bronchus and lungs, 2014

\begin{tabular}{|c|c|c|c|c|c|c|}
\hline & Denmark & Finland & Åland ${ }^{1}$ & Iceland $^{2}$ & Norway & Sweden \\
\hline $\begin{array}{l}\text { Discharges } \\
\text { Men, total } \\
\text { Women, total }\end{array}$ & $\begin{array}{l}2827 \\
2793\end{array}$ & $\begin{array}{l}2899 \\
1637\end{array}$ & $\begin{array}{r}11 \\
9\end{array}$ & $\begin{array}{l}139 \\
190\end{array}$ & $\begin{array}{l}3547 \\
3097\end{array}$ & $\begin{array}{l}3855 \\
4309\end{array}$ \\
\hline $\begin{array}{l}\text { Patients treated } \\
\text { Men, total } \\
\text { Women, total }\end{array}$ & $\begin{array}{l}1762 \\
1821\end{array}$ & $\begin{array}{r}1574 \\
919\end{array}$ & $\begin{array}{l}7 \\
5\end{array}$ & $\begin{array}{r}85 \\
111\end{array}$ & $\begin{array}{l}1855 \\
1604\end{array}$ & $\begin{array}{l}2308 \\
2516\end{array}$ \\
\hline $\begin{array}{l}\text { Patients treated pe } \\
100 \text { o00 men in the } \\
\text { age group } \\
25-44 \\
45-64 \\
65+ \\
\text { Total rate }\end{array}$ & $\begin{array}{r}3 \\
63 \\
272 \\
63\end{array}$ & $\begin{array}{r}2 \\
58 \\
248 \\
59\end{array}$ & $\begin{array}{r}0 \\
56 \\
174 \\
47\end{array}$ & $\begin{array}{r}0 \\
66 \\
310 \\
53\end{array}$ & $\begin{array}{r}2 \\
69 \\
371 \\
72\end{array}$ & $\begin{array}{r}2 \\
38 \\
202 \\
48\end{array}$ \\
\hline $\begin{array}{l}\text { Patients treated pe } \\
100 \text { 000 women } \\
\text { in the age group } \\
25-44 \\
45-64 \\
65+ \\
\text { Total rate }\end{array}$ & $\begin{array}{r}4 \\
75 \\
221 \\
64\end{array}$ & $\begin{array}{r}1 \\
33 \\
110 \\
33\end{array}$ & $\begin{array}{r}0 \\
48 \\
110 \\
35\end{array}$ & $\begin{array}{r}5 \\
89 \\
332 \\
69\end{array}$ & $\begin{array}{r}2 \\
74 \\
248 \\
63\end{array}$ & $\begin{array}{r}2 \\
50 \\
178 \\
52\end{array}$ \\
\hline $\begin{array}{l}\text { Average length of } \\
\text { stay per discharge }\end{array}$ & 5.5 & 8.4 & 11.2 & 9.5 & 6.6 & 9.8 \\
\hline
\end{tabular}

1 Average 2010-14

2 Only discharges with a length of stay less than 90 days

ICD10 C33-C34

Source: The national in-patient registers

Table 3.4.6 Discharges, patients treated and average length of stay in hospital for malignant neoplasm of breast, women 2014

\begin{tabular}{lrrrrrr}
\hline & Denmark & Finland & Áland $^{1}$ & Iceland $^{2}$ & Norway & Sweden \\
\hline $\begin{array}{l}\text { Discharges } \\
\text { Total }\end{array}$ & 6565 & 8562 & 30 & 339 & 5114 & 7824 \\
$\begin{array}{l}\text { Patients treated } \\
\text { Total }\end{array}$ & 5043 & 6664 & 25 & 257 & 3685 & 6924 \\
$\begin{array}{l}\text { Patients treated per } \\
\text { 100 000 women }\end{array}$ & & & & & & \\
$\begin{array}{l}\text { in the age group } \\
\text { 25-44 }\end{array}$ & 63 & 70 & 58 & 69 & 60 & 45 \\
$\begin{array}{l}45-64 \\
65\end{array}$ & 412 & 439 & 270 & 270 & 258 & 209 \\
Total rate & 177 & 240 & 418 & 208 & 176 & 364 \\
$\begin{array}{l}\text { Average length of } \\
\text { stay per discharge }\end{array}$ & & & 177 & 161 & 144 & 143 \\
\hline
\end{tabular}

1 Average 2010-14

2 Only discharges with a length of stay less than 90 days

ICD10 C50

Source: The national in-patient registers 
Morbidity, Medical Treatment, Accidents and Pharmaceutical products

Table 3.4.7 Discharges, patients treated and average length of stay in hospital for acute myocardial infarction 2014

\begin{tabular}{|c|c|c|c|c|c|c|}
\hline & Denmark & Finland & Åland ${ }^{1}$ & Iceland ${ }^{2}$ & Norway & Sweden \\
\hline $\begin{array}{l}\text { Discharges } \\
\text { Men, Total } \\
\text { Women, Total }\end{array}$ & $\begin{array}{r}10847 \\
5335\end{array}$ & $\begin{array}{l}8388 \\
5046\end{array}$ & $\begin{array}{l}39 \\
31\end{array}$ & $\begin{array}{l}355 \\
153\end{array}$ & $\begin{array}{r}12832 \\
6036\end{array}$ & $\begin{array}{l}18991 \\
10779\end{array}$ \\
\hline $\begin{array}{l}\text { Patients treated } \\
\text { Men, Total } \\
\text { Women, Total }\end{array}$ & $\begin{array}{l}6036 \\
3180\end{array}$ & $\begin{array}{l}6234 \\
3871\end{array}$ & $\begin{array}{l}30 \\
23\end{array}$ & $\begin{array}{l}321 \\
135\end{array}$ & $\begin{array}{l}7460 \\
3910\end{array}$ & $\begin{array}{r}12797 \\
7488\end{array}$ \\
\hline $\begin{array}{l}\text { Patients treated pe } \\
100000 \text { men } \\
\text { in the age group } \\
0-44 \\
45-64 \\
65+ \\
\text { Total rate }\end{array}$ & $\begin{array}{r}19 \\
314 \\
718 \\
215\end{array}$ & $\begin{array}{r}13 \\
273 \\
877 \\
232\end{array}$ & $\begin{array}{r}10 \\
218 \\
815 \\
212\end{array}$ & $\begin{array}{r}11 \\
336 \\
931 \\
199\end{array}$ & $\begin{array}{r}20 \\
409 \\
1190 \\
289\end{array}$ & $\begin{array}{r}10 \\
298 \\
991 \\
264\end{array}$ \\
\hline $\begin{array}{l}\text { Patients treated pe } \\
100000 \text { women } \\
\text { in the age group }\end{array}$ & & & & & & \\
\hline $\begin{array}{l}0-44 \\
45-64 \\
65+ \\
\text { Total rate }\end{array}$ & $\begin{array}{r}7 \\
102 \\
410 \\
112\end{array}$ & $\begin{array}{r}2 \\
69 \\
545 \\
139\end{array}$ & $\begin{array}{r}0 \\
92 \\
658 \\
161\end{array}$ & $\begin{array}{r}3 \\
59 \\
496 \\
84\end{array}$ & $\begin{array}{r}4 \\
115 \\
689 \\
153\end{array}$ & $\begin{array}{r}2 \\
90 \\
599 \\
154\end{array}$ \\
\hline $\begin{array}{l}\text { Average length of } \\
\text { stay per discharge }\end{array}$ & 2.9 & 5.9 & 5.5 & 5.5 & 3.4 & 4.2 \\
\hline
\end{tabular}

1 Average 2010-14

2 Only discharges with a length of stay less than 90 days ICD10 121-122

Source: The national in-patient registers 
Table 3.4.8 Discharges, patients treated and average length of stay in hospital for cerebrovascular diseases 2014

\begin{tabular}{|c|c|c|c|c|c|c|}
\hline & Denmark & Finland & Åland ${ }^{1}$ & Iceland $^{2}$ & Norway & Sweden \\
\hline $\begin{array}{l}\text { Discharges } \\
\text { Men, Total } \\
\text { Women, Total }\end{array}$ & $\begin{array}{l}9811 \\
8674\end{array}$ & $\begin{array}{l}10919 \\
10209\end{array}$ & $\begin{array}{l}42 \\
43\end{array}$ & $\begin{array}{l}311 \\
225\end{array}$ & $\begin{array}{l}7747 \\
5528\end{array}$ & $\begin{array}{l}20442 \\
17872\end{array}$ \\
\hline $\begin{array}{l}\text { Patients treated } \\
\text { Men, Total } \\
\text { Women, Total }\end{array}$ & $\begin{array}{l}7362 \\
6497\end{array}$ & $\begin{array}{l}7664 \\
7247\end{array}$ & $\begin{array}{l}32 \\
33\end{array}$ & $\begin{array}{l}247 \\
172\end{array}$ & $\begin{array}{l}6296 \\
5528\end{array}$ & $\begin{array}{l}14734 \\
13293\end{array}$ \\
\hline $\begin{array}{l}\text { Patients treated } p \\
100 \text { o00 men } \\
\text { in the age group } \\
0-44 \\
45-64 \\
65-79 \\
80+ \\
\text { Total rate }\end{array}$ & $\begin{array}{r}23 \\
286 \\
854 \\
1818 \\
263\end{array}$ & $\begin{array}{r}23 \\
277 \\
958 \\
1943 \\
285\end{array}$ & $\begin{array}{r}21 \\
193 \\
745 \\
1627 \\
229\end{array}$ & $\begin{array}{r}18 \\
162 \\
605 \\
1660 \\
153\end{array}$ & $\begin{array}{r}19 \\
237 \\
875 \\
2310 \\
244\end{array}$ & $\begin{array}{r}14 \\
232 \\
923 \\
2525 \\
304\end{array}$ \\
\hline $\begin{array}{l}\text { Patients treated } p \\
100000 \text { women } \\
\text { in the age group } \\
0-44 \\
45-64 \\
65-79 \\
80+ \\
\text { Total rate }\end{array}$ & $\begin{array}{r}23 \\
186 \\
572 \\
1900 \\
229\end{array}$ & $\begin{array}{r}23 \\
176 \\
604 \\
1960 \\
261\end{array}$ & $\begin{array}{r}3 \\
149 \\
572 \\
1712 \\
229\end{array}$ & $\begin{array}{r}17 \\
61 \\
335 \\
1362 \\
107\end{array}$ & $\begin{array}{r}18 \\
141 \\
565 \\
2216 \\
216\end{array}$ & $\begin{array}{r}15 \\
144 \\
594 \\
2558 \\
274\end{array}$ \\
\hline $\begin{array}{l}\text { Average length of } \\
\text { stay per discharge }\end{array}$ & 4.8 & 16.3 & 20.2 & 14.8 & 7.8 & 9.9 \\
\hline
\end{tabular}

2 Only discharges with a length of stay less than 90 days ICD10 160-169

Source: The national in-patient registers 
Morbidity, Medical Treatment, Accidents and Pharmaceutical products

Table 3.4.9 Discharges, patients treated and average length of stay in hospital for chronic obstructive pulmonary disease and bronchiectasis, 2014

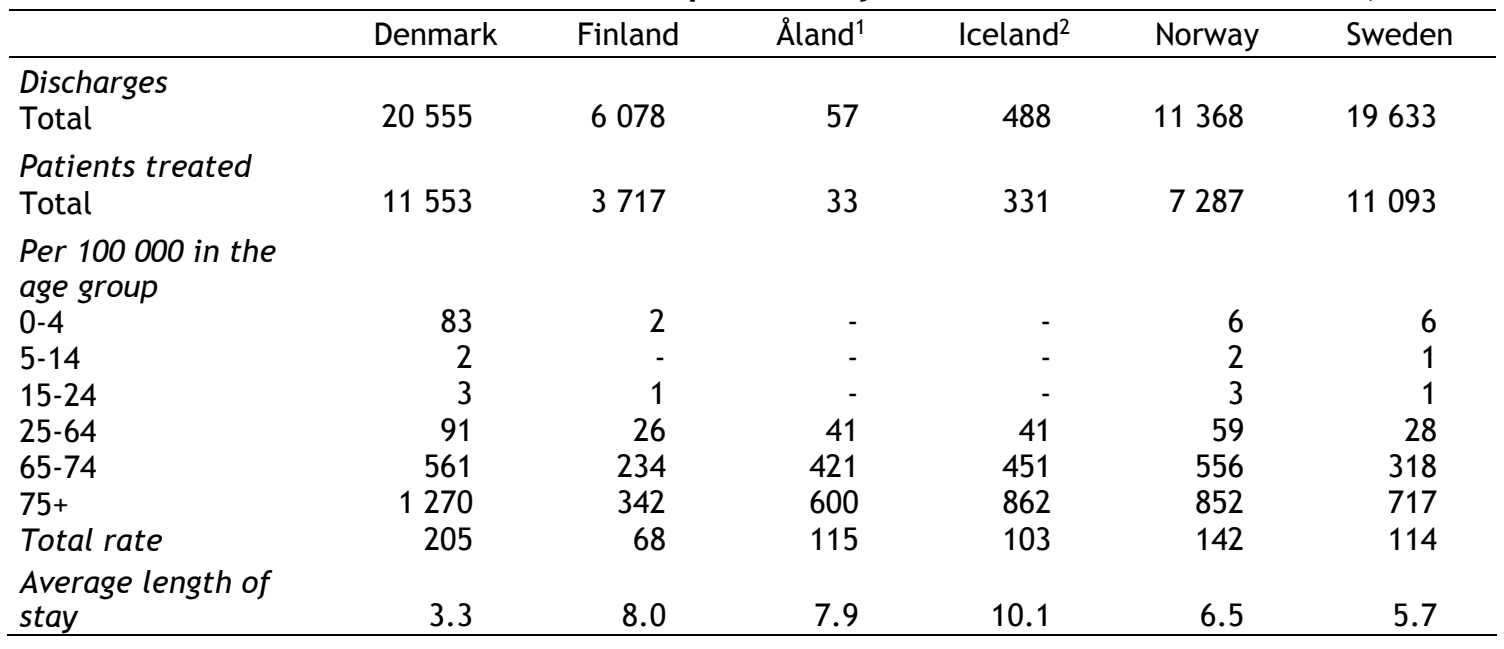

1 Average 2010-14

2 Only discharges with a length of stay less than 90 days

ICD10 J40-J44, J47

Source: The national in-patient registers

Table 3.4.10 Discharges, patients treated and average length of stay in hospital for asthma, 2014

\begin{tabular}{lcccccc}
\hline & Denmark & Finland & Åland $^{1}$ & Iceland $^{2}$ & Norway & Sweden \\
\hline $\begin{array}{l}\text { Discharges } \\
\text { Total }\end{array}$ & 6435 & 3171 & 18 & 59 & 3244 & 5053 \\
$\begin{array}{l}\text { Patients treated } \\
\text { Total }\end{array}$ & 4963 & 2617 & 16 & 55 & 2785 & 4218 \\
$\begin{array}{l}\text { Per } 100 \text { 000 in the } \\
\text { age group }\end{array}$ & & & & & & \\
$0-4$ & 493 & 132 & 347 & 81 & 261 & 369 \\
$5-14$ & 143 & 41 & 70 & 2 & 73 & 46 \\
$\begin{array}{l}15-24 \\
25-64\end{array}$ & 74 & 14 & 25 & 6 & 27 & 14 \\
$65-74$ & 55 & 28 & 24 & 9 & 33 & 16 \\
$75+$ & 38 & 60 & 40 & 27 & 50 & 27 \\
Total rate & 45 & 162 & 142 & 58 & 59 & 61 \\
Average length of & 88 & 48 & 58 & 17 & 54 & 44 \\
stay & 1.8 & 5.3 & 3.5 & 4.6 & 6.1 & 2.2 \\
\hline
\end{tabular}

1 Average 2010-14

2 Only discharges with a length of stay less than 90 days ICD10 J45-J46

Source: The national in-patient registers 
Morbidity, Medical Treatment, Accidents and Pharmaceutical products

Table 3.4.11 Discharges, patients treated and average length of stay in hospital for alcoholic liver disease, 2014

\begin{tabular}{|c|c|c|c|c|c|c|}
\hline & Denmark & Finland & Åland ${ }^{1}$ & Iceland $^{2}$ & Norway & Sweden \\
\hline $\begin{array}{l}\text { Discharges } \\
\text { Men, Total } \\
\text { Women, Total }\end{array}$ & $\begin{array}{r}1997 \\
866\end{array}$ & $\begin{array}{r}1632 \\
658\end{array}$ & $\begin{array}{l}3 \\
1\end{array}$ & $\begin{array}{r}20 \\
4\end{array}$ & $\begin{array}{l}573 \\
266\end{array}$ & $\begin{array}{r}1338 \\
559\end{array}$ \\
\hline $\begin{array}{l}\text { Patients treated } \\
\text { Men, Total } \\
\text { Women, Total }\end{array}$ & $\begin{array}{r}1113 \\
484\end{array}$ & $\begin{array}{l}925 \\
361\end{array}$ & $\begin{array}{l}2 \\
1\end{array}$ & $\begin{array}{r}11 \\
4\end{array}$ & $\begin{array}{l}350 \\
156\end{array}$ & $\begin{array}{l}818 \\
352\end{array}$ \\
\hline $\begin{array}{l}\text { Patients treated pe } \\
100000 \text { men } \\
\text { in the age group } \\
0-44 \\
45-64 \\
65+ \\
\text { Total rate }\end{array}$ & $\begin{array}{r}5 \\
97 \\
72 \\
40\end{array}$ & $\begin{array}{r}6 \\
83 \\
49 \\
34\end{array}$ & $\begin{array}{r}- \\
56 \\
8 \\
17\end{array}$ & $\begin{array}{r}2 \\
18 \\
11 \\
7\end{array}$ & $\begin{array}{l}1 \\
32 \\
32 \\
14\end{array}$ & $\begin{array}{r}1 \\
36 \\
38 \\
17\end{array}$ \\
\hline $\begin{array}{l}\text { Patients treated pe } \\
100000 \text { women } \\
\text { in the age group }\end{array}$ & & & & & & \\
\hline $\begin{array}{l}0-44 \\
45-64 \\
65+ \\
\text { Total rate }\end{array}$ & $\begin{array}{r}2 \\
43 \\
27 \\
17\end{array}$ & $\begin{array}{r}2 \\
32 \\
17 \\
13\end{array}$ & $\begin{array}{r}- \\
5 \\
14 \\
4\end{array}$ & $\begin{array}{l}1 \\
5 \\
5 \\
2\end{array}$ & $\begin{array}{r}1 \\
16 \\
9 \\
6\end{array}$ & $\begin{array}{r}1 \\
15 \\
13 \\
7\end{array}$ \\
\hline $\begin{array}{l}\text { Average length of } \\
\text { stay per discharge }\end{array}$ & 6.6 & 7.5 & 13.1 & 16.7 & 6.9 & 8.3 \\
\hline
\end{tabular}

1 Average 2010-14

2 Only discharges with a length of stay less than 90 days ICD10 K70

Source: The national in-patient registers 
Morbidity, Medical Treatment, Accidents and Pharmaceutical products

Table 3.4.12 Discharges, patients treated and average length of stay in hospital for other diseases of the liver, 2014

\begin{tabular}{|c|c|c|c|c|c|c|}
\hline & Denmark & Finland & Åland ${ }^{1}$ & Iceland $^{2}$ & Norway & Sweden \\
\hline $\begin{array}{l}\text { Discharges } \\
\text { Men, Total } \\
\text { Women, Total }\end{array}$ & $\begin{array}{l}1783 \\
1597\end{array}$ & $\begin{array}{r}998 \\
1047\end{array}$ & $\begin{array}{l}2 \\
3\end{array}$ & $\begin{array}{l}15 \\
32\end{array}$ & $\begin{array}{l}813 \\
874\end{array}$ & $\begin{array}{l}1484 \\
1368\end{array}$ \\
\hline $\begin{array}{l}\text { Patients treated } \\
\text { Men, Total } \\
\text { Women, Total }\end{array}$ & $\begin{array}{r}1095 \\
983\end{array}$ & $\begin{array}{l}675 \\
751\end{array}$ & $\begin{array}{l}2 \\
3\end{array}$ & $\begin{array}{l}14 \\
26\end{array}$ & $\begin{array}{l}549 \\
632\end{array}$ & $\begin{array}{r}1011 \\
953\end{array}$ \\
\hline $\begin{array}{l}\text { Patients treated } \\
100 \text { 000 men } \\
\text { in the age group } \\
0-44 \\
45-64 \\
65+ \\
\text { Total rate }\end{array}$ & $\begin{array}{l}13 \\
70 \\
78 \\
39\end{array}$ & $\begin{array}{r}8 \\
40 \\
59 \\
25\end{array}$ & $\begin{array}{r}8 \\
20 \\
32 \\
14\end{array}$ & $\begin{array}{r}2 \\
15 \\
32 \\
9\end{array}$ & $\begin{array}{r}8 \\
30 \\
59 \\
21\end{array}$ & $\begin{array}{r}6 \\
32 \\
52 \\
21\end{array}$ \\
\hline $\begin{array}{l}\text { Patients treated } \\
100 \text { o00 women } \\
\text { in the age group } \\
0-44 \\
45-64 \\
65+ \\
\text { Total rate }\end{array}$ & $\begin{array}{l}10 \\
55 \\
75 \\
35\end{array}$ & $\begin{array}{l}10 \\
41 \\
51 \\
27\end{array}$ & $\begin{array}{l}11 \\
14 \\
48 \\
20\end{array}$ & $\begin{array}{r}2 \\
18 \\
77 \\
16\end{array}$ & $\begin{array}{r}9 \\
39 \\
56 \\
25\end{array}$ & $\begin{array}{r}7 \\
25 \\
45 \\
20\end{array}$ \\
\hline $\begin{array}{l}\text { Average length of } \\
\text { stay per discharge }\end{array}$ & 5.2 & 6.0 & 7.4 & 9.4 & 6.9 & 7.9 \\
\hline
\end{tabular}

1 Average 2010-14

2 Only discharges with a length of stay less than 90 days ICD10 K71-77

Source: The national in-patient registers 
Table 3.4.13 Discharges, patients treated and average length of stay in hospital for intervertebral disc disorders, 2014

\begin{tabular}{|c|c|c|c|c|c|c|}
\hline & Denmark & Finland & Åland ${ }^{1}$ & Iceland $^{2}$ & Norway & Sweden \\
\hline $\begin{array}{l}\text { Discharges } \\
\text { Men, Total } \\
\text { Women, Total }\end{array}$ & $\begin{array}{l}3507 \\
3383\end{array}$ & $\begin{array}{l}2662 \\
2519\end{array}$ & $\begin{array}{l}4 \\
5\end{array}$ & $\begin{array}{l}50 \\
47\end{array}$ & $\begin{array}{l}3303 \\
2979\end{array}$ & $\begin{array}{l}2712 \\
2577\end{array}$ \\
\hline $\begin{array}{l}\text { Patients treated } \\
\text { Men, Total } \\
\text { Women, Total }\end{array}$ & $\begin{array}{l}2947 \\
2811\end{array}$ & $\begin{array}{l}2303 \\
2133\end{array}$ & $\begin{array}{l}3 \\
4\end{array}$ & $\begin{array}{l}43 \\
43\end{array}$ & $\begin{array}{l}2800 \\
2488\end{array}$ & $\begin{array}{l}2212 \\
2094\end{array}$ \\
\hline $\begin{array}{l}\text { Patients treated per } \\
100000 \text { men } \\
\text { in the age group } \\
0-24 \\
25-44 \\
45-64 \\
65+ \\
\text { Total rate }\end{array}$ & $\begin{array}{r}7 \\
151 \\
184 \\
91 \\
105\end{array}$ & $\begin{array}{r}9 \\
146 \\
132 \\
53 \\
86\end{array}$ & $\begin{array}{r}0 \\
50 \\
30 \\
16 \\
23\end{array}$ & $\begin{array}{r}3 \\
40 \\
38 \\
42 \\
27\end{array}$ & $\begin{array}{r}8 \\
133 \\
207 \\
106 \\
108\end{array}$ & $\begin{array}{l}6 \\
66 \\
78 \\
37 \\
46\end{array}$ \\
\hline $\begin{array}{l}\text { Patients treated per } \\
100000 \text { women } \\
\text { in the age group } \\
0-24 \\
25-44 \\
45-64 \\
65+ \\
\text { Total rate }\end{array}$ & $\begin{array}{r}11 \\
148 \\
166 \\
81 \\
99\end{array}$ & $\begin{array}{r}13 \\
122 \\
119 \\
58 \\
77\end{array}$ & $\begin{array}{l}16 \\
64 \\
29 \\
14 \\
31\end{array}$ & $\begin{array}{r}2 \\
35 \\
56 \\
23 \\
27\end{array}$ & $\begin{array}{r}11 \\
127 \\
178 \\
86 \\
97\end{array}$ & $\begin{array}{r}6 \\
70 \\
66 \\
33 \\
43\end{array}$ \\
\hline $\begin{array}{l}\text { Average length of } \\
\text { stay per discharge } \\
\text { Men } \\
\text { Women }\end{array}$ & $\begin{array}{l}2.4 \\
3.3\end{array}$ & $\begin{array}{l}3.7 \\
4.0\end{array}$ & $\begin{array}{l}5.1 \\
6.2\end{array}$ & $\begin{array}{l}3.4 \\
4.2\end{array}$ & $\begin{array}{l}3.1 \\
3.5\end{array}$ & $\begin{array}{l}3.6 \\
3.8\end{array}$ \\
\hline
\end{tabular}

1 Average 2010-14

2 Only discharges with a length of stay less than 90 days ICD10 M50-51

Source: The national in-patient registers 
Morbidity, Medical Treatment, Accidents and Pharmaceutical products

Table 3.4.14 Discharges, patients treated and average length of stay in hospital for fracture of the femur, 2014

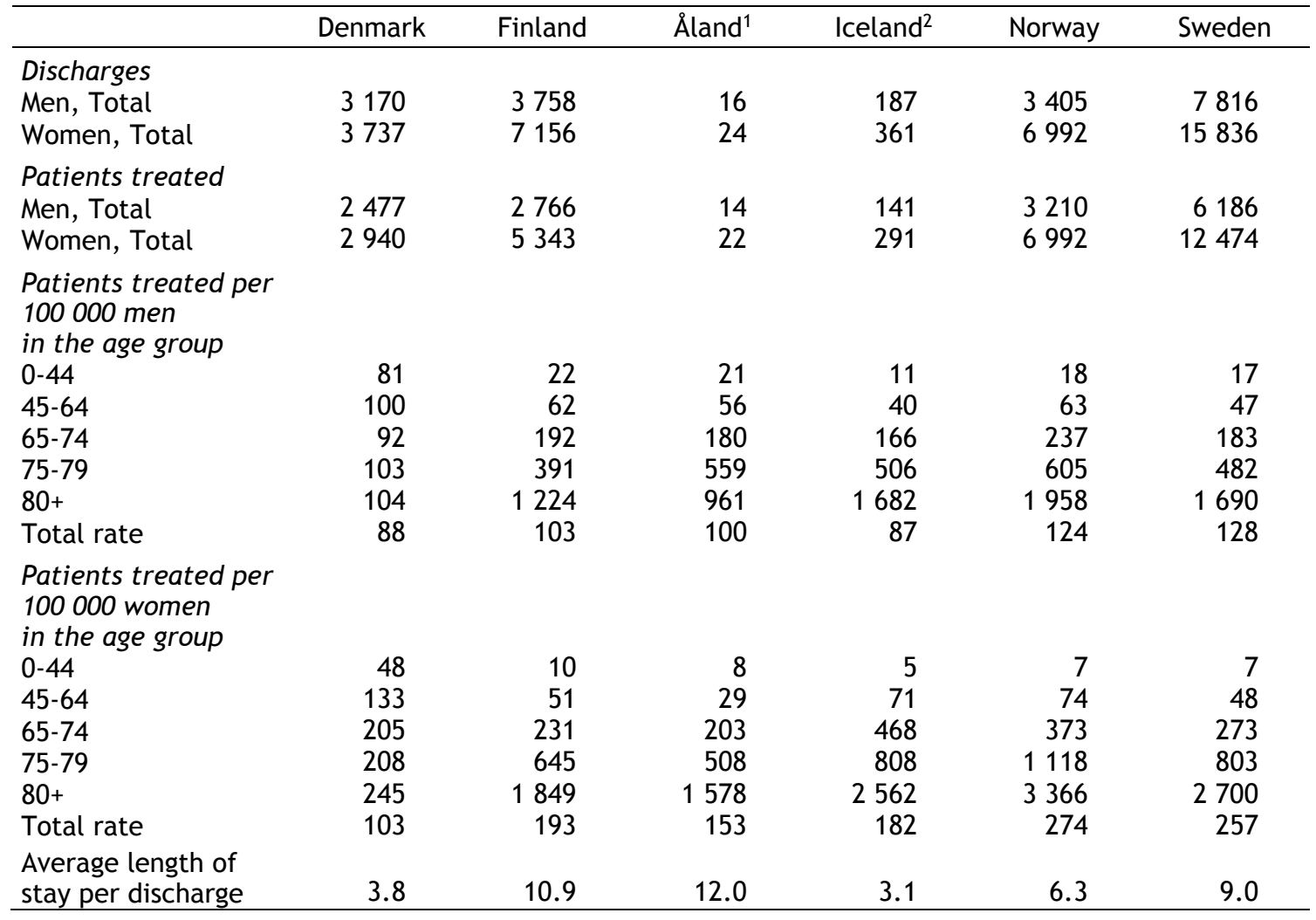

1 Average 2010-14

2 Only discharges with a length of stay less than 90 days

ICD10 S72

Source: The national in-patient registers 
Morbidity, Medical Treatment, Accidents and Pharmaceutical products

Figure 3.4.7 Average bed days for somatic wards, 2000-2014

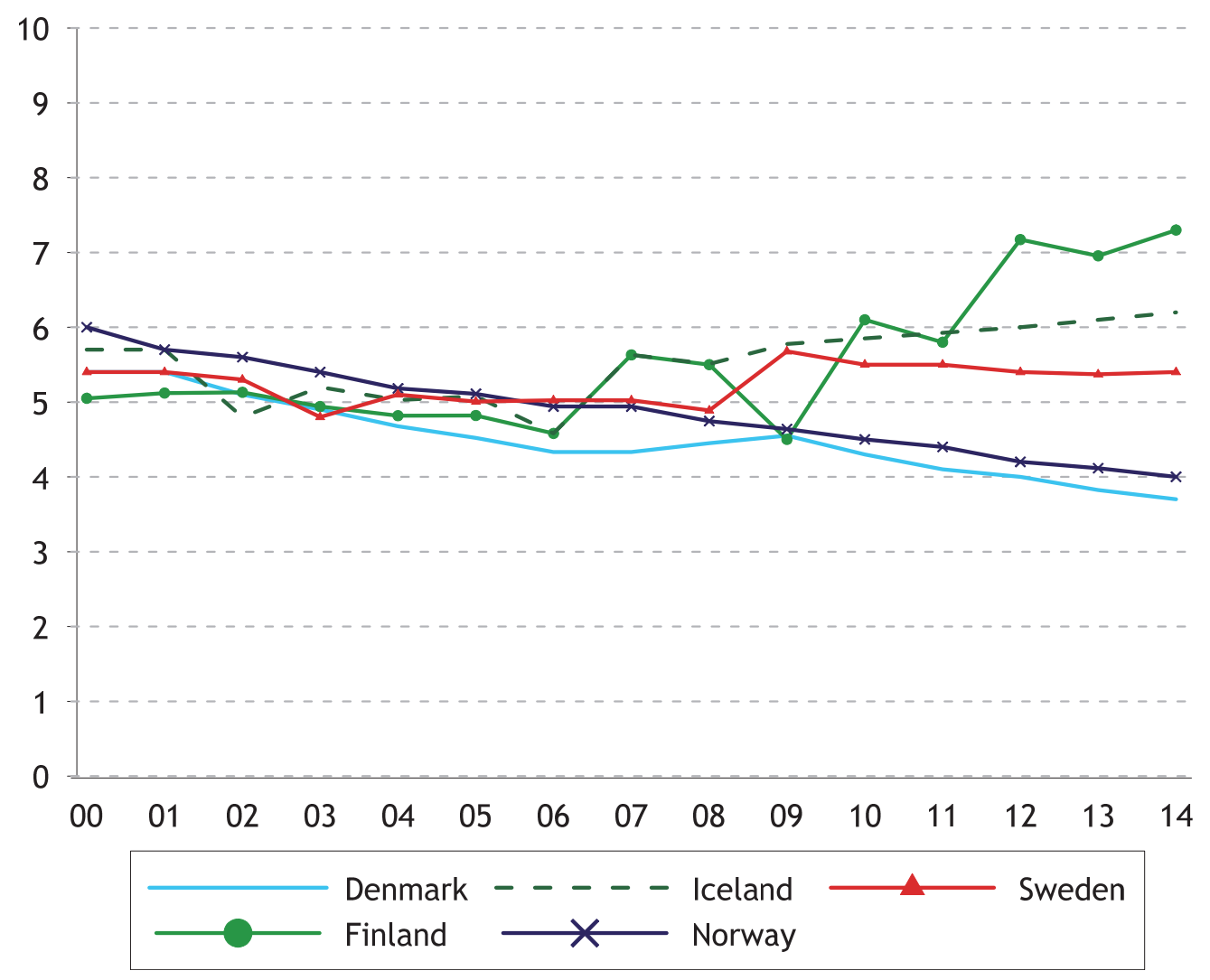


Table 3.4.15 In-patient treatment in psychiatric wards, by age and gender, 2014

\begin{tabular}{|c|c|c|c|c|c|c|c|}
\hline & Denmark & $\begin{array}{l}\text { Faroe } \\
\text { Islands }\end{array}$ & Finland $^{1}$ & Åland ${ }^{1,2}$ & Iceland $^{3}$ & Norway $^{4}$ & Sweden 5 \\
\hline Discharges, total & 51961 & 261 & 37311 & 275 & 2415 & 66252 & 105248 \\
\hline $\begin{array}{l}\text { Discharges per } \\
1000 \text { inhabitants }\end{array}$ & 9.2 & 5.4 & 6.8 & 9.5 & 7.4 & 12.9 & 10.9 \\
\hline Total bed days & 790466 & 11523 & 1301332 & 5385 & 26333 & 1390750 & 1438492 \\
\hline $\begin{array}{l}\text { Bed days per } \\
1000 \text { inhabitants }\end{array}$ & 140.2 & 239 & 238.3 & 186.8 & 80.4 & 270.7 & 148.4 \\
\hline $\begin{array}{l}\text { Treated patients, } \\
\text { total }\end{array}$ & 26143 & 285 & 23966 & 151 & 1546 & 36316 & 51998 \\
\hline \multicolumn{8}{|l|}{$\begin{array}{l}\text { Treated patients, } \\
\text { per } 1000\end{array}$} \\
\hline \multicolumn{8}{|l|}{ Men } \\
\hline $0-14$ & 0.4 & - & 2.2 & 0.2 & 1.2 & 0.9 & 0.2 \\
\hline $15-29$ & 6.0 & 0.5 & 6.1 & 7.9 & 7.7 & 9.2 & 7.6 \\
\hline $30-44$ & 7.0 & 1.0 & 6.0 & 6.3 & 6.9 & 11.3 & 7.3 \\
\hline $45-64$ & 5.8 & 1.0 & 4.4 & 7.5 & 4.3 & 8.7 & 7.9 \\
\hline $65-79$ & 3.1 & 0.3 & 3.0 & 4.1 & 2.9 & 4.1 & 4.5 \\
\hline $80+$ & 3.6 & 0.0 & 2.5 & 2.1 & 1.2 & 4.3 & 3.6 \\
\hline Total & 4.7 & 2.8 & 4.4 & 5.3 & 4.7 & 7.3 & 5.7 \\
\hline \multicolumn{8}{|l|}{ Women } \\
\hline $0-14$ & 0.6 & 0.1 & 1.7 & 0.2 & 1.0 & 0.8 & 0.5 \\
\hline $15-29$ & 7.0 & 1.9 & 7.6 & 9.9 & 8.2 & 10.8 & 8.4 \\
\hline $30-44$ & 5.6 & 0.9 & 5.0 & 5.8 & 5.7 & 8.6 & 6.4 \\
\hline $45-64$ & 6.8 & 2.1 & 4.2 & 5.8 & 5.1 & 8.2 & 5.8 \\
\hline $65-79$ & 3.0 & 0.4 & 3.9 & 4.8 & 3.5 & 5.3 & 4.1 \\
\hline $80+$ & 4.1 & 0.0 & 3.4 & 2.3 & 1.7 & 4.4 & 3.6 \\
\hline Total & 4.7 & 5.3 & 4.4 & 5.1 & 4.7 & 6.9 & 5.1 \\
\hline \multicolumn{8}{|l|}{ Men and women } \\
\hline $0-14$ & 0.5 & 0.1 & 1.9 & 0.2 & 1.1 & 0.8 & 0.3 \\
\hline $15-29$ & 6.5 & 1.5 & 6.8 & 8.9 & 7.9 & 10.0 & 8.0 \\
\hline $30-44$ & 6.3 & 1.4 & 5.5 & 6.0 & 6.3 & 10.0 & 6.8 \\
\hline $45-64$ & 6.3 & 2.0 & 4.3 & 6.6 & 4.7 & 8.4 & 6.9 \\
\hline $65-79$ & 3.0 & 0.5 & 3.5 & 4.5 & 3.2 & 4.7 & 4.3 \\
\hline $80+$ & 3.9 & 0.0 & 3.1 & 2.2 & 1.5 & 4.3 & 3.6 \\
\hline Total & 4.7 & 5.4 & 4.4 & 5.2 & 4.7 & 7.1 & 5.4 \\
\hline $\begin{array}{l}\text { Average length of stay } \\
\text { per discharge }\end{array}$ & 15.2 & 44.0 & 34.9 & 19.6 & 10.9 & 21.0 & 13.7 \\
\hline
\end{tabular}

1 Figures for psychiatry include activity in psychiatric hospitals. Beds for adults and children are included. Figures for somatic wards include activity in somatic hospitals. A patient is only counted once in somatic and/or psychiatric wards

2 Average 2010-14

3 Only discharges with a length of stay less than 90 days

4 Figures for psychiatry include activity in psychiatric hospitals, psychiatric wards and community mental health care centre. Beds for adults, children and people receiving treatment for addiction are included. Figures for somatic wards include activity in somatic hospitals (not rehabilitation). A patient is only counted once in somatic and/or psychiatric wards

5 Figures for psychiatry include activity in psychiatric wards in ordinary and specialized hospitals. Figures for somatic wards include activities in all other wards in ordinary and specialized hospitals. Beds for adults and children regardless of treatment are included in both figures. A patient is only counted once in somatic and/or psychiatric wards

Source: The national in-patient registers 
Table 3.4.16 Discharges from hospitals ${ }^{1}$ per 1000 inhabitants, by age and gender, 2014

\begin{tabular}{lrrrrrr}
\hline Age & Denmark & Finland & Áland $^{2}$ & Iceland $^{3}$ & Norway & Sweden \\
\hline Men & & & & & & \\
$0-14$ & 206 & 79 & 75 & 60 & 85 & 134 \\
$15-44$ & 108 & 56 & 49 & 26 & 69 & 61 \\
$45-64$ & 245 & 139 & 112 & 78 & 164 & 135 \\
$65-69$ & 428 & 264 & 235 & 182 & 312 & 249 \\
$70-74$ & 541 & 354 & 318 & 267 & 414 & 333 \\
$75-79$ & 684 & 458 & 395 & 361 & 526 & 449 \\
$80+$ & 964 & 630 & 707 & 559 & 728 & 703 \\
Total & 246 & 141 & 131 & 81 & 153 & 157 \\
Women & & & & & & \\
$0-14$ & 181 & 63 & 63 & 51 & 54 & 123 \\
$15-44$ & 215 & 133 & 142 & 117 & 164 & 151 \\
$45-64$ & 218 & 119 & 122 & 85 & 169 & 120 \\
$65-69$ & 327 & 197 & 195 & 177 & 233 & 196 \\
$70-74$ & 416 & 265 & 261 & 266 & 305 & 267 \\
$75-79$ & 553 & 348 & 356 & 315 & 404 & 362 \\
$80+$ & 777 & 516 & 625 & 459 & 3621 & 583 \\
Total & 268 & 163 & 171 & 122 & 119 & 184 \\
\hline
\end{tabular}

1 Includes somatic wards in regular hospitals and in somatic special hospitals

2 Average 2010-14

3 Only discharges with a length of stay less than 90 days

Source: The national in-patient registers

Table 3.4.17 Bed days for hospitals ${ }^{1}$ per 1000 inhabitants, by age and gender, 2014

\begin{tabular}{lrrrrrr}
\hline Age & Denmark & Finland & Åland ${ }^{2}$ & Iceland $^{3}$ & Norway & Sweden \\
\hline Men & & & & & & \\
$0-14$ & 480 & 392 & 285 & 214 & 300 & 793 \\
$15-44$ & 489 & 452 & 293 & 118 & 214 & 345 \\
$45-64$ & 952 & 905 & 787 & 498 & 657 & 702 \\
$65-69$ & 1685 & 1728 & 1670 & 1383 & 1448 & 1272 \\
$70-74$ & 2147 & 2524 & 2312 & 2128 & 2004 & 1742 \\
$75-79$ & 2813 & 3566 & 3139 & 3350 & 2651 & 2559 \\
$80+$ & 4036 & 6045 & 7578 & 6319 & 3714 & 4444 \\
Total & 942 & 1023 & 974 & 571 & 642 & 881 \\
Women & & & & & & 735 \\
$0-14$ & 452 & 308 & 238 & 164 & 251 & 542 \\
$15-44$ & 681 & 694 & 723 & 267 & 460 & 586 \\
$45-64$ & 834 & 744 & 958 & 336 & 582 & 139 \\
$65-69$ & 1315 & 1316 & 1320 & 1095 & 120 & 1575 \\
$70-74$ & 1689 & 1891 & 1884 & 1726 & 1545 & 2136 \\
$75-79$ & 2358 & 2949 & 2915 & 2512 & 1977 & 3914 \\
$80+$ & 3349 & 6346 & 8477 & 5966 & 2754 & 948 \\
Total & 971 & 1208 & 1384 & 55 & 701 & \\
\hline
\end{tabular}

1 Includes somatic wards in regular hospitals and in somatic special hospitals

2 Average 2010-14

3 Only discharges with a length of stay less than 90 days

Source: The national in-patient registers 
Morbidity, Medical Treatment, Accidents and Pharmaceutical products

Figure 3.4.8 Bed days for hospitals ${ }^{1}$ per 1000 inhabitants, by age and gender, 2014
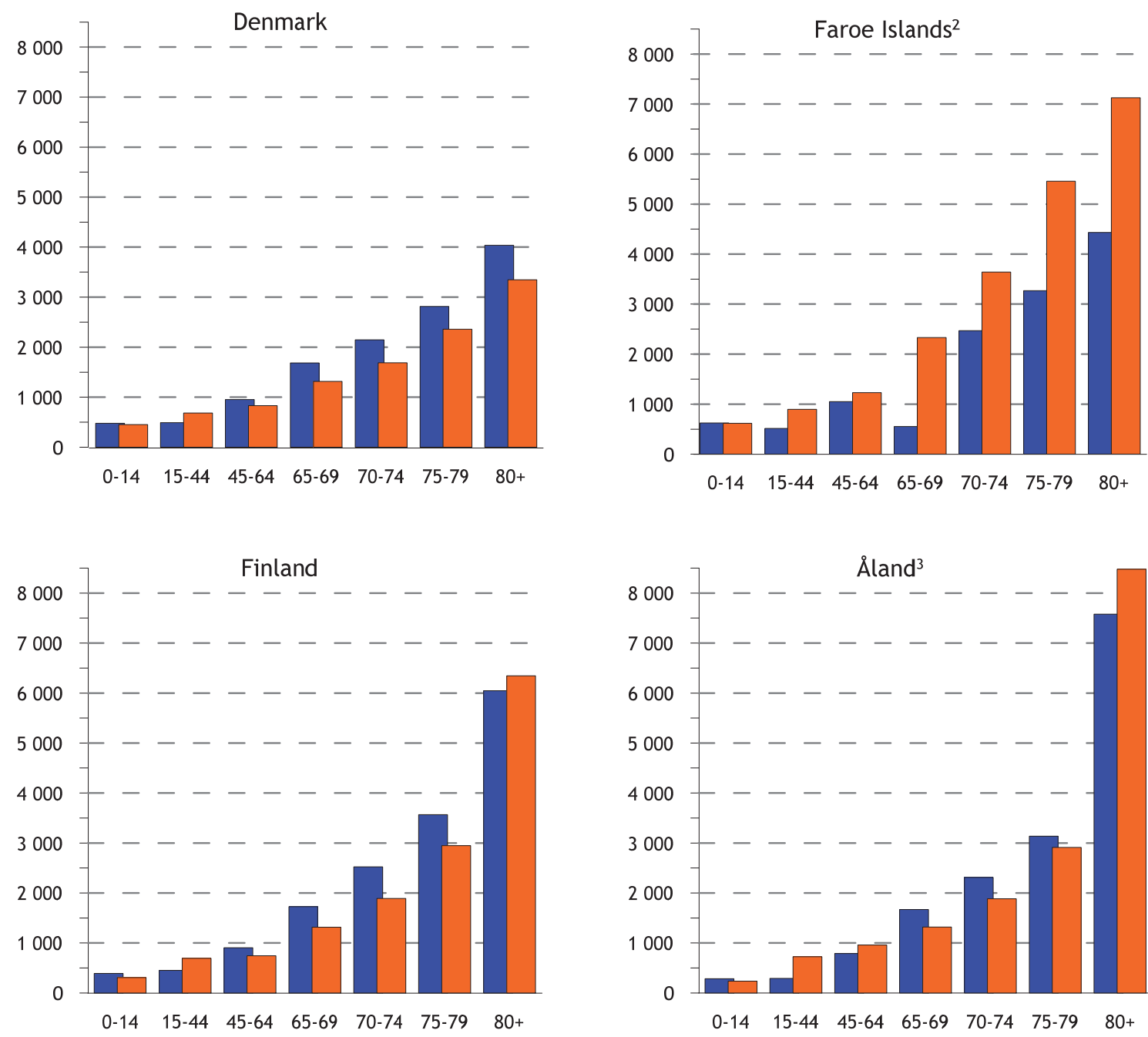

Men

Women

Continues 
Figure 3.4.8 Bed days for hospitals ${ }^{1}$ per 1000 inhabitants, by age and gender, 2014, continued
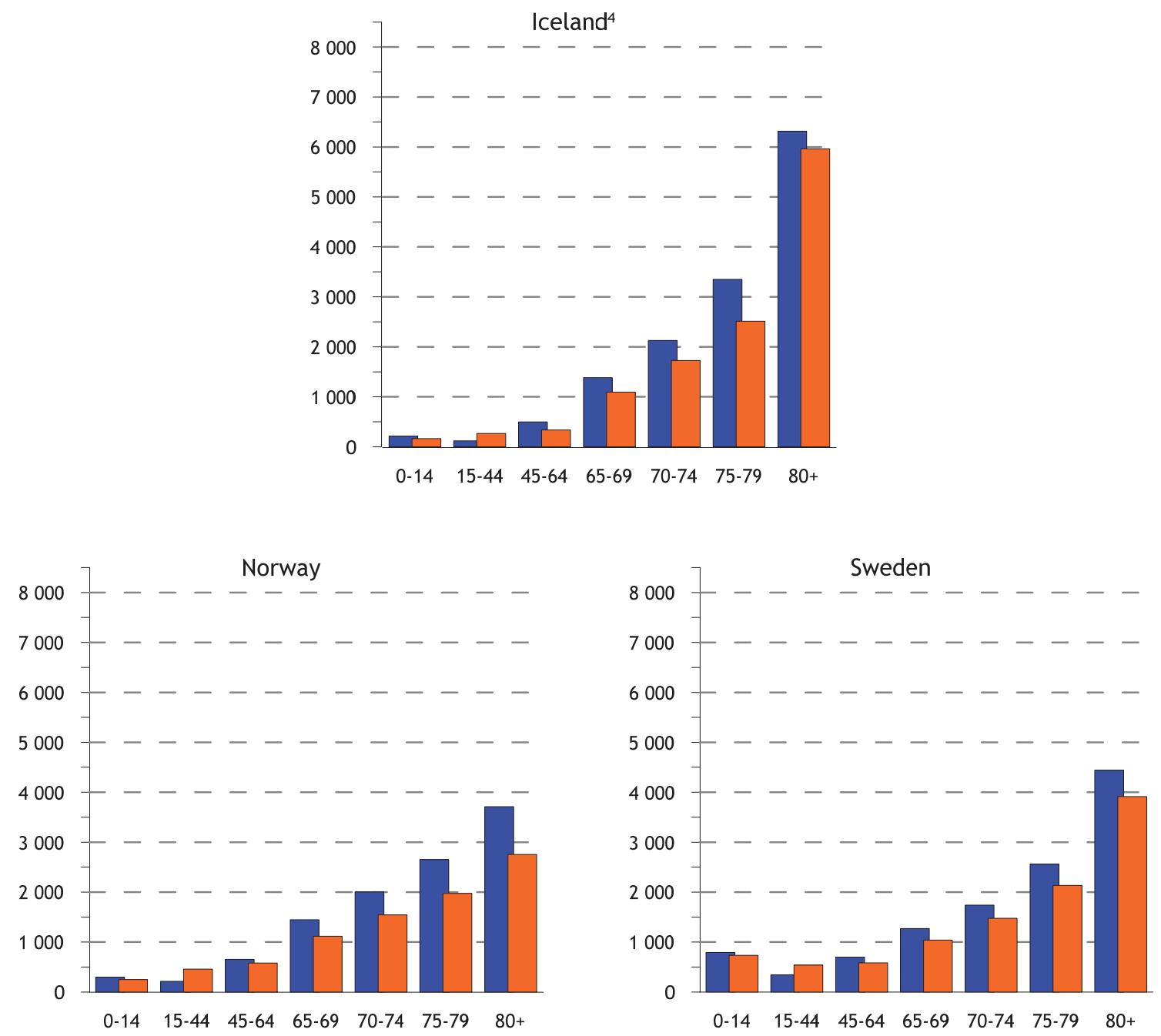

\section{Men}

Women

1 Includes somatic wards in regular hospitals and in somatic special hospitals

22013

3 Average 2010-14

4 Only discharges with a length of stay less than 90 days

Source: The national in-patient registers 


\subsection{Surgical procedures}

In this section, data on selected surgical procedures performed in short-term somatic hospitals are presented. The selected list of procedures used here was developed for international comparison by the EU Hospital Data Project (HDP2).

The HDP2 list consists of 30 selected procedures or procedure groups (with six subgroups) within a broad range of medical specialities. Several criteria were combined for the selection of procedures, such as how common a procedure is, its potential for day surgery, changing technique over time, cost, public health importance and continuity with existing statistics. The complete list with definitions of the procedures, the main reasons for selection of the different procedures and some caveats for the interpretation of the statistics is presented in one document (See link HDP2 list of procedures at the start of the chapter). All the procedures are also defined with codes from the NOMESCO Classification of Surgical Procedures (NCSP-E), which is the common English language version of the NCSP.

\section{Outline of this section}

The presentation starts with two summary tables (Table 3.5.1a+b) showing the number per 100000 inhabitants for each procedure on the selected list, performed on male and female in-patients. Laparoscopic techniques are increasingly being used for five procedures on the list. Table 3.5.3 shows the proportions of these that are performed laparoscopically and also the relative frequency of secondary hip replacements. Eight of the procedures on the list that are often performed as day surgery are presented in Table 3.5.2, which shows the proportion of the total number of these procedures that are carried out as day surgery. Two figures (Figures 3.5.1 and 3.5.2) show the development over time for three common procedures.

Finally, in a series of tables (3.5.4 - 3.5.17) data on some of the procedures are presented in greater detail, showing the number of operations and population rates with age distributions for males and females, similar to the statistics presented in earlier editions of Health Statistics. These tables show the total number of procedures that are reported, both in-patient surgery and day surgery taken together.

\section{Quality and limitations of the data}

In its annual report in 2002, NOMESCO presented a theme section dealing with validity and comparability of Nordic hospital statistics on surgical procedures, and in 2003, a corresponding report on day surgery statistics. Based on the recommendations of these studies, some changes were made in the reporting procedure, aiming at improving comparability. In its report, the EU Hospital Data Project (HDP2) also presented a thorough analysis of the methodological difficulties involved in achieving valid and comparable data on hospital procedures.

How procedures should be counted is one of the problems. In the Nordic countries, there is no common concept such as a principal procedure, if more than one procedure is performed during the same hospital stay (corresponding to a main diagnosis as the basis for diagnosis-related statistics). Procedure statistics are therefore based on any procedure registered during a hospital stay and reported to the national patient register. This could result in a hospital stay being counted twice, if more than one 
procedure on the list is performed during the same stay, e.g. a colonoscopy that is followed by a colectomy. Since both are on the selected list, both will be counted.

The fact that the Nordic countries use the same procedure classification makes comparisons easier. The relevant NCSP-E codes for each procedure are listed in all tables.

In order to describe surgical activities in hospitals, it is necessary to include both in-patient surgery and day surgery, which constitutes an increasing part. The HDP2 list includes both procedures mainly performed on in-patients and procedures often performed as day surgery. Formal definitions of day treatment and day surgery differ somewhat between countries. Day treatment involves patients who are formally admitted to the hospital for examination or treatment and discharged the same day. Without exact definitions of day treatment, it may be necessary to approximate and count as day treatment all stays for which the date of admission and the date of discharge are the same. However, some of these stays may refer to patients who were transferred to another hospital or who died, and thus are not day patients in a real sense. There is also a blurred border between day treatment and out-patient treatment provided at the hospital. Furthermore, some of the procedures on the list are also performed outside hospitals in specialist centres and private clinics and these may not be reported to the national patient registers.

These difficulties are reflected in the Nordic statistics. While Iceland has not been able to report on day surgery at all for 2014, Denmark has had some difficulties in separating day treatment and out-patient treatment. Known under-reporting in the national patient registers is also caused by some private hospitals not reporting centrally.

Thus organizational differences may influence the reporting. There are also different rules for reporting to national registers, e.g. in Finland where reporting of minor procedures, such as diagnostic colonoscopy, is not necessary. Some of these problems are reflected in the caveats in the HDP2 list.

Table 3.5.1 shows the rates per 100000 inhabitants for men and women for all surgical procedures on the new list. However, it only covers hospitalized patients and therefore does not give a complete picture of surgical procedures that are often performed on an out-patient basis, e.g. cataract surgery, colonoscopy and hernia surgery. Several of the more common surgical procedures that are performed on inpatients, tend to show almost the same rates in all countries (with the exception of Åland, which has a small number of inhabitants). These are, for example, transluminal coronary angioplasty and hysterectomy. The difference between the genders are already known in all the countries, where the numbers are higher for men for heart surgery and hernia operations, and higher for women for thyroidectomy, cholecystectomy and replacement of the hip joint. The low rates for decompression of bone marrow and nerve roots in Sweden are to some degree due to lack of reporting from three private special hospitals. Strikingly high rates are seen for hernia operations for men in Finland and for cholecystectomy for women in Iceland. 
Table 3.5.1a Surgical procedures performed on in-patients per 100000 inhabitants by list of selected procedures, men ${ }^{1}$

\begin{tabular}{|c|c|c|c|c|c|c|}
\hline $\begin{array}{l}\text { Surgical procedures } \\
\text { (NCSP-E codes in brackets) }\end{array}$ & $\begin{array}{l}\text { Denmark } \\
2014\end{array}$ & $\begin{array}{c}\text { Finland } \\
2014\end{array}$ & $\begin{array}{c}\text { Åland } \\
2010-14\end{array}$ & $\begin{array}{l}\text { Iceland } \\
2014\end{array}$ & $\begin{array}{l}\text { Norway } \\
2014\end{array}$ & $\begin{array}{c}\text { Sweden } \\
2014\end{array}$ \\
\hline $\begin{array}{l}\text { 1: Extirpation, excision and } \\
\text { destruction of intra-cranial } \\
\text { lesion (AAB00-AAB20, AAB99) }\end{array}$ & 24.1 & 19.9 & 16.9 & 9.3 & 17.9 & 17.9 \\
\hline $\begin{array}{l}\text { 2: Evacuation of subdural } \\
\text { haematoma and intra-cranial } \\
\text { haemorrhage (AAB30, AAD05- } \\
\text { AAD15) }\end{array}$ & 23.8 & 31.9 & 40.9 & 19.8 & 20.5 & 23.6 \\
\hline 3: Discectomy ( $A B C)$ & 163.9 & 142.8 & 73.4 & 63.2 & 139.6 & 71.0 \\
\hline $\begin{array}{l}\text { 4: Thyroidectomy } \\
\text { (BAA20-BAA60) }\end{array}$ & 19.3 & 14.6 & 9.9 & 9.9 & 13.7 & 12.5 \\
\hline $\begin{array}{l}\text { 5: Cataract surgery } \\
\text { (CJC, CJD, CJE, CJF) }\end{array}$ & 10.0 & 20.6 & 26.8 & 5.0 & 18.0 & 19.8 \\
\hline $\begin{array}{l}\text { 6: Cochlear implantation } \\
\text { (DFE00) }\end{array}$ & 5.4 & 2.7 & 2.8 & 1.9 & 1.6 & 3.6 \\
\hline $\begin{array}{l}\text { 7: Tonsillectomy (EMB10- } \\
\text { EMB20) }\end{array}$ & 57.5 & 33.5 & 107.3 & 21.1 & 63.1 & 46.1 \\
\hline $\begin{array}{l}\text { 8: Pulmectomy } \\
\text { (GDB20-21, GDC, GDD) }\end{array}$ & 17.3 & 11.1 & 5.6 & 18.6 & 12.0 & 8.3 \\
\hline $\begin{array}{l}\text { 9: Diagnostic bronchoscopy } \\
\text { with or without biopsy (UGC) }\end{array}$ & 90.3 & 52.6 & 16.9 & 61.4 & 107.0 & 40.0 \\
\hline $\begin{array}{l}\text { 10: Transluminal coronary } \\
\text { angioplasty (FNG02, FNG05) }\end{array}$ & 174.7 & 259.5 & 18.4 & - & 339.6 & 273.6 \\
\hline $\begin{array}{l}\text { 11: Coronary artery bypass } \\
\text { graft (FNC, FND, FNE) }\end{array}$ & 58.8 & 45.3 & 4.2 & 69.4 & 62.0 & 49.2 \\
\hline $\begin{array}{l}\text { 12: Carotid endarterectomy } \\
\text { (PAF20-PAF22) }\end{array}$ & 11.6 & 17.2 & 1.4 & 6.2 & 11.8 & 13.1 \\
\hline $\begin{array}{l}\text { 13: Infrarenal aortic aneurysm } \\
\text { repair (PDG10-PDG24, PDQ10) }\end{array}$ & 23.9 & 16.9 & 11.3 & 9.3 & 25.8 & 11.9 \\
\hline $\begin{array}{l}\text { 14: Femoropopliteal bypass } \\
\text { (PEH) }\end{array}$ & 9.3 & 14.8 & 18.4 & 5.0 & 5.1 & 4.8 \\
\hline $\begin{array}{l}\text { 15: Stem cell transplantation } \\
\left.\text { (not included }^{3}\right)\end{array}$ & 8.2 & 4.7 & - & - & - & - \\
\hline $\begin{array}{l}\text { 16: Colonoscopy with or } \\
\text { without biopsy (JFA15, UJF32, } \\
\text { UJF35, UJF42, UJF45) }\end{array}$ & 286.1 & 59.8 & 33.9 & 106.6 & 197.4 & 123.1 \\
\hline $\begin{array}{l}\text { 17: Colectomy } \\
\text { (JFB20-JFB64, JFH) } \\
\text { Of which: }\end{array}$ & 82.7 & 62.6 & 72.0 & 49.0 & 75.7 & 120.7 \\
\hline $\begin{array}{l}\text { 17A: Laparoscopic colectomy } \\
\text { (JFB21, JFB31, JFB34, JFB41, } \\
\text { JFB44, JFB47, JFB51, JFB61, } \\
\text { JFB64, JFH01, JFH11) }\end{array}$ & 39.0 & 21.2 & 2.8 & 15.5 & 28.0 & 9.5 \\
\hline
\end{tabular}


Table 3.5.1a Surgical procedures performed on in-patients per 100000 inhabitants by list of selected procedure, men, Continued ${ }^{1}$

\begin{tabular}{|c|c|c|c|c|c|c|}
\hline $\begin{array}{l}\text { Surgical procedures } \\
\text { (NCSP-E codes in brackets) }\end{array}$ & $\begin{array}{c}\text { Denmark } \\
2014\end{array}$ & $\begin{array}{c}\text { Finland } \\
2014\end{array}$ & $\begin{array}{l}\text { Åland } \\
\text { 2010-14 }\end{array}$ & $\begin{array}{l}\text { Iceland } \\
2014\end{array}$ & $\begin{array}{c}\text { Norway } \\
2014\end{array}$ & $\begin{array}{c}\text { Sweden } \\
2014\end{array}$ \\
\hline 18: Appendectomy (JEA) & 103.8 & 125.1 & 162.3 & 146.9 & 123.8 & 130.1 \\
\hline $\begin{array}{l}\text { Of which: } \\
\text { 18A: Laparoscopic } \\
\text { appendectomy (JEA01) }\end{array}$ & 93.5 & 48.3 & 8.5 & 91.7 & 103.7 & 65.0 \\
\hline $\begin{array}{l}\text { 19: Cholecystectomy (JKA20, } \\
\text { JKA21) }\end{array}$ & 53.3 & 84.4 & 103.0 & 73.8 & 47.7 & 79.9 \\
\hline $\begin{array}{l}\text { Of which: } \\
\text { 19A: Laparoscopic } \\
\text { cholecystectomy (JKA21) }\end{array}$ & 41.9 & 65.1 & 76.2 & 62.6 & 42.1 & 61.2 \\
\hline $\begin{array}{l}\text { 20: Repair of inguinal hernia } \\
\text { (JAB) } \\
\text { Of which: }\end{array}$ & 64.1 & 123.0 & 141.2 & 39.7 & 78.9 & 58.8 \\
\hline $\begin{array}{l}\text { 20: Laparoscopic repair of } \\
\text { inguinal hernia (JAB11, JAB97) }\end{array}$ & 25.8 & 13.5 & 22.6 & 9.3 & 29.8 & 1.2 \\
\hline $\begin{array}{l}\text { 21: Transplantation of kidney } \\
\text { (KASO0-KAS20) }\end{array}$ & 5.7 & 5.5 & 9.9 & 4.3 & 7.5 & 5.9 \\
\hline $\begin{array}{l}\text { 22: Open prostatectomy (KEC, } \\
\text { KED00, KED96) }\end{array}$ & 41.0 & 44.2 & 69.2 & 27.9 & 68.3 & 67.9 \\
\hline $\begin{array}{l}\text { 23: Transurethral } \\
\text { prostatectomy (KED22, KED52- } \\
\text { KED72, KED98) }\end{array}$ & 90.3 & 131.2 & 162.3 & 88.6 & 129.4 & 100.9 \\
\hline \multicolumn{6}{|l|}{ Of which: } & . \\
\hline $\begin{array}{l}\text { 24A: Laparocopic hysterectomy } \\
\text { (LCC01, LCC11, LCC97, LCD01, } \\
\text { LCD04, LCD11, LCD31, LCD40, } \\
\text { LCD97) }\end{array}$ & . & . & . & . & . & $\cdot$ \\
\hline 25: Caesarean section (MCA) & $\cdot$ & . & . & . & . & . \\
\hline $\begin{array}{l}\text { 26: Arthroscopic excision of } \\
\text { meniscus of knee (NGD01, } \\
\text { NGD11) }\end{array}$ & 5.4 & 8.8 & 11.3 & 0.6 & 16.6 & 3.4 \\
\hline 27: Hip replacement (NFB, NFC) & 187.2 & 201.7 & 184.9 & 153.7 & 168.1 & 166.0 \\
\hline $\begin{array}{l}\text { Of which: } \\
\text { 27A: Secondary hip } \\
\text { replacement (NFC) }\end{array}$ & 21.5 & 29.3 & 14.1 & 25.4 & 19.9 & 17.8 \\
\hline $\begin{array}{l}\text { 28: Total knee re-placement } \\
\text { (NGB20-NGB40) }\end{array}$ & 108.8 & 126.7 & 138.3 & 16.1 & 76.4 & 89.2 \\
\hline $\begin{array}{l}\text { 29: Partial excision of } \\
\text { mammary gland (HAB00, } \\
\text { HAB30, HAB40, HAB99) }\end{array}$ & 0.3 & 0.9 & - & 0.6 & 0.1 & 0.5 \\
\hline $\begin{array}{l}\text { 30: Total mastectomy (HAC10- } \\
\text { HAC25, HAC99) }\end{array}$ & 4.7 & 2.2 & 1.4 & 3.1 & 1.5 & 1.7 \\
\hline $\begin{array}{l}1 \text { The NCSP codes refer to th } \\
\text { NOMESCO 83:2008 } \\
2 \text { In Åland aorta coronary by } \\
\text { to Sweden for these proce } \\
3 \text { Not included in NCSP-E bu }\end{array}$ & $\begin{array}{l}\text { NOMESCC } \\
\text { ass operat } \\
\text { res, and }\end{array}$ & $\begin{array}{l}\text { assificat } \\
\text { are not } \\
\text { treatme }\end{array}$ & $\begin{array}{l}\text { f Surgica } \\
\text { ormed. I } \\
\text { not regis }\end{array}$ & $\begin{array}{l}\text { osedures } \\
\text { ost case } \\
\text { in Åla }\end{array}$ & SP-E-ver & $\begin{array}{l}1.13: 2009 \\
\text { ansferred }\end{array}$ \\
\hline $\begin{array}{l}3 \text { Not included in NCSP-E bu } \\
\text { Source: The national in-patie }\end{array}$ & $\begin{array}{l}n \text { be de } \\
\text { egisters }\end{array}$ & roug & & I natic & assifi & \\
\hline
\end{tabular}


Table 3.5.1b Surgical procedures performed on in-patients per 100000 inhabitants by list of selected procedures, women ${ }^{1}$

\begin{tabular}{|c|c|c|c|c|c|c|}
\hline $\begin{array}{l}\text { Surgical procedures } \\
\text { (NCSP-E codes in brackets) }\end{array}$ & $\begin{array}{c}\text { Denmark } \\
2014\end{array}$ & $\begin{array}{c}\text { Finland } \\
2014\end{array}$ & $\begin{array}{l}\text { Åland } \\
\text { 2010-14 }\end{array}$ & $\begin{array}{c}\text { Iceland } \\
2014\end{array}$ & $\begin{array}{c}\text { Norway } \\
2014\end{array}$ & $\begin{array}{c}\text { Sweden } \\
2014\end{array}$ \\
\hline $\begin{array}{l}\text { 1: Extirpation, excision and } \\
\text { destruction of intra-cranial } \\
\text { lesion (AAB00-AAB20, AAB99) }\end{array}$ & 24.4 & 23.1 & 22.5 & 16.2 & 17.7 & 18.9 \\
\hline $\begin{array}{l}\text { 2: Evacuation of subdural } \\
\text { haematoma and intra-cranial } \\
\text { haemorrhage (AAB30, AAD05- } \\
\text { AAD15) }\end{array}$ & 11.1 & 14.8 & 11.2 & 6.2 & 9.3 & 9.4 \\
\hline 3: Discectomy ( $\mathrm{ABC}$ ) & 149.8 & 129.9 & 96.8 & 74.3 & 137.5 & 74.1 \\
\hline $\begin{array}{l}\text { 4: Thyroidectomy } \\
\text { (BAA20-BAA60) }\end{array}$ & 68.1 & 69.9 & 66.0 & 40.0 & 48.3 & 49.7 \\
\hline $\begin{array}{l}\text { 5: Cataract surgery } \\
\text { (CJC, CJD, CJE, CJF) }\end{array}$ & 12.9 & 22.8 & 40.7 & 1.2 & 17.9 & 18.3 \\
\hline $\begin{array}{l}\text { 6: Cochlear implantation } \\
\text { (DFE00) }\end{array}$ & 5.0 & 2.8 & 2.8 & 3.7 & 1.8 & 3.7 \\
\hline $\begin{array}{l}\text { 7: Tonsillectomy (EMB10- } \\
\text { EMB20) }\end{array}$ & 62.2 & 34.1 & 109.5 & 12.5 & 66.7 & 43.4 \\
\hline $\begin{array}{l}\text { 8: Pulmectomy } \\
\text { (GDB20-21, GDC, GDD) }\end{array}$ & 17.3 & 7.8 & 2.8 & 16.9 & 11.4 & 10.0 \\
\hline $\begin{array}{l}\text { 9: Diagnostic bronchoscopy } \\
\text { with or without biopsy (UGC) }\end{array}$ & 52.5 & 32.6 & 14.0 & 58.1 & 70.4 & 30.8 \\
\hline $\begin{array}{l}\text { 10: Transluminal coronary } \\
\text { angioplasty (FNG02, FNG05) }\end{array}$ & 60.9 & 100.9 & 8.4 & 0.0 & 106.1 & 100.5 \\
\hline $\begin{array}{l}\text { 11: Coronary artery bypass } \\
\text { graft (FNC, FND, FNE) }\end{array}$ & 12.9 & 12.0 & 1.4 & 11.9 & 14.8 & 11.6 \\
\hline $\begin{array}{l}\text { 12: Carotid endarterectomy } \\
\text { (PAF20-PAF22) }\end{array}$ & 6.0 & 7.0 & 4.2 & 1.9 & 5.7 & 5.6 \\
\hline $\begin{array}{l}\text { 13: Infrarenal aortic aneurysm } \\
\text { repair (PDG10-PDG24, PDQ10) }\end{array}$ & 4.8 & 2.5 & 4.2 & 0.6 & 7.2 & 2.9 \\
\hline $\begin{array}{l}\text { 14: Femoropopliteal bypass } \\
\text { (PEH) }\end{array}$ & 6.2 & 11.5 & 16.8 & 4.4 & 3.4 & 3.6 \\
\hline $\begin{array}{l}\text { 15: Stem cell transplantation } \\
\left(\text { not included }^{3}\right)\end{array}$ & 6.2 & 4.2 & 0.0 & 0.0 & 0.0 & 0.0 \\
\hline $\begin{array}{l}\text { 16: Colonoscopy with or with- } \\
\text { out biopsy (JFA15, UJF32, } \\
\text { UJF35, UJF42, UJF45) }\end{array}$ & 264.8 & 61.1 & 36.5 & 115.5 & 213.6 & 130.0 \\
\hline $\begin{array}{l}\text { 17: Colectomy } \\
\text { (JFB20-JFB64, JFH) } \\
\text { Of which: }\end{array}$ & 92.8 & 66.0 & 68.8 & 54.3 & 88.2 & 136.4 \\
\hline $\begin{array}{l}\text { 17A: Laparoscopic colectomy } \\
\text { (JFB21, JFB31, JFB34, JFB41, } \\
\text { JFB44, JFB47, JFB51, JFB61, } \\
\text { JFB64, JFH01, JFH11) }\end{array}$ & 41.0 & 27.5 & 1.4 & 21.9 & 31.2 & 9.9 \\
\hline
\end{tabular}


Table 3.5.2 Eight surgical procedures often carried out as day surgery; total rate and day surgery rate per 100000 inhabitants and day surgery as a percentage of all procedures by gender $2014^{1}$

\begin{tabular}{|c|c|c|c|c|c|c|c|c|}
\hline & \multicolumn{2}{|c|}{ Denmark } & \multicolumn{2}{|c|}{ Finland } & \multicolumn{2}{|c|}{ Norway } & \multicolumn{2}{|c|}{ Sweden } \\
\hline & M & W & M & W & M & W & M & W \\
\hline \multicolumn{9}{|l|}{ Cataract surgery } \\
\hline \multicolumn{9}{|l|}{ (CJC, CJD, CJE, CJF) } \\
\hline Total rate per 100000 inhabitants & 684 & 957 & 590 & 892 & 350 & 491 & 531 & 770 \\
\hline Of which day surgery & 674 & 944 & 570 & 869 & 332 & 473 & 511 & 752 \\
\hline Day surgery $\%$ of total & 99 & 99 & 97 & 97 & 95 & 96 & 96 & 98 \\
\hline \multicolumn{9}{|l|}{ Tonsillectomy (EMB10-20) } \\
\hline Total rate per 100000 inhabitants & 94 & 119 & 132 & 155 & 138 & 174 & 114 & 113 \\
\hline Of which day surgery & 37 & 56 & 99 & 121 & 75 & 107 & 68 & 69 \\
\hline Day surgery $\%$ of total & 39 & 48 & 75 & 78 & 54 & 62 & 59 & 61 \\
\hline \multicolumn{9}{|l|}{$\begin{array}{l}\text { Diagnostic bronchoscopy with or } \\
\text { without biopsy (UGC) }\end{array}$} \\
\hline Total rate per 100000 inhabitants & 303 & 232 & 55 & 34 & 190 & 136 & 113 & 97 \\
\hline Of which day surgery & 213 & 180 & 2 & 1 & 83 & 65 & 73 & 66 \\
\hline Day surgery $\%$ of total & 70 & 77 & 4 & 4 & 44 & 48 & 65 & 68 \\
\hline \multicolumn{9}{|l|}{$\begin{array}{l}\text { Colonoscopy with or without biopsy } \\
\text { (JFA15, UJF32, UJF35, UJF42, UJF45) }\end{array}$} \\
\hline Total rate per 100000 inhabitants & 2677 & 2566 & 69 & 77 & 1268 & 1438 & 913 & 1017 \\
\hline Of which day surgery & 2391 & 2301 & 10 & 16 & 1071 & 1224 & 790 & 887 \\
\hline Day surgery $\%$ of total & 89 & 90 & 14 & 21 & 84 & 85 & 87 & 87 \\
\hline \multicolumn{9}{|l|}{$\begin{array}{l}\text { Laparoscopic cholecystectomy } \\
\text { (JKA 21) }\end{array}$} \\
\hline Total rate per 100000 inhabitants & 82 & 191 & 87 & 184 & 58 & 140 & 80 & 171 \\
\hline Of which day surgery & 40 & 112 & 22 & 69 & 16 & 50 & 19 & 50 \\
\hline Day surgery $\%$ of total & 49 & 59 & 25 & 38 & 28 & 36 & 24 & 29 \\
\hline \multicolumn{9}{|l|}{ Repair of inguinal hernia (JAB) } \\
\hline Total rate per 100000 inhabitants & 352 & 42 & 343 & 39 & 242 & 30 & 237 & 19 \\
\hline Of which day surgery & 288 & 31 & 220 & 22 & 163 & 18 & 178 & 12 \\
\hline Day surgery $\%$ of total & 82 & 74 & 64 & 56 & 67 & 61 & 75 & 64 \\
\hline \multicolumn{9}{|l|}{$\begin{array}{l}\text { Arthroscopic excision of meniscus of } \\
\text { knee (NGD01, NGD11) }\end{array}$} \\
\hline Total rate per 100000 inhabitants & 309 & 193 & 186 & 97 & 312 & 207 & 109 & 63 \\
\hline Of which day surgery & 304 & 188 & 177 & 90 & 295 & 195 & 106 & 60 \\
\hline Day surgery $\%$ of total & 98 & 97 & 95 & 93 & 95 & 94 & 97 & 96 \\
\hline \multicolumn{9}{|l|}{$\begin{array}{l}\text { Excision of mammary gland } \\
\text { (women only) (HAB) }\end{array}$} \\
\hline Total per 100000 inhabitants & 2.7 & 199.3 & 2.5 & 131.2 & 1.9 & 145.2 & 1.9 & 148.8 \\
\hline Of which day surgery & 2.4 & 131.3 & 1.6 & 47.2 & 1.7 & 79.4 & 1.5 & 77.3 \\
\hline Day surgery $\%$ of total & 90 & 66 & 64 & 36 & 93.8 & 54.7 & 76 & 512 \\
\hline
\end{tabular}

1 The NCSP codes refer to the NOMESCO Classification of Surgical Procedures. NCSP-E-version 1.13:2009 NOMESCO 83:2008

Source: The national in-patient registers

Of the surgical procedures shown in Table 3.5.2, cataract surgery shows the highest percentage of day surgery in all the countries (96-98 per cent). The difference in the total rates per inhabitant for cataract surgery is mainly due to lack of reporting in all the countries. There are problems with the definition of day surgery and problems with reporting from private hospitals and clinics. Tonsillectomy is performed as day surgery to various extents and with different totals per capita, which is interest- 
ing in connection with clinical controversy about the indications for this type of surgery and the need for follow-up after the operation. The very low numbers per capita in Finland for bronchoscopy and colonoscopy are because these procedures do not have to be reported nationally. The number of procedures carried out as day surgery varies a great deal from country to country, with higher rates in Denmark for laparoscopic cholecystectomy and with lower rates in Finland for hernia surgery. Norway and Sweden have higher rates for day surgery for partial breast resection.

Table 3.5.3 Proportion of laparoscopic procedures and secondary hip replacements on in-patients by gender, 2014

\begin{tabular}{|c|c|c|c|c|c|c|c|c|c|c|c|c|}
\hline \multirow[b]{2}{*}{ Procedure } & \multicolumn{2}{|c|}{ Denmark } & \multicolumn{2}{|c|}{ Finland } & \multicolumn{2}{|c|}{ Åland ${ }^{1}$} & \multicolumn{2}{|c|}{ Iceland $^{1}$} & \multicolumn{2}{|c|}{ Norway } & \multicolumn{2}{|c|}{ Sweden } \\
\hline & M & W & M & W & M & W & M & W & M & W & M & W \\
\hline \multicolumn{13}{|l|}{ \% laparoscopic } \\
\hline Colectomy & 47 & 44 & 34 & 42 & 4 & 2 & 32 & 40 & 37 & 35 & 8 & 7 \\
\hline Appendectomy & 90 & 83 & 39 & 61 & 5 & 35 & 62 & 74 & 84 & 82 & 50 & 57 \\
\hline Cholecystectomy & 79 & 88 & 77 & 88 & 74 & 87 & 85 & 98 & 88 & 95 & 77 & 87 \\
\hline Repair of inguinal hernia & 40 & 61 & 11 & 17 & 16 & 8 & 23 & 30 & 38 & 33 & 2 & 5 \\
\hline Hysterectomy & .. & 52 & .. & 56 & .. & 6 & .. & 19 & .. & 45 & .. & 23 \\
\hline \multicolumn{13}{|l|}{$\%$ secondary } \\
\hline Hip replacement & 11 & 10 & 15 & 14 & 8 & 10 & 17 & 12 & 12 & 9 & 11 & 8 \\
\hline
\end{tabular}

1 Average 2010-14

Source: The national in-patient registers

The use of laparoscopic methods is shown in Table 3.5.3. Laparoscopic cholecystectomy is very common in all the countries, and almost all cholecystectomies in Iceland are laparoscopic. Furthermore, Table 3.5.3 shows that the numbers for secondary hip replacement are the same for all the countries. It should be noted that the secondary hip replacements that are reported here are not secondary to the primary hip replacements performed in 2014, but mostly secondary to surgery performed many years before.

The detailed Tables 3.5.4-3.5.17 include both surgery on in-patients and surgery carried out as day surgery, which explains the higher rates reported here compared to the per capita numbers shown in Table 3.5.1, which only includes surgery on inpatients. 
Table 3.5.4 Discectomy by age and gender, 2014

\begin{tabular}{|c|c|c|c|c|c|c|c|c|c|c|}
\hline \multirow[b]{2}{*}{ Age } & \multicolumn{2}{|c|}{ Denmark } & \multicolumn{2}{|c|}{ Finland } & \multicolumn{2}{|c|}{ Åland ${ }^{1}$} & \multicolumn{2}{|c|}{ Norway } & \multicolumn{2}{|c|}{ Sweden } \\
\hline & M & W & $M$ & W & $M$ & W & $M$ & W & $M$ & W \\
\hline \multicolumn{11}{|c|}{$\begin{array}{l}\text { Total } \\
\text { number of } \\
\text { procedures }\end{array}$} \\
\hline$<15$ & 1 & 5 & 5 & 5 & - & - & 2 & 5 & - & 12 \\
\hline $15-24$ & 60 & 47 & 79 & 82 & - & 1 & 86 & 82 & 76 & 81 \\
\hline $25-44$ & 1090 & 860 & 1020 & 716 & 3 & 3 & 964 & 866 & 679 & 722 \\
\hline $45-64$ & 2188 & 1813 & 1666 & 1405 & 3 & 3 & 1782 & 1582 & 1309 & 1248 \\
\hline $65-74$ & 1048 & 1026 & 787 & 793 & 3 & 4 & 758 & 700 & 853 & 897 \\
\hline $75-84$ & 513 & 628 & 390 & 644 & 2 & 2 & 341 & 493 & 517 & 620 \\
\hline $85+$ & 42 & 86 & 58 & 69 & - & 1 & 36 & 63 & 63 & 76 \\
\hline Total & 4942 & 4465 & 4005 & 3714 & 11 & 14 & 3969 & 3791 & 3532 & 3656 \\
\hline \multicolumn{11}{|c|}{$\begin{array}{l}\text { Per } 100000 \\
\text { in the age } \\
\text { group }\end{array}$} \\
\hline$<15$ & - & 1 & 1 & 1 & - & - & - & 1 & - & 2 \\
\hline $15-24$ & 16 & 13 & 24 & 26 & - & 39 & 25 & 25 & 12 & 14 \\
\hline $25-44$ & 153 & 123 & 147 & 109 & 78 & 93 & 133 & 126 & 53 & 59 \\
\hline $45-64$ & 291 & 242 & 225 & 188 & 76 & 77 & 268 & 248 & 107 & 103 \\
\hline $65-74$ & 349 & 323 & 276 & 249 & 193 & 270 & 331 & 295 & 159 & 163 \\
\hline $75-84$ & 383 & 370 & 282 & 321 & 272 & 241 & 322 & 360 & 195 & 191 \\
\hline $85+$ & 110 & 109 & 171 & 246 & 119 & 137 & 204 & 155 & 95 & 85 \\
\hline Total & 176 & 157 & 149 & 134 & 76 & 97 & 154 & 148 & 73 & 75 \\
\hline
\end{tabular}

1 Average 2010-14

NCSP: $A B C$

Source: The national in-patient registers

Table 3.5.5 Thyroidectomy by age and gender, 2014

\begin{tabular}{|c|c|c|c|c|c|c|c|c|c|c|}
\hline \multirow[b]{2}{*}{ Age } & \multicolumn{2}{|c|}{ Denmark } & \multicolumn{2}{|c|}{ Finland } & \multicolumn{2}{|c|}{ Åland ${ }^{1}$} & \multicolumn{2}{|c|}{ Norway } & \multicolumn{2}{|c|}{ Sweden } \\
\hline & $M$ & W & $M$ & W & $M$ & W & $M$ & W & $M$ & W \\
\hline \multicolumn{11}{|c|}{$\begin{array}{l}\text { Total } \\
\text { number of } \\
\text { procedures }\end{array}$} \\
\hline$<15$ & 1 & 4 & 4 & 5 & - & - & 4 & 3 & 7 & 14 \\
\hline $15-24$ & 8 & 53 & 10 & 62 & - & - & 15 & 41 & 14 & 127 \\
\hline $25-44$ & 110 & 528 & 81 & 510 & - & 3 & 80 & 398 & 142 & 791 \\
\hline $45-54$ & 134 & 531 & 50 & 393 & - & 2 & 78 & 299 & 120 & 542 \\
\hline $55-64$ & 123 & 416 & 112 & 450 & - & 2 & 83 & 243 & 117 & 370 \\
\hline $65-74$ & 144 & 328 & 98 & 387 & 1 & 1 & 70 & 193 & 131 & 384 \\
\hline $75-84$ & 34 & 105 & 36 & 156 & - & - & 28 & 58 & 59 & 191 \\
\hline $85+$ & 5 & 22 & 6 & 27 & - & - & 7 & 16 & 7 & 25 \\
\hline Total & 559 & 1987 & 397 & 1990 & 1 & 9 & 365 & 1251 & 612 & 2444 \\
\hline \multicolumn{11}{|c|}{$\begin{array}{l}\text { Per } 100000 \\
\text { in the age } \\
\text { group }\end{array}$} \\
\hline$<15$ & - & 1 & 1 & 1 & - & - & 1 & 1 & 1 & 2 \\
\hline $15-24$ & 2 & 15 & 3 & 19 & - & 13 & 4 & 13 & 2 & 22 \\
\hline $25-44$ & 15 & 75 & 12 & 78 & 6 & 87 & 11 & 58 & 11 & 64 \\
\hline $45-54$ & 33 & 133 & 13 & 107 & 20 & 117 & 22 & 88 & 18 & 85 \\
\hline $55-64$ & 36 & 119 & 30 & 118 & 10 & 105 & 27 & 82 & 20 & 65 \\
\hline $65-74$ & 48 & 103 & 34 & 121 & 39 & 95 & 31 & 81 & 24 & 70 \\
\hline 75-84 & 25 & 62 & 26 & 78 & - & - & 26 & 42 & 22 & 59 \\
\hline $85+$ & 13 & 28 & 16 & 29 & - & 38 & 19 & 21 & 7 & 14 \\
\hline Total & 20 & 70 & 15 & 72 & 10 & 66 & 14 & 49 & 13 & 50 \\
\hline
\end{tabular}

1 Average 2010-14

NCSP: BAA 20-60

Source: The national in-patient registers 
Table 3.5.6 Cataract surgery by age and gender, 2014

\begin{tabular}{|c|c|c|c|c|c|c|c|c|c|c|}
\hline \multirow[b]{2}{*}{ Age } & \multicolumn{2}{|c|}{ Denmark } & \multicolumn{2}{|c|}{ Finland } & \multicolumn{2}{|c|}{ Åland ${ }^{1}$} & \multicolumn{2}{|c|}{ Norway } & \multicolumn{2}{|c|}{ Sweden } \\
\hline & M & W & $M$ & W & $M$ & W & $M$ & W & $M$ & W \\
\hline \multicolumn{11}{|c|}{$\begin{array}{l}\text { Total } \\
\text { number of } \\
\text { procedures }\end{array}$} \\
\hline$<45$ & 296 & 273 & 242 & 190 & - & - & 215 & 145 & 429 & 386 \\
\hline $45-64$ & 3211 & 4072 & 2914 & 3081 & 2 & 1 & 1406 & 1537 & 3990 & 4491 \\
\hline $65-74$ & 6802 & 10075 & 5328 & 8069 & 1 & 1 & 2759 & 3852 & 8642 & 12660 \\
\hline $75-84$ & 6982 & 9946 & 5888 & 10641 & 2 & 2 & 3452 & 5050 & 9538 & 14936 \\
\hline $85+$ & 1883 & 2822 & 1488 & 2766 & 1 & 2 & 1199 & 1960 & 3123 & 4916 \\
\hline Total & 19174 & 27188 & 15860 & 24747 & 6 & 6 & 9031 & 12544 & 25722 & 37389 \\
\hline \multicolumn{11}{|c|}{$\begin{array}{l}\text { Per } 100000 \\
\text { in the age } \\
\text { group }\end{array}$} \\
\hline$<45$ & 19 & 18 & 16 & 13 & - & - & 14 & 10 & 16 & 15 \\
\hline $45-64$ & 427 & 544 & 393 & 412 & 46 & 19 & 211 & 241 & 325 & 372 \\
\hline $65-74$ & 2264 & 3168 & 1869 & 2531 & 64 & 41 & 1205 & 1625 & 1616 & 2300 \\
\hline $75-84$ & 5210 & 5859 & 4260 & 5311 & 326 & 263 & 3261 & 3688 & 3605 & 4593 \\
\hline $85+$ & 4954 & 3587 & 3957 & 2944 & 255 & 381 & 3219 & 2532 & 3268 & 2713 \\
\hline Total & 684 & 957 & 590 & 892 & 41 & 41 & 350 & 491 & 531 & 770 \\
\hline
\end{tabular}

1 Average 2010-14

NCSP: CJC, CJD, CJE, CJF

Source: The national in-patient registers

Table 3.5.7.a Transluminal coronary angioplasty (PTCA, PCI) by age, men 2014

\begin{tabular}{|c|c|c|c|c|}
\hline Age & Denmark & Finland & Norway & Sweden \\
\hline \multicolumn{5}{|c|}{$\begin{array}{l}\text { Total number of } \\
\text { procedures }\end{array}$} \\
\hline$<45$ & 321 & 227 & 340 & 347 \\
\hline $45-54$ & 1268 & 928 & 1341 & 1708 \\
\hline $55-64$ & 1923 & 2007 & 2722 & 3650 \\
\hline $65-74$ & 2313 & 2348 & 2889 & 5322 \\
\hline $75-84$ & 1272 & 1631 & 1623 & 3115 \\
\hline $85+$ & 224 & 314 & 310 & 561 \\
\hline Total & 7321 & 7455 & 9225 & 14703 \\
\hline \multicolumn{5}{|c|}{ Per 100000 in the } \\
\hline \multicolumn{5}{|c|}{ age group } \\
\hline$<45$ & 20 & 15 & 22 & 13 \\
\hline $45-54$ & 311 & 249 & 370 & 260 \\
\hline $55-64$ & 559 & 543 & 897 & 637 \\
\hline $65-74$ & 770 & 824 & 1262 & 995 \\
\hline $75-84$ & 949 & 1180 & 1533 & 1177 \\
\hline $85+$ & 589 & 835 & 832 & 587 \\
\hline Total & 261 & 278 & 357 & 304 \\
\hline
\end{tabular}

NCSP: FNG 02; FNG 05

Source: The national in-patient registers 
Morbidity, Medical Treatment, Accidents and Pharmaceutical products

Table 3.5.7.b Transluminal coronary angioplasty (PTCA, PCI) by age, women 2014

\begin{tabular}{|c|c|c|c|c|}
\hline Age & Denmark & Finland & Norway & Sweden \\
\hline \multicolumn{5}{|c|}{$\begin{array}{l}\text { Total number of } \\
\text { procedures }\end{array}$} \\
\hline$<45$ & 78 & 38 & 62 & 73 \\
\hline $45-54$ & 284 & 166 & 268 & 394 \\
\hline $55-64$ & 523 & 489 & 586 & 933 \\
\hline $65-74$ & 762 & 916 & 967 & 1709 \\
\hline $75-84$ & 690 & 1043 & 761 & 1611 \\
\hline $85+$ & 188 & 314 & 201 & 545 \\
\hline Total & 2525 & 2966 & 2845 & 5265 \\
\hline \multicolumn{5}{|c|}{ Per 100000 in the } \\
\hline \multicolumn{5}{|c|}{ age group } \\
\hline$<45$ & 5 & 3 & 4 & 3 \\
\hline $45-54$ & 71 & 45 & 78 & 62 \\
\hline $55-64$ & 150 & 128 & 198 & 164 \\
\hline $65-74$ & 240 & 287 & 408 & 310 \\
\hline $75-84$ & 406 & 521 & 556 & 495 \\
\hline $85+$ & 239 & 334 & 260 & 301 \\
\hline Total & 89 & 107 & 111 & 108 \\
\hline
\end{tabular}

NCSP: FNG 02; FNG 05

Source: The national in-patient registers

Table 3.5.7 shows that the highest rates for PTCA are found for both men and women in the age group 75-84 years for all countries. 
Table 3.5.8.a Coronary artery bypass graft by age, men 2014

\begin{tabular}{|c|c|c|c|c|}
\hline Age & Denmark & Finland & Norway & Sweden \\
\hline \multicolumn{5}{|c|}{$\begin{array}{l}\text { Total number of } \\
\text { procedures }\end{array}$} \\
\hline$<45$ & 26 & 11 & 19 & 19 \\
\hline $45-54$ & 148 & 86 & 184 & 168 \\
\hline $55-64$ & 418 & 336 & 434 & 564 \\
\hline $65-74$ & 720 & 476 & 623 & 1044 \\
\hline $75-84$ & 324 & 296 & 317 & 561 \\
\hline $85+$ & 13 & 12 & 27 & 29 \\
\hline Total & 1649 & 1217 & 1604 & 2385 \\
\hline \multicolumn{5}{|c|}{ Per 100000 in the } \\
\hline \multicolumn{5}{|c|}{ age group } \\
\hline$<45$ & 2 & 1 & 1 & 7 \\
\hline $45-54$ & 36 & 23 & 51 & 26 \\
\hline $55-64$ & 121 & 91 & 143 & 98 \\
\hline $65-74$ & 240 & 167 & 272 & 195 \\
\hline $75-84$ & 242 & 214 & 299 & 212 \\
\hline $85+$ & 34 & 32 & 72 & 30 \\
\hline Total & 59 & 45 & 62 & 49 \\
\hline
\end{tabular}

NCSP: FNC, FND, FNE

Source: The national in-patient registers

Table 3.5.8.b Coronary artery bypass graft by age, women 2014

\begin{tabular}{|c|c|c|c|c|}
\hline Age & Denmark & Finland & Norway & Sweden \\
\hline \multicolumn{5}{|c|}{$\begin{array}{l}\text { Total number of } \\
\text { procedures }\end{array}$} \\
\hline$<45$ & 5 & 4 & 4 & 4 \\
\hline $45-54$ & 23 & 12 & 21 & 42 \\
\hline $55-64$ & 73 & 53 & 61 & 96 \\
\hline $65-74$ & 139 & 114 & 168 & 209 \\
\hline $75-84$ & 126 & 140 & 115 & 202 \\
\hline $85+$ & 2 & 11 & 10 & 12 \\
\hline Total & 368 & 334 & 379 & 565 \\
\hline \multicolumn{5}{|c|}{ Per 100000 in the } \\
\hline \multicolumn{5}{|c|}{ age group } \\
\hline$<45$ & 0 & - & 0 & 0 \\
\hline $45-54$ & 6 & - & 6 & 7 \\
\hline $55-64$ & 21 & 14 & 21 & 17 \\
\hline $65-74$ & 44 & 36 & 71 & 38 \\
\hline $75-84$ & 74 & 70 & 84 & 62 \\
\hline $85+$ & 3 & 12 & 13 & 7 \\
\hline Total & 13 & 12 & 15 & 12 \\
\hline
\end{tabular}

NCSP: FNC, FND, FNE

Source: The national in-patient registers 
Morbidity, Medical Treatment, Accidents and Pharmaceutical products

Table 3.5.9.a Appendectomy by age, men 2014

\begin{tabular}{|c|c|c|c|c|c|}
\hline Age & Denmark & Finland & Åland ${ }^{1}$ & Norway & Sweden \\
\hline \multicolumn{6}{|c|}{$\begin{array}{l}\text { Total number } \\
\text { of procedures }\end{array}$} \\
\hline$<15$ & 635 & 390 & 4 & 444 & 1110 \\
\hline $15-24$ & 790 & 626 & 5 & 817 & 752 \\
\hline $25-44$ & 944 & 1190 & 7 & 1008 & 2030 \\
\hline $45-64$ & 612 & 789 & 4 & 616 & 1162 \\
\hline $65+$ & 375 & 386 & 3 & 290 & 641 \\
\hline Total & 3356 & 3381 & 23 & 3292 & 6335 \\
\hline \multicolumn{6}{|c|}{$\begin{array}{l}\text { Per } 100000 \text { in } \\
\text { the age group }\end{array}$} \\
\hline$<15$ & 128 & 85 & 165 & 93 & 134 \\
\hline $15-24$ & 212 & 188 & 321 & 237 & 123 \\
\hline $25-44$ & 133 & 172 & 184 & 139 & 159 \\
\hline $45-64$ & 81 & 106 & 106 & 93 & 95 \\
\hline $65+$ & 79 & 84 & 111 & 78 & 72 \\
\hline Total & 120 & 126 & 162 & 127 & 131 \\
\hline
\end{tabular}

1 Average 2010-14

NCSP: JEA

Source: The national in-patient registers

Table 3.5.9.b Appendectomy by age, women 2014

\begin{tabular}{|c|c|c|c|c|c|}
\hline Age & Denmark & Finland & Åland ${ }^{1}$ & Norway & Sweden \\
\hline \multicolumn{6}{|c|}{$\begin{array}{l}\text { Total number of } \\
\text { procedures }\end{array}$} \\
\hline$<15$ & 452 & 285 & 3 & 355 & 780 \\
\hline $15-24$ & 807 & 779 & 4 & 845 & 1362 \\
\hline $25-44$ & 994 & 1121 & 6 & 1002 & 1933 \\
\hline $45-64$ & 828 & 977 & 2 & 646 & 1452 \\
\hline $65+$ & 537 & 463 & 2 & 424 & 923 \\
\hline Total & 3618 & 3625 & 17 & 3272 & 6450 \\
\hline \multicolumn{6}{|c|}{$\begin{array}{l}\text { Per } 100000 \text { in } \\
\text { the age group }\end{array}$} \\
\hline$<15$ & 96 & 65 & 144 & 78 & 99 \\
\hline $15-24$ & 227 & 244 & 262 & 260 & 236 \\
\hline $25-44$ & 142 & 170 & 163 & 146 & 157 \\
\hline $45-64$ & 111 & 131 & 53 & 101 & 120 \\
\hline $65+$ & 95 & 76 & 69 & 94 & 87 \\
\hline Total & 127 & 131 & 119 & 128 & 133 \\
\hline
\end{tabular}

1 Average 2010-14

NCSP: JEA

Source: The national in-patient registers 
Table 3.5.10.a Cholecystectomy by age, men 2014

\begin{tabular}{|c|c|c|c|c|c|}
\hline Age & Denmark & Finland & Åland ${ }^{1}$ & Norway & Sweden \\
\hline \multicolumn{6}{|c|}{$\begin{array}{l}\text { Total number } \\
\text { of procedures }\end{array}$} \\
\hline$<25$ & 92 & 45 & 0 & 46 & 139 \\
\hline $25-44$ & 647 & 478 & 2 & 398 & 1132 \\
\hline $45-64$ & 1128 & 1136 & 6 & 710 & 1952 \\
\hline $65+$ & 767 & 1214 & 6 & 495 & 1586 \\
\hline Total & 2634 & 2873 & 15 & 1649 & 4809 \\
\hline \multicolumn{6}{|c|}{$\begin{array}{l}\text { Per } 100000 \text { in } \\
\text { the age group }\end{array}$} \\
\hline$<25$ & 11 & 6 & 5 & 6 & 10 \\
\hline $25-44$ & 91 & 69 & 61 & 55 & 89 \\
\hline $45-64$ & 150 & 153 & 147 & 107 & 159 \\
\hline $65+$ & 162 & 263 & 253 & 133 & 177 \\
\hline Total & 94 & 107 & 103 & 64 & 99 \\
\hline
\end{tabular}

1 Average 2010-14

NCSP: JKA 20-21

Source: The national in-patient registers

Table 3.5.10.b Cholecystectomy by age, women 2014

\begin{tabular}{|c|c|c|c|c|c|}
\hline Age & Denmark & Finland & Åland ${ }^{1}$ & Norway & Sweden \\
\hline \multicolumn{6}{|c|}{$\begin{array}{l}\text { Total number } \\
\text { of procedures }\end{array}$} \\
\hline$<25$ & 410 & 232 & 1 & 254 & 636 \\
\hline $25-44$ & 2022 & 1484 & 9 & 1363 & 3175 \\
\hline $45-64$ & 2172 & 2312 & 12 & 1366 & 3399 \\
\hline $65+$ & 1145 & 1515 & 7 & 726 & 1963 \\
\hline Total & 5749 & 5543 & 29 & 3709 & 9173 \\
\hline \multicolumn{6}{|c|}{ Per 100000 in } \\
\hline$<25$ & 50 & 31 & 32 & 33 & 47 \\
\hline $25-44$ & 289 & 226 & 251 & 94 & 259 \\
\hline $45-64$ & 290 & 309 & 299 & 214 & 282 \\
\hline $65+$ & 202 & 247 & 226 & 161 & 186 \\
\hline Total & 202 & 200 & 202 & 145 & 189 \\
\hline
\end{tabular}

1 Average 2010-14

NCSP: JKA 20-21

Source: The national in-patient registers 
Morbidity, Medical Treatment, Accidents and Pharmaceutical products

Table 3.5.11 Transplantation of the kidney by age and gender, 2014

\begin{tabular}{|c|c|c|c|c|c|c|c|c|c|c|}
\hline \multirow[b]{2}{*}{ Age } & \multicolumn{2}{|c|}{ Denmark } & \multicolumn{2}{|c|}{ Finland } & \multicolumn{2}{|c|}{ Åland ${ }^{1}$} & \multicolumn{2}{|c|}{ Norway } & \multicolumn{2}{|c|}{ Sweden } \\
\hline & M & W & $M$ & W & $M$ & W & $M$ & W & $M$ & W \\
\hline \multicolumn{11}{|c|}{$\begin{array}{l}\text { Total } \\
\text { number of } \\
\text { procedures }\end{array}$} \\
\hline$<15$ & 5 & 2 & 7 & 5 & - & - & 4 & 3 & 12 & 1 \\
\hline $15-24$ & 9 & 2 & 5 & 3 & - & - & 6 & 3 & 13 & 7 \\
\hline $25-44$ & 44 & 20 & 31 & 16 & - & - & 40 & 17 & 58 & 55 \\
\hline $45-54$ & 40 & 29 & 33 & 25 & - & - & 41 & 23 & 89 & 36 \\
\hline $55-64$ & 38 & 20 & 37 & 22 & - & - & 51 & 17 & 57 & 42 \\
\hline $65+$ & 26 & 16 & 35 & 18 & 1 & - & 53 & 23 & 58 & 37 \\
\hline Total & 162 & 89 & 148 & 89 & 1 & 1 & 195 & 86 & 287 & 178 \\
\hline \multicolumn{11}{|c|}{$\begin{array}{l}\text { Per } 100000 \\
\text { in the age } \\
\text { group }\end{array}$} \\
\hline $\begin{array}{l}\text { group } \\
<15\end{array}$ & 1 & - & 2 & 1 & - & - & 1 & 1 & 1 & - \\
\hline $15-24$ & 2 & 1 & 2 & 1 & - & - & 2 & 1 & 2 & 1 \\
\hline $25-44$ & 6 & 3 & 4 & 2 & - & - & 6 & 2 & 5 & 4 \\
\hline $45-54$ & 10 & 7 & 9 & 7 & 20 & - & 11 & 7 & 14 & 6 \\
\hline $55-64$ & 11 & 6 & 10 & 6 & 10 & 10 & 17 & 6 & 10 & 7 \\
\hline $65+$ & 6 & 3 & 8 & 3 & 32 & 14 & 14 & 5 & 6 & 4 \\
\hline Total & 6 & 3 & 6 & 3 & 10 & 4 & 8 & 3 & 6 & 4 \\
\hline
\end{tabular}

1 Average 2010-14

NCSP: KASO0-KAS20

Source: The national in-patient registers

As shown in Table 3.5.11, kidney transplants are performed in almost all of the countries more often on men than women. Apparently, this also applies to all age groups. Whether this reflects differences in morbidity between men and women or whether it is a possible effect of gender discrimination should be addressed. 
Table 3.5.12 Open prostatectomy by age, men 2014

\begin{tabular}{|c|c|c|c|c|c|}
\hline Age & Denmark & Finland & Åland ${ }^{1}$ & Norway & Sweden \\
\hline \multicolumn{6}{|c|}{$\begin{array}{l}\text { Total number } \\
\text { of procedures }\end{array}$} \\
\hline$<45$ & 4 & 10 & - & 4 & 8 \\
\hline $45-64$ & 525 & 625 & 6 & 757 & 1316 \\
\hline $65-74$ & 597 & 523 & 4 & 939 & 1820 \\
\hline $75-84$ & 29 & 28 & - & 72 & 141 \\
\hline $85+$ & 4 & 2 & - & 5 & 6 \\
\hline Total & 1159 & 1188 & 10 & 1777 & 3292 \\
\hline \multicolumn{6}{|c|}{$\begin{array}{l}\text { Per } 100000 \text { in } \\
\text { the age group }\end{array}$} \\
\hline$<45$ & - & 1 & - & - & 0,3 \\
\hline $45-64$ & 70 & 84 & 152 & 114 & 107 \\
\hline $65-74$ & 199 & 183 & 244 & 410 & 340 \\
\hline $75-84$ & 22 & 20 & - & 68 & 53 \\
\hline $85+$ & 11 & 5 & - & 13 & 6 \\
\hline Total & 41 & 44 & 69 & 69 & 68 \\
\hline
\end{tabular}

1 Average 2010-14

NCSP: KEC; KED00; KED96

Source: The national in-patient registers

Table 3.5.12 shows that open prostatectomy is most common in the age group 6574.

Table 3.5.13 Transurethral prostatectomy by age, men 2014

\begin{tabular}{|c|c|c|c|c|c|}
\hline Age & Denmark & Finland & Åland ${ }^{1}$ & Norway & Sweden \\
\hline \multicolumn{6}{|c|}{$\begin{array}{l}\text { Total number } \\
\text { of procedures }\end{array}$} \\
\hline$<45$ & 20 & 4 & - & 11 & 8 \\
\hline $45-64$ & 716 & 687 & 5 & 634 & 848 \\
\hline $65-74$ & 1398 & 1564 & 9 & 1408 & 2247 \\
\hline $75-84$ & 813 & 1156 & 7 & 1030 & 1707 \\
\hline $85+$ & 140 & 277 & 2 & 348 & 427 \\
\hline Total & 3087 & 3688 & 23 & 3431 & 5237 \\
\hline \multicolumn{6}{|c|}{$\begin{array}{l}\text { Per } 100000 \text { in } \\
\text { the age group }\end{array}$} \\
\hline$<45$ & 1 & - & - & 1 & - \\
\hline $45-64$ & 95 & 93 & 132 & 95 & 69 \\
\hline $65-74$ & 465 & 549 & 565 & 615 & 420 \\
\hline $75-84$ & 607 & 836 & 979 & 973 & 645 \\
\hline $85+$ & 368 & 737 & 765 & 934 & 447 \\
\hline Total & 110 & 137 & 162 & 133 & 108 \\
\hline
\end{tabular}

1 Average 2010-14

NCSP: KED22; KED52-KED72; KED98

Source: The national in-patient registers 
Table 3.5.14 Hysterectomy by age, women 2014

\begin{tabular}{|c|c|c|c|c|c|}
\hline Age & Denmark & Finland & Åland ${ }^{1}$ & Norway & Sweden \\
\hline \multicolumn{6}{|c|}{$\begin{array}{l}\text { Total number } \\
\text { of procedures }\end{array}$} \\
\hline$<25$ & 12 & 6 & - & 22 & 21 \\
\hline $25-44$ & 1646 & 1171 & 12 & 1340 & 1891 \\
\hline $45-64$ & 3277 & 2882 & 29 & 2451 & 4073 \\
\hline $65+$ & 1366 & 1146 & 10 & 964 & 2239 \\
\hline Total & 6301 & 5205 & 51 & 4777 & 8224 \\
\hline \multicolumn{6}{|c|}{$\begin{array}{l}\text { Per } 100000 \text { in } \\
\text { the age group }\end{array}$} \\
\hline$<25$ & 1 & 1 & 5 & 3 & 2 \\
\hline $25-44$ & 235 & 178 & 338 & 196 & 154 \\
\hline $45-64$ & 438 & 385 & 694 & 384 & 338 \\
\hline $65+$ & 241 & 187 & 350 & 214 & 212 \\
\hline Total & 222 & 188 & 356 & 187 & 169 \\
\hline
\end{tabular}

1 Average 2010-14

NCSP: LCC, LCD

Source: The national in-patient registers

Table 3.5.15 Caesarean section by age, women 2014

\begin{tabular}{|c|c|c|c|c|c|}
\hline Age & Denmark & Finland & Åland ${ }^{1}$ & Norway & Sweden \\
\hline \multicolumn{6}{|c|}{$\begin{array}{l}\text { Total number } \\
\text { of procedures }\end{array}$} \\
\hline$<15$ & 1 & - & - & - & 2 \\
\hline $15-24$ & 1152 & 1100 & 37 & 895 & 1830 \\
\hline $25-34$ & 7587 & 5382 & 210 & 5844 & 11440 \\
\hline $35-44$ & 3428 & 2496 & 102 & 3007 & 5945 \\
\hline $45+$ & 49 & 50 & 2 & 71 & 115 \\
\hline Total & 12217 & 9028 & 351 & 9817 & 19332 \\
\hline \multicolumn{6}{|c|}{$\begin{array}{l}\text { Per } 1000 \\
\text { deliveries }^{2}\end{array}$} \\
\hline$<15$ & - & - & - & - & 400 \\
\hline $15-24$ & 167 & 118 & 216 & 108 & 115 \\
\hline $25-34$ & 202 & 149 & 231 & 150 & 154 \\
\hline $35-44$ & 278 & 216 & 340 & 257 & 241 \\
\hline $45+$ & 462 & 345 & 1000 & 559 & 402 \\
\hline Total & 215 & 158 & 254 & 166 & 168 \\
\hline
\end{tabular}

1 Average 2010-14

2 Sweden and Norway NCSP: MCA

Source: The national in-patient registers 
Table 3.5.16.a Hip replacement by age, men 2014

\begin{tabular}{lrrrrr}
\hline Age & Denmark & Finland & Åland $^{1}$ & Norway & Sweden \\
\hline $\begin{array}{l}\text { Total number of } \\
\text { procedures }\end{array}$ & & & & & \\
$<25$ & 23 & 11 & - & 16 & 17 \\
$25-44$ & 173 & 159 & 1 & 140 & 171 \\
$45-64$ & 1426 & 1651 & 8 & 1106 & 2063 \\
$65-74$ & 1876 & 1776 & 9 & 1349 & 2724 \\
$75+$ & 1813 & 1836 & 8 & 1751 & 3100 \\
Total & 511 & 5433 & 26 & 4362 & 8075 \\
Per 100 000 in the & & & & & \\
age group & 3 & 1 & & & 13 \\
$<25$ & 24 & 23 & 17 & 19 & 168 \\
$25-44$ & 190 & 223 & 203 & 166 & 509 \\
$45-64$ & 624 & 623 & 590 & 589 & 167 \\
$65-74$ & 1054 & 1044 & 865 & 1224 & 169 \\
$75+$ & 190 & 202 & 185 & & \\
Total & & & & & \\
\hline
\end{tabular}

1 Average 2010-14

NCSP: NFB, NFC

Source: The national in-patient registers

Table 3.5.16.b Hip replacement by age, women 2014

\begin{tabular}{|c|c|c|c|c|c|}
\hline Age & Denmark & Finland & Åland ${ }^{1}$ & Norway & Sweden \\
\hline \multicolumn{6}{|c|}{$\begin{array}{l}\text { Total number of } \\
\text { procedures }\end{array}$} \\
\hline$<25$ & 21 & 8 & - & 16 & 13 \\
\hline $25-44$ & 112 & 115 & - & 124 & 167 \\
\hline $45-64$ & 1575 & 1723 & 7 & 1610 & 2109 \\
\hline $65-74$ & 2520 & 2383 & 12 & 2570 & 3616 \\
\hline $75+$ & 3669 & 3698 & 15 & 4370 & 5804 \\
\hline Total & 7897 & 7927 & 34 & 8690 & 11709 \\
\hline \multicolumn{6}{|c|}{ Per 100000 in the } \\
\hline \multicolumn{6}{|c|}{ age group } \\
\hline$<25$ & 3 & 1 & - & 2 & 1 \\
\hline $25-44$ & 16 & 17 & 12 & 18 & 14 \\
\hline $45-64$ & 211 & 230 & 173 & 252 & 175 \\
\hline $65-74$ & 792 & 747 & 783 & 1084 & 657 \\
\hline $75+$ & 1477 & 1256 & 1030 & 2039 & 1146 \\
\hline Total & 278 & 286 & 239 & 340 & 241 \\
\hline
\end{tabular}

1 Average 2010-14

NCSP: NFB, NFC

Source: The national in-patient registers

Table 3.5.16 shows that Norway not only has the highest total rate for hip replacement for women, but also has the highest number in all the age groups above 45 years. 
Table 3.5.17.a Total knee replacement by age, men 2014

\begin{tabular}{|c|c|c|c|c|c|}
\hline Age & Denmark & Finland & Åland ${ }^{1}$ & Norway & Sweden \\
\hline \multicolumn{6}{|c|}{$\begin{array}{l}\text { Total number of } \\
\text { procedures }\end{array}$} \\
\hline$<25$ & 3 & - & - & - & 2 \\
\hline $25-44$ & 41 & 32 & - & 21 & 28 \\
\hline $45-64$ & 1023 & 1201 & 6 & 646 & 1291 \\
\hline $65-74$ & 1300 & 1324 & 9 & 830 & 1831 \\
\hline $75+$ & 716 & 858 & 4 & 486 & 1180 \\
\hline Total & 3083 & 3415 & 20 & 1983 & 4332 \\
\hline \multicolumn{6}{|c|}{ Per 100000 in the } \\
\hline \multicolumn{6}{|c|}{ age group } \\
\hline$<25$ & - & - & - & - & 0 \\
\hline $25-44$ & 6 & 5 & - & 3 & 2 \\
\hline $45-64$ & 136 & 162 & 157 & 97 & 105 \\
\hline $65-74$ & 433 & 465 & 578 & 362 & 342 \\
\hline $75+$ & 416 & 488 & 453 & 340 & 328 \\
\hline Total & 110 & 127 & 138 & 77 & 89 \\
\hline
\end{tabular}

1 Average 2010-14

NCSP: NGB20, NGB30, NGB40

Source: The national in-patient registers

Table 3.5.17.b Total knee replacement by age, women 2014

\begin{tabular}{|c|c|c|c|c|c|}
\hline Age & Denmark & Finland & Åland ${ }^{1}$ & Norway & Sweden \\
\hline \multicolumn{6}{|c|}{$\begin{array}{l}\text { Total number of } \\
\text { procedures }\end{array}$} \\
\hline$<25$ & 2 & - & - & 3 & 2 \\
\hline $25-44$ & 53 & 31 & - & 45 & 38 \\
\hline $45-64$ & 1383 & 1894 & 10 & 922 & 1753 \\
\hline $65-74$ & 1768 & 2383 & 10 & 1233 & 2375 \\
\hline $75+$ & 1241 & 1948 & 8 & 909 & 1889 \\
\hline Total & 4447 & 6256 & 27 & 3112 & 6057 \\
\hline \multicolumn{6}{|c|}{ Per 100000 in the } \\
\hline \multicolumn{6}{|c|}{ age group } \\
\hline$<25$ & - & - & - & 0 & 0 \\
\hline $25-44$ & 8 & 5 & - & 7 & 3 \\
\hline $45-64$ & 185 & 253 & 236 & 144 & 145 \\
\hline $65-74$ & 556 & 747 & 648 & 520 & 431 \\
\hline $75+$ & 500 & 662 & 557 & 424 & 373 \\
\hline Total & 156 & 225 & 192 & 122 & 125 \\
\hline
\end{tabular}

1 Average 2010-14

NCSP: NGB20, NGB30, NGB40

Source: The national in-patient registers 
Table 3.5.17 shows that Finland has the highest total rate for knee replacements and the highest rate for this procedure in all age groups above 45 years.

Figure 3.5.1 shows increased rates for percutaneous transluminal coronary angioplasty (PTCA) and slightly decreased rates for coronary anastomosis operations for the period 2003-2014. In general, the countries maintain their relative position over time. The HDP2 list defines coronary anastomosis operations a little less widely than in NOMESCO's earlier statistical data, but this does not explain the lower rates for 2008 and 2009. 
Morbidity, Medical Treatment, Accidents and Pharmaceutical products

Table 3.5.1 Transluminal coronary angioplasty and coronary anastomosis surgery, total rates per 100000 inhabitants, 2003-2014

Transluminal coronary angioplasty
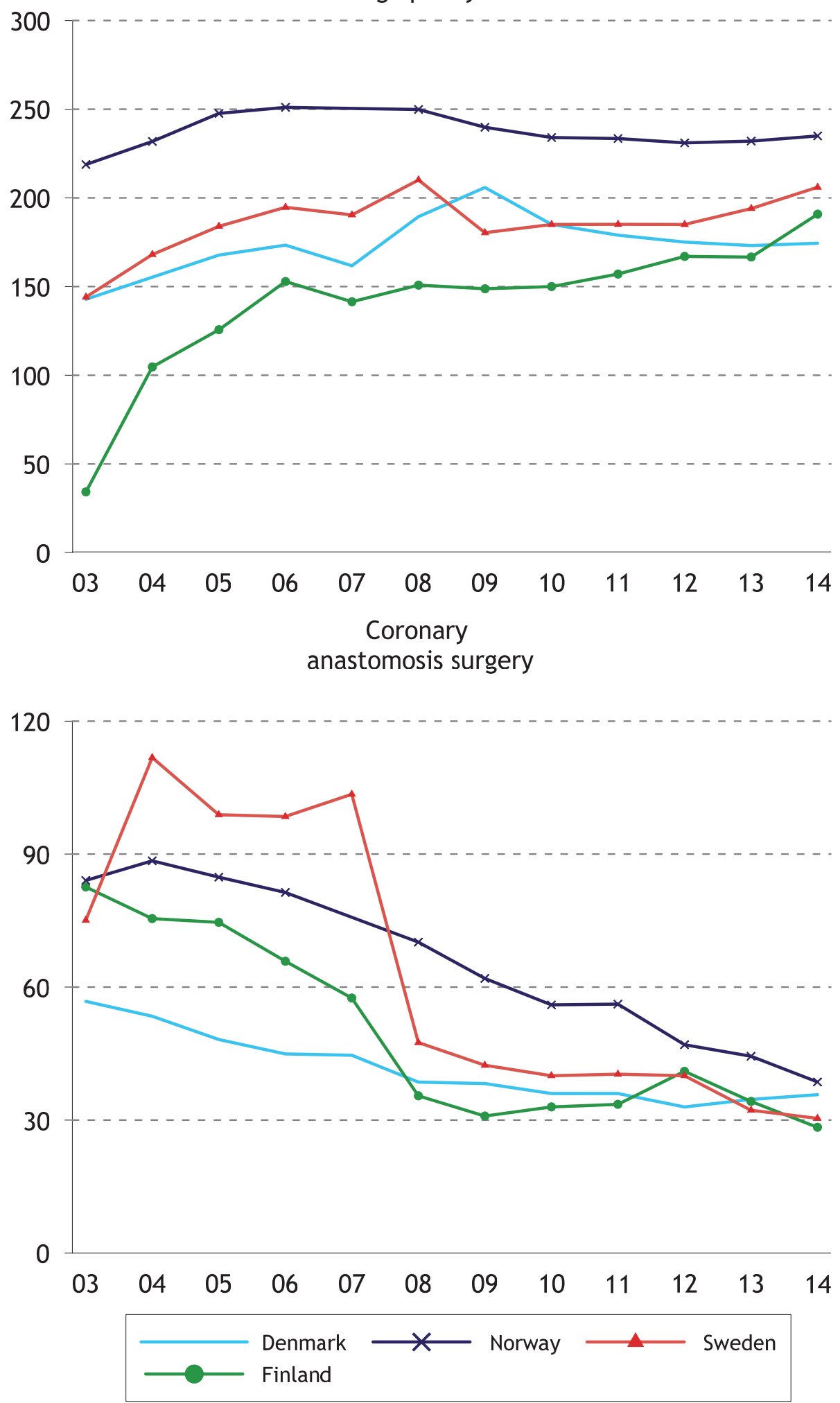

Source: The national in-patient registers 
Figure 3.5.2 Hip replacement per 100000 inhabitants, 2000-2014

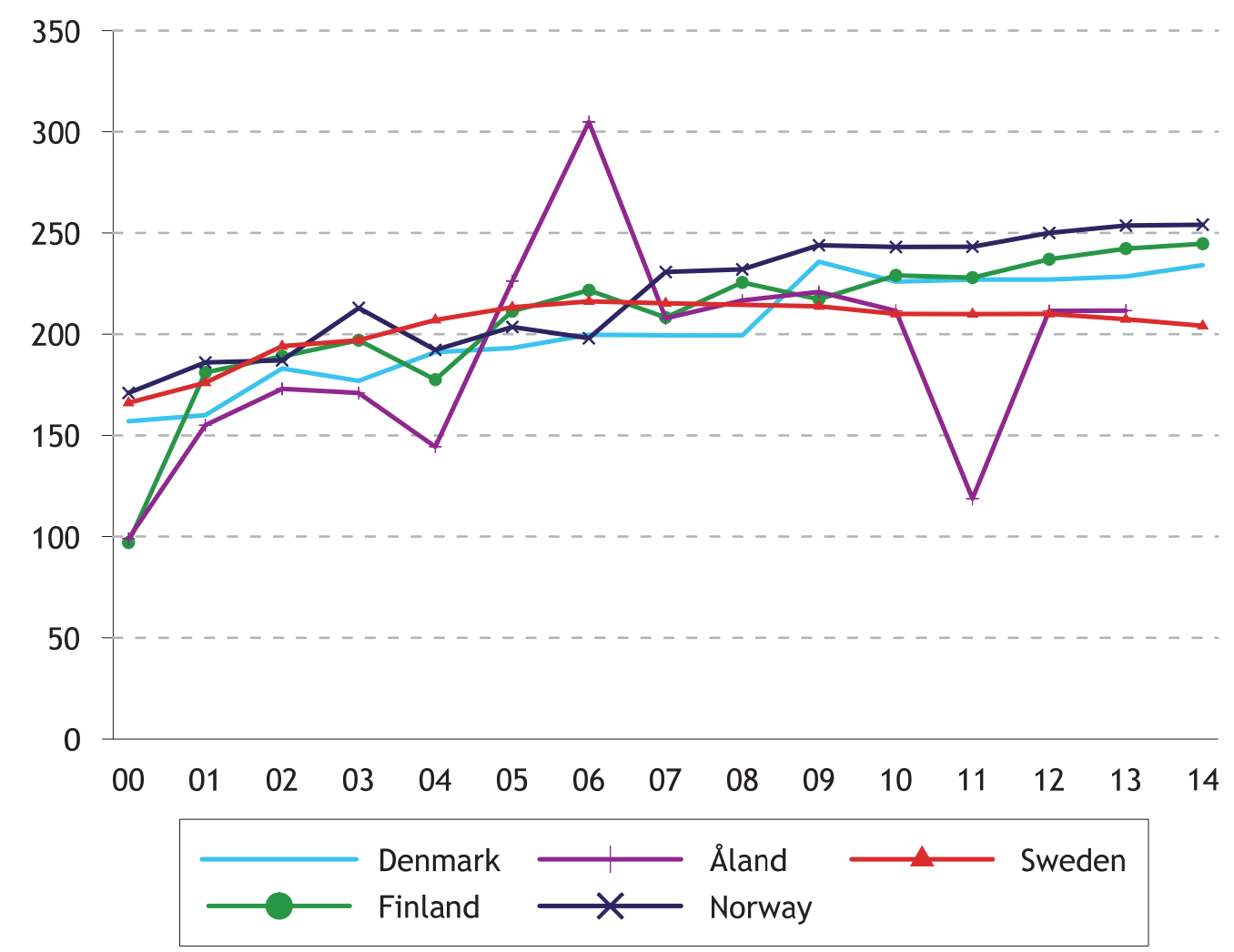

Source: The national in-patient registers 


\subsection{Accidents and self-inflicted injury}

Patients admitted to hospital because of accidents occupy a substantial part of the capacity in hospitals.

While statistics on causes of death are highly developed in the Nordic countries, registration of survivors following accidents is still incomplete, and the available data are difficult to compare. As only Denmark and Iceland have comparable statistics on external causes of accidents, it is not possible to present Nordic statistics on this.

Therefore, statistics are presented for hospital discharges for the most common serious accidents that usually require admission. The statistics show marked differences, both between countries and between men and women.

Table 3.6.1 Discharges from hospitals after treatment for injuries per 100000 inhabitants and by gender, 2014 ${ }^{1}$

\begin{tabular}{|c|c|c|c|c|c|c|c|c|c|c|c|c|}
\hline & \multicolumn{2}{|c|}{ Denmark } & \multicolumn{2}{|c|}{ Finland } & \multicolumn{2}{|c|}{ Åland ${ }^{2}$} & \multicolumn{2}{|c|}{ Iceland } & \multicolumn{2}{|c|}{ Norway } & \multicolumn{2}{|c|}{ Sweden } \\
\hline & M & W & M & W & M & W & M & W & M & W & M & W \\
\hline $\begin{array}{l}\text { Fracture of skull and intracranial injury } \\
\text { (SO2; SO6) }\end{array}$ & 183 & 110 & 299 & 190 & 177 & 129 & 100 & 38 & 230 & 154 & 175 & 119 \\
\hline Fracture at wrist and hand level (S62) & 63 & 28 & 67 & 28 & 40 & 11 & 9 & 7 & 41 & 15 & 19 & 9 \\
\hline Injury of lower leg (580-589) & 182 & 185 & 443 & 376 & 267 & 228 & 108 & 121 & 144 & 145 & 116 & 139 \\
\hline Injury of hip and thigh (S70-S79) & 187 & 352 & 248 & 424 & 149 & 202 & 134 & 246 & 152 & 314 & 165 & 306 \\
\hline Poisoning (T36-T65) & 179 & 224 & 84 & 95 & 42 & 41 & 27 & 37 & 80 & 113 & 80 & 117 \\
\hline Burn and corrosion (T2O-T32) & 15 & 7 & 33 & 16 & 19 & 8 & 15 & 11 & 24 & 13 & 13 & 6 \\
\hline
\end{tabular}

1 Including violence and self-inflicted injury

2 Average 2010-14

Source: The national in-patients registers

Table 3.6.2 Discharges from hospitals after treatment for injuries per 100000 inhabitants by age and gender, 20141

\begin{tabular}{lrrrrrrrrrr}
\hline & \multicolumn{2}{c}{ Denmark } & \multicolumn{2}{c}{ Finland } & \multicolumn{2}{c}{ Iceland } & \multicolumn{2}{c}{ Norway } & \multicolumn{2}{c}{ Sweden } \\
\cline { 2 - 12 } Age & \multicolumn{1}{c}{ M } & \multicolumn{1}{c}{ W } & \multicolumn{1}{c}{ M } & \multicolumn{1}{c}{ W } & \multicolumn{1}{c}{ M } & \multicolumn{1}{c}{ W } & M & W & \multicolumn{1}{c}{ M } & W \\
\hline $0-14$ & 427 & 366 & 1055 & 747 & 267 & 271 & 993 & 732 & 1005 & 707 \\
$15-24$ & 837 & 765 & 2135 & 1187 & 550 & 365 & 1483 & 1079 & 1277 & 1086 \\
$25-64$ & 686 & 537 & 2276 & 1420 & 671 & 519 & 1298 & 984 & 1327 & 1111 \\
$65-79^{2}$ & 1126 & 1386 & 3374 & 3526 & 1620 & 2100 & 2334 & 2685 & 4925 & 5918 \\
$80+$ & 3489 & 5193 & 8835 & 11019 & 5524 & 6630 & 5660 & 7804 &.. &.. \\
Total & 809 & 906 & 2426 & 2246 & 804 & 868 & 1520 & 1531 & 1897 & 2048 \\
\hline
\end{tabular}

1 Including violence and self-inflicted injury

2 Sweden 64+

Source: The national in-patients registers 


\subsection{Development in consumption of pharmaceutical products}

All prevalence tables are based on prescription data.

Data sources in this section: Denmark: the Danish Health Data Authority; Faroe Islands: Chief Pharmaceutical Officer; Greenland: Central Pharmacy in Copenhagen County; Finland and Åland: Finnish Medicines Agency; Iceland: Icelandic Medicines Agency; Norway: Norwegian Institute of Public Health; Sweden: Swedish eHealth Agency and National Board of Health and Welfare.

Table 3.7.1 Sales of pharmaceutical products in total, DDD/1 000 inhabitants/day by ATC-group, $2015^{1}$

\begin{tabular}{|c|c|c|c|c|c|c|c|c|}
\hline & Denmark & $\begin{array}{l}\text { Faroe } \\
\text { Islands }\end{array}$ & Greenland & Finland & Åland & Iceland & Norway & Sweden \\
\hline $\begin{array}{l}\text { A Alimentary tract } \\
\text { and metabolism }\end{array}$ & 172 & 203 & 127 & 270 & 203 & 190 & 202 & 237 \\
\hline $\begin{array}{l}\text { B Blood and blood- } \\
\text { forming organs }\end{array}$ & 115 & 132 & 63 & 144 & 142 & 155 & 122 & 259 \\
\hline $\begin{array}{l}\text { C Cardiovascular } \\
\text { system }\end{array}$ & 552 & 588 & 284 & 556 & 401 & 381 & 411 & 490 \\
\hline $\begin{array}{l}\text { G Genito-urinary } \\
\text { system }\end{array}$ & 99 & 79 & 70 & 133 & 125 & 111 & 110 & 102 \\
\hline $\begin{array}{l}\text { H Systemic hormonal } \\
\text { preparations excl. } \\
\text { sex hormones and } \\
\text { insulins }\end{array}$ & 35 & 30 & 14 & 54 & 56 & 45 & 45 & 45 \\
\hline $\begin{array}{l}\text { Anti-infectives for } \\
\text { systemic use }\end{array}$ & 21 & 18 & 23 & 22 & 18 & 24 & 21 & 16 \\
\hline $\begin{array}{l}\text { L Antineoplastic and } \\
\text { immunomodulating } \\
\text { agents }\end{array}$ & 18 & 17 & 9 & 19 & 17 & 17 & 18 & 19 \\
\hline $\begin{array}{l}\text { M Musculo-skeletal } \\
\text { system }\end{array}$ & 65 & 50 & 27 & 98 & 72 & 91 & 62 & 68 \\
\hline N Nervous system & 265 & 210 & 112 & 260 & 215 & 380 & 227 & 294 \\
\hline $\begin{array}{l}\text { P Antiparasitic prod- } \\
\text { ucts, insecticides } \\
\text { and repellents }\end{array}$ & 1 & 2 & 2 & 2 & 2 & 2 & 1 & 1 \\
\hline R Respiratory system & 133 & 118 & 54 & 163 & 139 & 140 & 197 & 172 \\
\hline S Sensory organs & 12 & 11 & 15 & 23 & 19 & 1 & 19 & 23 \\
\hline
\end{tabular}

1 Only ATC groups with WHO DDDs assigned are included. A11 Vitamins is excluded due to different definitions of pharmaceutical products in the Nordic countries 
Morbidity, Medical Treatment, Accidents and Pharmaceutical products

Table 3.7.2 Sales of drugs for acid related disorders (ATC group A02), DDD/1 000 inhabitants/day, 2005-2015

\begin{tabular}{|c|c|c|c|c|c|c|c|c|}
\hline & Denmark & $\begin{array}{c}\text { Faroe } \\
\text { Islands }\end{array}$ & Greenland & Finland & Åland & Iceland & Norway & Sweden \\
\hline \multicolumn{9}{|l|}{ A02 } \\
\hline \multicolumn{9}{|c|}{$\begin{array}{l}\text { Drugs for acid } \\
\text { related disorders }\end{array}$} \\
\hline 2005 & 38.9 & 37.6 & 18.9 & 32.7 & 30.5 & 54.2 & 32.5 & 43.1 \\
\hline 2010 & 58.8 & 58.3 & 41.9 & 55.0 & 48.9 & 84.7 & 46.5 & 61.1 \\
\hline 2013 & 68.2 & 77.8 & 45.2 & 66.0 & 56.4 & 102.4 & 54.3 & 72.2 \\
\hline 2014 & 70.9 & 83.4 & 45.3 & 68.6 & 58.3 & 106.0 & 57.0 & 74.5 \\
\hline 2015 & 74.2 & 86.7 & 49.7 & 67.7 & 63.2 & 114.5 & 58.5 & 78.4 \\
\hline \multicolumn{9}{|l|}{$\mathrm{A} 02 \mathrm{~A}$} \\
\hline \multicolumn{9}{|c|}{ Antacids } \\
\hline 2005 & 7.3 & 4.2 & 2.1 & 2.8 & 2.7 & 2.4 & 2.1 & 2.6 \\
\hline 2010 & 7.1 & 3.2 & 1.4 & 2.3 & 2.3 & 4.5 & 1.4 & 1.7 \\
\hline 2013 & 7.4 & 3.2 & 1.6 & 2.2 & 2.5 & 7.0 & 1.4 & 1.7 \\
\hline 2014 & 7.5 & 3.4 & 1.9 & 2.2 & 2.5 & 7.4 & 1.3 & 1.7 \\
\hline 2015 & 7.7 & 3.7 & 2.1 & 2.2 & 2.6 & 8.2 & 1.3 & 1.7 \\
\hline \multicolumn{9}{|l|}{$A 02 B$} \\
\hline \multicolumn{9}{|c|}{$\begin{array}{l}\text { Drugs for peptic } \\
\text { ulcer and gastro- } \\
\text { oesophageal } \\
\text { reflux disease }\end{array}$} \\
\hline 2005 & 31.6 & 33.4 & 16.8 & 29.9 & 27.8 & 51.9 & 30.4 & 40.8 \\
\hline 2010 & 51.7 & 55.1 & 40.4 & 52.7 & 46.6 & 80.2 & 45.0 & 59.5 \\
\hline 2013 & 60.8 & 74.7 & 43.5 & 63.8 & 53.9 & 95.4 & 52.9 & 70.5 \\
\hline 2014 & 63.4 & 80.0 & 43.5 & 66.4 & 55.9 & 101.1 & 55.8 & 72.9 \\
\hline 2015 & 66.5 & 83.1 & 47.6 & 65.5 & 60.6 & 106.3 & 57.3 & 76.7 \\
\hline \multicolumn{9}{|c|}{$\begin{array}{l}\text { A02BA H2-receptor } \\
\text { antagonists }\end{array}$} \\
\hline 2005 & 6.3 & 3.3 & 0.6 & 4.1 & 4.7 & 6.6 & 5.5 & 5.5 \\
\hline 2010 & 2.2 & 1.1 & 0.1 & 2.9 & 5.8 & 4.5 & 5.8 & 3.2 \\
\hline 2013 & 1.0 & 1.0 & - & 1.5 & 4.6 & 3.4 & 4.9 & 2.3 \\
\hline 2014 & 0.9 & 1.0 & - & 1.4 & 4.2 & 3.2 & 4.6 & 2.1 \\
\hline 2015 & 0.9 & 0.8 & - & 1.3 & 4.4 & 3.1 & 3.0 & 1.9 \\
\hline \multicolumn{9}{|c|}{$\mathrm{A} 02 \mathrm{BC}$} \\
\hline \multicolumn{9}{|c|}{$\begin{array}{l}\text { Proton pump } \\
\text { inhibitors }\end{array}$} \\
\hline 2005 & 24.8 & 29.0 & 15.9 & 24.3 & 21.4 & 45.2 & 24.5 & 34.2 \\
\hline 2010 & 49.1 & 53.2 & 40.2 & 48.5 & 39.2 & 75.6 & 38.8 & 55.4 \\
\hline 2013 & 59.3 & 72.8 & 43.4 & 61.1 & 47.7 & 91.9 & 47.5 & 67.3 \\
\hline 2014 & 62.0 & 78.2 & 43.4 & 63.8 & 50.0 & 94.9 & 50.6 & 69.8 \\
\hline 2015 & 65.1 & 81.4 & 47.5 & 63.2 & 54.8 & 103.2 & 53.6 & 73.7 \\
\hline \multicolumn{9}{|c|}{$\mathrm{A} 02 \mathrm{BX}$} \\
\hline \multicolumn{9}{|c|}{$\begin{array}{l}\text { Other drugs for pep- } \\
\text { tic ulcer and gastro- } \\
\text { oesophageal reflux } \\
\text { disease }\end{array}$} \\
\hline 2005 & 0.5 & 1.1 & 0.3 & 1.4 & 1.6 & - & 0.4 & 1.1 \\
\hline 2010 & 0.4 & 0.8 & - & 1.2 & 1.5 & - & 0.4 & 0.8 \\
\hline 2013 & 0.5 & 0.8 & - & 1.1 & 1.5 & - & 0.6 & 0.9 \\
\hline 2014 & 0.5 & 0.9 & - & 1.1 & 1.6 & - & 0.6 & 0.9 \\
\hline 2015 & 0.5 & 0.9 & - & 1.1 & 1.3 & - & 0.6 & 0.9 \\
\hline
\end{tabular}


Morbidity, Medical Treatment, Accidents and Pharmaceutical products

Figure 3.7.1 Sales of drugs for treatment of peptic ulcer and gastro-oesophageal reflux disease (ATC-group A02B),

DDD/1 000 inhabitants/day, 2005-2015

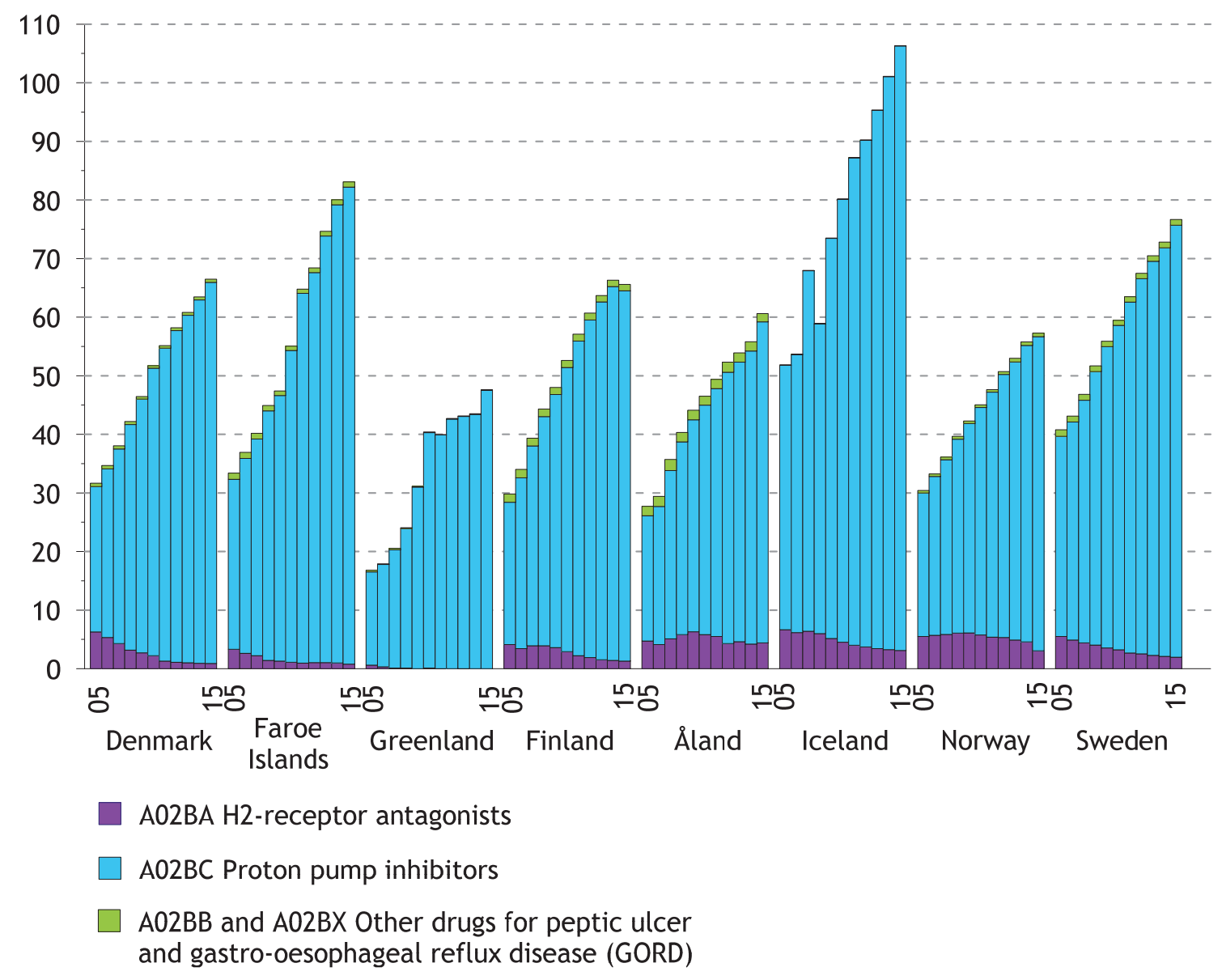


Morbidity, Medical Treatment, Accidents and Pharmaceutical products

Table 3.7.3 Proportion of the population per 1000 by age and gender (one-year prevalence), receiving at least one proton pump inhibitor (ATC group $\mathrm{A02BC}$ ) for acid related disorders, 2015

\begin{tabular}{|c|c|c|c|}
\hline & Men & Women & Total \\
\hline \multicolumn{4}{|c|}{ Denmark } \\
\hline $0-14$ & 7 & 9 & 8 \\
\hline $15-24$ & 21 & 42 & 31 \\
\hline $25-44$ & 56 & 70 & 63 \\
\hline $45-64$ & 119 & 146 & 132 \\
\hline $65-74$ & 195 & 219 & 208 \\
\hline $75+$ & 262 & 291 & 279 \\
\hline Total & .. &.. & .. \\
\hline \multicolumn{4}{|c|}{ Faroe Islands } \\
\hline $0-14$ & 7 & 4 & 6 \\
\hline $15-24$ & 19 & 30 & 24 \\
\hline $25-44$ & 61 & 67 & 64 \\
\hline $45-64$ & 133 & 145 & 139 \\
\hline $65-74$ & 257 & 291 & 273 \\
\hline $75+$ & 383 & 438 & 415 \\
\hline Total & 101 & 122 & 111 \\
\hline \multicolumn{4}{|c|}{ Finland } \\
\hline $0-14$ & 5 & 5 & .. \\
\hline $15-24$ & 19 & 35 & .. \\
\hline $25-44$ & 68 & 90 & .. \\
\hline $45-64$ & 134 & 179 & .. \\
\hline $65-74$ & 191 & 238 & .. \\
\hline $75+$ & 262 & 318 & .. \\
\hline Total & 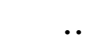 &.. & .. \\
\hline \multicolumn{4}{|c|}{ Iceland } \\
\hline $0-14$ & 19 & 21 & 20 \\
\hline $15-24$ & 38 & 73 & 55 \\
\hline $25-44$ & 75 & 95 & 85 \\
\hline $45-64$ & 160 & 216 & 188 \\
\hline $65-74$ & 256 & 357 & 315 \\
\hline $75+$ & 211 & 372 & 347 \\
\hline Total & $\cdot$ &.. & .. \\
\hline \multicolumn{4}{|c|}{ Norway } \\
\hline $0-14$ & 9 & 8 & 8 \\
\hline $15-24$ & 19 & 30 & 24 \\
\hline $25-44$ & 50 & 57 & 54 \\
\hline $45-64$ & 110 & 126 & 117 \\
\hline $65-74$ & 177 & 202 & 189 \\
\hline $75+$ & 217 & 226 & 222 \\
\hline Total & 76 & 92 & 84 \\
\hline \multicolumn{4}{|c|}{ Sweden } \\
\hline $0-14$ & 7 & 8 & 7 \\
\hline $15-24$ & 17 & 36 & 26 \\
\hline $25-44$ & 38 & 63 & 50 \\
\hline $45-64$ & 96 & 137 & 116 \\
\hline $65-74$ & 178 & 221 & 200 \\
\hline $75+$ & 258 & 296 & 280 \\
\hline Total & & & \\
\hline
\end{tabular}


Table 3.7.4 Sales of anti-obesity preparations (ATC-group A08), DDD/1 000 inhabitants/day, 2005-2015

\begin{tabular}{lcccccccc}
\hline & Denmark & $\begin{array}{c}\text { Faroe } \\
\text { Islands }\end{array}$ & Greenland & Finland & Åland & Iceland & Norway & Sweden \\
\hline 2005 & 0.7 & 0.4 & - & 0.6 & 0.3 & 1.3 & 2.6 & 2.3 \\
2012 & 0.6 & 0.6 & - & 0.3 & 0.2 & 0.1 & 0.4 & 0.5 \\
2013 & 0.5 & 0.4 & - & 0.2 & 0.2 & 0.1 & 0.4 & 0.4 \\
2014 & 0.5 & 0.3 & - & 0.2 & 0.2 & - & 0.4 & 0.4 \\
2015 & 0.4 & 0.2 & - & 0.1 & 0.1 & - & 0.4 & 0.4 \\
\hline
\end{tabular}

Table 3.7.5 Sales of drugs used in diabetes (ATC-group A10), DDD/1 000 inhabitants/day, 2005-2015

\begin{tabular}{|c|c|c|c|c|c|c|c|c|}
\hline & Denmark & $\begin{array}{l}\text { Faroe } \\
\text { Islands }\end{array}$ & $\begin{array}{l}\text { Green- } \\
\text { land }\end{array}$ & Finland & Åland & Iceland & Norway & Sweden \\
\hline \multicolumn{9}{|c|}{$\begin{array}{l}\text { A10 } \\
\text { Drugs used } \\
\text { for diabetes }\end{array}$} \\
\hline 2005 & 34.9 & 32.9 & 10.3 & 66.4 & 38.6 & 24.0 & 39.3 & 44.6 \\
\hline 2010 & 47.6 & 49.6 & 13.2 & 83.4 & 49.3 & 31.7 & 48.3 & 51.9 \\
\hline 2013 & 51.8 & 59.9 & 16.4 & 86.0 & 52.0 & 42.6 & 48.8 & 55.8 \\
\hline 2014 & 52.5 & 62.4 & 18.9 & 88.2 & 54.2 & 43.5 & 49.9 & 56.8 \\
\hline 2015 & 53.8 & 62.8 & 20.1 & 90.1 & 54.0 & 46.1 & 51.4 & 58.7 \\
\hline \multicolumn{9}{|c|}{$\begin{array}{l}\mathrm{A} 10 \mathrm{~A} \\
\text { Insulins and analogues }\end{array}$} \\
\hline 2005 & 13.3 & 10.4 & 2.7 & 21.7 & 15.1 & 6.5 & 17.4 & 22.6 \\
\hline 2010 & 17.2 & 14.2 & 3.8 & 30.0 & 19.8 & 9.8 & 19.2 & 26.4 \\
\hline 2013 & 17.8 & 13.5 & 3.9 & 31.4 & 21.0 & 11.7 & 19.1 & 27.5 \\
\hline 2014 & 18.3 & 14.3 & 4.7 & 31.8 & 21.5 & 11.6 & 19.2 & 27.8 \\
\hline 2015 & 18.6 & 14.7 & 5.1 & 31.7 & 22.0 & 12.4 & 19.4 & 28.1 \\
\hline \multicolumn{9}{|c|}{$\begin{array}{l}\text { A10AB } \\
\text { Insulins and analogues } \\
\text { for injection, fast- } \\
\text { acting }\end{array}$} \\
\hline 2005 & 4.1 & 2.9 & .. & 5.5 & 5.3 & .. & 6.0 & 8.1 \\
\hline 2010 & 5.3 & 4.8 & .. & 8.1 & 5.9 & 4.0 & 7.2 & 9.3 \\
\hline 2013 & 5.7 & 5.1 & .. & 8.8 & 6.7 & 4.7 & 7.5 & 9.7 \\
\hline 2014 & 5.8 & 5.5 & 1.0 & 9.0 & 6.9 & 4.5 & 7.6 & 9.8 \\
\hline 2015 & 5.9 & 5.9 & 1.0 & 9.3 & 7.7 & 5.0 & 7.9 & 10.0 \\
\hline \multicolumn{9}{|c|}{$\begin{array}{l}\text { A10AC } \\
\text { Insulins and analogues } \\
\text { for injection, } \\
\text { intermediate-acting }\end{array}$} \\
\hline 2005 & 4.7 & 2.8 & .. & 9.6 & 6.0 & .. & 8.2 & 5.3 \\
\hline 2010 & 3.6 & 1.0 & .. & 3.8 & 6.2 & 1.3 & 7.0 & 4.6 \\
\hline 2013 & 3.1 & 0.5 & .. & 1.8 & 7.2 & 1.4 & 6.6 & 5.2 \\
\hline 2014 & 3.0 & 0.5 & 1.6 & 1.5 & 7.5 & 1.3 & 6.6 & 5.4 \\
\hline 2015 & 2.8 & 0.5 & 2.0 & 1.2 & 7.0 & 1.1 & 6.6 & 5.4 \\
\hline \multicolumn{9}{|c|}{$\begin{array}{l}\text { A10AD } \\
\text { Insulins and analogues } \\
\text { for injection, inter- } \\
\text { mediate- or long- } \\
\text { acting combined } \\
\text { with fast-acting }\end{array}$} \\
\hline 2005 & 3.6 & 4.4 & .. & 2.8 & 1.7 & .. & 2.6 & 5.7 \\
\hline 2010 & 4.3 & 6.1 & .. & 2.3 & 2.0 & 1.8 & 2.4 & 6.5 \\
\hline 2013 & 3.6 & 4.5 & .. & 1.1 & 1.5 & 1.5 & 1.8 & 6.3 \\
\hline 2014 & 3.4 & 4.5 & 1.5 & 0.9 & 1.3 & 1.3 & 1.6 & 6.1 \\
\hline 2015 & 3.2 & 4.4 & 1.3 & 0.8 & 1.1 & 1.2 & 1.4 & 5.8 \\
\hline
\end{tabular}

The table continues 
Table 3.7.5 Sales of drugs used in diabetes (ATC-group A10), DDD/1 000 inhabitants/day, 2005-2015, continued

\begin{tabular}{|c|c|c|c|c|c|c|c|c|}
\hline & Denmark & $\begin{array}{l}\text { Faroe } \\
\text { Islands }\end{array}$ & Greenland & Finland & Åland & Iceland & Norway & Sweden \\
\hline \multicolumn{9}{|c|}{$\begin{array}{l}\text { Insulins and ana- } \\
\text { logues for injection, } \\
\text { long-acting for in- } \\
\text { jection, long-acting }\end{array}$} \\
\hline 2005 & 0.8 & 0.5 & .. & 3.9 & 2.0 & 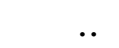 & 0.6 & 3.6 \\
\hline 2010 & 4.0 & 2.4 & .. & 15.9 & 5.6 & 2.7 & 2.6 & 6.0 \\
\hline 2013 & 5.5 & 3.4 & .. & 19.7 & 5.6 & 4.1 & 3.3 & 6.2 \\
\hline 2014 & 6.0 & 3.8 & 0.7 & 20.4 & 5.9 & 4.4 & 3.3 & 6.5 \\
\hline 2015 & 6.7 & 4.0 & .. & 20.4 & 6.2 & 5.1 & 3.5 & 6.8 \\
\hline \multicolumn{9}{|c|}{$\begin{array}{l}\text { A10B } \\
\text { Blood glucose low- } \\
\text { ering drugs, excl. } \\
\text { insulins }\end{array}$} \\
\hline 2005 & 21.6 & 22.5 & 7.6 & 44.7 & 23.5 & 17.5 & 21.9 & 22.0 \\
\hline 2010 & 30.4 & 35.5 & 9.5 & 53.4 & 29.5 & 22.0 & 29.1 & 25.5 \\
\hline 2013 & 34.0 & 46.5 & 12.5 & 54.6 & 30.9 & 30.9 & 29.7 & 28.3 \\
\hline 2014 & 34.2 & 48.1 & 14.2 & 56.4 & 32.7 & 32.0 & 30.7 & 29.0 \\
\hline 2015 & 35.2 & 48.2 & 15.0 & 58.3 & 32.0 & 33.6 & 32.0 & 30.6 \\
\hline \multicolumn{9}{|c|}{ A10BA } \\
\hline \multicolumn{9}{|c|}{ Biguanides } \\
\hline 2005 & 7.9 & 6.7 & 4.3 & 18.5 & 10.1 & 7.7 & 9.7 & 11.8 \\
\hline 2010 & 15.5 & 12.5 & 6.3 & 32.0 & 17.8 & 11.4 & 14.7 & 17.5 \\
\hline 2013 & 19.0 & 21.5 & 8.6 & 31.2 & 18.1 & 14.2 & 14.3 & 19.4 \\
\hline 2014 & 19.2 & 23.3 & 10.4 & 31.6 & 19.3 & 14.7 & 14.4 & 19.4 \\
\hline 2015 & 19.4 & 23.6 & 10.0 & 31.6 & 18.6 & 15.1 & 14.4 & 19.9 \\
\hline \multicolumn{9}{|c|}{$\begin{array}{l}\text { A10BB } \\
\text { Sulphonamides, } \\
\text { urea derivatives }\end{array}$} \\
\hline 2005 & 12.0 & 15.7 & 3.3 & 24.1 & 13.1 & 7.2 & 11.1 & 7.7 \\
\hline 2010 & 11.1 & 21.0 & 3.0 & 12.2 & 8.5 & 8.1 & 11.5 & 4.7 \\
\hline 2013 & 7.6 & 15.7 & 3.8 & 5.1 & 6.5 & 13.1 & 9.1 & 4.4 \\
\hline 2014 & 6.7 & 13.8 & 3.5 & 3.9 & 6.1 & 13.3 & 8.3 & 4.2 \\
\hline 2015 & 6.0 & 12.0 & 4.2 & 3.2 & 5.4 & 13.4 & 7.7 & 4.1 \\
\hline \multicolumn{9}{|c|}{$\begin{array}{l}\text { A10BD } \\
\text { Combinations of } \\
\text { oral blood glucose } \\
\text { lowering }\end{array}$} \\
\hline 2005 & 0.2 &.. & - & 0.8 & 0.1 & 0.5 & 0.1 & 0.2 \\
\hline 2010 & 1.1 & - & 0.1 & 3.0 & 0.3 & 0.3 & 1.1 & 0.4 \\
\hline 2013 & 1.8 & 0.1 & - & 5.2 & 0.1 & 0.7 & 2.5 & 0.3 \\
\hline 2014 & 2.2 & 0.1 & - & 5.6 & 0.2 & 0.8 & 3.0 & 0.4 \\
\hline 2015 & 2.5 & 0.2 & - & 5.8 & 0.2 & 1.0 & 3.4 & 0.4 \\
\hline \multicolumn{9}{|c|}{ A10BG } \\
\hline 2005 & 0.1 & 0.1 & - & 1.1 & 0.1 & 1.7 & 0.8 & 1.0 \\
\hline 2010 & 0.1 & - & - & 1.8 & 1.9 & 0.7 & 0.6 & 0.6 \\
\hline 2013 & - & - & - & 1.0 & 1.2 & 0.4 & 0.3 & 0.3 \\
\hline 2014 & - & - & - & 1.0 & 1.2 & 0.4 & 0.2 & 0.3 \\
\hline 2015 & - & - & - & 0.9 & 1.0 & 0.3 & 0.2 & 0.3 \\
\hline
\end{tabular}


Table 3.7.5 Sales of drugs used in diabetes (ATC-group A10), DDD/1 000 inhabitants/day, 2005-2015, continued

\begin{tabular}{|c|c|c|c|c|c|c|c|c|}
\hline & Denmark & $\begin{array}{l}\text { Faroe } \\
\text { Islands }\end{array}$ & Greenland & Finland & Åland & Iceland & Norway & Sweden \\
\hline \multicolumn{9}{|c|}{ A10BH } \\
\hline \multirow{2}{*}{\multicolumn{9}{|c|}{$\begin{array}{l}\text { Dipeptidyl peptidase } \\
4 \text { (DPP-4) inhibitors }\end{array}$}} \\
\hline & & & & & & & & \\
\hline 2005 & & & - & . & .. & - & - & 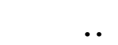 \\
\hline 2010 & 1.2 & 0.7 & - & 4.0 & 0.6 & 1.0 & 0.9 & 0.9 \\
\hline 2013 & 2.0 & 3.6 & - & 10.4 & 4.5 & 1.6 & 2.1 & 1.9 \\
\hline 2014 & 2.3 & 4.0 & - & 11.8 & 5.4 & 1.6 & 2.5 & 2.3 \\
\hline 2015 & 2.6 & 4.7 & - & 13.4 & 5.8 & 2.0 & 3.0 & 2.9 \\
\hline \multicolumn{9}{|c|}{$\begin{array}{l}\text { A10BX } \\
\text { Other oral blood } \\
\text { glucose lowering } \\
\text { drugs, excl. insulins }\end{array}$} \\
\hline 2005 & 0.3 & - & - & 0.2 & 0.2 & 0.4 & 0.1 & 1.2 \\
\hline 2010 & 1.3 & 1.3 & - & 0.5 & 0.5 & 0.3 & 0.2 & 1.3 \\
\hline 2013 & 3.5 & 5.6 & 0.1 & 1.8 & 0.5 & 1.0 & 1.4 & 2.0 \\
\hline 2014 & 3.9 & 7.0 & 0.2 & 2.6 & 0.6 & 1.3 & 2.3 & 2.4 \\
\hline 2015 & 4.8 & 7.7 & 0.8 & 2.0 & 1.0 & 1.9 & 3.3 & 3.0 \\
\hline
\end{tabular}

Figure 3.7.2 Sales of insulins and other blood glucose lowering drugs (ATC-groups $A 10 A$ and $A 10 B), D D D / 1000$ inhabitants/day, 2005-2015

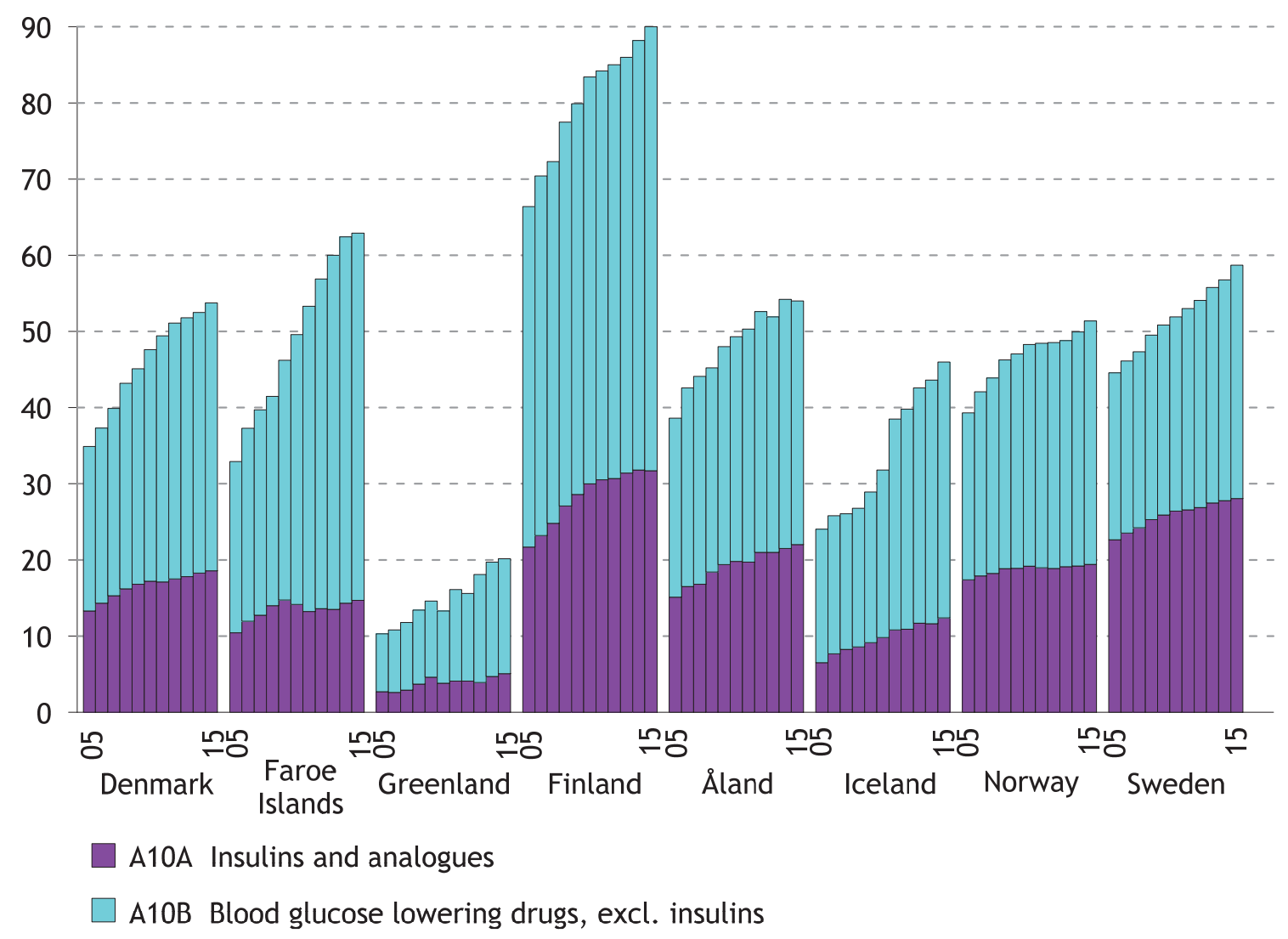


Morbidity, Medical Treatment, Accidents and Pharmaceutical products

Table 3.7.6 Proportion of the population per 1000 by age and gender (one-year prevalence) receiving at least one drug used in diabetes (ATC-group A10), 2015

\begin{tabular}{|c|c|c|c|}
\hline & Men & Women & Total \\
\hline \multicolumn{4}{|c|}{ Denmark } \\
\hline $0-14$ & 2 & 2 & 2 \\
\hline $15-24$ & 5 & 7 & 6 \\
\hline $25-44$ & 14 & 16 & 15 \\
\hline $45-64$ & 69 & 46 & 57 \\
\hline $65-74$ & 140 & 89 & 114 \\
\hline $75+$ & 152 & 106 & 125 \\
\hline Total & .. &.. & .. \\
\hline \multicolumn{4}{|c|}{ Faroe Islands } \\
\hline $0-14$ & 1 & 1 & 1 \\
\hline $15-24$ & 5 & 8 & 6 \\
\hline $25-44$ & 13 & 16 & 15 \\
\hline $45-64$ & 62 & 38 & 50 \\
\hline $65-74$ & 180 & 101 & 143 \\
\hline $75+$ & 224 & 126 & 167 \\
\hline Total & 51 & 35 & 43 \\
\hline \multicolumn{4}{|c|}{ Finland } \\
\hline $0-14$ & 5 & 4 & .. \\
\hline $15-24$ & 11 & 10 & .. \\
\hline $25-44$ & 19 & 18 & .. \\
\hline $45-64$ & 99 & 65 & .. \\
\hline $65-74$ & 210 & 140 & .. \\
\hline $75+$ & 225 & 178 & .. \\
\hline Total & .. &.. & .. \\
\hline \multicolumn{4}{|c|}{ Iceland } \\
\hline $0-14$ & 2 & 2 & 2 \\
\hline $15-24$ & 8 & 14 & 11 \\
\hline $25-44$ & 19 & 71 & 25 \\
\hline $45-64$ & 102 & 67 & 84 \\
\hline $65-74$ & 205 & 122 & 171 \\
\hline $75+$ & 238 & 125 & 174 \\
\hline Total & .. &.. & .. \\
\hline \multicolumn{4}{|c|}{ Norway } \\
\hline $0-14$ & 2 & 2 & 2 \\
\hline $15-24$ & 6 & 7 & 7 \\
\hline $25-44$ & 14 & 14 & 14 \\
\hline $45-64$ & 59 & 39 & 49 \\
\hline $65-74$ & 119 & 76 & 97 \\
\hline $75+$ & 120 & 85 & 99 \\
\hline Total & 39 & 30 & 35 \\
\hline \multicolumn{4}{|c|}{ Sweden } \\
\hline $0-14$ & 3 & 3 & 3 \\
\hline $15-24$ & 8 & 8 & 8 \\
\hline $25-44$ & 14 & 12 & 13 \\
\hline $45-64$ & 68 & 42 & 55 \\
\hline $65-74$ & 155 & 96 & 125 \\
\hline $75+$ & 174 & 121 & 143 \\
\hline Total & .. & 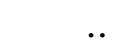 & \\
\hline
\end{tabular}


Table 3.7.7 Sales of antithrombotic agents (ATC-group B01), DDD/1 000 inhabitants/day, 2005-2015

\begin{tabular}{|c|c|c|c|c|c|c|c|c|}
\hline & Denmark & $\begin{array}{l}\text { Faroe } \\
\text { Islands }\end{array}$ & Greenland & Finland & Åland & Iceland & Norway & Sweden \\
\hline \multicolumn{9}{|l|}{ B01A } \\
\hline \multicolumn{9}{|c|}{ Antithrombotic agents } \\
\hline 2005 & 85.6 & 52.8 & .. & 124.7 & 86.0 & .. & 80.4 & 85.6 \\
\hline 2010 & 99.5 & 75.2 & 42.0 & 118.7 & 85.2 & 81.2 & 95.6 & 93.5 \\
\hline 2013 & 98.5 & 83.9 & 47.8 & 121.6 & 87.6 & 85.1 & 98.5 & 92.4 \\
\hline 2014 & 97.1 & 86.7 & 39.7 & 117.9 & 88.7 & 86.9 & 97.4 & 90.5 \\
\hline 2015 & 96.4 & 84.0 & 47.2 & 118.4 & 91.4 & 87.0 & 96.5 & 89.5 \\
\hline \multicolumn{9}{|c|}{$\mathrm{B} 01 \mathrm{AA}$} \\
\hline \multicolumn{9}{|c|}{ Vitamin $\mathrm{K}$ antagonists } \\
\hline 2005 & 6.4 & 6.1 & .. & 10.7 & 17.9 & .. & 10.3 & 7.5 \\
\hline 2010 & 8.1 & 7.3 & 3.2 & 13.9 & 14.4 & 8.0 & 11.2 & 9.3 \\
\hline 2013 & 8.6 & 7.4 & 2.1 & 17.0 & 16.1 & 7.7 & 9.2 & 11.4 \\
\hline 2014 & 8.6 & 6.9 & 2.1 & 17.0 & 16.1 & 7.7 & 9.2 & 11.4 \\
\hline 2015 & 8.5 & 6.3 & 2.3 & 17.6 & 16.5 & 7.2 & 8.0 & 10.6 \\
\hline \multicolumn{9}{|c|}{ B01AB } \\
\hline \multicolumn{9}{|c|}{ Heparin group } \\
\hline 2005 & 2.0 & 1.2 & .. & 3.2 & 3.1 & .. & 3.6 & 3.6 \\
\hline 2010 & 2.5 & 1.2 & 0.7 & 5.3 & 5.6 & 2.6 & 5.1 & 5.3 \\
\hline 2013 & 3.2 & 2.3 & 1.0 & 6.5 & 5.4 & 3.1 & 6.1 & 6.1 \\
\hline 2014 & 3.5 & 3.3 & 1.3 & 6.9 & 5.4 & 3.3 & 5.9 & 6.2 \\
\hline 2015 & 3.5 & 3.1 & 1.1 & 7.3 & 7.1 & 3.6 & 6.0 & 6.2 \\
\hline \multicolumn{9}{|c|}{$\mathrm{B} 01 \mathrm{AC}$} \\
\hline \multicolumn{9}{|c|}{$\begin{array}{l}\text { Platelet aggregation } \\
\text { inhibitors excl. heparin }\end{array}$} \\
\hline 2005 & 77.1 & 45.5 & 31.8 & 110.7 & 65.1 & 65.1 & 66.5 & 74.4 \\
\hline 2010 & 88.8 & 66.5 & 38.1 & 99.3 & 65.2 & 70.7 & 79.3 & 78.8 \\
\hline 2013 & 83.5 & 68.4 & 41.2 & 97.8 & 66.5 & 72.1 & 78.3 & 73.7 \\
\hline 2014 & 80.1 & 66.5 & 32.0 & 92.4 & 67.0 & 72.7 & 76.5 & 70.1 \\
\hline 2015 & 77.5 & 63.9 & 39.7 & 90.8 & 67.3 & 70.9 & 74.5 & 67.0 \\
\hline \multicolumn{9}{|c|}{ B01AE } \\
\hline \multicolumn{9}{|c|}{$\begin{array}{l}\text { Direct thrombin } \\
\text { inhibitors }\end{array}$} \\
\hline 2005 & - & .. & .. & - & .. & 0.1 & - & - \\
\hline 2012 & 1.7 & 1.2 & 0.5 & 0.3 & 0.1 & 0.7 & 0.4 & 0.3 \\
\hline 2013 & 2.7 & 1.7 & 2.3 & 0.5 & 0.1 & 1.3 & 1.7 & 0.6 \\
\hline 2014 & 3.6 & 2.1 & 4.2 & 0.7 & 0.2 & 1.6 & 2.0 & 0.9 \\
\hline 2015 & 2.7 & 1.7 & 4.0 & 1.0 & 0.3 & 1.6 & 1.9 & 1.0 \\
\hline \multicolumn{9}{|c|}{$\mathrm{B} 01 \mathrm{AF}$} \\
\hline \multicolumn{9}{|c|}{$\begin{array}{l}\text { Direct factor } \mathrm{Xa} \\
\text { inhibitors }\end{array}$} \\
\hline 2005 & - & .. & .. & .. &.. & .. & .. & .. \\
\hline 2012 & 0.2 & 0.9 & .. & 0.1 & - & 0.1 & 0.1 & - \\
\hline 2013 & 1.0 & 4.1 &.. & 0.3 & - & 0.5 & 1.9 & 0.5 \\
\hline 2014 & 2.2 & 8.0 & - & 0.7 & 0.1 & 1.6 & 3.7 & 1.9 \\
\hline 2015 & 4.2 & 8.9 & 0.1 & 1.7 & 0.3 & 3.6 & 6.1 & 4.8 \\
\hline
\end{tabular}


Figure 3.7.3 Sales of cardiovascular drugs (ATC-group C), DDD/1 000 inhabitants/day, 2005-2015

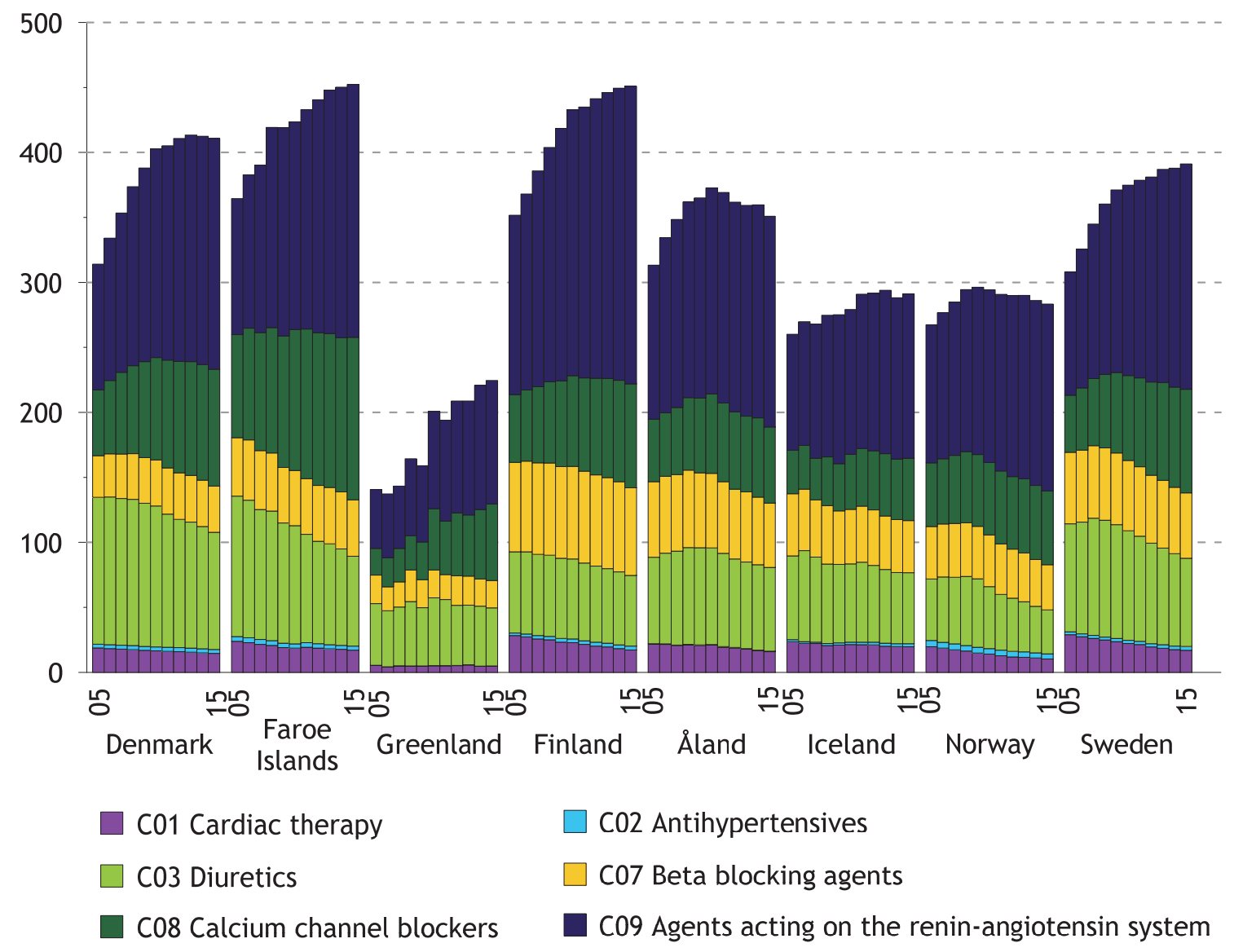


Table 3.7.8 Sales of drugs for cardiac therapy (ATC-group C01), DDD/1 000 inhabitants/day, 2005-2015

\begin{tabular}{|c|c|c|c|c|c|c|c|c|}
\hline & $\begin{array}{l}\text { Den- } \\
\text { mark }\end{array}$ & $\begin{array}{l}\text { Faroe } \\
\text { Islands }\end{array}$ & $\begin{array}{l}\text { Green- } \\
\text { land }\end{array}$ & Finland & Åland & Iceland & Norway & Sweden \\
\hline \multicolumn{9}{|l|}{$\mathrm{CO1}$} \\
\hline \multicolumn{9}{|c|}{ Cardiac therapy } \\
\hline 2005 & 18.8 & 23.9 & 5.3 & 28.3 & 21.6 & 23.7 & 19.6 & 29.0 \\
\hline 2010 & 16.5 & 18.6 & 5.0 & 22.7 & 21.0 & 21.4 & 14.0 & 22.2 \\
\hline 2013 & 15.5 & 18.1 & 5.7 & 19.5 & 18.0 & 20.1 & 11.6 & 18.6 \\
\hline 2014 & 15.1 & 17.7 & 4.7 & 18.2 & 16.7 & 19.7 & 11.0 & 17.5 \\
\hline 2015 & 14.6 & 17.2 & 4.7 & 17.3 & 15.9 & 19.6 & 10.3 & 16.9 \\
\hline \multicolumn{9}{|l|}{ C01A } \\
\hline \multicolumn{9}{|c|}{ Cardiac glycosides } \\
\hline 2005 & 6.0 & 3.7 & 1.9 & 6.0 & 5.4 & 3.0 & 4.1 & 5.9 \\
\hline 2010 & 4.7 & 2.8 & 1.5 & 4.2 & 4.9 & 2.6 & 2.4 & 3.5 \\
\hline 2013 & 4.1 & 2.6 & 1.6 & 3.5 & 4.5 & 2.5 & 1.8 & 2.8 \\
\hline 2014 & 3.9 & 2.4 & 1.2 & 3.2 & 3.8 & 2.5 & 1.5 & 2.6 \\
\hline 2015 & 3.7 & 2.5 & 1.1 & 2.9 & 3.9 & 2.5 & 1.3 & 2.4 \\
\hline \multicolumn{9}{|l|}{ C01B } \\
\hline \multicolumn{9}{|c|}{$\begin{array}{l}\text { Antiarrhytmics, } \\
\text { class I and III }\end{array}$} \\
\hline 2005 & 1.6 & 1.3 & .. & 1.7 & 1.9 & 3.4 & 1.4 & 1.1 \\
\hline 2010 & 1.5 & 1.1 & 0.5 & 1.9 & 2.3 & 3.4 & 1.8 & 1.2 \\
\hline 2013 & 1.6 & 1.1 & 0.5 & 2.1 & 2.5 & 4.2 & 2.0 & 1.4 \\
\hline 2014 & 1.6 & 1.2 & 0.6 & 2.1 & 2.6 & 4.1 & 2.0 & 1.4 \\
\hline 2015 & 1.5 & 1.2 & 0.5 & 2.1 & 2.8 & 4.3 & 2.2 & 1.4 \\
\hline \multirow{2}{*}{\multicolumn{9}{|c|}{$\begin{array}{l}\text { C01D } \\
\text { Vasodilators used in } \\
\text { cardiac diseases }\end{array}$}} \\
\hline & & & & & & & & \\
\hline 2005 & 10.5 & 18.6 & 2.8 & 19.5 & 13.1 & 17.2 & 14.0 & 21.6 \\
\hline 2010 & 9.4 & 14.3 & 2.5 & 15.5 & 12.8 & 15.2 & 9.5 & 17.1 \\
\hline 2013 & 8.9 & 13.9 & 3.3 & 12.8 & 10.2 & 13.2 & 7.4 & 14.0 \\
\hline 2014 & 8.8 & 13.7 & 2.7 & 12.1 & 9.7 & 12.9 & 7.0 & 13.1 \\
\hline 2015 & 8.5 & 13.2 & 2.7 & 11.4 & 8.4 & 12.4 & 6.4 & 12.6 \\
\hline
\end{tabular}


Table 3.7.9 Sales of cardiovascular drugs (ATC-group C02; C03; C07; C08; C09), DDD/1 000 inhabitants/day, 2005-2015

\begin{tabular}{|c|c|c|c|c|c|c|c|c|}
\hline & Denmark & $\begin{array}{l}\text { Faroe } \\
\text { Islands }\end{array}$ & Greenland & Finland & Åland & Iceland & Norway & Sweden \\
\hline \multicolumn{9}{|l|}{$\mathrm{CO2}$} \\
\hline \multicolumn{9}{|c|}{ Antihypertensives } \\
\hline $2005^{\circ}$ & 2.9 & 3.6 & 0.1 & 1.9 & 0.3 & 1.4 & 4.9 & 2.1 \\
\hline 2010 & 3.0 & 3.3 & 0.1 & 2.9 & 0.4 & 1.8 & 4.3 & 2.5 \\
\hline 2013 & 3.1 & 3.2 & 0.1 & 2.9 & 0.3 & 2.3 & 4.2 & 2.7 \\
\hline 2014 & 3.1 & 3.1 & 0.1 & 2.9 & 0.3 & 2.3 & 4.0 & 2.8 \\
\hline 2015 & 3.0 & 3.1 & 0.1 & 2.9 & 0.4 & 2.4 & 4.0 & 3.0 \\
\hline \multicolumn{9}{|c|}{$\begin{array}{l}\text { C03 } \\
\text { Diuretics }\end{array}$} \\
\hline 2005 & 112.9 & 108.0 & 47.5 & 62.5 & 66.5 & 64.5 & 47.4 & 83.0 \\
\hline 2010 & 108.5 & 91.0 & 52.1 & 61.6 & 74.3 & 60.3 & 47.5 & 84.2 \\
\hline 2013 & 97.0 & 77.7 & 45.9 & 57.4 & 66.6 & 56.7 & 38.5 & 74.3 \\
\hline 2014 & 94.0 & 74.2 & 46.1 & 56.1 & 65.8 & 52.7 & 35.7 & 70.9 \\
\hline 2015 & 90.1 & 69.2 & 44.8 & 54.3 & 64.5 & 54.6 & 33.7 & 67.9 \\
\hline \multicolumn{9}{|c|}{$\begin{array}{l}\text { C03A } \\
\text { Low-ceiling diuretics, } \\
\text { thiazides }\end{array}$} \\
\hline 2005 & 49.1 & 53.6 & 29.5 & 1.7 & 1.9 & 8.8 & 9.0 & 13.0 \\
\hline 2010 & 49.2 & 43.9 & 37.5 & 1.9 & 2.3 & 6.3 & 11.8 & 25.2 \\
\hline 2013 & 41.1 & 33.7 & 33.8 & 6.8 & 8.3 & 6.0 & 7.2 & 22.8 \\
\hline 2014 & 38.3 & 31.3 & 36.9 & 2.1 & 2.6 & 5.5 & 6.5 & 21.7 \\
\hline 2015 & 35.9 & 28.9 & 33.5 & 6.5 & 7.5 & 5.0 & 5.9 & 20.4 \\
\hline \multicolumn{9}{|c|}{$\begin{array}{l}\mathrm{CO} C \\
\text { High-ceiling diuretics }\end{array}$} \\
\hline 2005 & 53.5 & 39.6 & 15.9 & 33.5 & 25.9 & 21.2 & 30.1 & 50.7 \\
\hline 2010 & 50.9 & 36.8 & 12.8 & 37.8 & 31.7 & 23.7 & 28.4 & 42.8 \\
\hline 2013 & 48.3 & 35.5 & 9.5 & 37.1 & 30.3 & 23.1 & 25.5 & 38.3 \\
\hline 2014 & 48.5 & 35.0 & 7.1 & 37.1 & 30.7 & 22.7 & 23.9 & 36.9 \\
\hline 2015 & 47.2 & 32.8 & 9.3 & 36.1 & 31.4 & 23.7 & 22.8 & 35.8 \\
\hline \multicolumn{9}{|c|}{$\begin{array}{l}\text { CO3E } \\
\text { Diuretics and potassium- } \\
\text { sparing agents } \\
\text { in combination }\end{array}$} \\
\hline 2005 & 5.5 & 1.0 & 0.1 & 20.7 & 33.3 & 32.6 & 6.7 & 13.5 \\
\hline 2010 & 4.1 & 0.6 & 0.2 & 14.1 & 30.6 & 28.0 & 5.9 & 11.6 \\
\hline 2013 & 3.3 & 0.7 & 0.3 & 10.6 & 25.2 & 24.8 & 4.2 & 9.0 \\
\hline 2014 & 3.0 & 0.6 & - & 9.7 & 24.0 & 23.6 & 3.8 & 8.1 \\
\hline 2015 & 2.8 & 0.5 & 0.1 & 8.9 & 22.4 & 22.8 & 3.4 & 7.4 \\
\hline \multicolumn{9}{|l|}{$\mathrm{CO} 7$} \\
\hline \multicolumn{9}{|c|}{ Beta blocking agents } \\
\hline 2005 & 32.1 & 44.9 & 22.0 & 68.9 & 58.1 & 47.8 & 40.4 & 55.1 \\
\hline 2010 & 35.4 & 42.4 & 21.7 & 71.3 & 57.4 & 41.9 & 39.8 & 54.1 \\
\hline 2013 & 35.9 & 43.3 & 22.3 & 70.1 & 54.0 & 41.1 & 37.4 & 52.2 \\
\hline 2014 & 35.7 & 43.9 & 20.8 & 69.4 & 51.9 & 40.7 & 36.1 & 51.1 \\
\hline 2015 & 35.5 & 43.3 & 21.0 & 67.5 & 47.7 & 40.2 & 34.7 & 50.4 \\
\hline \multicolumn{9}{|c|}{$\begin{array}{l}\text { C08 } \\
\text { Calcium channel blockers }\end{array}$} \\
\hline 2005 & 50.7 & 79.3 & 20.5 & 52.2 & 48.1 & 33.6 & 48.9 & 44.0 \\
\hline 2010 & 78.9 & 108.5 & 47.1 & 69.7 & 61.1 & 42.2 & 55.8 & 65.5 \\
\hline 2013 & 87.6 & 118.4 & 47.0 & 76.2 & 58.2 & 48.0 & 57.2 & 75.2 \\
\hline 2014 & 89.0 & 118.6 & 53.6 & 78.1 & 61.0 & 46.5 & 56.9 & 77.1 \\
\hline 2015 & 90.0 & 125.0 & 59.0 & 80.0 & 58.5 & 48.0 & 56.9 & 79.6 \\
\hline
\end{tabular}


Table 3.7.9 Sales of cardiovascular drugs (ATC-group C02; C03; C07; C08; C09), DDD/1 000 inhabitants/day, 2005-2015, continued

\begin{tabular}{|c|c|c|c|c|c|c|c|c|}
\hline & Denmark & $\begin{array}{l}\text { Faroe } \\
\text { Islands }\end{array}$ & Greenland & Finland & Åland & Iceland & Norway & Sweden \\
\hline \multicolumn{9}{|c|}{$\begin{array}{l}\text { C08C } \\
\text { Selective calcium channel } \\
\text { blockers with mainly } \\
\text { vascular effects }\end{array}$} \\
\hline 2005 & 43.8 & 75.8 & 19.3 & 47.6 & 46.4 & 27.4 & 43.8 & 39.8 \\
\hline 2010 & 74.0 & 106.3 & 46.5 & 66.9 & 59.5 & 37.0 & 52.2 & 62.8 \\
\hline 2013 & 83.7 & 116.4 & 46.2 & 74.1 & 56.9 & 43.3 & 54.3 & 73.1 \\
\hline 2014 & 85.4 & 116.7 & 52.7 & 76.1 & 59.7 & 41.9 & 54.4 & 75.2 \\
\hline 2015 & 86.6 & 123.2 & 58.2 & 78.2 & 57.1 & 43.7 & 54.6 & 77.9 \\
\hline \multicolumn{9}{|c|}{$\begin{array}{l}\text { C08D } \\
\text { Selective calcium channel } \\
\text { blockers with direct cardiac } \\
\text { effects }\end{array}$} \\
\hline 2005 & 6.8 & 3.5 & 1.2 & 4.6 & 1.7 & 6.2 & 5.1 & 4.1 \\
\hline 2010 & 5.0 & 2.2 & 0.7 & 2.8 & 1.6 & 5.4 & 3.6 & 2.7 \\
\hline 2013 & 3.9 & 2.0 & 0.0 & 2.1 & 1.3 & 4.8 & 2.8 & 2.0 \\
\hline 2014 & 3.6 & 1.9 & 0.9 & 2.0 & 1.3 & 4.6 & 2.5 & 1.9 \\
\hline 2015 & 3.4 & 1.9 & 0.7 & 1.8 & 1.4 & 4.3 & 2.3 & 1.7 \\
\hline \multicolumn{9}{|c|}{$\begin{array}{l}\text { C09 } \\
\text { Agents acting on the } \\
\text { renin-angiotensin system }\end{array}$} \\
\hline 2005 & 96.8 & 104.7 & 45.2 & 137.9 & 118.6 & 89.2 & 106.2 & 94.7 \\
\hline 2010 & 160.5 & 159.8 & 74.8 & 204.5 & 158.4 & 111.3 & 132.9 & 146.4 \\
\hline 2013 & 174.3 & 187.3 & 87.7 & 219.9 & 162.1 & 125.7 & 141.1 & 164.0 \\
\hline 2014 & 175.7 & 192.8 & 95.5 & 224.8 & 163.9 & 124.2 & 142.4 & 168.4 \\
\hline 2015 & 177.5 & 194.6 & 95.0 & 228.9 & 162.1 & 126.4 & 143.8 & 173.4 \\
\hline \multicolumn{9}{|c|}{$\begin{array}{l}\text { CO9A } \\
\text { ACE-inhibitors, plain }\end{array}$} \\
\hline 2005 & 55.5 & 68.2 & 41.3 & 75.3 & 79.9 & 32.2 & 42.9 & 57.3 \\
\hline 2010 & 90.9 & 104.2 & 64.3 & 104.5 & 86.2 & 38.3 & 45.2 & 83.1 \\
\hline 2013 & 91.3 & 117.6 & 74.4 & 103.8 & 76.9 & 42.2 & 45.0 & 84.1 \\
\hline 2014 & 89.4 & 118.8 & 79.8 & 103.8 & 74.1 & 41.8 & 44.4 & 82.6 \\
\hline 2015 & 87.8 & 117.3 & 76.8 & 103.5 & 70.9 & 40.6 & 43.9 & 81.4 \\
\hline \multicolumn{9}{|c|}{$\begin{array}{l}\text { CO9B } \\
\text { ACE-inhibitors, } \\
\text { combinations }\end{array}$} \\
\hline 2005 & 6.7 & 5.3 & 0.1 & 14.7 & 4.2 & 7.7 & 7.3 & 3.6 \\
\hline 2010 & 19.2 & 11.9 & 0.1 & 16.4 & 5.1 & 11.0 & 6.6 & 8.2 \\
\hline 2013 & 19.0 & 14.2 & 0.1 & 14.5 & 5.5 & 5.9 & 6.2 & 8.7 \\
\hline 2014 & 18.3 & 15.3 & - & 13.9 & 5.3 & 6.1 & 5.8 & 8.7 \\
\hline 2015 & 17.6 & 15.3 & 0.1 & 13.4 & 5.0 & 6.4 & 5.5 & 8.5 \\
\hline \multicolumn{9}{|c|}{$\begin{array}{l}\text { C09C } \\
\text { Angiotensin II } \\
\text { antagonists }\end{array}$} \\
\hline 2005 & 22.1 & 20.7 & 3.8 & 31.0 & 27.8 & 23.8 & 30.6 & 24.6 \\
\hline 2010 & 32.1 & 33.4 & 10.2 & 54.7 & 53.1 & 30.6 & 44.1 & 41.2 \\
\hline 2013 & 41.9 & 44.7 & 13.0 & 70.5 & 60.8 & 37.4 & 50.3 & 55.2 \\
\hline 2014 & 45.0 & 47.4 & 15.4 & 75.5 & 65.3 & 37.1 & 52.3 & 60.4 \\
\hline 2015 & 48.7 & 49.9 & 18.0 & 80.5 & 67.4 & 39.4 & 54.7 & 66.1 \\
\hline
\end{tabular}


Table 3.7.9 Sales of cardiovascular drugs (ATC-group C02; C03; C07; C08; C09), DDD/1 000 inhabitants/day, 2005-2015, continued

\begin{tabular}{|c|c|c|c|c|c|c|c|c|}
\hline & Denmark & $\begin{array}{l}\text { Faroe } \\
\text { Islands }\end{array}$ & Greenland & Finland & Åland & Iceland & Norway & Sweden \\
\hline \multicolumn{9}{|c|}{$\begin{array}{l}\text { C09D } \\
\text { Angiotensin II antagonists, } \\
\text { combinations }\end{array}$} \\
\hline 2005 & 12.5 & 10.5 & 0.1 & 16.8 & 6.7 & 25.5 & 25.4 & 9.1 \\
\hline 2010 & 17.3 & 9.8 & 0.1 & 28.9 & 14.0 & 31.1 & 36.9 & 14.0 \\
\hline 2013 & 21.8 & 10.6 & 0.2 & 31.1 & 18.9 & 39.8 & 39.7 & 16.1 \\
\hline 2014 & 22.7 & 11.1 & 0.2 & 31.6 & 19.1 & 38.6 & 38.9 & 16.7 \\
\hline 2015 & 23.2 & 12.0 & 0.2 & 31.5 & 18.7 & 39.9 & 39.7 & 17.4 \\
\hline \multicolumn{9}{|c|}{$\begin{array}{l}\text { C09X } \\
\text { Other agents acting on the } \\
\text { rennin-angiotensin system }\end{array}$} \\
\hline 2005 & - & - & - & - & - & - & - & - \\
\hline 2010 & 1.0 & 0.5 & 0.1 & - & - & 0.3 & - & - \\
\hline 2013 & 0.3 & 0.2 & - & - & - & 0.3 & - & - \\
\hline 2014 & 0.2 & 0.2 & - & - & - & 0.2 & - & - \\
\hline 2015 & 0.2 & 0.2 & - & - & - & 0.2 & - & - \\
\hline
\end{tabular}

Table 3.7.10 Sales of serum lipid modifying agents (ATC-group C10), DDD/1 000 inhabitants/day, 2005-2015

\begin{tabular}{|c|c|c|c|c|c|c|c|c|}
\hline & Denmark & $\begin{array}{l}\text { Faroe } \\
\text { Islands }\end{array}$ & Greenland & Finland & Åland & Iceland & Norway & Sweden \\
\hline \multicolumn{9}{|l|}{$\mathrm{C} 10$} \\
\hline \multicolumn{9}{|c|}{ Lipid modifying agents } \\
\hline 2005 & 47.2 & 42.8 & 55.1 & 55.7 & 23.9 & 48.1 & 67.9 & 44.3 \\
\hline 2010 & 108.4 & 80.4 & 52.3 & 98.8 & 43.3 & 71.5 & 112.7 & 75.6 \\
\hline 2013 & 125.7 & 112.1 & 55.4 & 101.9 & 47.1 & 90.5 & 120.0 & 85.9 \\
\hline 2014 & 132.4 & 127.7 & 51.7 & 102.6 & 50.1 & 88.7 & 122.4 & 92.0 \\
\hline 2015 & 141.3 & 134.0 & 58.7 & 105.4 & 49.4 & 89.7 & 127.9 & 99.3 \\
\hline \multicolumn{9}{|c|}{ C10AA } \\
\hline \multicolumn{9}{|c|}{$\begin{array}{l}\text { HMG CoA reductase } \\
\text { inhibitors (statins) }\end{array}$} \\
\hline 2005 & 46.5 & 42.3 & 55.0 & 53.9 & 23.1 & 87.3 & 67.2 & 42.0 \\
\hline 2010 & 105.9 & 78.7 & 52.2 & 95.5 & 41.8 & 70.2 & 109.9 & 72.5 \\
\hline 2013 & 122.8 & 110.7 & 55.1 & 98.3 & 45.7 & 89.2 & 115.7 & 82.8 \\
\hline 2014 & 129.4 & 126.1 & 51.6 & 98.8 & 48.7 & 87.6 & 118.1 & 88.8 \\
\hline 2015 & 138.2 & 132.0 & 58.6 & 101.3 & 47.6 & 88.5 & 122.2 & 95.9 \\
\hline \multicolumn{9}{|c|}{$\begin{array}{l}\text { C10AX } \\
\text { Other lipid modifying } \\
\text { agents }\end{array}$} \\
\hline 2005 & 0.1 & 0.1 & - & 1.0 & 0.3 & 0.5 & 0.6 & 0.9 \\
\hline 2010 & 1.5 & 1.0 & - & 2.5 & 1.2 & 0.5 & 2.7 & 2.1 \\
\hline 2013 & 2.0 & 1.0 & 0.2 & 3.0 & 1.2 & 0.5 & 3.8 & 2.2 \\
\hline 2014 & 2.1 & 1.2 & 0.1 & 3.2 & 1.2 & 0.4 & 4.1 & 2.3 \\
\hline 2015 & 2.2 & 1.6 & 0.2 & 3.5 & 1.5 & 0.6 & 4.5 & 2.5 \\
\hline
\end{tabular}


Morbidity, Medical Treatment, Accidents and Pharmaceutical products

Table 3.7.11 Proportion of the population per 1000 by age and gender (oneyear prevalence) receiving at least one serum lipid modifying agent (ATC-group C10), 2015

\begin{tabular}{|c|c|c|c|}
\hline & Men & Women & Total \\
\hline \multicolumn{4}{|c|}{ Denmark } \\
\hline $0-14$ & - & - & - \\
\hline $15-24$ & 1 & 1 & 1 \\
\hline $25-44$ & 18 & 11 & 14 \\
\hline $45-64$ & 165 & 128 & 147 \\
\hline $65-74$ & 384 & 330 & 357 \\
\hline $75+$ & 442 & 362 & 395 \\
\hline Total & .. & .. & .. \\
\hline \multicolumn{4}{|c|}{ Faroe Islands } \\
\hline $0-14$ & - & - & - \\
\hline $15-24$ & 1 & 1 & 1 \\
\hline $25-44$ & 21 & 12 & 17 \\
\hline $45-64$ & 178 & 132 & 156 \\
\hline $65-74$ & 431 & 360 & 397 \\
\hline $75+$ & 532 & 370 & 439 \\
\hline Total & 124 & 102 & 114 \\
\hline \multicolumn{4}{|c|}{ Finland } \\
\hline $0-14$ & - & - & .. \\
\hline $15-24$ & 1 & 1 & .. \\
\hline $25-44$ & 17 & 7 & .. \\
\hline $45-64$ & 180 & 117 & .. \\
\hline $65-74$ & 395 & 331 & .. \\
\hline $75+$ & 475 & 397 & .. \\
\hline Total & .. & .. & .. \\
\hline \multicolumn{4}{|c|}{ Iceland } \\
\hline $0-14$ & - & - & - \\
\hline $15-24$ & 1 & 1 & 1 \\
\hline $25-44$ & 12 & 6 & 9 \\
\hline $45-64$ & 174 & 102 & 138 \\
\hline $65-74$ & 410 & 312 & 382 \\
\hline $75+$ & 505 & 345 & 415 \\
\hline Total & .. & .. & .. \\
\hline \multicolumn{4}{|c|}{ Norway } \\
\hline $0-14$ & - & - & 0 \\
\hline $15-24$ & 2 & 2 & 2 \\
\hline $25-44$ & 18 & 8 & 13 \\
\hline $45-64$ & 165 & 113 & 139 \\
\hline $65-74$ & 395 & 331 & 363 \\
\hline $75+$ & 442 & 346 & 385 \\
\hline Total & 111 & 93 & 102 \\
\hline \multicolumn{4}{|c|}{ Sweden } \\
\hline $0-14$ & - & - & - \\
\hline $15-24$ & 1 & 1 & 1 \\
\hline $25-44$ & 11 & 5 & 8 \\
\hline $45-64$ & 127 & 81 & 104 \\
\hline $65-74$ & 352 & 263 & 306 \\
\hline $75+$ & 430 & 301 & 354 \\
\hline Total &.. & .. & .. \\
\hline
\end{tabular}


Table 3.7.12 Proportion of the population per 1000 women) by age $15-49$ (one year prevalence) receiving at least one type of hormonal contraceptive (ATC-groups G03A and G02BB), 20151

\begin{tabular}{lccccc}
\hline Age & Denmark & $\begin{array}{c}\text { Faroe } \\
\text { Islands }\end{array}$ & Iceland & Norway & Sweden \\
\cline { 2 - 6 } $15-19$ & 511 & 348 & 477 & 417 & 324 \\
$20-24$ & 587 & 604 & 594 & 602 & 410 \\
$25-29$ & 426 & 348 & 420 & 433 & 319 \\
$30-34$ & 282 & 221 & 276 & 268 & 204 \\
$35-39$ & 200 & 172 & 203 & 177 & 157 \\
$40-44$ & 138 & 145 & 146 & 117 & 121 \\
$45-49$ & 79 & 98 & 93 & 68 & 83 \\
\hline
\end{tabular}

1 Excl. Implants (G03AC08), injections (G03AC06) and emergency contraceptives (G03AD). The group does not contain injections or intrauterine devices

Figure 3.7.4 Proportion of women/1 000 between 15 and 49 (one year prevalence) receiving at least one type of hormonal contraceptive and intra-vaginal contraceptive (ATC-groups G03A and G02BB), 2005-2015

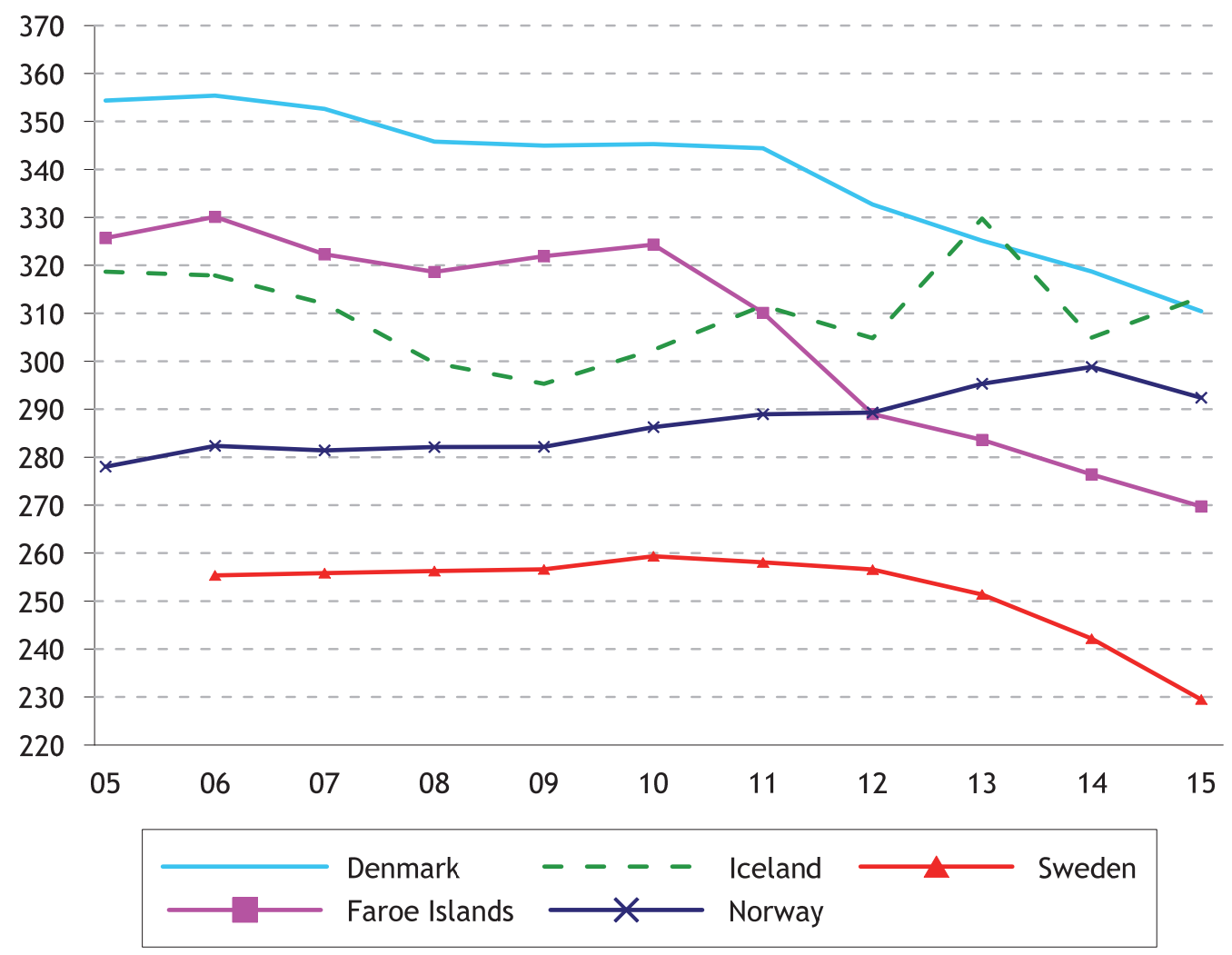


Table 3.7.13 Sales of estrogens (ATC group G03C) and progestogens and estrogens in combination (ATC-group G03F), systemic use, DDD/1 000 women/day, 2005-2015

\begin{tabular}{lcccccccc}
\hline & Denmark & $\begin{array}{c}\text { Faroe } \\
\text { Islands }\end{array}$ & Greenland & Finland & Åland & Iceland & Norway Sweden \\
\hline 2005 & 27.8 & 29.4 &.. & 66.2 & 51.3 & 64.9 & 35.9 & 33.0 \\
2010 & 17.6 & 20.8 & 6.0 & 50.8 & 38.0 & 50.1 & 20.7 & 18.1 \\
2013 & 14.5 & 17.7 & 3.9 & 36.6 & 39.0 & 44.6 & 20.4 & 15.9 \\
2014 & 14.2 & 19.0 & 4.1 & 42.6 & 38.9 & 42.9 & 21.1 & 15.4 \\
2015 & 13.7 & 18.7 & 3.7 & 33.6 & 35.3 & 42.8 & 20.8 & 14.7 \\
\hline
\end{tabular}

Table 3.7.14 Sales of estrogens (ATC-group G03C), vaginal administration, DDD/1 000 women/day 2005-20151

\begin{tabular}{rrrrrrrrr}
\hline & Denmark & $\begin{array}{c}\text { Faroe } \\
\text { lslands }\end{array}$ & Greenland & Finland & Åland & Iceland & Norway & Sweden \\
\hline 2005 & 8.8 & 9.2 &.. & 21.4 & 15.6 & 43.5 & 13.4 & 19.9 \\
2010 & 11.4 & 10.9 & 2.6 & 29.3 & 29.4 & 35.2 & 13.2 & 20.7 \\
2013 & 12.2 & 13.4 & 3.4 & 20.1 & 26.3 & 31.5 & 9.0 & 18.6 \\
2014 & 9.5 & 9.5 & 2.9 & 19.7 & 28.6 & 30.3 & 8.7 & 22.0 \\
2015 & 9.0 & 8.6 & 1.8 & 26.4 & 29.9 & 30.0 & 8.9 & 23.5 \\
\hline
\end{tabular}

1 Vaginal tablets, vaginal gel and vaginal insert

Table 3.7.15 Sales of drugs for urinary frequency and incontinence (ATC-group G04BD), DDD/1 000 inhabitants/day, 2005-2015

\begin{tabular}{lcccccccc}
\hline & Denmark & $\begin{array}{c}\text { Faroe } \\
\text { Islands }\end{array}$ & Greenland & Finland & Åland & Iceland & Norway & Sweden \\
\hline 2005 & 3.0 & 2.1 &.. & 3.2 & 3.1 & 3.2 & 4.7 & 3.9 \\
2010 & 5.0 & 4.2 & 0.6 & 4.5 & 3.3 & 7.0 & 7.8 & 5.0 \\
2013 & 5.8 & 5.0 & 0.5 & 5.3 & 4.8 & 7.8 & 8.9 & 5.4 \\
2014 & 5.8 & 5.1 & 0.5 & 5.5 & 4.9 & 8.0 & 9.4 & 5.7 \\
2015 & 6.0 & 5.2 & 0.6 & 5.9 & 5.1 & 8.3 & 9.6 & 5.9 \\
\hline
\end{tabular}

Table 3.7.16 Sales of drugs used in erectile dysfunction (ATC-group G04BE), DDD/1 000 men/day, 2005-2015

\begin{tabular}{lcccccccc}
\hline & Denmark & $\begin{array}{c}\text { Faroe } \\
\text { Islands }\end{array}$ & Greenland & Finland & Åland & Iceland & Norway & Sweden \\
\hline 2005 & 2.2 & 1.2 & 0.7 & 4.0 & 1.4 & 3.0 & 2.6 & 2.4 \\
2010 & 3.1 & 1.3 & 1.3 & 6.1 & 2.2 & 2.6 & 3.5 & 2.9 \\
2013 & 4.1 & 1.9 & 2.3 & 11.1 & 4.0 & 4.0 & 4.3 & 3.2 \\
2014 & 5.9 & 2.5 & 2.9 & 12.2 & 4.6 & 4.3 & 4.6 & 3.9 \\
2015 & 6.8 & 3.0 & 3.0 & 13.5 & 5.2 & 5.4 & 5.0 & 4.3 \\
\hline
\end{tabular}


Morbidity, Medical Treatment, Accidents and Pharmaceutical products

Table 3.7.17 Sales of systemic hormonal prep, excl. sex hormones (ATC-group H), DDD/1 000 inhabitants/day

\begin{tabular}{lrrrrrrrr}
\hline & Denmark & $\begin{array}{c}\text { Faroe } \\
\text { Islands }\end{array}$ & Greenland & Finland & Áland & Iceland & Norway & Sweden \\
\hline $\begin{array}{l}\text { H02A } \\
\text { Corticosteroids for } \\
\text { system use, plain }\end{array}$ & & & & & & & & \\
2005 & 13.4 & 10.4 &.. & 16.4 & 19.6 & 10.6 & 15.4 & 14.3 \\
2010 & 13.8 & 11.0 & 7.3 & 18.6 & 19.5 & 11.6 & 17.1 & 15.4 \\
2013 & 13.7 & 11.6 & 7.0 & 18.8 & 19.0 & 12.6 & 17.5 & 15.7 \\
2014 & 13.9 & 12.2 & 7.9 & 19.4 & 20.2 & 14.5 & 18.1 & 16.0 \\
2015 & 13.7 & 11.3 & 8.2 & 18.7 & 19.9 & 14.7 & 18.5 & 16.0 \\
H03A & & & & & & & & \\
$\begin{array}{l}\text { Thyroid } \\
\text { preparations }\end{array}$ & & & & & & & & \\
2005 & 10.1 & 11.4 &.. & 19.7 & 25.5 & 18.2 & 20.8 & 22.8 \\
2010 & 13.4 & 13.7 & 2.4 & 27.6 & 30.3 & 22.3 & 24.0 & 24.7 \\
2013 & 15.6 & 14.7 & 3.6 & 30.9 & 32.5 & 26.8 & 24.8 & 25.5 \\
2014 & 16.3 & 15.1 & 3.1 & 32.6 & 33.4 & 29.0 & 24.7 & 25.8 \\
2015 & 17.0 & 15.5 & 3.4 & 34.0 & 34.5 & 29.3 & 24.3 & 26.0 \\
\hline
\end{tabular}


Table 3.7.18 Sales of antibacterials for systemic use (ATC-group J01), DDD/1 000 inhabitants/day, 2005-2015

\begin{tabular}{|c|c|c|c|c|c|c|c|c|}
\hline & Denmark & $\begin{array}{l}\text { Faroe } \\
\text { Islands }\end{array}$ & Greenland & Finland & Åland & Iceland & Norway & Sweden \\
\hline \multicolumn{9}{|c|}{$\begin{array}{l}\text { J01 } \\
\text { Antibacterials for } \\
\text { systemic use }\end{array}$} \\
\hline 2005 & 16.5 & 18.0 & 20.4 & 21.6 & 22.1 & 23.0 & 18.1 & 16.5 \\
\hline 2010 & 18.8 & 17.3 & 17.3 & 21.8 & 19.5 & 22.2 & 19.6 & 15.7 \\
\hline 2013 & 18.7 & 17.0 & 17.8 & 20.9 & 17.6 & 21.7 & 19.6 & 14.7 \\
\hline 2014 & 18.2 & 16.5 & 16.0 & 20.7 & 17.4 & 21.5 & 19.2 & 14.1 \\
\hline 2015 & 18.2 & 17.2 & 20.2 & 19.8 & 17.0 & 22.0 & 18.7 & 13.9 \\
\hline \multicolumn{9}{|c|}{$\begin{array}{l}\text { J01A } \\
\text { Tetracyclines }\end{array}$} \\
\hline 2005 & 1.3 & 1.2 & 3.1 & 4.2 & 3.4 & 5.4 & 3.1 & 3.5 \\
\hline 2010 & 1.7 & 1.5 & 1.1 & 4.3 & 3.6 & 5.1 & 3.1 & 3.3 \\
\hline 2013 & 2.0 & 1.8 & 0.9 & 4.5 & 4.0 & 4.7 & 3.5 & 3.1 \\
\hline 2014 & 1.7 & 1.7 & 0.9 & 4.3 & 3.8 & 4.5 & 3.4 & 2.9 \\
\hline 2015 & 1.6 & 1.5 & 1.0 & 4.1 & 3.5 & 4.8 & 3.3 & 2.7 \\
\hline \multicolumn{9}{|c|}{$\begin{array}{l}\text { J01C } \\
\text { Beta-lactam } \\
\text { antibacterials, } \\
\text { penicillins }\end{array}$} \\
\hline 2005 & 10.1 & 11.5 & 11.8 & 6.3 & 7.9 & 11.8 & 7.6 & 7.3 \\
\hline 2010 & 11.4 & 10.7 & 11.4 & 7.2 & 7.2 & 12.0 & 8.5 & 7.9 \\
\hline 2013 & 11.7 & 10.1 & 12.5 & 6.8 & 7.6 & 11.6 & 8.2 & 7.5 \\
\hline 2014 & 11.7 & 9.4 & 10.4 & 7.0 & 7.6 & 11.4 & 8.1 & 7.2 \\
\hline 2015 & 11.9 & 10.2 & 14.3 & 7.0 & 8.4 & 11.5 & 7.9 & 7.2 \\
\hline \multicolumn{9}{|c|}{$\begin{array}{l}\text { J01CA } \\
\text { Penicillins with } \\
\text { extended spectrum }\end{array}$} \\
\hline 2005 & 3.2 & 3.0 & 4.0 & 3.4 & 5.0 & 4.3 & 2.5 & 1.6 \\
\hline 2010 & 3.8 & 2.9 & 3.9 & 4.1 & 4.5 & 4.2 & 3.2 & 1.7 \\
\hline 2013 & 3.8 & 1.9 & 4.2 & 3.9 & 4.5 & 3.9 & 3.3 & 1.6 \\
\hline 2014 & 3.9 & 1.8 & 3.8 & 4.0 & 4.4 & 3.5 & 3.3 & 1.6 \\
\hline 2015 & 4.0 & 1.9 & 5.4 & 4.0 & 5.0 & 3.7 & 3.1 & 1.6 \\
\hline \multicolumn{9}{|c|}{$\begin{array}{l}\text { J01CE } \\
\text { Beta-lactamase } \\
\text { sensitive penicill }\end{array}$} \\
\hline 2005 & 5.7 & 7.2 & 6.9 & 1.7 & 2.2 & 3.0 & 4.5 & 4.1 \\
\hline 2010 & 5.5 & 6.4 & 5.8 & 1.6 & 1.9 & 2.5 & 4.4 & 4.2 \\
\hline 2013 & 4.9 & 6.2 & 5.5 & 1.4 & 1.6 & 2.1 & 4.1 & 3.7 \\
\hline 2014 & 4.6 & 5.6 & 4.0 & 1.4 & 1.6 & 2.2 & 3.8 & 3.4 \\
\hline 2015 & 4.5 & 6.0 & 5.2 & 1.4 & 1.5 & 2.0 & 3.8 & 3.4 \\
\hline \multicolumn{9}{|c|}{$\begin{array}{l}\text { J01CF } \\
\text { Beta-lactamase } \\
\text { resistant penicillins }\end{array}$} \\
\hline 2005 & 1.2 & 1.2 & 0.9 & 0.1 & 0.4 & 1.4 & 0.5 & 1.4 \\
\hline 2010 & 1.3 & 1.2 & 1.4 & 0.0 & 0.0 & 1.3 & 0.8 & 1.7 \\
\hline 2013 & 1.5 & 1.3 & 1.7 & 0.1 & 0.7 & 1.2 & 0.8 & 1.9 \\
\hline 2014 & 1.6 & 1.4 & 1.5 & 0.1 & 0.8 & 1.2 & 0.8 & 1.9 \\
\hline 2015 & 1.6 & 1.4 & 2.2 & 0.1 & 1.1 & 1.1 & 0.9 & 1.9 \\
\hline
\end{tabular}

The table continues 
Morbidity, Medical Treatment, Accidents and Pharmaceutical products

Table 3.7.18 Sales of antibacterials for systemic use (ATC-group J01), DDD/1 000 inhabitants/day, 2005-2015, continued

\begin{tabular}{|c|c|c|c|c|c|c|c|c|}
\hline & Denmark & $\begin{array}{l}\text { Faroe } \\
\text { Islands }\end{array}$ & Greenland & Finland & Åland & Iceland & Norway & Sweden \\
\hline \multicolumn{9}{|c|}{$\begin{array}{l}\text { J01CR } \\
\text { Combinations of } \\
\text { penicilins incl. beta- } \\
\text { lactamase inhibitors }\end{array}$} \\
\hline 2005 & 0.1 & 0.1 & - & 1.1 & 0.4 & 3.2 & - & 0.2 \\
\hline 2010 & 0.8 & 0.2 & 0.3 & 1.5 & 0.8 & 4.0 & 0.0 & 0.3 \\
\hline 2013 & 1.5 & 0.6 & 1.0 & 1.4 & 0.9 & 4.3 & 0.0 & 0.3 \\
\hline 2014 & 1.6 & 0.7 & 1.0 & 1.5 & 0.8 & 4.5 & 0.1 & 0.4 \\
\hline 2015 & 1.8 & 0.9 & 1.5 & 1.5 & 0.7 & 4.7 & 0.1 & 0.4 \\
\hline \multicolumn{9}{|c|}{$\begin{array}{l}\text { J01D } \\
\text { Other betalactam } \\
\text { anti-bacterials and } \\
\text { cephalosporins }\end{array}$} \\
\hline 2005 & 0.3 & 0.5 & 1.0 & 3.1 & 1.7 & 0.5 & 0.6 & 0.7 \\
\hline 2010 & 0.4 & 0.4 & 0.4 & 3.2 & 1.7 & 0.6 & 0.5 & 0.4 \\
\hline 2013 & 0.4 & 0.6 & 0.3 & 3.2 & 1.2 & 0.8 & 0.5 & 0.3 \\
\hline 2014 & 0.4 & 0.6 & 0.4 & 3.2 & 1.1 & 0.8 & 0.5 & 0.3 \\
\hline 2015 & 0.4 & 0.7 & 0.4 & 3.0 & 1.0 & 0.8 & 0.4 & 0.3 \\
\hline \multicolumn{9}{|c|}{$\begin{array}{l}\text { J01E } \\
\text { Sulphonamides and } \\
\text { Trimethoprim }\end{array}$} \\
\hline 2005 & 0.9 & 1.0 & 0.6 & 1.9 & 1.0 & 1.9 & 1.1 & 0.9 \\
\hline 2010 & 0.8 & 1.2 & 0.5 & 1.6 & 0.8 & 0.9 & 0.9 & 0.6 \\
\hline 2013 & 0.9 & 1.5 & 0.6 & 1.4 & 0.8 & 0.8 & 0.8 & 0.5 \\
\hline 2014 & 0.9 & 1.7 & 0.6 & 1.3 & 0.7 & 0.8 & 0.8 & 0.5 \\
\hline 2015 & 0.8 & 1.5 & 0.6 & 1.3 & 0.7 & 0.7 & 0.8 & 0.5 \\
\hline \multicolumn{9}{|c|}{$\begin{array}{l}\text { J01F } \\
\text { Macrolides, } \\
\text { lincosamides } \\
\text { and streptogramins }\end{array}$} \\
\hline 2005 & 2.5 & 2.1 & 3.6 & 2.1 & 1.1 & 1.8 & 2.1 & 0.8 \\
\hline 2010 & 2.6 & 1.7 & 2.7 & 1.6 & 1.1 & 1.6 & 2.0 & 0.7 \\
\hline 2013 & 2.1 & 1.5 & 2.4 & 1.4 & 0.8 & 1.7 & 1.9 & 0.7 \\
\hline 2014 & 1.9 & 1.5 & 2.5 & 1.3 & 0.7 & 1.7 & 1.7 & 0.6 \\
\hline 2015 & 1.9 & 1.7 & 2.6 & 1.2 & 0.7 & 1.9 & 1.5 & 0.6 \\
\hline \multicolumn{9}{|c|}{$\begin{array}{l}\text { J01M } \\
\text { Quinolone } \\
\text { anti-bacterials }\end{array}$} \\
\hline 2005 & 0.5 & 0.3 & 0.2 & 1.3 & 1.1 & 0.8 & 0.6 & 1.2 \\
\hline 2010 & 0.8 & 0.5 & 0.5 & 1.2 & 1.1 & 1.0 & 0.7 & 0.9 \\
\hline 2013 & 0.7 & 0.6 & 0.5 & 1.1 & 0.7 & 1.1 & 0.7 & 0.9 \\
\hline 2014 & 0.7 & 0.6 & 0.6 & 1.2 & 0.9 & 1.0 & 0.6 & 0.9 \\
\hline 2015 & 0.7 & 0.6 & 0.4 & 1.2 & 0.8 & 1.1 & 0.6 & 0.8 \\
\hline \multicolumn{9}{|c|}{$\begin{array}{l}\text { J01X } \\
\text { Other } \\
\text { Anti-bacterials }\end{array}$} \\
\hline 2005 & 0.9 & 1.3 & 0.8 & 2.8 & 5.9 & 0.4 & 3.0 & 2.2 \\
\hline 2010 & 0.9 & 1.1 & 0.5 & 2.7 & 4.1 & 1.0 & 3.8 & 1.7 \\
\hline 2013 & 0.9 & 0.9 & 0.6 & 2.4 & 2.5 & 1.1 & 4.1 & 1.7 \\
\hline 2014 & 0.9 & 1.1 & 0.7 & 2.3 & 2.5 & 1.2 & 4.1 & 1.7 \\
\hline 2015 & 0.9 & 1.1 & 0.8 & 2.1 & 1.8 & 1.2 & 4.2 & 1.7 \\
\hline
\end{tabular}


Figure 3.7.5 Sales of antibacterials for systemic use (ATC-group J01), DDD/1 000 inhabitants/day, 2005-2015

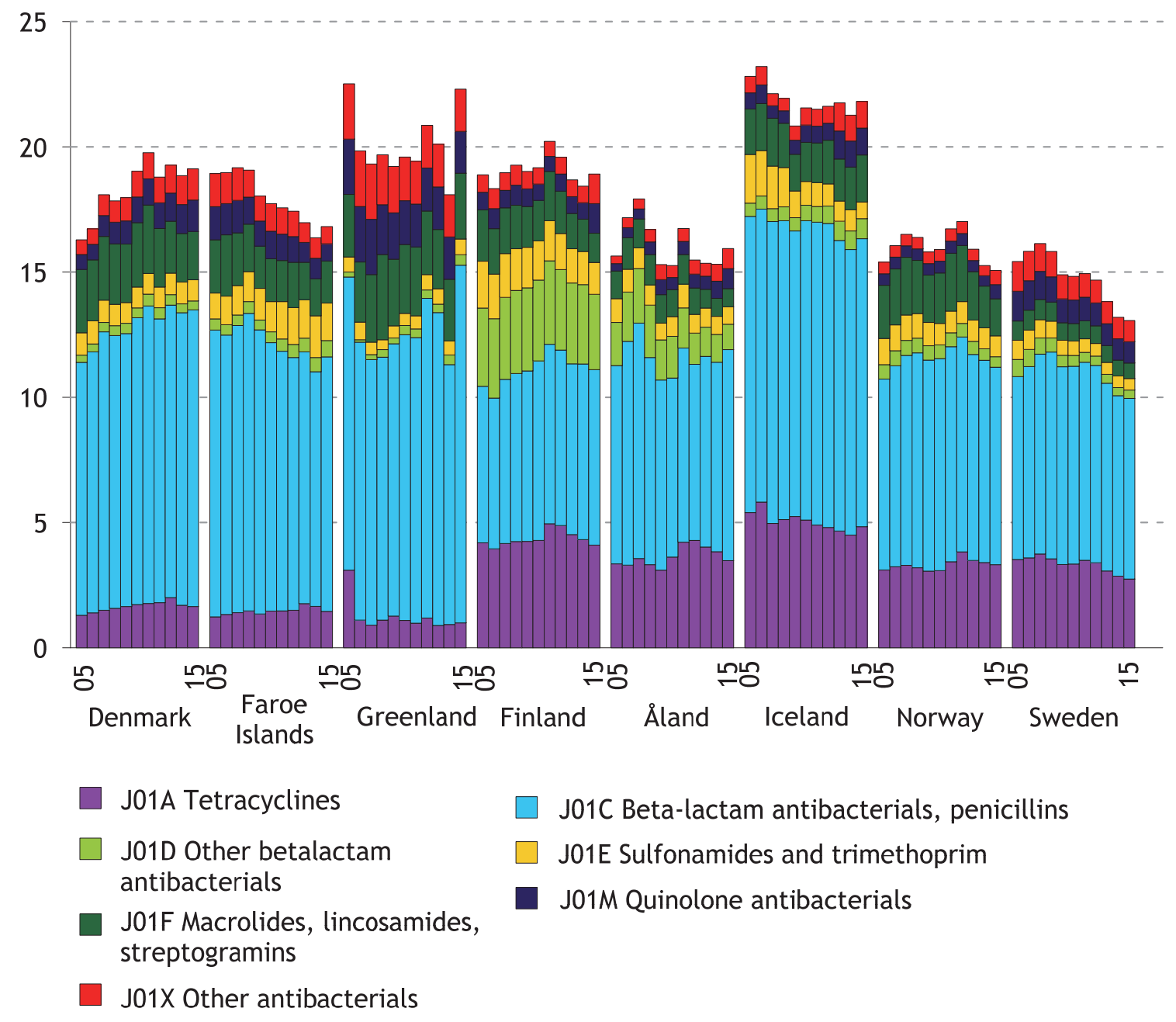


Figure 3.7.6 Sales of penicillins (ATC-group J01C), DDD/1 000 inhabitants/day, 2005-2015

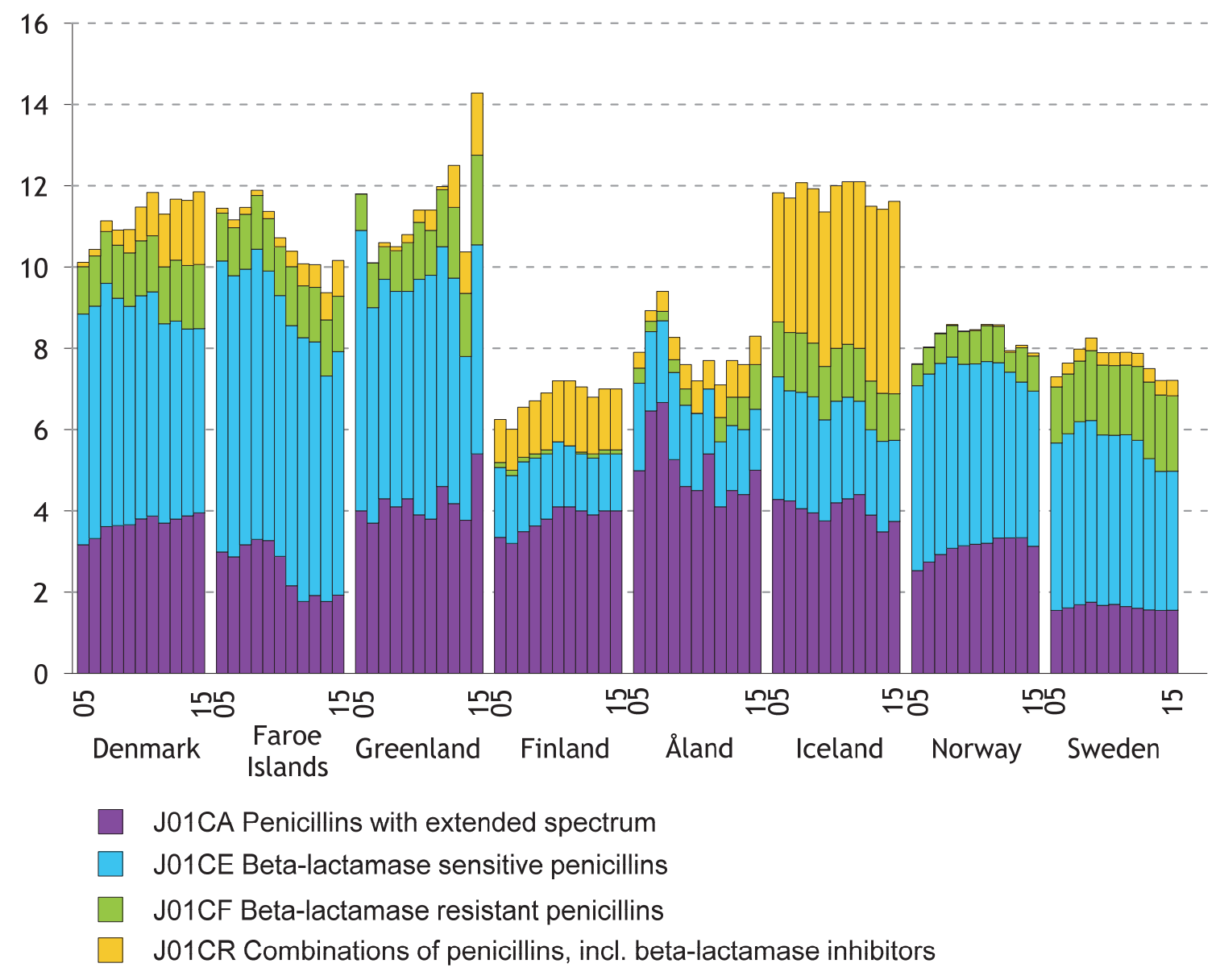


Morbidity, Medical Treatment, Accidents and Pharmaceutical products

Table 3.7.19 Proportion of the population per 1000 by age and gender (oneyear prevalence) receiving at least one penicillin (ATC-group J01C), 2015

\begin{tabular}{|c|c|c|c|}
\hline & Men & Women & Total \\
\hline \multicolumn{4}{|c|}{ Denmark } \\
\hline $0-14$ & 179 & 183 & 181 \\
\hline $15-24$ & 121 & 238 & 178 \\
\hline $25-44$ & 147 & 253 & 200 \\
\hline $45-64$ & 182 & 244 & 213 \\
\hline $65-74$ & 247 & 280 & 264 \\
\hline $75+$ & 341 & 371 & 358 \\
\hline Total & .. & .. & .. \\
\hline \multicolumn{4}{|c|}{ Faroe Islands } \\
\hline $0-14$ & 193 & 194 & 194 \\
\hline $15-24$ & 154 & 222 & 186 \\
\hline $25-44$ & 182 & 255 & 216 \\
\hline $45-64$ & 192 & 233 & 212 \\
\hline $65-74$ & 251 & 289 & 269 \\
\hline $75+$ & 336 & 303 & 317 \\
\hline Total & 199 & 240 & 218 \\
\hline \multicolumn{4}{|c|}{ Finland } \\
\hline $0-14$ & 219 & 205 & .. \\
\hline $15-24$ & 111 & 203 & .. \\
\hline $25-44$ & 123 & 198 & .. \\
\hline $45-64$ & 124 & 186 & .. \\
\hline $65-74$ & 126 & 179 & .. \\
\hline $75+$ & 151 & 232 & .. \\
\hline Total & .. & .. & .. \\
\hline \multicolumn{4}{|c|}{ Iceland } \\
\hline $0-14$ & 280 & 285 & 282 \\
\hline $15-24$ & 289 & 336 & 262 \\
\hline $25-44$ & 205 & 343 & 273 \\
\hline $45-64$ & 251 & 374 & 313 \\
\hline $65-74$ & 292 & 431 & 372 \\
\hline $75+$ & 189 & 377 & 346 \\
\hline Total & .. & .. & .. \\
\hline \multicolumn{4}{|c|}{ Norway } \\
\hline $0-14$ & 110 & 111 & 111 \\
\hline $15-24$ & 89 & 186 & 136 \\
\hline $25-44$ & 97 & 185 & 140 \\
\hline $45-64$ & 116 & 179 & 147 \\
\hline $65-74$ & 163 & 218 & 191 \\
\hline $75+$ & 214 & 254 & 238 \\
\hline Total & 119 & 186 & 152 \\
\hline \multicolumn{4}{|c|}{ Sweden } \\
\hline $0-14$ & 145 & 140 & 143 \\
\hline $15-24$ & 78 & 146 & 111 \\
\hline $25-44$ & 83 & 150 & 116 \\
\hline $45-64$ & 97 & 148 & 122 \\
\hline $65-74$ & 133 & 179 & 156 \\
\hline $75+$ & 178 & 218 & 201 \\
\hline Total & .. & .. & .. \\
\hline
\end{tabular}


Morbidity, Medical Treatment, Accidents and Pharmaceutical products

Table 3.7.20 Sales of antimycotics for systemic use (ATC group J02A), DDD/1 000 inhabitants/day, 2005-2015

\begin{tabular}{lcccccccc}
\hline & Denmark & $\begin{array}{c}\text { Faroe } \\
\text { Islands }\end{array}$ & $\begin{array}{c}\text { Green- } \\
\text { land }\end{array}$ & Finland & Åland & Iceland & Norway & Sweden \\
\hline & & & & & & & & \\
2005 & 0.5 & 0.5 & 0.3 & 0.4 & 0.4 & 0.5 & 0.2 & 0.2 \\
2010 & 0.7 & 0.5 & 0.0 & 0.4 & 0.3 & 0.7 & 0.2 & 0.3 \\
2013 & 0.7 & 0.5 & 0.3 & 0.5 & 0.4 & 0.5 & 0.3 & 0.3 \\
2014 & 0.7 & 0.5 & 0.3 & 0.5 & 0.4 & 0.5 & 0.2 & 0.3 \\
2015 & 0.7 & 0.5 & 0.3 & 0.5 & 0.4 & 0.6 & 0.3 & 0.3 \\
\hline
\end{tabular}

Table 3.7.21 Sales of antivirals for systemic use (ATC group J05), DDD/1 000 inhabitants/day, 2005-2015

\begin{tabular}{lcccccccc}
\hline & Denmark & $\begin{array}{c}\text { Faroe } \\
\text { Islands }\end{array}$ & $\begin{array}{c}\text { Green- } \\
\text { land }\end{array}$ & Finland & Åland & Iceland & Norway & Sweden \\
\hline & & & & & & & & \\
2005 & 1.3 & 0.2 & 1.9 & 0.7 & 0.3 & 0.9 & 0.9 & 1.1 \\
2010 & 1.7 & 0.4 & 1.6 & 0.9 & 0.3 & 0.9 & 1.1 & 1.4 \\
2013 & 2.0 & 0.6 & 1.5 & 1.1 & 0.4 & 1.1 & 1.4 & 1.6 \\
2014 & 2.1 & 0.5 & 1.4 & 1.2 & 0.5 & 1.0 & 1.5 & 1.7 \\
2015 & 2.3 & 0.5 & 1.7 & 1.2 & 0.7 & 1.1 & 1.5 & 1.8 \\
\hline
\end{tabular}

Table 3.7.22 Sales of antineoplastic agents (ATC-group L01), Euro per $1000 /$ inhabitants at 2015 prices, 2005-2015

\begin{tabular}{lrrrrrrrr}
\hline & Denmark & $\begin{array}{l}\text { Faroe } \\
\text { Islands }\end{array}$ & Greenland & Finland & Åland & Iceland & Norway & Sweden \\
\hline & & & & & & & & \\
2005 & 21677 & 8061 & - & 16894 & 30247 & - & 14371 & 15488 \\
2010 & 41494 & 13055 & 13171 & 29208 & 38734 & 62795 & 22688 & 26129 \\
2013 & 43582 & 19447 & 12133 & 31782 & 37465 & 96129 & 25237 & 27649 \\
2014 & 48664 & 19465 & 14417 & 32820 & 41357 & 100833 & 28350 & 28899 \\
2015 & 50743 & 19176 & 17262 & 35051 & 41609 & 107343 & 32347 & 32009 \\
\hline
\end{tabular}


Table 3.7.23 Sales of immunomodulating agents (ATC-group L02, L03, L04), DDD/1 000 inhabitants/day, 2005-2015

\begin{tabular}{|c|c|c|c|c|c|c|c|c|}
\hline & Denmark & $\begin{array}{l}\text { Faroe } \\
\text { Islands }\end{array}$ & Greenland & Finland & Åland & Iceland & Norway & Sweden \\
\hline \multicolumn{9}{|l|}{ L02 } \\
\hline \multicolumn{9}{|c|}{ Endocrine therapy } \\
\hline 2005 & 4.3 & 2.5 & & 4.7 & 6.1 & 4.7 & 5.0 & 5.8 \\
\hline 2010 & 6.0 & 3.7 & 0.9 & 6.3 & 6.8 & 5.4 & 5.9 & 7.1 \\
\hline 2013 & 6.7 & 5.5 & 1.0 & 7.0 & 6.1 & 5.9 & 6.2 & 7.9 \\
\hline 2014 & 6.8 & 5.2 & 1.7 & 7.1 & 5.6 & 5.0 & 6.0 & 8.3 \\
\hline 2015 & 7.5 & 5.7 & 1.5 & 7.2 & 5.7 & 5.8 & 6.0 & 8.8 \\
\hline \multirow{2}{*}{\multicolumn{9}{|c|}{$\begin{array}{l}\text { L03 } \\
\text { Immunostimulants }\end{array}$}} \\
\hline & & & & & & & & \\
\hline 2005 & 0.8 & 0.3 & & 0.9 & 0.5 & 1.1 & 0.8 & 1.0 \\
\hline 2010 & 1.3 & 1.0 & 0.1 & 1.4 & 0.4 & 0.7 & 1.0 & 1.0 \\
\hline 2013 & 1.3 & 0.8 & 0.1 & 1.2 & 0.5 & 0.8 & 0.9 & 0.8 \\
\hline 2014 & 1.1 & 0.7 & 0.2 & 1.1 & 0.6 & 0.7 & 0.7 & 0.7 \\
\hline 2015 & 0.9 & 0.8 & 0.1 & 1.1 & 0.6 & 0.5 & 0.6 & 0.5 \\
\hline \multicolumn{9}{|c|}{$\begin{array}{l}\text { L04 } \\
\text { Immunosuppressants }\end{array}$} \\
\hline 2005 & 4.4 & 4.0 & & 5.2 & 7.5 & 4.5 & 5.1 & 4.8 \\
\hline 2010 & 7.2 & 7.3 & $4 . \ddot{5}$ & 7.8 & 9.3 & 4.8 & 8.0 & 7.8 \\
\hline 2013 & 8.6 & 9.1 & 6.4 & 8.9 & 9.8 & 9.5 & 9.8 & 9.0 \\
\hline 2014 & 9.1 & 9.8 & 6.8 & 9.8 & 9.7 & 10.2 & 10.6 & 9.4 \\
\hline 2015 & 9.8 & 10.5 & 7.5 & 10.3 & 10.7 & 10.8 & 11.3 & 9.9 \\
\hline \multirow{2}{*}{\multicolumn{9}{|c|}{$\begin{array}{l}\text { L04AB } \\
\text { Tumour necrosis factor } \\
\text { alpha (TNF-a) inhibitors }\end{array}$}} \\
\hline & & & & & & & & \\
\hline 2005 & 0.6 & 0.7 & 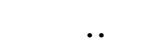 & 0.6 & 2.5 & 0.7 & 1.3 & 0.9 \\
\hline 2010 & 1.8 & 2.4 & 1.5 & 1.4 & 3.5 & 0.7 & 2.5 & 1.9 \\
\hline 2013 & 2.3 & 3.4 & 2.4 & 2.0 & 4.0 & 3.5 & 3.3 & 2.4 \\
\hline 2014 & 2.5 & 4.0 & 2.7 & 2.2 & 3.6 & 3.9 & 3.7 & 2.6 \\
\hline 2015 & 2.7 & 4.7 & 3.1 & 2.4 & 4.1 & 4.2 & 4.1 & 2.8 \\
\hline
\end{tabular}


Morbidity, Medical Treatment, Accidents and Pharmaceutical products

Table 3.7.24 Sales of analgesics, including anti-inflammatory agents (ATC-groups M01A, N02A and N02B), DDD/1 000 inhabitants/day, 2005-2015

\begin{tabular}{|c|c|c|c|c|c|c|c|c|}
\hline & Denmark & $\begin{array}{l}\text { Faroe } \\
\text { Islands }\end{array}$ & Greenland ${ }^{1}$ & Finland & Åland & Iceland & Norway $^{2}$ & Sweden $^{2}$ \\
\hline \multicolumn{9}{|l|}{ M01A } \\
\hline \multicolumn{9}{|c|}{$\begin{array}{l}\text { Anti-inflammatory } \\
\text { and antirheumatic } \\
\text { products, } \\
\text { non-steroids }\end{array}$} \\
\hline 2005 & 54.9 & 40.3 & 24.0 & 76.7 & 55.8 & 68.0 & 44.0 & 51.4 \\
\hline 2010 & 53.4 & 36.9 & 24.4 & 83.3 & 56.1 & 75.7 & 45.5 & 54.3 \\
\hline 2013 & 42.4 & 33.2 & 24.0 & 80.7 & 55.8 & 76.1 & 46.9 & 53.6 \\
\hline 2014 & 40.6 & 33.9 & 19.9 & 78.8 & 53.6 & 74.6 & 45.9 & 52.3 \\
\hline 2015 & 40.7 & 32.9 & 21.3 & 77.0 & 55.7 & 77.2 & 47.0 & 54.3 \\
\hline \multicolumn{9}{|l|}{ N02A } \\
\hline \multicolumn{9}{|c|}{ Opioids } \\
\hline 2005 & 18.5 & 6.9 & 4.5 & 15.1 & 9.1 & 17.4 & 19.5 & 20.8 \\
\hline 2010 & 20.2 & 7.8 & 6.5 & 16.5 & 9.1 & 19.0 & 19.8 & 20.0 \\
\hline 2013 & 20.1 & 7.6 & 7.1 & 16.3 & 9.6 & 20.6 & 19.3 & 18.2 \\
\hline 2014 & 20.4 & 8.2 & 7.3 & 15.9 & 9.8 & 20.7 & 18.9 & 17.7 \\
\hline 2015 & 20.3 & 8.0 & 5.9 & 15.6 & 10.2 & 21.4 & 18.8 & 17.0 \\
\hline \multicolumn{9}{|l|}{ N02B } \\
\hline \multicolumn{9}{|c|}{$\begin{array}{l}\text { Other analgesics } \\
\text { and antipyretics }\end{array}$} \\
\hline 2005 & 71.2 & 54.7 & 44.3 & 20.6 & 36.3 & 30.9 & 29.8 & 49.5 \\
\hline 2010 & 74.0 & 58.3 & 43.6 & 29.5 & 44.3 & 33.9 & 34.5 & 52.4 \\
\hline 2013 & 72.9 & 55.2 & 42.9 & 31.9 & 43.2 & 36.9 & 38.2 & 55.7 \\
\hline 2014 & 66.5 & 51.6 & 46.1 & 35.1 & 43.2 & 38.8 & 37.9 & 54.4 \\
\hline 2015 & 71.3 & 56.5 & 47.8 & 34.3 & 45.2 & 41.0 & 38.6 & 55.0 \\
\hline \multicolumn{9}{|c|}{ N02BA } \\
\hline \multicolumn{9}{|c|}{$\begin{array}{l}\text { Salicylic acid } \\
\text { and derivatives }\end{array}$} \\
\hline 2005 & 12.9 & 14.3 & 0.8 & 5.5 & 9.9 & 3.5 & 0.5 & 9.8 \\
\hline 2010 & 8.9 & 11.1 & 0.2 & 3.6 & 7.7 & 2.9 & 0.3 & 8.0 \\
\hline 2013 & 7.5 & 8.4 & 0.1 & 2.6 & 6.9 & 3.3 & 0.2 & 7.5 \\
\hline 2014 & 6.0 & 7.2 & 0.1 & 2.4 & 6.9 & 3.3 & 0.2 & 7.1 \\
\hline 2015 & 6.1 & 7.7 & 0.2 & 2.2 & 6.5 & 3.6 & 0.2 & 7.0 \\
\hline \multicolumn{9}{|c|}{ N02BB } \\
\hline \multicolumn{9}{|c|}{ Pyrazolones } \\
\hline 2005 & 0.6 & - & - & - & - & - & 3.2 & 0.1 \\
\hline 2010 & 0.3 & - & - & - & - & - & 2.3 & 0.1 \\
\hline 2013 & 0.2 & - & - & - & - & - & 1.8 & 0.1 \\
\hline 2014 & 0.1 & - & - & - & - & - & 1.6 & 0.1 \\
\hline 2015 & - & - & - & - & - & - & 1.5 & - \\
\hline \multicolumn{9}{|c|}{ N02BE } \\
\hline \multicolumn{9}{|c|}{ Anilides } \\
\hline 2005 & 57.7 & 40.3 & 24.6 & 15.1 & 26.4 & 27.4 & 26.0 & 39.7 \\
\hline 2010 & 64.8 & 47.2 & 43.4 & 25.9 & 36.6 & 31.0 & 31.9 & 44.3 \\
\hline 2013 & 65.0 & 46.8 & 42.8 & 29.3 & 36.3 & 33.6 & 36.2 & 48.1 \\
\hline 2014 & 60.4 & 44.5 & 46.0 & 32.7 & 36.3 & 35.5 & 36.0 & 47.2 \\
\hline 2015 & 65.1 & 48.8 & 47.6 & 32.1 & 38.7 & 37.3 & 36.9 & 47.9 \\
\hline
\end{tabular}

1 Sales of OTC medicines in the group N02BE for 2005 and 2006 in Greenland are not available 2 Includes data on OTC drugs sold outside of pharmacies 
Figure 3.7.7 Sales of non-opioid analgesics (ATC-groups M01A and N02B), DDD/1 000 inhabitants/day, 2005-2015

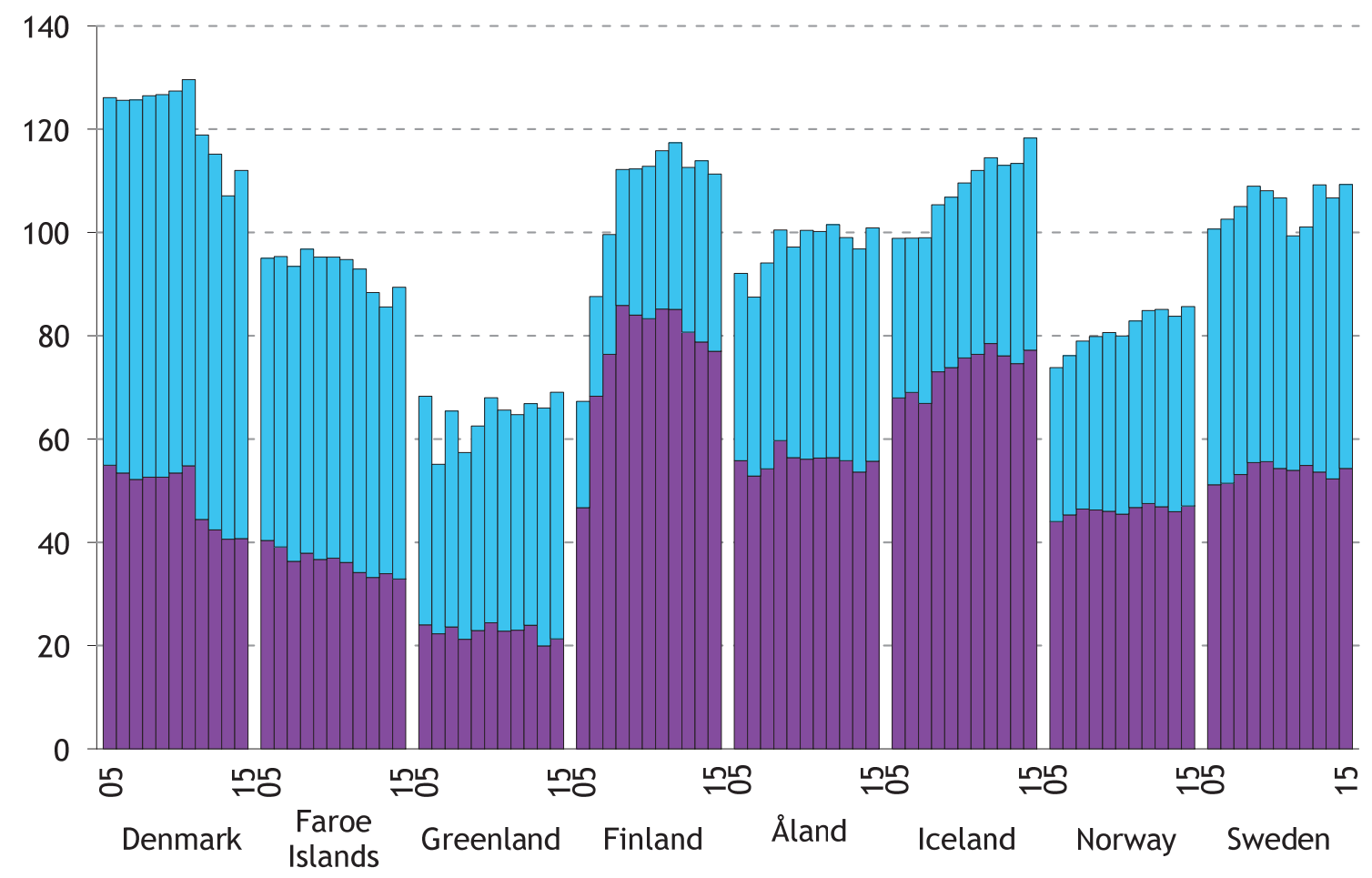

M01A Antiinflammatory and antiheumatic products, non-steroids

$\square$ N02B Other analgesics and antipyretics 
Figure 3.7.8 Sales of opioid analgesics (ATC-group N02A), DDD/1 000 inhabitants/day, 2005-2015

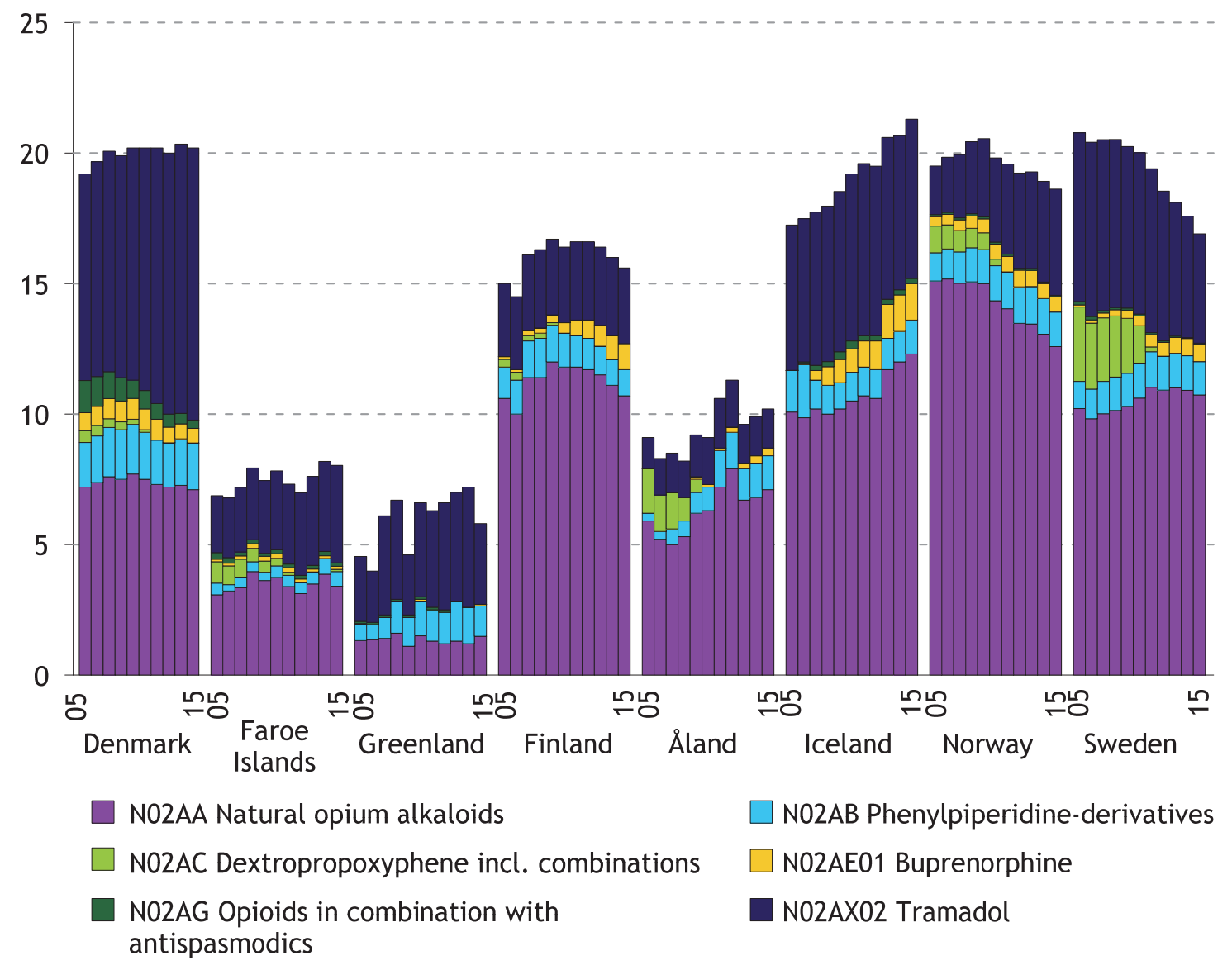

Table 3.7.25 Sales of antimigraine preparations (ATC-group N02C), DDD/1 000 inhabitants/day, 2005-2015

\begin{tabular}{lcccccccc}
\hline & Denmark & $\begin{array}{c}\text { Faroe } \\
\text { Islands }\end{array}$ & Greenland & Finland & Åland & Iceland & Norway & Sweden \\
\hline 2005 & 2.5 & 2.1 &.. & 1.1 & 2.0 & 1.5 & 3.2 & 3.1 \\
2010 & 2.9 & 2.4 & 1.3 & 1.5 & 2.2 & 1.7 & 3.5 & 3.0 \\
2013 & 3.0 & 2.3 & 1.4 & 1.9 & 2.1 & 1.7 & 3.7 & 3.0 \\
2014 & 3.1 & 2.3 & 1.5 & 2.0 & 1.9 & 1.8 & 3.8 & 2.9 \\
2015 & 3.2 & 2.4 & 1.5 & 2.0 & 1.9 & 1.9 & 4.0 & 3.0 \\
\hline
\end{tabular}

Table 3.7.26 Sales of anti-epileptics (ATC-group N03), DDD/1 000 inhabitants/day, 2005-2015

\begin{tabular}{lrrrrrrrr}
\hline & Denmark & $\begin{array}{c}\text { Faroe } \\
\text { Islands }\end{array}$ & Greenland & Finland & Åland & Iceland & Norway & Sweden \\
\hline 2005 & 11.6 & 9.0 & & & & & & \\
2010 & 14.7 & 11.5 & 9.7 & 12.5 & 8.4 & 11.9 & 10.2 & 9.1 \\
2013 & 17.3 & 13.8 & 10.6 & 19.9 & 11.2 & 17.2 & 14.5 & 13.0 \\
2014 & 18.0 & 14.5 & 10.9 & 19.9 & 12.5 & 18.9 & 15.6 & 14.2 \\
2015 & 18.7 & 15.8 & 11.5 & 20.1 & 13.7 & 20.5 & 16.7 & 14.4 \\
\hline
\end{tabular}


Table 3.7.27 Sales of antiparkinson drugs (ATC-group N04), DDD/1 000 inhabitants/day, 2005-2015

\begin{tabular}{lcccccccc}
\hline & Denmark & $\begin{array}{c}\text { Faroe } \\
\text { Islands }\end{array}$ & Greenland & Finland & Åland & Iceland & Norway & Sweden \\
\hline 2005 & 3.5 & 5.0 &.. & 4.5 & 2.9 & 4.1 & 3.0 & 3.7 \\
2010 & 4.1 & 4.4 & 2.7 & 4.9 & 3.7 & 4.8 & 3.5 & 4.1 \\
2013 & 4.4 & 4.3 & 3.0 & 4.7 & 3.8 & 4.6 & 3.8 & 4.2 \\
2014 & 4.4 & 4.6 & 3.1 & 5.2 & 3.8 & 4.5 & 3.8 & 4.2 \\
2015 & 4.5 & 4.9 & 3.1 & 5.3 & 3.6 & 4.6 & 3.9 & 4.3 \\
\hline
\end{tabular}

Table 3.7.28 Sales of antipsychotics (ATC-group N05A), DDD/1 000 inhabitants/day, 2005-2015

\begin{tabular}{lcccccccc}
\hline & Denmark & $\begin{array}{c}\text { Faroe } \\
\text { Islands }\end{array}$ & Greenland & Finland & Åland & Iceland & Norway & Sweden \\
\hline 2005 & 13.0 & 10.4 & 14.6 & 17.4 & 9.3 & 11.5 & 10.6 & 9.2 \\
2010 & 13.9 & 12.7 & 16.0 & 20.7 & 9.6 & 11.1 & 10.8 & 9.8 \\
2013 & 14.3 & 12.8 & 15.7 & 21.5 & 9.2 & 12.4 & 10.9 & 10.4 \\
2014 & 14.1 & 13.0 & 15.4 & 21.8 & 9.6 & 12.6 & 10.9 & 10.4 \\
2015 & 13.9 & 12.8 & 16.0 & 21.8 & 9.7 & 13.0 & 11.0 & 10.6 \\
\hline
\end{tabular}

Table 3.7.29 Sales of anxiolytics (ATC-group N05B), DDD/1 000 inhabitants/day, 2005-2015

\begin{tabular}{|c|c|c|c|c|c|c|c|c|}
\hline & Denmark & $\begin{array}{l}\text { Faroe } \\
\text { Islands }\end{array}$ & Greenland & Finland & Åland & Iceland & Norway & Sweden \\
\hline \multicolumn{9}{|c|}{ N05B } \\
\hline \multicolumn{9}{|c|}{ Anxiolytics } \\
\hline 2005 & 19.9 & 17.1 & 5.3 & 31.2 & 9.9 & 25.8 & 21.3 & 16.4 \\
\hline 2010 & 11.8 & 11.5 & 2.9 & 28.0 & 10.7 & 24.6 & 19.5 & 16.2 \\
\hline 2013 & 9.6 & 10.2 & 2.3 & 24.3 & 10.5 & 23.2 & 16.4 & 15.4 \\
\hline 2014 & 9.0 & 10.1 & 2.5 & 23.0 & 11.1 & 22.8 & 15.5 & 15.0 \\
\hline 2015 & 8.3 & 9.6 & 2.0 & 21.6 & 12.3 & 22.6 & 14.4 & 14.5 \\
\hline \multicolumn{9}{|c|}{ N05BA } \\
\hline \multicolumn{9}{|c|}{$\begin{array}{l}\text { Benzodiazepine } \\
\text { derivates }\end{array}$} \\
\hline 2005 & 19.6 & 17.0 & 5.3 & 29.5 & 8.0 & 24.6 & 20.1 & 13.6 \\
\hline 2010 & 11.5 & 11.2 & 2.1 & 26.2 & 8.5 & 23.2 & 18.0 & 12.8 \\
\hline 2013 & 9.3 & 9.9 & 2.3 & 22.4 & 7.9 & 21.6 & 14.8 & 11.7 \\
\hline 2014 & 8.7 & 9.9 & 2.5 & 21.1 & 8.3 & 21.2 & 14.0 & 11.2 \\
\hline 2015 & 7.9 & 9.3 & 2.0 & 19.8 & 9.5 & 21.0 & 12.9 & 10.6 \\
\hline
\end{tabular}


Morbidity, Medical Treatment, Accidents and Pharmaceutical products

Table 3.7.30 Sales of hypnotics and sedatives (ATC-group N05C), DDD/1 000 inhabitants/day, 2005-2015

\begin{tabular}{|c|c|c|c|c|c|c|c|c|}
\hline & Denmark & $\begin{array}{l}\text { Faroe } \\
\text { Islands }\end{array}$ & Greenland & Finland & Åland & Iceland & Norway & Sweden \\
\hline \multicolumn{9}{|l|}{ N05C } \\
\hline \multicolumn{9}{|c|}{ Hypnotics and sedatives } \\
\hline 2005 & 31.4 & 30.5 & 8.8 & 54.4 & 34.2 & 66.7 & 41.4 & 51.1 \\
\hline 2010 & 20.1 & 22.3 & 7.1 & 46.8 & 34.3 & 75.9 & 42.5 & 52.2 \\
\hline 2013 & 16.7 & 18.2 & 5.7 & 39.2 & 33.8 & 71.8 & 39.0 & 52.5 \\
\hline 2014 & 16.3 & 17.5 & 4.6 & 36.1 & 35.0 & 69.9 & 38.1 & 51.8 \\
\hline 2015 & 15.3 & 16.6 & 5.8 & 32.9 & 34.4 & 69.3 & 36.7 & 50.8 \\
\hline \multicolumn{9}{|c|}{ N05CD } \\
\hline \multicolumn{9}{|c|}{ Benzodiazepine derivates } \\
\hline 2005 & 10.5 & 7.1 & 0.5 & 20.9 & 4.3 & 12.1 & 8.5 & 6.7 \\
\hline 2010 & 4.8 & 4.2 & 0.4 & 15.6 & 3.5 & 8.6 & 6.3 & 4.1 \\
\hline 2013 & 2.9 & 2.6 & 0.3 & 12.0 & 3.1 & 6.0 & 3.6 & 3.0 \\
\hline 2014 & 2.5 & 2.4 & 0.1 & 10.7 & 3.2 & 5.4 & 3.3 & 2.7 \\
\hline 2015 & 2.2 & 2.1 & 0.2 & 9.5 & 3.2 & 4.8 & 3.1 & 2.4 \\
\hline \multicolumn{9}{|c|}{ N05CF } \\
\hline \multicolumn{9}{|c|}{$\begin{array}{l}\text { Benzodiazepine-related } \\
\text { drugs }\end{array}$} \\
\hline 2005 & 20.9 & 23.4 & 8.3 & 33.1 & 29.3 & 54.5 & 32.8 & 30.4 \\
\hline 2010 & 15.3 & 18.1 & 6.7 & 30.7 & 30.3 & 66.2 & 36.1 & 34.1 \\
\hline 2013 & 13.8 & 15.6 & 5.4 & 26.9 & 30.3 & 63.1 & 35.2 & 35.9 \\
\hline 2014 & 13.7 & 15.1 & 4.5 & 25.1 & 31.4 & 61.1 & 34.7 & 36.0 \\
\hline 2015 & 13.1 & 14.5 & 5.6 & 23.2 & 30.9 & 60.3 & 33.5 & 35.4 \\
\hline
\end{tabular}

1 Sales excluding melatonin ( $\mathrm{N05CH01)}$ 
Morbidity, Medical Treatment, Accidents and Pharmaceutical products

Figure 3.7.9 Sales of hypnotics and sedatives (ATC groups N05CD and N05CF), DDD/1000 inhabitants/day, 2005-2015

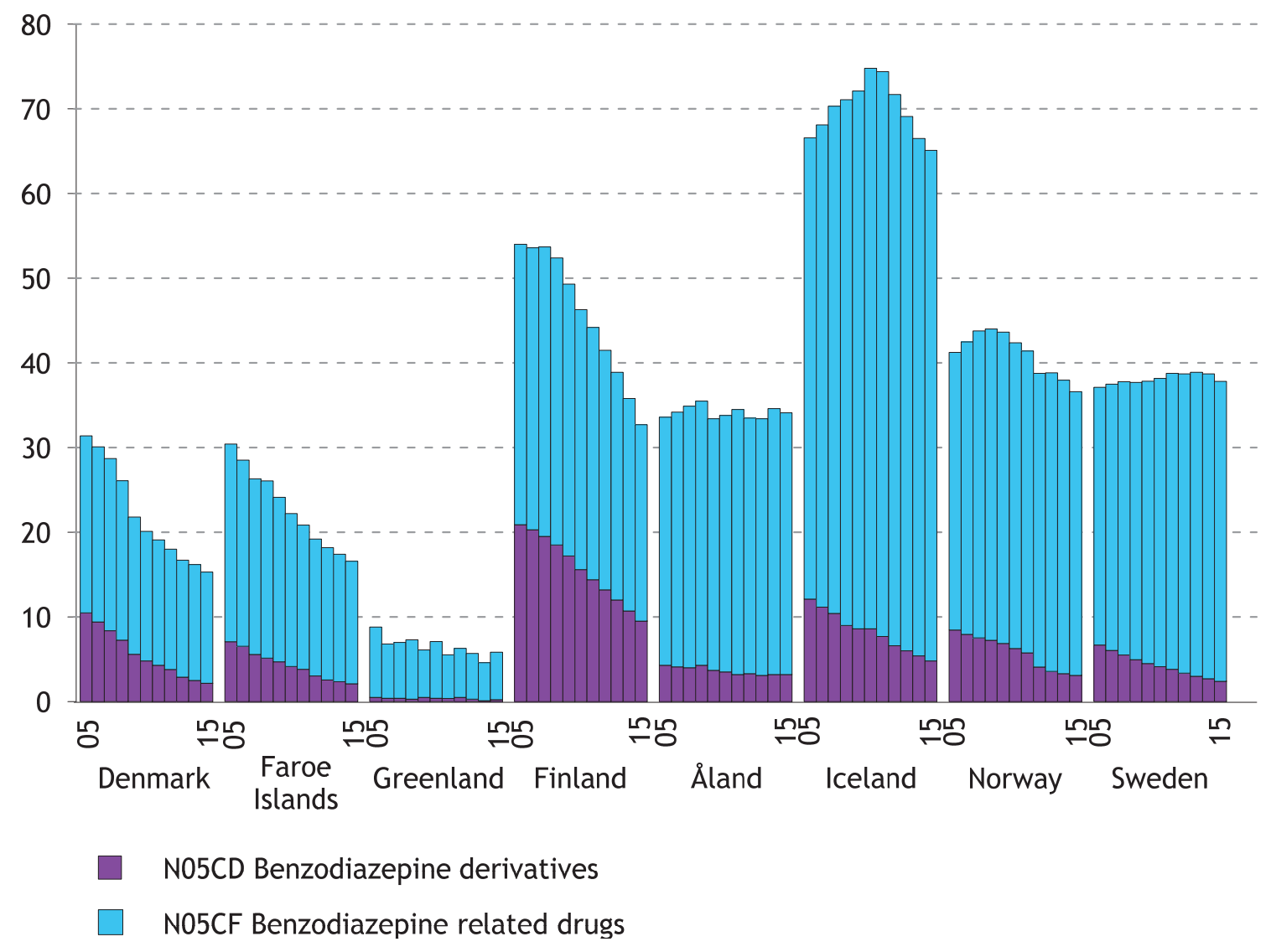


Morbidity, Medical Treatment, Accidents and Pharmaceutical products

Table 3.7.31 Sales of antidepressants (ATC-group N06A), DDD/1 000 inhabitants/day, 2005-2015

\begin{tabular}{|c|c|c|c|c|c|c|c|c|}
\hline & Denmark & $\begin{array}{c}\text { Faroe } \\
\text { Islands }\end{array}$ & Greenland & Finland & Åland & Iceland & Norway & Sweden \\
\hline \multicolumn{9}{|c|}{$\begin{array}{l}\text { N06A } \\
\text { Antidepressants }\end{array}$} \\
\hline $\begin{array}{l}2005 \\
2010 \\
2013 \\
2014 \\
2015\end{array}$ & $\begin{array}{l}60.1 \\
84.0 \\
80.0 \\
76.7 \\
77.0\end{array}$ & $\begin{array}{l}36.5 \\
57.2 \\
62.6 \\
61.8 \\
61.5\end{array}$ & $\begin{array}{l}20.4 \\
24.1 \\
25.5 \\
23.3 \\
23.0\end{array}$ & $\begin{array}{l}52.1 \\
69.2 \\
69.4 \\
68.8 \\
68.2\end{array}$ & $\begin{array}{l}40.7 \\
50.9 \\
56.3 \\
61.0 \\
60.2\end{array}$ & $\begin{array}{r}94.8 \\
100.9 \\
117.7 \\
119.1 \\
129.6\end{array}$ & $\begin{array}{l}51.8 \\
56.4 \\
56.1 \\
55.7 \\
56.5\end{array}$ & $\begin{array}{l}66.1 \\
75.8 \\
84.3 \\
87.8 \\
92.5\end{array}$ \\
\hline \multicolumn{9}{|c|}{$\begin{array}{l}\text { N06AA } \\
\text { Non-selective monoamine } \\
\text { reuptake inhibitors }\end{array}$} \\
\hline $\begin{array}{l}2005 \\
2010 \\
2013 \\
2014 \\
2015\end{array}$ & $\begin{array}{l}4.3 \\
4.6 \\
4.6 \\
4.5 \\
4.4\end{array}$ & $\begin{array}{l}2.1 \\
1.9 \\
1.6 \\
1.7 \\
1.7\end{array}$ & $\begin{array}{l}1.1 \\
0.8 \\
1.2 \\
1.1 \\
1.2\end{array}$ & $\begin{array}{l}4.2 \\
4.3 \\
4.3 \\
4.2 \\
4.2\end{array}$ & $\begin{array}{l}3.2 \\
2.9 \\
3.1 \\
3.2 \\
3.5\end{array}$ & $\begin{array}{l}8.1 \\
5.5 \\
5.6 \\
5.5 \\
5.4\end{array}$ & $\begin{array}{l}3.8 \\
3.6 \\
3.5 \\
3.5 \\
3.5\end{array}$ & $\begin{array}{l}3.8 \\
3.5 \\
3.2 \\
3.3 \\
3.4\end{array}$ \\
\hline \multicolumn{9}{|c|}{$\begin{array}{l}\text { N06AB } \\
\text { Selective serotonin } \\
\text { reuptake inhibitors }\end{array}$} \\
\hline $\begin{array}{l}2005 \\
2010 \\
2013 \\
2014 \\
2015\end{array}$ & $\begin{array}{l}41.7 \\
56.6 \\
50.1 \\
47.5 \\
47.7\end{array}$ & $\begin{array}{l}26.4 \\
40.5 \\
44.0 \\
43.3 \\
42.8\end{array}$ & $\begin{array}{l}16.0 \\
16.6 \\
16.8 \\
15.7 \\
14.1\end{array}$ & $\begin{array}{l}35.3 \\
44.2 \\
41.7 \\
40.8 \\
39.8\end{array}$ & $\begin{array}{l}30.4 \\
36.1 \\
36.7 \\
39.9 \\
40.8\end{array}$ & $\begin{array}{l}64.8 \\
72.3 \\
84.0 \\
86.5 \\
94.2\end{array}$ & $\begin{array}{l}34.8 \\
37.8 \\
36.9 \\
36.4 \\
36.9\end{array}$ & $\begin{array}{l}48.4 \\
53.2 \\
57.8 \\
59.8 \\
62.5\end{array}$ \\
\hline \multicolumn{9}{|c|}{$\begin{array}{l}\text { N06AG } \\
\text { Monoamine oxidase type } \\
\text { A inhibitors }\end{array}$} \\
\hline $\begin{array}{l}2005 \\
2010 \\
2013 \\
2014 \\
2015\end{array}$ & $\begin{array}{r}0.1 \\
- \\
- \\
- \\
-\end{array}$ & $\begin{array}{l}- \\
- \\
- \\
- \\
-\end{array}$ & $\begin{array}{l}- \\
- \\
- \\
- \\
-\end{array}$ & $\begin{array}{l}0.7 \\
0.6 \\
0.4 \\
0.4 \\
0.4\end{array}$ & $\begin{array}{l}0.2 \\
0.3 \\
0.1 \\
0.1 \\
0.1\end{array}$ & $\begin{array}{l}0.8 \\
0.5 \\
0.4 \\
0.4 \\
0.3\end{array}$ & $\begin{array}{l}0.3 \\
0.2 \\
0.1 \\
0.1 \\
0.1\end{array}$ & $\begin{array}{l}0.2 \\
0.1 \\
0.1 \\
0.1 \\
0.1\end{array}$ \\
\hline \multicolumn{9}{|c|}{$\begin{array}{l}\text { N06AX } \\
\text { Other antidepressants }\end{array}$} \\
\hline $\begin{array}{l}2005 \\
2010 \\
2013 \\
2014 \\
2015\end{array}$ & $\begin{array}{l}13.9 \\
22.7 \\
25.0 \\
24.5 \\
24.8\end{array}$ & $\begin{array}{r}8.0 \\
14.8 \\
17.1 \\
16.8 \\
17.0 \\
\end{array}$ & $\begin{array}{l}3.3 \\
6.6 \\
7.5 \\
6.6 \\
7.7 \\
\end{array}$ & $\begin{array}{l}12.0 \\
20.2 \\
23.0 \\
23.4 \\
23.8\end{array}$ & $\begin{array}{r}6.8 \\
11.5 \\
16.3 \\
17.8 \\
15.8 \\
\end{array}$ & $\begin{array}{l}21.2 \\
22.6 \\
27.7 \\
26.8 \\
29.7 \\
\end{array}$ & $\begin{array}{l}13.0 \\
14.8 \\
15.5 \\
15.7 \\
15.9 \\
\end{array}$ & $\begin{array}{l}13.6 \\
19.0 \\
23.2 \\
24.7 \\
26.6\end{array}$ \\
\hline
\end{tabular}


Figure 3.7.10 Sales of antidepressants (ATC-group N06A), DDD/1 000 inhabitants/day, 2005-2015

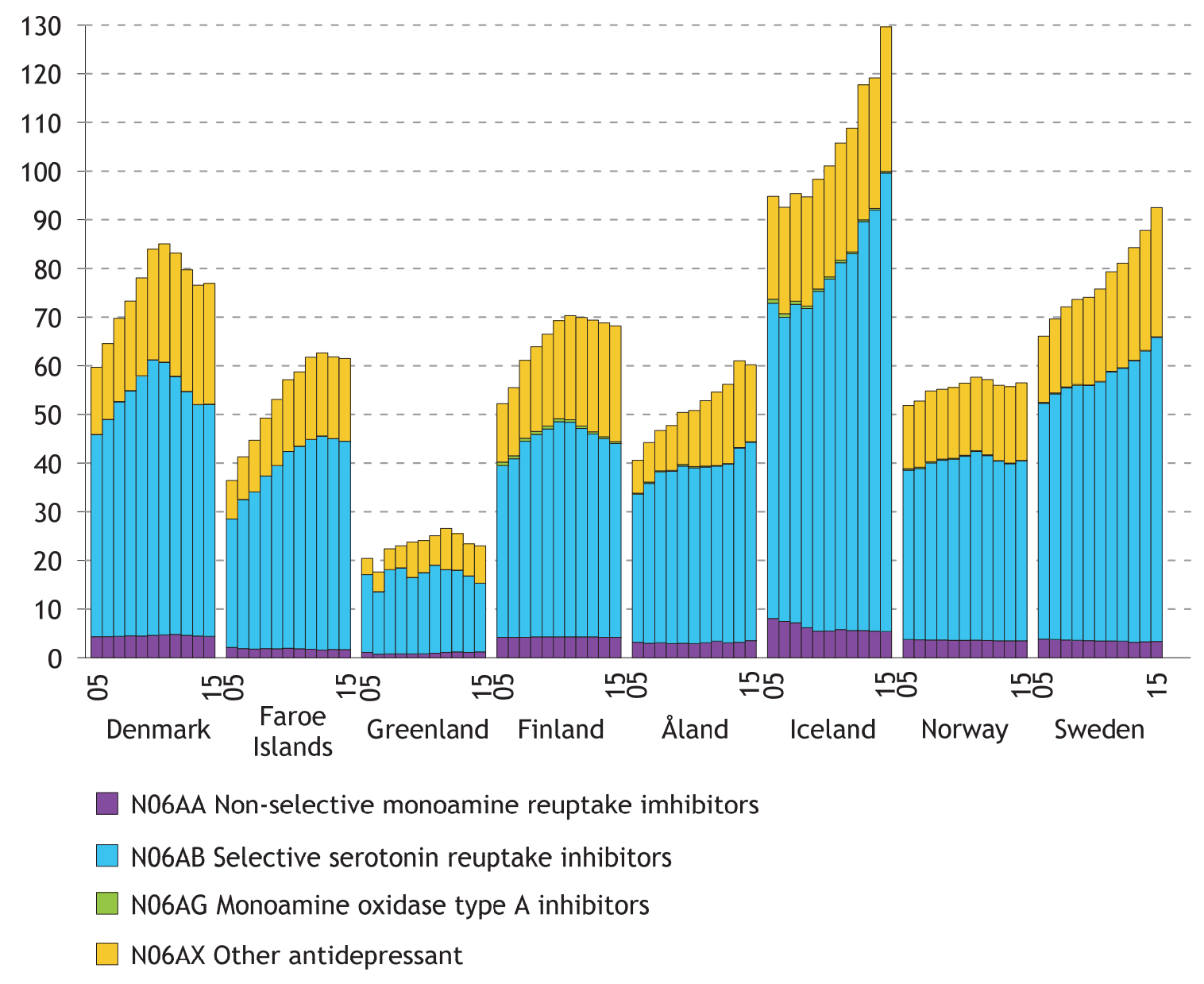


Morbidity, Medical Treatment, Accidents and Pharmaceutical products

Table 3.7.32 Proportion of the population per 1000 by age and gender (oneyear prevalence) receiving at least one antidepressant (ATC-group N06A), 2015

\begin{tabular}{|c|c|c|c|}
\hline & Men & Women & Total \\
\hline \multicolumn{4}{|c|}{ Denmark } \\
\hline $0-14$ & 1 & 1 & 1 \\
\hline $15-24$ & 20 & 43 & 31 \\
\hline $25-44$ & 53 & 91 & 72 \\
\hline $45-64$ & 74 & 124 & 99 \\
\hline $65-74$ & 84 & 129 & 107 \\
\hline $75+$ & 134 & 212 & 180 \\
\hline Total & .. & .. & .. \\
\hline \multicolumn{4}{|c|}{ Faroe Islands } \\
\hline $0-14$ & 1 & 1 & 1 \\
\hline $15-24$ & 23 & 45 & 33 \\
\hline $25-44$ & 46 & 75 & 60 \\
\hline $45-64$ & 52 & 95 & 73 \\
\hline $65-74$ & 76 & 136 & 105 \\
\hline $75+$ & 153 & 252 & 210 \\
\hline Total & 44 & 82 & 62 \\
\hline \multicolumn{4}{|c|}{ Finland } \\
\hline $0-14$ & 2 & 2 & .. \\
\hline $15-24$ & 33 & 68 & .. \\
\hline $25-44$ & 67 & 104 & .. \\
\hline $45-64$ & 77 & 124 & .. \\
\hline $65-74$ & 69 & 108 & .. \\
\hline $75+$ & 111 & 172 & .. \\
\hline Total & .. & .. & .. \\
\hline \multicolumn{4}{|c|}{ Iceland } \\
\hline $0-14$ & 23 & 20 & 22 \\
\hline $15-24$ & 93 & 182 & 137 \\
\hline $25-44$ & 121 & 216 & 167 \\
\hline $45-64$ & 136 & 264 & 200 \\
\hline $65-74$ & 164 & 316 & 246 \\
\hline $75+$ & 220 & 342 & 289 \\
\hline Total & .. & .. & .. \\
\hline \multicolumn{4}{|c|}{ Norway } \\
\hline $0-14$ & 1 & 1 & 1 \\
\hline $15-24$ & 20 & 41 & 30 \\
\hline $25-44$ & 44 & 77 & 60 \\
\hline $45-64$ & 63 & 121 & 91 \\
\hline $65-74$ & 67 & 131 & 99 \\
\hline $75+$ & 84 & 153 & 125 \\
\hline Total & 43 & 83 & 63 \\
\hline \multicolumn{4}{|c|}{ Sweden } \\
\hline $0-14$ & 3 & 3 & 3 \\
\hline $15-24$ & 37 & 70 & 53 \\
\hline $25-44$ & 68 & 128 & 97 \\
\hline $45-64$ & 85 & 160 & 122 \\
\hline $65-74$ & 88 & 156 & 123 \\
\hline $75+$ & 154 & 250 & 210 \\
\hline Total & .. & .. & .. \\
\hline
\end{tabular}


Morbidity, Medical Treatment, Accidents and Pharmaceutical products

Table 3.7.33 Proportion of the population per 1000 by age $0-39$ and gender (one-year prevalence) receiving at least one drug used in the treatment of e.g. Attention Deficit Hyperactivity Disorder (ATC group N06BA1, centrally acting sympathomimetic), 2015

\begin{tabular}{|c|c|c|c|}
\hline & Men & Women & Total \\
\hline \multicolumn{4}{|c|}{ Denmark } \\
\hline $0-4$ & & - & - \\
\hline $5-9$ & 13 & 4 & 8 \\
\hline $10-14$ & 34 & 11 & 23 \\
\hline $15-19$ & 26 & 16 & 21 \\
\hline $20-24$ & 16 & 13 & 14 \\
\hline $25-29$ & 14 & 10 & 12 \\
\hline $30-39$ & 10 & 8 & 9 \\
\hline \multicolumn{4}{|c|}{ Faroe Islands } \\
\hline $0-4$ & - & - & - \\
\hline $5-9$ & 10 & 1 & 5 \\
\hline $10-14$ & 25 & 5 & 15 \\
\hline $15-19$ & 20 & 16 & 18 \\
\hline $20-24$ & 17 & 17 & 17 \\
\hline $25-29$ & 10 & 9 & 10 \\
\hline $30-39$ & 7 & 6 & 7 \\
\hline \multicolumn{4}{|c|}{ Finland } \\
\hline $0-4$ & - & - & - \\
\hline $5-9$ & 18 & 4 & 11 \\
\hline $10-14$ & 40 & 8 & 24 \\
\hline $15-19$ & 17 & 6 & 12 \\
\hline $20-24$ & 5 & 3 & 4 \\
\hline $25-29$ & 4 & 3 & 3 \\
\hline $30-39$ & 3 & 3 & 3 \\
\hline \multicolumn{4}{|c|}{ Iceland } \\
\hline $0-4$ & - & - & - \\
\hline $5-9$ & 56 & 20 & 38 \\
\hline $10-14$ & 131 & 52 & 92 \\
\hline $15-19$ & 86 & 51 & 69 \\
\hline $20-24$ & 37 & 32 & 34 \\
\hline $25-29$ & 35 & 30 & 33 \\
\hline $30-39$ & 29 & 29 & 29 \\
\hline \multicolumn{4}{|c|}{ Norway } \\
\hline $0-4$ & & - & - \\
\hline $5-9$ & 12 & 4 & 8 \\
\hline $10-14$ & 39 & 15 & 27 \\
\hline $15-19$ & 29 & 17 & 23 \\
\hline $20-24$ & 14 & 12 & 13 \\
\hline $25-29$ & 10 & 11 & 10 \\
\hline $30-39$ & 8 & 8 & 8 \\
\hline \multirow{2}{*}{\multicolumn{4}{|c|}{$\begin{array}{l}\text { Sweden } \\
0-4\end{array}$}} \\
\hline & & & \\
\hline $5-9$ & 14 & 5 & 10 \\
\hline $10-14$ & 54 & 19 & 37 \\
\hline $15-19$ & 45 & 28 & 37 \\
\hline $20-24$ & 17 & 17 & 17 \\
\hline $25-29$ & 14 & 13 & 14 \\
\hline $30-39$ & 11 & 10 & 10 \\
\hline
\end{tabular}

1 Excl. N06BA07 modafinil 
Morbidity, Medical Treatment, Accidents and Pharmaceutical products

Table 3.7.34 Proportion of the population per 1000 aged 0-39 (one-year prevalence) receiving at least one centrally acting sympathomimetic (ATC group N06BA ${ }^{1}$ )

\begin{tabular}{|c|c|c|c|}
\hline & Men & Women & Total \\
\hline \multicolumn{4}{|c|}{ Denmark } \\
\hline 2005 & .. & .. & 2 \\
\hline 2010 & .. & .. & 10 \\
\hline 2013 & .. & .. & \\
\hline 2014 & .. & .. & 12 \\
\hline 2015 & 16 & 9 & 12 \\
\hline \multicolumn{4}{|c|}{ Faroe Islands } \\
\hline 2005 & 2 & - & 1 \\
\hline 2010 & 3 & 7 & 5 \\
\hline 2013 & 11 & 6 & 8 \\
\hline 2014 & 11 & 7 & 9 \\
\hline 2015 & 13 & 7 & 10 \\
\hline \multicolumn{4}{|c|}{ Finland } \\
\hline 2005 & .. & .. & 1 \\
\hline 2010 & .. & .. & 5 \\
\hline 2013 & .. & .. & \\
\hline 2014 & .. & .. & 9 \\
\hline 2015 & .. & .. & 10 \\
\hline \multicolumn{4}{|c|}{ Iceland } \\
\hline 2005 & .. & .. & 10 \\
\hline 2010 & .. & .. & 16 \\
\hline 2013 & .. & .. & \\
\hline 2014 & .. & .. & 23 \\
\hline 2015 & 43 & 28 & 36 \\
\hline \multicolumn{4}{|c|}{ Norway } \\
\hline 2005 & .. & .. & 6 \\
\hline 2010 & .. & .. & 10 \\
\hline 2013 & .. & .. & \\
\hline 2014 & .. & .. & 12 \\
\hline 2015 & 15 & 9 & 12 \\
\hline \multicolumn{4}{|c|}{ Sweden } \\
\hline 2005 & .. & .. & .. \\
\hline 2010 & 11 & 6 & 9 \\
\hline 2013 & 17 & 10 & 13 \\
\hline 2014 & 18 & 11 & 15 \\
\hline 2015 & 20 & 12 & 16 \\
\hline
\end{tabular}

1 Excl. N06BA07

Table 3.7.35 Sales of anti-dementia drugs (ATC-group N06D), DDD/1 000 inhabitants/day, 2005-2015

\begin{tabular}{lcccccccc}
\hline & Denmark & $\begin{array}{c}\text { Faroe } \\
\text { Islands }\end{array}$ & Greenland & Finland & Åland & Iceland & Norway & Sweden \\
\hline 2005 & 2.0 & 1.1 & 0.1 & 6.5 & 2.5 & 2.7 & 3.1 & 3.0 \\
2010 & 2.9 & 2.3 & 0.2 & 12.1 & 4.5 & 2.9 & 3.0 & 3.6 \\
2013 & 3.4 & 4.2 & 0.6 & 15.3 & 3.9 & 3.7 & 3.3 & 4.2 \\
2014 & 3.7 & 4.9 & 0.4 & 15.6 & 3.8 & 3.8 & 2.9 & 4.5 \\
2015 & 3.9 & 5.2 & 0.4 & 15.8 & 3.4 & 3.7 & 2.7 & 4.8 \\
\hline
\end{tabular}


Table 3.7.36 Sales of nasal preparations, decongestants and other preparations for local use - (ATC group R01A),

DDD/1 000 inhabitants/day, 2005-2015

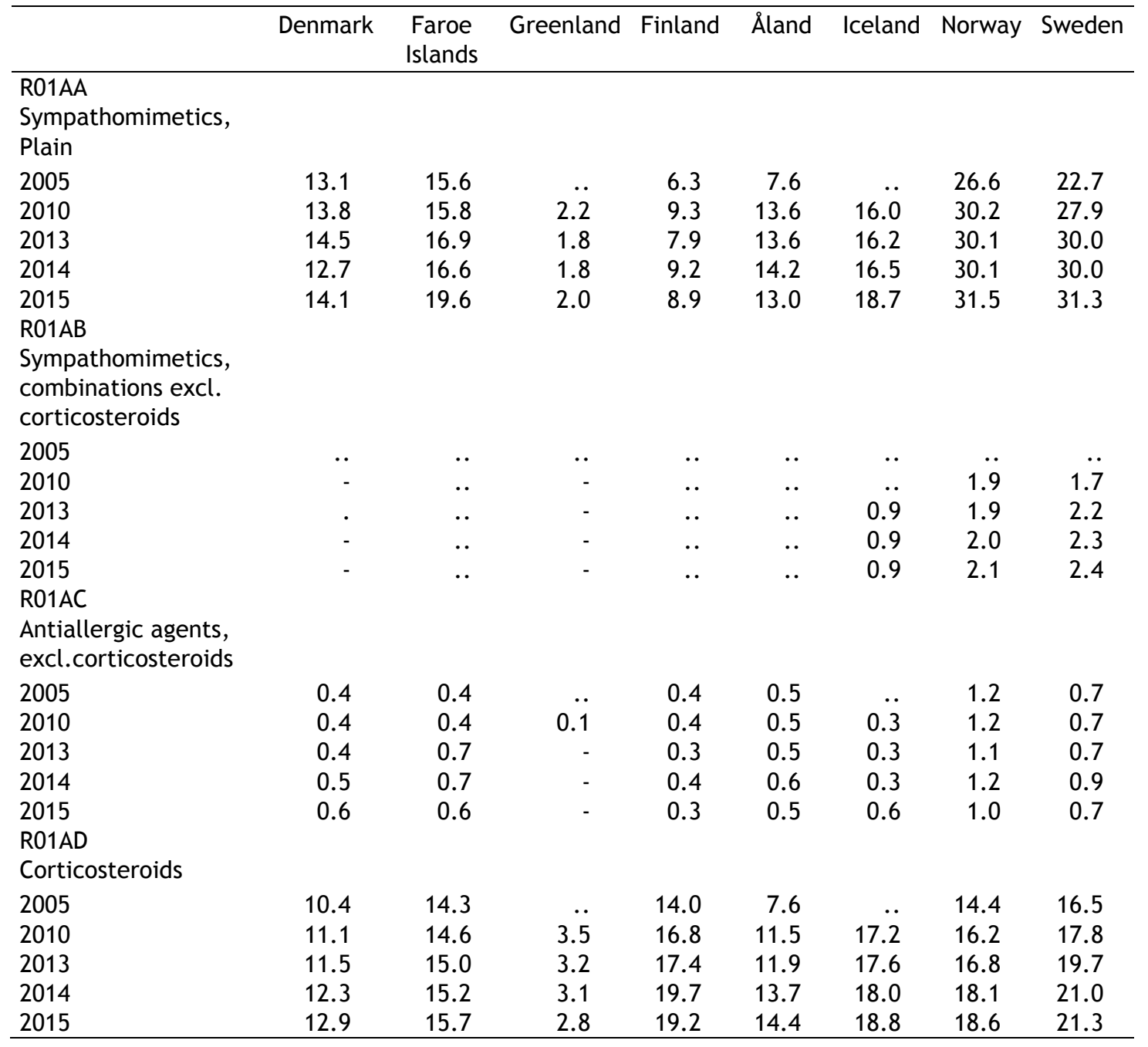


Table 3.7.37 Sales of drugs for obstructive airway diseases (ATC-group R03), DDD/1 000 inhabitants/day, 2005-2015

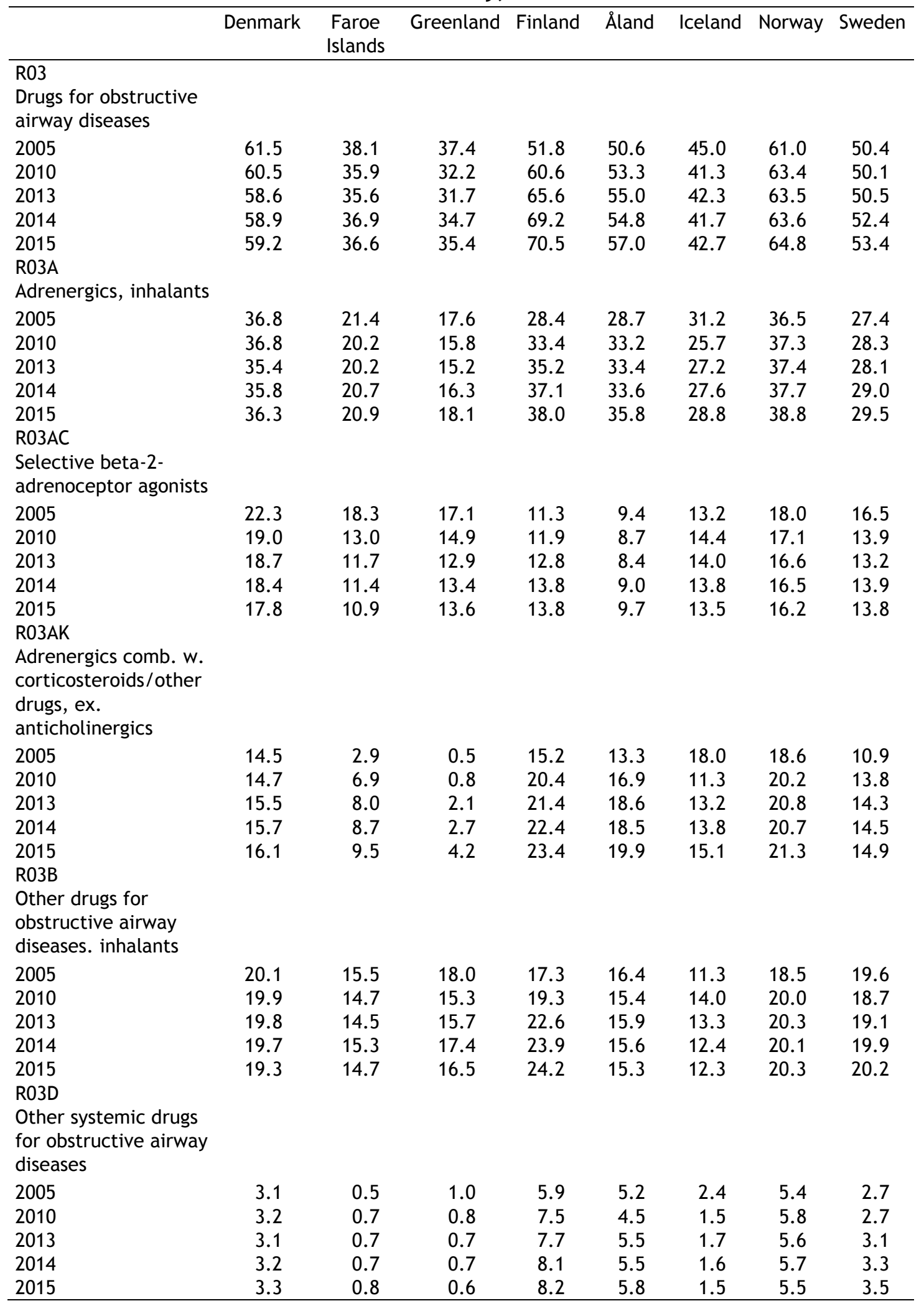


Figure 3.7.11 Sales of drugs for obstructive airway diseases (ATC-Group R03), DDD/1 000 inhabitants/day, 2005-2015

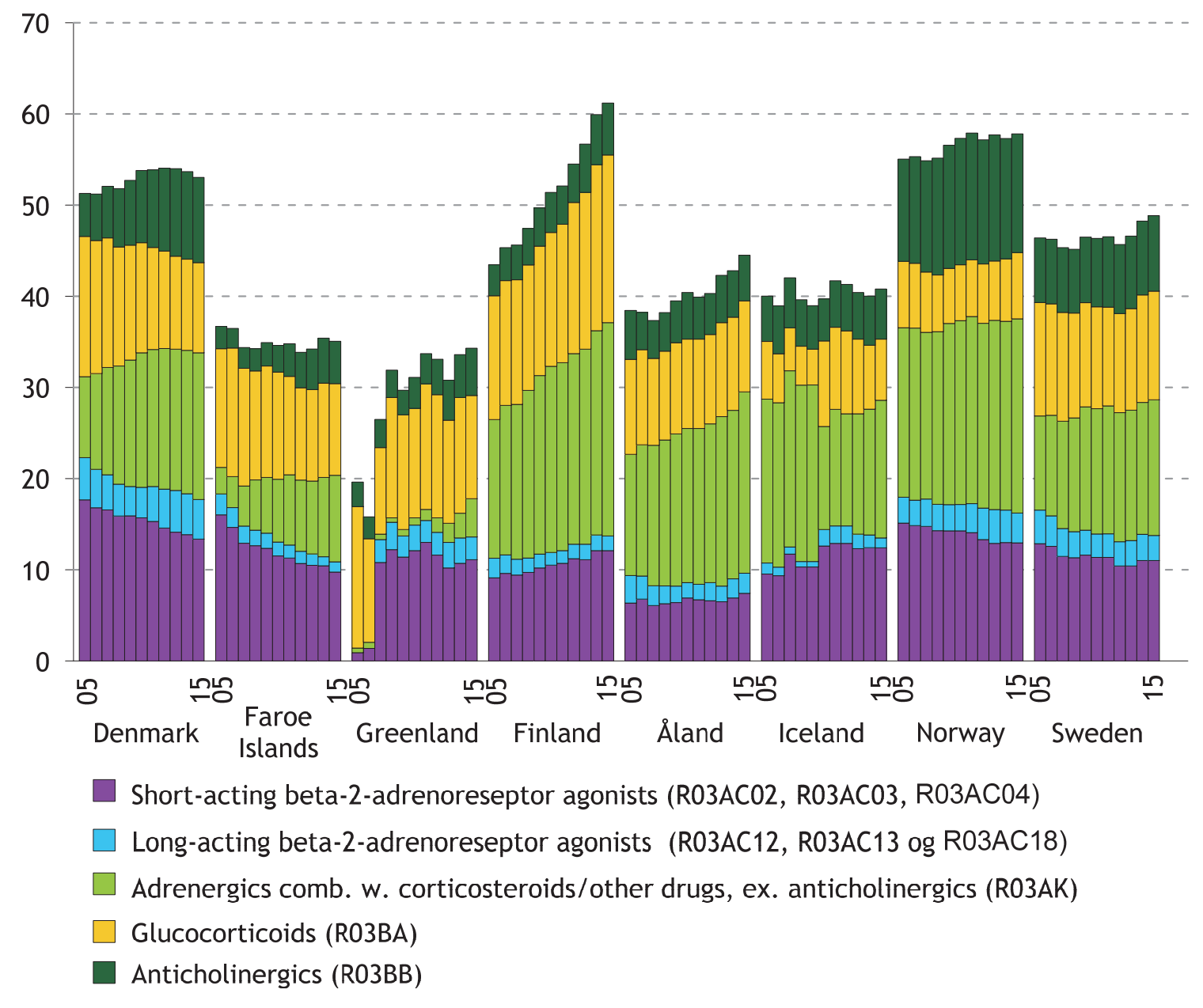


Morbidity, Medical Treatment, Accidents and Pharmaceutical products

Table 3.7.38 Proportion of the population per 1000 by age and gender (oneyear prevalence) receiving at least one inhalant for obstructive airway diseases (ATC-groups R03A and R03B), 2015

\begin{tabular}{|c|c|c|c|}
\hline & Men & Women & Total \\
\hline \multicolumn{4}{|c|}{ Denmark } \\
\hline $0-14$ & 80 & 57 & 69 \\
\hline $15-24$ & 39 & 48 & 43 \\
\hline $25-44$ & 46 & 55 & 50 \\
\hline $45-64$ & 70 & 94 & 82 \\
\hline $65-74$ & 110 & 136 & 123 \\
\hline $75+$ & 162 & 157 & 160 \\
\hline Total & .. & .. & .. \\
\hline \multicolumn{4}{|c|}{ Faroe Islands } \\
\hline $0-14$ & 95 & 65 & 80 \\
\hline $15-24$ & 49 & 60 & 54 \\
\hline $25-44$ & 45 & 60 & 52 \\
\hline $45-64$ & 51 & 80 & 65 \\
\hline $65-74$ & 93 & 136 & 113 \\
\hline $75+$ & 115 & 119 & 117 \\
\hline Total & 66 & 78 & 72 \\
\hline \multicolumn{4}{|c|}{ Finland } \\
\hline $0-14$ & 103 & 71 & .. \\
\hline $15-24$ & 56 & 76 & .. \\
\hline $25-44$ & 62 & 100 & .. \\
\hline $45-64$ & 90 & 138 & .. \\
\hline $65-74$ & 121 & 151 & .. \\
\hline $75+$ & 159 & 158 & .. \\
\hline Total & .. & .. & .. \\
\hline \multicolumn{4}{|c|}{ Iceland } \\
\hline $0-14$ & 173 & 136 & 155 \\
\hline $15-24$ & 62 & 92 & 76 \\
\hline $25-44$ & 69 & 107 & 87 \\
\hline $45-64$ & 107 & 202 & 165 \\
\hline $65-74$ & 175 & 298 & 278 \\
\hline $75+$ & 186 & 241 & 264 \\
\hline Total & .. & .. & .. \\
\hline \multicolumn{4}{|c|}{ Norway } \\
\hline $0-14$ & 84 & 60 & 72 \\
\hline $15-24$ & 43 & 57 & 50 \\
\hline $25-44$ & 44 & 61 & 52 \\
\hline $45-64$ & 75 & 108 & 91 \\
\hline $65-74$ & 126 & 158 & 142 \\
\hline $75+$ & 146 & 135 & 139 \\
\hline Total & 73 & 89 & 81 \\
\hline \multicolumn{4}{|c|}{ Sweden } \\
\hline $0-14$ & 83 & 59 & 71 \\
\hline $15-24$ & 43 & 56 & 49 \\
\hline $25-44$ & 44 & 64 & 54 \\
\hline $45-64$ & 66 & 102 & 84 \\
\hline $65-74$ & 98 & 144 & 121 \\
\hline $75+$ & 134 & 147 & 141 \\
\hline Total & 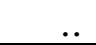 & & \\
\hline
\end{tabular}


Morbidity, Medical Treatment, Accidents and Pharmaceutical products

Table 3.7.39 Sales of antihistamines (ATC-group R06A), DDD/1 000 inhabitants/day, 2005-2015

\begin{tabular}{lcccccccc}
\hline & Denmark & $\begin{array}{c}\text { Faroe } \\
\text { Islands }\end{array}$ & Greenland & Finland & Åland & Iceland & Norway & Sweden \\
\hline 2005 & 20.4 & 20.7 & 7.5 & 31.2 & 24.8 & 30.0 & 54.8 & 30.8 \\
2010 & 27.0 & 25.2 & 10.6 & 42.6 & 31.4 & 38.4 & 58.8 & 36.8 \\
2014 & 35.8 & 35.4 & 17.7 & 54.0 & 37.6 & 50.4 & 68.2 & 48.1 \\
2015 & 34.8 & 31.4 & 13.8 & 51.2 & 37.2 & 53.2 & 67.9 & 48.0 \\
\hline
\end{tabular}


Morbidity, Medical Treatment, Accidents and Pharmaceutical products 


\section{Chapter 4}

\section{Mortality and Causes of Death}

\section{Extra material}

\section{Nowbase.org - Background tables for Health Statistics}

\section{Coding practice and comparability}

Differences in national coding practices are an important factor for comparability between countries of causes of death.

What is shown in the statistics is the underlying cause of death. WHO has drawn up guidelines for the choice of underlying cause of death, i.e. the disease or injury that initiated the chain of morbid events leading directly to death, or the circumstances of the accident or violence that produced the fatal injury. The problem for comparability in some cases is that, where two or more causes of death have been recorded on the death certificate, the choice of the underlying cause of death will differ from country to country, since the ICD rules can be interpreted differently.

Apart from the fact that the ICD rules governing mortality coding give room for interpretation, different national traditions for the choice of underlying cause of death may also develop. An example of this is the use of the diagnostic group "insufficiently defined conditions" (codes 146.9, 195.9, 19.9; J96.0, J96.9, P285.0, R00.0-R94. and R96-99). The use of these codes as underlying cause of death is more widespread in Denmark than in the other Nordic countries in situations where more specific causes of death are also recorded on the death certificate (See Table 4.1.11).

Several other factors also influence comparability, such as the type of information the statistician has access to and the quality of the material (death certificates, etc.).

In order to aid the choice of underlying cause of death, the American programme ACME (Automated Classification of Medical Entities) has been developed. This system is used in most of the Nordic countries. Denmark has used ACME from the data year 2002, Iceland has used ACME for a few years to check manual coding, and Norway and Finland have used ACME from the data year 2005. Otherwise, computer-aided coding has been used. Automatic coding does not necessarily result in a more correct picture of the pattern of causes of death than manual coding, but it does give more consistency in the coding and thus contributes to better comparability between more countries. 
Figure 4.1.1 National coding compared to ACME 2001-2014

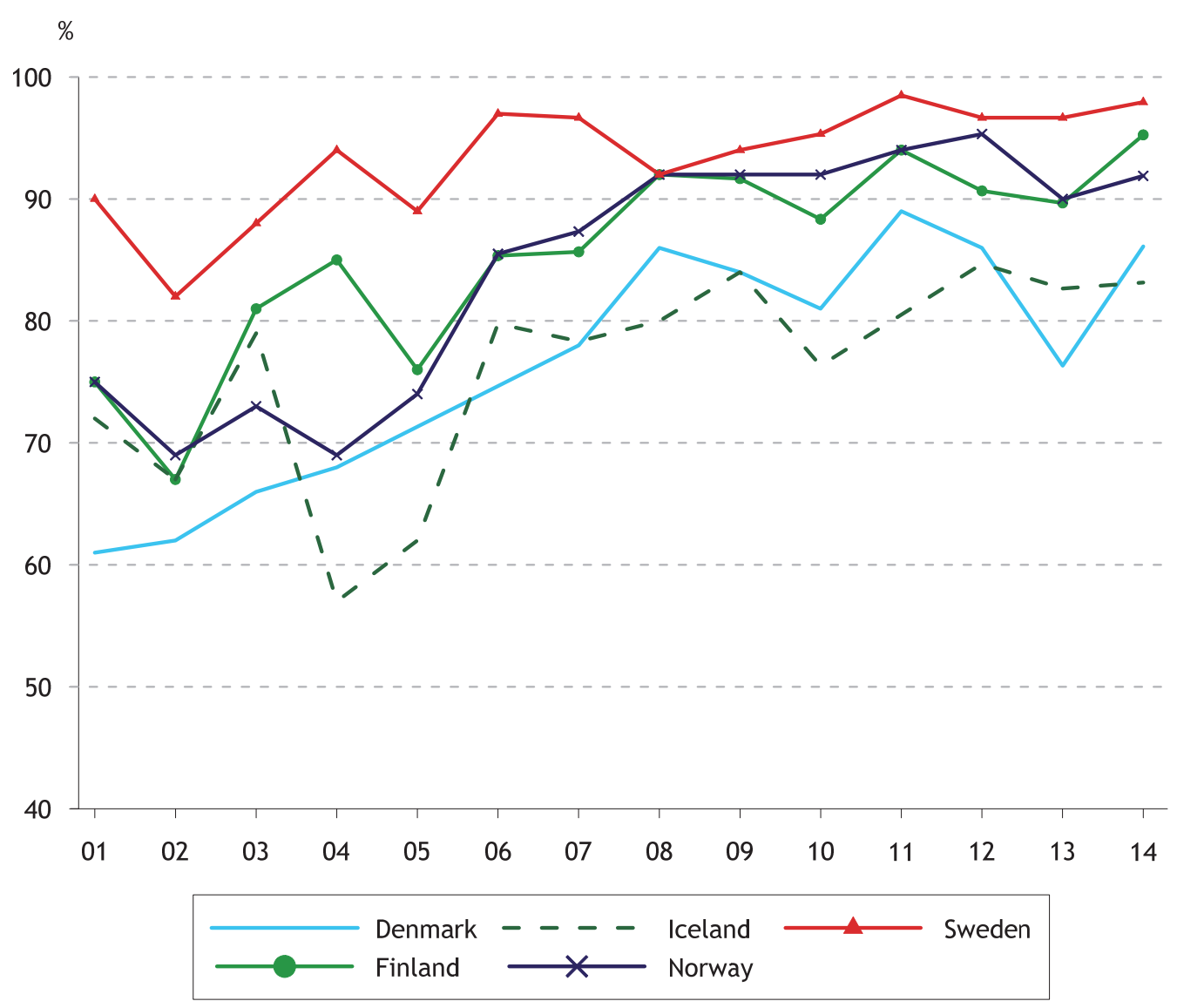

Since 2001, the Nordic Classification Centre has carried out annual comparisons of how the countries classify a sample of causes of death. The sample is relatively small (200-250 death certificates per year), but the results still give an indication of how comparable the statistics are. When making comparisons, the ACME classification system is used as the standard.

This comparison, and Nordic coding practice in general, is discussed at annual meetings. As seen in Figure 4.1.1, the coding in the different countries is not only getting closer to ACME's coding, but the differences in coding between the countries are also getting smaller. This indicates that the use of automatic coding and cooperation between the Nordic countries leads to a higher degree of comparability of mortality statistics.

Cultural differences in the reporting of certain conditions may also influence comparability. For example, if doctors in one country are far more reluctant to register suicide on the death certificate than are doctors in other countries, this can make comparisons difficult. However, in several of the Nordic countries, there are routines for contacting the doctor or the hospital in cases where the external cause of an injury is unclear. Such quality-control practices help to compensate for lack of information on the death certificate. 


\section{Autopsy rates}

Another factor influencing the quality of the statistics on causes of death is decreasing autopsy rates (in 2013, the Danish rate was the lowest at 4 per cent, and the Finnish rate was the highest at 24 per cent). Autopsy rates have been more than halved in the Nordic countries over the last few decades. Studies have shown that in about 30 per cent of cases, the result of the autopsy has caused the underlying cause of death to be altered.

\section{The reliability of the statistics}

Considering the reservations in relation to the comparability of causes of death over time and between countries, the data presented here should be interpreted with caution. This is especially the case for the small diagnostic groups in the European short list that is used in the present publication. The picture is more stable for the large groups, such as cardiovascular diseases and cancer. This also applies to alcohol and drug-related deaths, for which it is well known that the pattern is heterogeneous. The dramatic fall in the number of deaths from AIDS is related to new, lifeprolonging medication. However, there has been a slight increase in the number of new cases in all the Nordic countries. The high incidence of cancer as an underlying cause of death in Denmark is also partly the result of coding practice.

Falls are coded much more often in Denmark than in Sweden. This makes comparison of death statistics for accidents unreliable. The incidence of accidents in total is highest in Finland.

For insufficiently defined conditions, Finland and Iceland are atypical compared with the other Nordic countries, because there are only a few cases of insufficiently defined conditions. 
Table 4.1.1 Deaths by age and gender per 100000 inhabitants, 2000-2014

\begin{tabular}{|c|c|c|c|c|c|c|c|c|c|c|c|c|}
\hline \multirow{2}{*}{$\begin{array}{l}\text { Age } \\
\text { Gender }\end{array}$} & \multicolumn{2}{|c|}{ Total } & \multicolumn{2}{|c|}{$\begin{array}{c}\text { Under } 1 \\
\text { year }^{1}\end{array}$} & \multicolumn{2}{|c|}{ 1-14 years } & \multicolumn{2}{|c|}{$15-24$ years } & \multicolumn{2}{|c|}{ 25-64 years } & \multicolumn{2}{|c|}{$65+$ years } \\
\hline & M & W & M & W & $M$ & W & $M$ & W & $M$ & W & M & W \\
\hline \multicolumn{13}{|c|}{ Denmark } \\
\hline 2000 & 1069 & 1099 & 607 & 456 & 17 & 12 & 79 & 30 & 4414 & 294 & 6368 & 5455 \\
\hline 2010 & 965 & 984 & 363 & 320 & 9 & 7 & 41 & 21 & 408 & 254 & 4936 & 4622 \\
\hline 2013 & 938 & 933 & 332 & 367 & 11 & 7 & 32 & 16 & 367 & 224 & 4463 & 4138 \\
\hline 2014 & 909 & 919 & 455 & 348 & 7 & 6 & 28 & 16 & 351 & 216 & 4239 & 3280 \\
\hline \multicolumn{13}{|l|}{$\begin{array}{l}\text { Faroe } \\
\text { Islands }\end{array}$} \\
\hline 2005-09 & 848 & 767 & 712 & 426 & 38 & 28 & 33 & 19 & 336 & 172 & 5287 & 4308 \\
\hline $2010-14$ & 805 & 750 & 546 & 369 & 12 & 8 & 39 & 46 & 303 & 164 & 4430 & 3857 \\
\hline \multicolumn{13}{|c|}{ Greenland } \\
\hline 2005-09 & 840 & 726 & 1033 & 1200 & 74 & 76 & 344 & 163 & 617 & 473 & 7117 & 6186 \\
\hline $2010-14$ & 831 & 727 & 935 & 561 & 20 & 32 & 348 & 180 & 605 & 389 & 5885 & 6375 \\
\hline \multicolumn{13}{|l|}{ Finland } \\
\hline 2000 & 952 & 954 & 424 & 324 & 14 & 14 & 96 & 34 & 504 & 222 & 5545 & 4606 \\
\hline 2010 & 971 & 929 & 259 & 192 & 12 & 11 & 80 & 27 & 484 & 217 & 4719 & 4047 \\
\hline 2013 & 959 & 935 & 184 & 152 & 10 & 9 & 58 & 26 & 424 & 199 & 4355 & 3842 \\
\hline 2014 & 963 & 956 & 236 & 200 & 11 & 10 & 57 & 21 & 402 & 188 & 4296 & 3871 \\
\hline \multicolumn{13}{|l|}{ Åland } \\
\hline 2005-09 & 938 & 962 & 194 & 152 & 19 & 30 & 41 & 20 & 288 & 159 & 5167 & 4602 \\
\hline $2010-14$ & 954 & 964 & - & - & - & 10 & 59 & 53 & 327 & 151 & 4340 & 4290 \\
\hline \multicolumn{13}{|l|}{ Iceland } \\
\hline 2005-09 & 628 & 622 & 237 & 159 & 13 & 10 & 65 & 21 & 237 & 152 & 4637 & 4160 \\
\hline $2010-14$ & 639 & 625 & 130 & 198 & 12 & 10 & 39 & 24 & 230 & 149 & 4306 & 3932 \\
\hline \multicolumn{13}{|l|}{ Norway } \\
\hline 2000 & 974 & 985 & 427 & 329 & 18 & 15 & 93 & 33 & 339 & 201 & 6052 & 4965 \\
\hline 2010 & 817 & 878 & 277 & 229 & 12 & 9 & 58 & 30 & 293 & 187 & 4922 & 4581 \\
\hline 2013 & 788 & 845 & 258 & 231 & 8 & 8 & 55 & 34 & 271 & 172 & 4522 & 4227 \\
\hline 2014 & 772 & 818 & 264 & 221 & 11 & 6 & 42 & 18 & 256 & 163 & 4345 & 4121 \\
\hline \multicolumn{13}{|l|}{ Sweden } \\
\hline 2000 & 1041 & 1065 & 399 & 281 & 15 & 12 & 59 & 24 & 305 & 200 & 5829 & 4854 \\
\hline 2010 & 941 & 990 & 273 & 242 & 10 & 10 & 50 & 22 & 283 & 180 & 4747 & 4429 \\
\hline 2013 & 913 & 972 & 292 & 253 & 10 & 7 & 48 & 21 & 260 & 169 & 4360 & 4204 \\
\hline 2014 & 897 & 940 & 255 & 179 & 8 & 8 & 50 & 21 & 251 & 165 & 4233 & 4024 \\
\hline
\end{tabular}

1 Per 100000 live births

Source: the national registers for causes of death 
Table 4.1.2a Death rates from malignant neoplasms (cancer) per 100000 men by age, 2000-2014

\begin{tabular}{|c|c|c|c|c|c|c|c|c|c|}
\hline & & Denmark & $\begin{array}{l}\text { Faroe } \\
\text { Islands } \\
1,3,4\end{array}$ & $\begin{array}{c}\text { Greenland } \\
2,3,4\end{array}$ & Finland & Åland $2,3,4$ & $\begin{array}{c}\text { Iceland } \\
2,3,4\end{array}$ & Norway & Sweden \\
\hline \multicolumn{10}{|l|}{ Age } \\
\hline \multirow[t]{4}{*}{$0-14$} & 2000 & 3 & .. & .. & 2 & - & .. & 3 & 3 \\
\hline & 2010 & 1 & - & 6 & 3 & - & 4 & 3 & 2 \\
\hline & 2013 & 1 & - & 6 & 2 & - & 3 & 1 & 2 \\
\hline & 2014 & 2 & - & 3 & 3 & - & 2 & 3 & 3 \\
\hline \multirow[t]{4}{*}{$15-34$} & 2000 & 9 & .. & .. & 6 & 6 & .. & 7 & 8 \\
\hline & 2010 & 5 & 7 & 7 & 6 & 6 & 8 & 5 & 5 \\
\hline & 2013 & 3 & 3 & 9 & 8 & 6 & 7 & 6 & 5 \\
\hline & 2014 & 5 & 6 & 9 & 7 & - & 6 & 4 & 5 \\
\hline \multirow[t]{4}{*}{$35-44$} & 2000 & 33 & .. & . & 22 & 44 & .. & 32 & 20 \\
\hline & 2010 & 23 & 27 & 47 & 19 & 10 & 14 & 16 & 19 \\
\hline & 2013 & 21 & 23 & 46 & 21 & 10 & 29 & 19 & 18 \\
\hline & 2014 & 20 & 18 & 40 & 16 & 11 & 26 & 26 & 18 \\
\hline \multirow[t]{4}{*}{$45-54$} & 2000 & 148 & .. & .. & 107 & 170 & .. & 120 & 97 \\
\hline & 2010 & 110 & 78 & 133 & 84 & 42 & 95 & 77 & 63 \\
\hline & 2013 & 89 & 76 & 177 & 66 & 93 & 78 & 66 & 62 \\
\hline & 2014 & 87 & 92 & 157 & 65 & 111 & 73 & 70 & 61 \\
\hline \multirow[t]{4}{*}{$55-64$} & 2000 & 462 & .. & .. & 320 & 371 & .. & 348 & 294 \\
\hline & 2010 & 385 & 314 & 596 & 316 & 342 & 256 & 300 & 260 \\
\hline & 2013 & 365 & 261 & 563 & 268 & 270 & 252 & 277 & 241 \\
\hline & 2014 & 365 & 259 & 510 & 270 & 274 & 259 & 258 & 233 \\
\hline \multirow[t]{4}{*}{$65-74$} & 2000 & 1189 & .. & .. & 902 & 1001 &.. & 953 & 826 \\
\hline & 2010 & 970 & 928 & 1868 & 747 & 940 & 795 & 850 & 678 \\
\hline & 2013 & 873 & 802 & 1417 & 750 & 949 & 730 & 720 & 649 \\
\hline & 2014 & 890 & 761 & 1378 & 706 & 847 & 733 & 703 & 633 \\
\hline \multirow[t]{4}{*}{$75+$} & 2000 & 2440 & .. & .. & 1947 & 2081 & - & 2142 & 1935 \\
\hline & 2010 & 2298 & 2077 & 3109 & 1780 & 1890 & 1921 & 2231 & 1920 \\
\hline & 2013 & 2149 & 2066 & 2642 & 1682 & 2038 & 1992 & 2047 & 1850 \\
\hline & 2014 & 2018 & 2126 & 2498 & 1678 & 1896 & 1972 & 2076 & 1768 \\
\hline \multicolumn{10}{|c|}{$\begin{array}{ll}1 & 2000=1996-2000 \\
2 & 2010=2006-10 \\
3 & 2013=2009-13 \\
4 & 2014=2010-14\end{array}$} \\
\hline \multicolumn{10}{|c|}{ ICD10, C00-C97 } \\
\hline Sour & & & & & & & & & \\
\hline
\end{tabular}


Mortality and Causes of Death

Table 4.1.2b Death rates from malignant neoplasms (cancer) per 100000 women, by age, 2000-2014

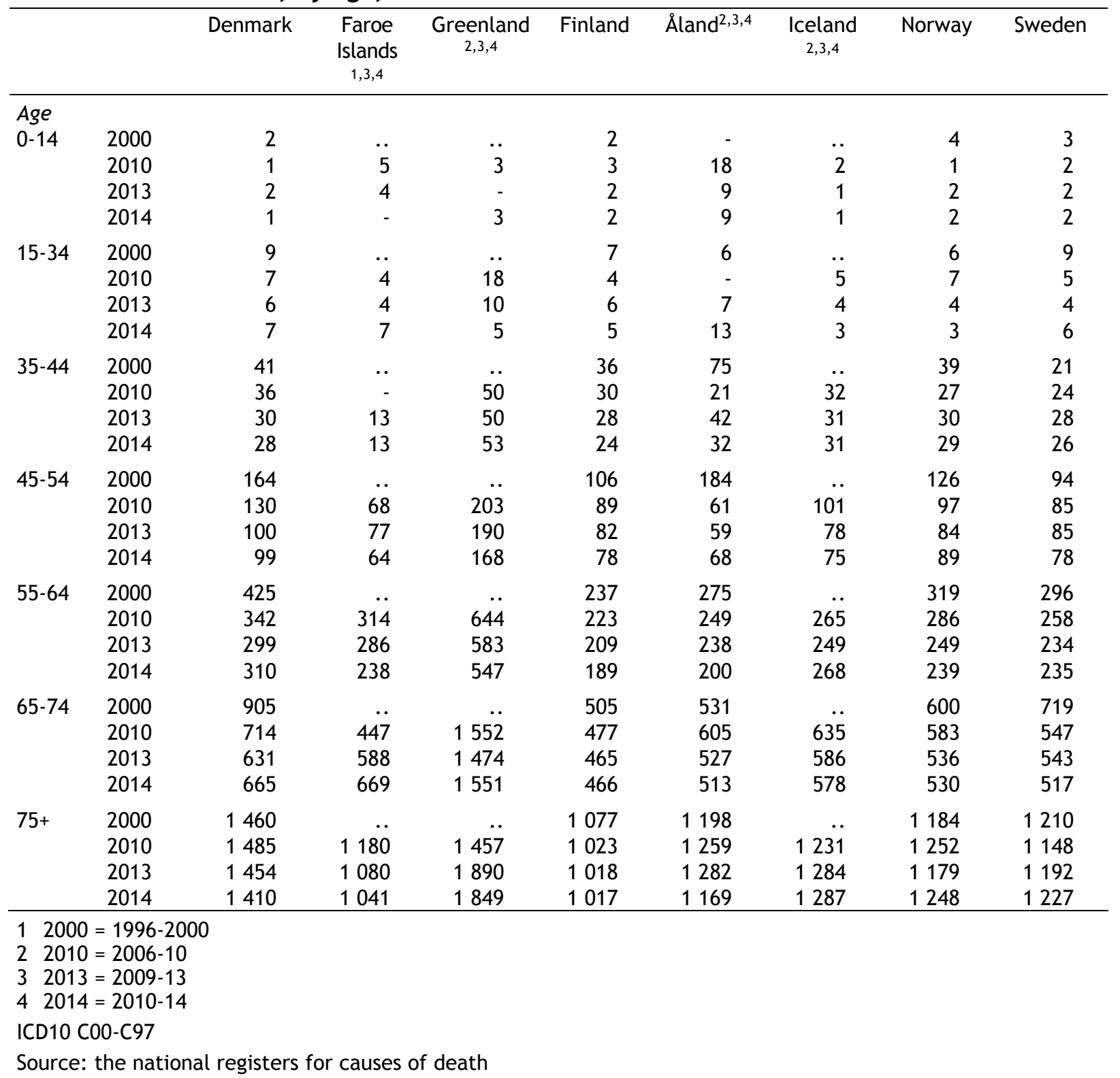


Table 4.1.3a Death rates from circulatory diseases per 100000 men, by age, 2000-2014

\begin{tabular}{|c|c|c|c|c|c|c|c|c|c|}
\hline & & Denmark & $\begin{array}{c}\text { Faroe } \\
\text { Islands } 1,3,4\end{array}$ & $\begin{array}{c}\text { Greenland } \\
2,3,4\end{array}$ & Finland & Åland $2,3,4$ & $\begin{array}{l}\text { Iceland } \\
2,3,4\end{array}$ & Norway & Sweden \\
\hline \multicolumn{10}{|l|}{ Age } \\
\hline \multirow[t]{4}{*}{$0-34$} & 2000 & 3 & .. & 6 & 5 & 7 & .. & 3 & 3 \\
\hline & 2010 & 2 & 2 & 5 & 4 & - & 4 & 2 & 2 \\
\hline & 2013 & 2 & 3 & 4 & 3 & - & 4 & 3 & 3 \\
\hline & 2014 & 1 & 2 & 3 & 3 & - & 3 & 2 & 2 \\
\hline \multirow[t]{4}{*}{$35-44$} & 2000 & 23 & .. & 51 & 44 & 11 & .. & 25 & 21 \\
\hline & 2010 & 22 & 20 & 47 & 28 & 10 & 14 & 23 & 13 \\
\hline & 2013 & 11 & 17 & 51 & 25 & 21 & 11 & 15 & 14 \\
\hline & 2014 & 15 & 18 & 50 & 24 & 11 & 10 & 11 & 14 \\
\hline \multirow[t]{4}{*}{$45-54$} & 2000 & 95 & .. & 179 & 184 & 170 & .. & 93 & 104 \\
\hline & 2010 & 64 & 47 & 88 & 117 & 63 & 66 & 65 & 63 \\
\hline & 2013 & 58 & 64 & 100 & 92 & 62 & 54 & 50 & 62 \\
\hline & 2014 & 58 & 75 & 106 & 91 & 51 & 50 & 48 & 54 \\
\hline \multirow[t]{4}{*}{$55-64$} & 2000 & 326 & .. & 473 & 481 & 445 & .. & 282 & 303 \\
\hline & 2010 & 197 & 216 & 373 & 385 & 171 & 189 & 187 & 217 \\
\hline & 2013 & 262 & 140 & 361 & 323 & 180 & 157 & 152 & 195 \\
\hline & 2014 & 165 & 153 & 409 & 308 & 193 & 157 & 145 & 191 \\
\hline \multirow[t]{4}{*}{$65-74$} & 2000 & 1095 &.. & 1049 & 1378 & 1105 &.. & 1065 & 1101 \\
\hline & 2010 & 557 & 663 & 1552 & 897 & 701 & 643 & 526 & 592 \\
\hline & 2013 & 450 & 544 & 1316 & 770 & 800 & 508 & 445 & 536 \\
\hline & 2014 & 424 & 513 & 1219 & 717 & 719 & 463 & 417 & 510 \\
\hline \multirow[t]{4}{*}{$75+$} & 2000 & 4467 & & 5058 & 4766 & 5051 & $\cdots$ & 4681 & 4851 \\
\hline & 2010 & 2948 & 3654 & 4363 & 3808 & 3939 & 3501 & 3148 & 3946 \\
\hline & 2013 & 2437 & 2993 & 3984 & 3423 & 3418 & 3315 & 2805 & 3476 \\
\hline & 2014 & 2300 & 2811 & 3913 & 3337 & 3462 & 3259 & 2683 & 3148 \\
\hline
\end{tabular}
$12000=1996-2000$
$22010=2006-10$
$32013=2009-13$
$42014=2010-14$
ICD10, 100-199

Source: the national registers for causes of death 
Mortality and Causes of Death

Table 4.1.3b Death rates from circulatory diseases per 100000 women, by age, 2000-2014

\begin{tabular}{|c|c|c|c|c|c|c|c|c|c|}
\hline & & Denmark & $\begin{array}{c}\text { Faroe } \\
\text { Islands }{ }^{1,3,4}\end{array}$ & $\begin{array}{c}\text { Greenland } \\
2,3,4\end{array}$ & Finland & Åland ${ }^{2,3,4}$ & $\begin{array}{c}\text { Iceland } \\
2,3,4\end{array}$ & Norway & Sweden \\
\hline \multicolumn{10}{|l|}{ Age } \\
\hline \multirow[t]{4}{*}{$0-34$} & 2000 & 2 & .. & 7 & 2 & - & .. & 4 & 3 \\
\hline & 2010 & 1 & 5 & - & 3 & 18 & 1 & 1 & 2 \\
\hline & 2013 & 1 & 15 & 7 & 3 & 7 & 1 & 1 & 3 \\
\hline & 2014 & 1 & 6 & 6 & 1 & - & 1 & 1 & 1 \\
\hline \multirow[t]{4}{*}{$35-44$} & 2000 & 14 & .. & 42 & 17 & 11 & .. & 11 & 11 \\
\hline & 2010 & 8 & - & 14 & 9 & - & 6 & 7 & 6 \\
\hline & 2013 & 8 & - & 6 & 8 & - & 8 & 5 & 6 \\
\hline & 2014 & 6 & - & 12 & 8 & - & 8 & 3 & 2 \\
\hline \multirow{4}{*}{$45-54$} & 2000 & 41 & .. & 109 & 48 & 31 & .. & 36 & 34 \\
\hline & 2010 & 25 & 17 & 116 & 31 & 10 & 11 & 21 & 21 \\
\hline & 2013 & 23 & 45 & 63 & 22 & 10 & 15 & 16 & 25 \\
\hline & 2014 & 23 & 45 & 49 & 23 & 20 & 16 & 22 & 19 \\
\hline \multirow[t]{4}{*}{$55-64$} & 2000 & 41 & .. & 271 & 48 & 97 & .. & 36 & 34 \\
\hline & 2010 & 76 & 52 & 262 & 91 & 80 & 58 & 61 & 77 \\
\hline & 2013 & 66 & 22 & 245 & 84 & 76 & 47 & 56 & 73 \\
\hline & 2014 & 68 & 14 & 225 & 85 & 48 & 50 & 48 & 68 \\
\hline \multirow[t]{4}{*}{$65-74$} & 2000 & 561 & .. & 1427 & 551 & 402 & .. & 471 & 469 \\
\hline & 2010 & 273 & 262 & 801 & 297 & 213 & 266 & 236 & 269 \\
\hline & 2013 & 199 & 220 & 645 & 257 & 228 & 207 & 197 & 253 \\
\hline & 2014 & 196 & 203 & 699 & 255 & 203 & 192 & 184 & 242 \\
\hline \multirow[t]{4}{*}{$75+$} & 2000 & 3722 & .. & 8038 & 4090 & 3944 & .. & 3794 & 4059 \\
\hline & 2010 & 2635 & 2492 & 3302 & 3345 & 3492 & 2874 & 2907 & 3537 \\
\hline & 2013 & 2111 & 2069 & 3000 & 3057 & 3620 & 2809 & 2699 & 3243 \\
\hline & 2014 & 2018 & 1971 & 3191 & 3021 & 3758 & 2710 & 2597 & 2962 \\
\hline
\end{tabular}

$12000=1996-2000$

$22010=2006-10$

$32013=2009-13$

$42014=2010-14$

ICD10 I00-199

Source: the national registers for causes of death 
Figure 4.1.2 Deaths per 100000 inhabitants by gender, age standardized rates 2000-2014

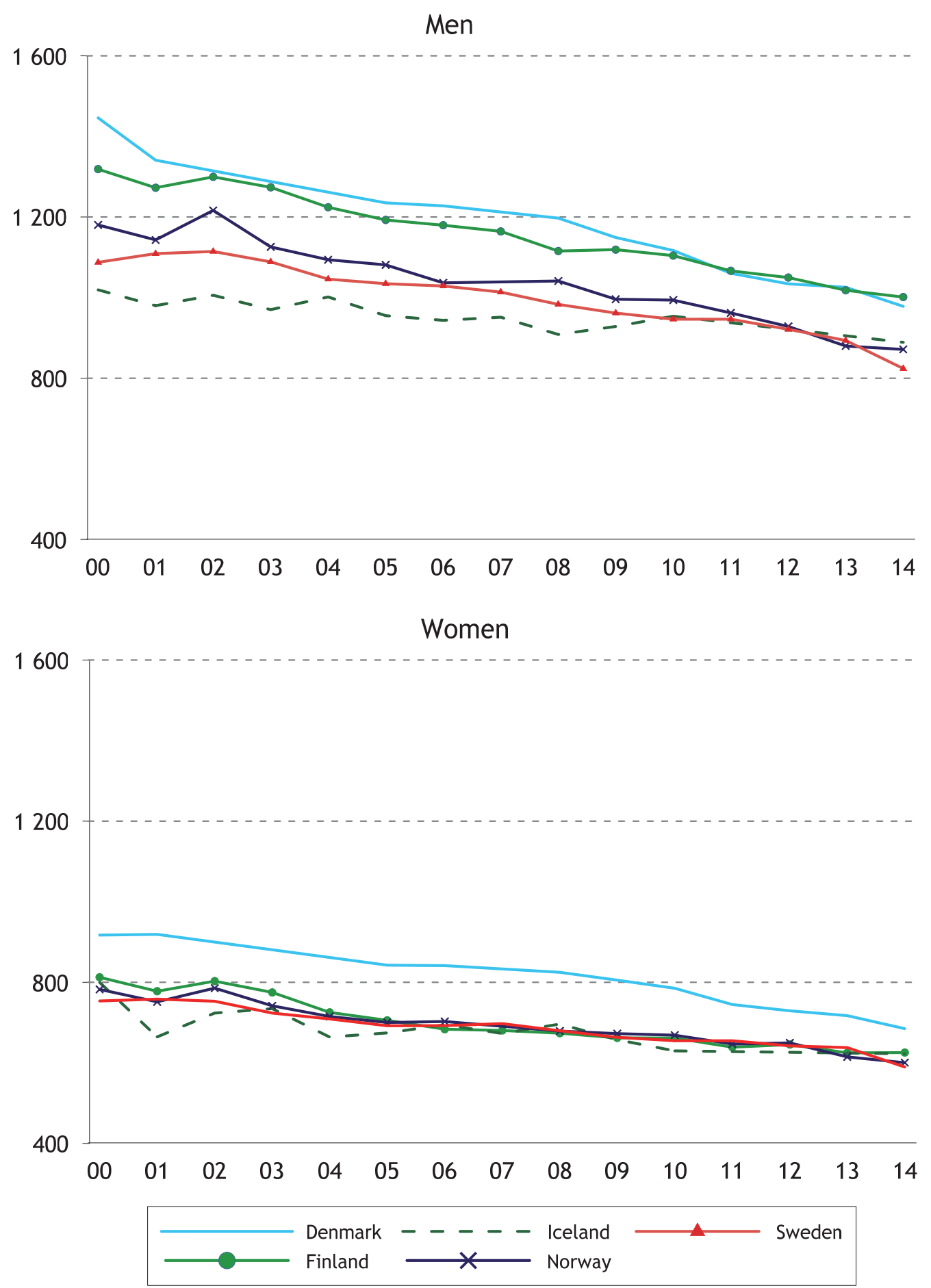

Age-standardized by the Nordic population 2000

Source: the national registers for causes of death 
Figure 4.1.3 Deaths from malignant neoplasms (cancer) per 100000 inhabitants by gender, age standardized rates, 2004-2014
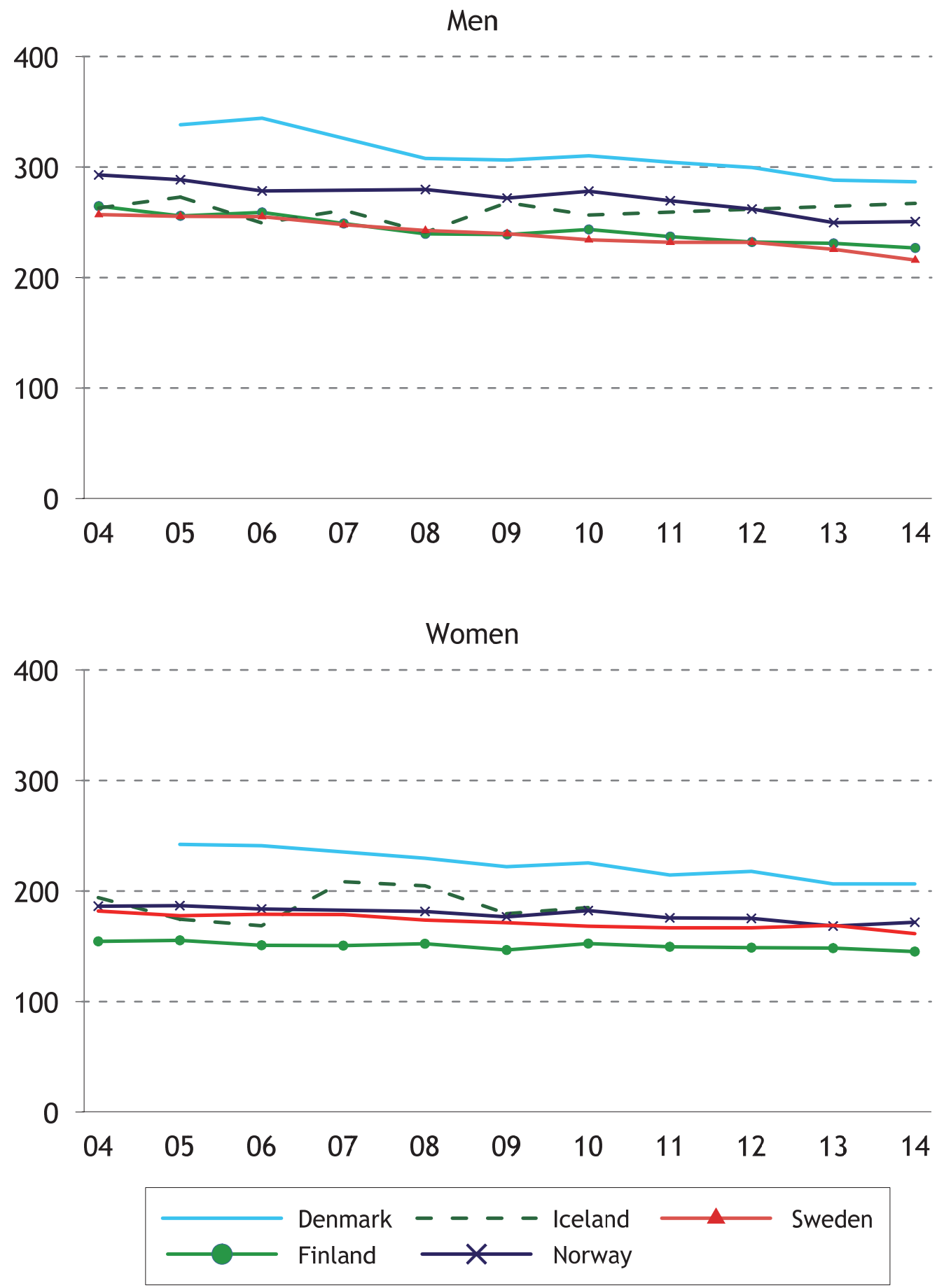

Age-standardized by the Nordic population, 2000 Sources: the national registers for causes of death 
Figure 4.1.4 Deaths from circulatory diseases per 100000 inhabitants by gender, age standardized rates, 2004-2014

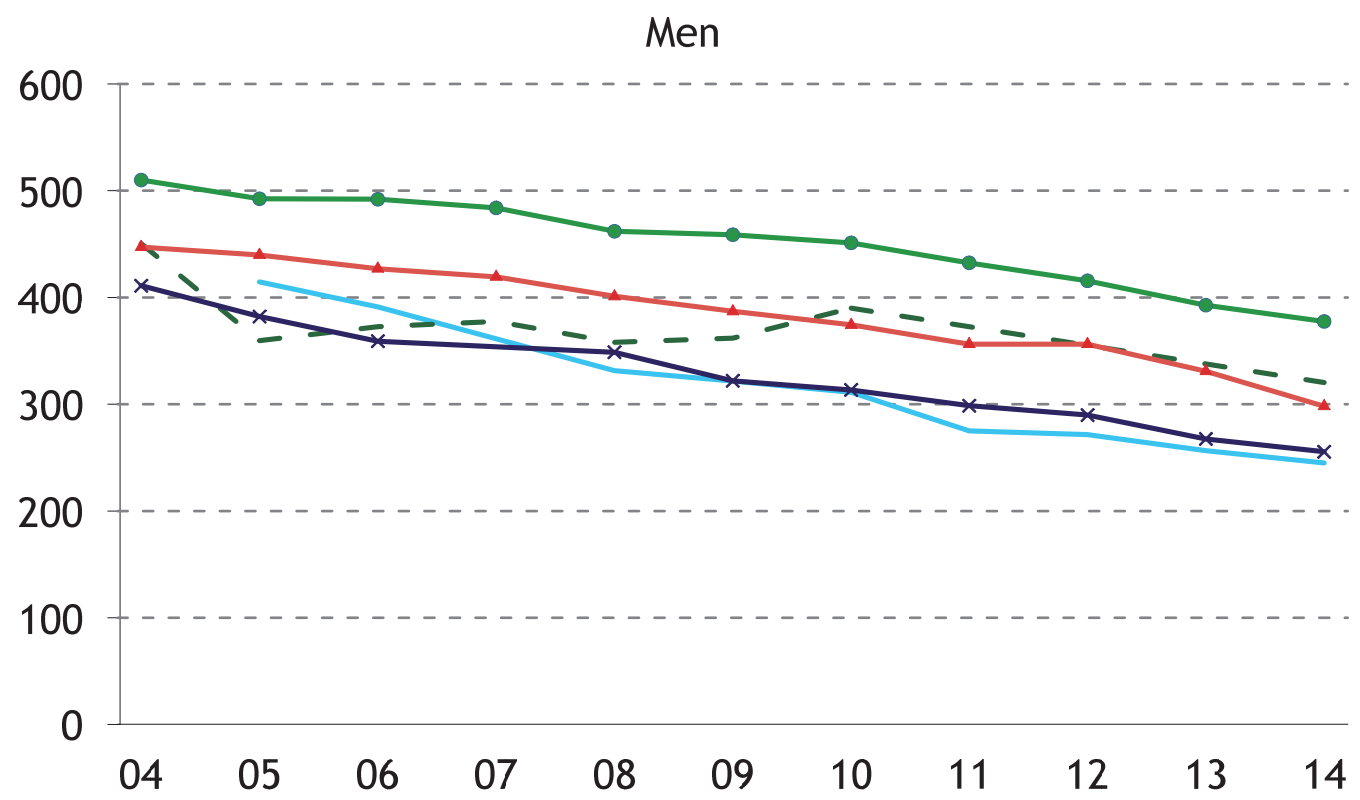

\section{Women}

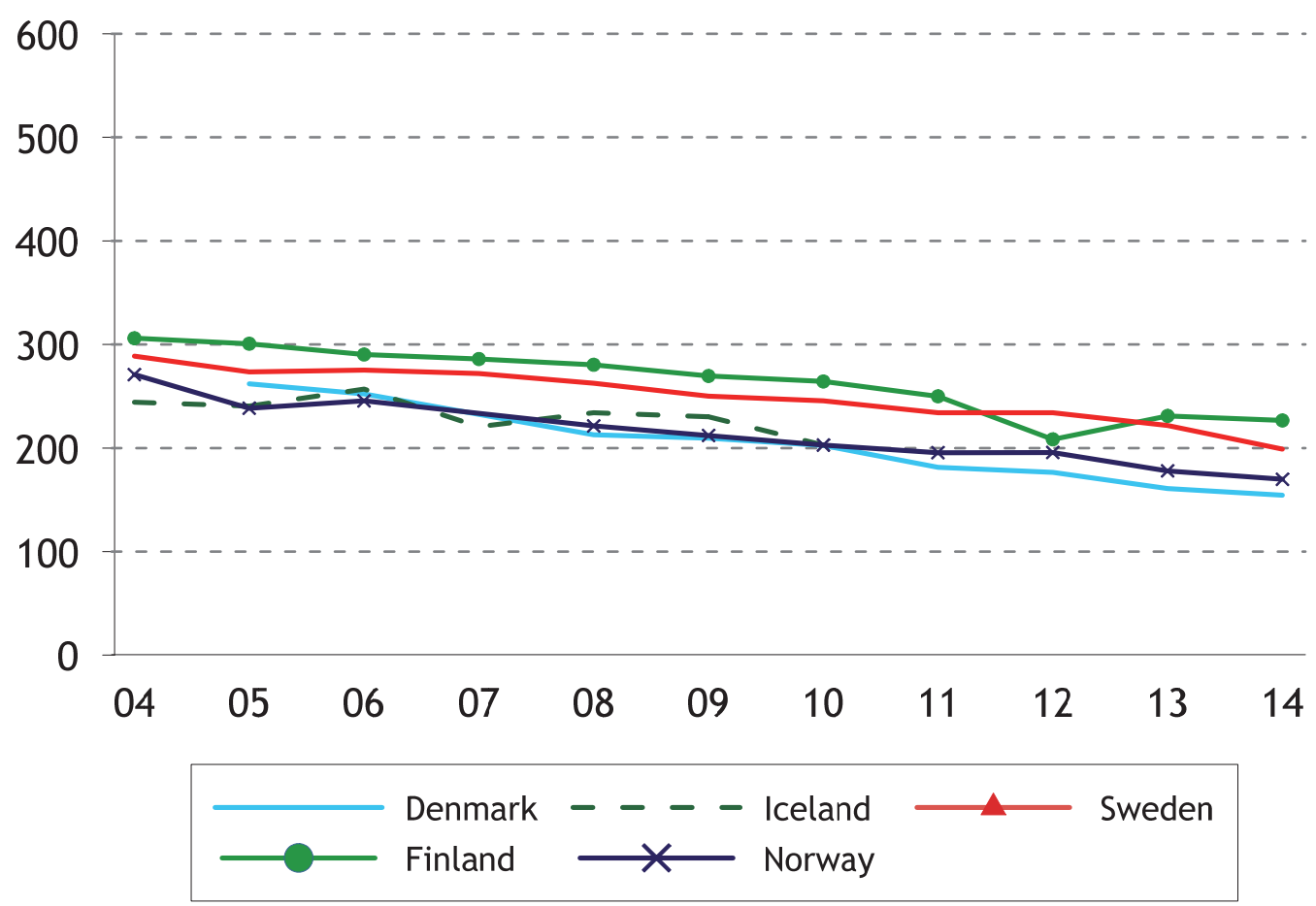

Age-standardized by the Nordic population, 2000

Sources: the national registers for causes of death 
Table 4.1.4 Deaths from avoidable causes per 100000 inhabitants aged 0-74 years

\begin{tabular}{|c|c|c|c|c|c|c|c|c|}
\hline & Denmark & $\begin{array}{l}\text { Faroe } \\
\text { Islands }\end{array}$ & Greenland & Finland & Åland & Iceland & Norway & Sweden \\
\hline ICD-10 code & 2014 & 2010-14 & $2010-14$ & 2014 & $2010-14$ & $2010-14$ & 2014 & 2014 \\
\hline $\begin{array}{l}\text { Malignant neo- } \\
\text { plasm of the } \\
\text { oesophagus (C15) }\end{array}$ & 5.0 & 31.2 & 54.1 & 3.6 & 6.2 & 3.0 & 2.8 & 2.9 \\
\hline $\begin{array}{l}\text { Malignant neo- } \\
\text { plasm of the } \\
\text { trachea, bronchus } \\
\text { and lung (C32-C34) }\end{array}$ & 44.4 & 120.2 & 293.9 & 25.9 & 29.2 & 25.3 & 27.6 & 23.0 \\
\hline $\begin{array}{l}\text { Malignant neo- } \\
\text { plasm of cervix } \\
\text { uteri }{ }^{1} \text { (C53) }\end{array}$ & 2.4 & 18.8 & 42.3 & 1.3 & - & 1.9 & 1.5 & 1.8 \\
\hline Diabetes (E10-E14) & - & 35.6 & 30.7 & 4.6 & 2.3 & 2.2 & 4.2 & 6.1 \\
\hline $\begin{array}{l}\text { Cerebrovascular } \\
\text { diseases } \\
(160-169)\end{array}$ & - & 46.7 & 176.7 & 19.1 & 18.5 & 8.2 & 9.5 & 13.1 \\
\hline $\begin{array}{l}\text { Obstructive lung } \\
\text { diseases } \\
\text { (J40-J44) }\end{array}$ & 19.1 & 40.1 & 66.7 & 8.6 & 12.3 & 6.9 & 12.7 & 9.0 \\
\hline Asthma (J45-J46) & 0.4 & - & 7.2 & 0.3 & 0.8 & 0.0 & 0.3 & 0.2 \\
\hline $\begin{array}{l}\text { Chronic liver dis- } \\
\text { ease and cirrhosis } \\
\text { (K70; K73-K74) }\end{array}$ & 11.9 & 8.9 & 14.4 & 21.6 & 6.9 & 1.1 & 2.7 & 6.4 \\
\hline
\end{tabular}

1 Per 100000 women

Source: the national central statistical bureaus

Table 4.1.5 Deaths from HIV/AIDS in total and per 100000 inhabitants, 20002014

\begin{tabular}{|c|c|c|c|c|c|c|c|c|}
\hline & Denmark & $\begin{array}{c}\text { Faroe } \\
\text { Islands }^{1}\end{array}$ & Greenland $^{1}$ & Finland & Åland ${ }^{1}$ & Iceland $^{1}$ & Norway & Sweden \\
\hline \multicolumn{9}{|c|}{ Number } \\
\hline 2000 & 21 & - & 5 & 10 & - & .. & 15 & 13 \\
\hline 2010 & 29 & - & 2 & 7 & - & 4 & 10 & 11 \\
\hline 2013 & 28 & - & 1 & 4 & - & 3 & 4 & 15 \\
\hline 2014 & 16 & - & 1 & 3 & - & 3 & 11 & 9 \\
\hline \multicolumn{9}{|c|}{$\begin{array}{l}\text { Per } 100000 \\
\text { inhabitants }\end{array}$} \\
\hline 2000 & 0.4 & - & 8.9 & 0.2 & - & .. & 0.3 & 0.1 \\
\hline 2010 & 0.5 & 0.4 & 3.5 & 0.1 & - & 0.2 & 0.2 & 0.1 \\
\hline 2013 & 0.5 & 2.1 & 7.1 & 0.1 & - & 0.2 & 0.1 & 0.2 \\
\hline 2014 & 0.3 & 2.1 & 8.8 & 0.1 & - & 0.2 & 0.2 & 0.1 \\
\hline
\end{tabular}

$12014=2010-14 ; 2013=2009-13 ; 2010=2006-10 ; 2000=1996-2000$

ICD10 B20-B24

Source: the national registers for causes of death 
Figure 4.1.5 Deaths from HIV/AIDS per 100000 inhabitants, 1990-2014

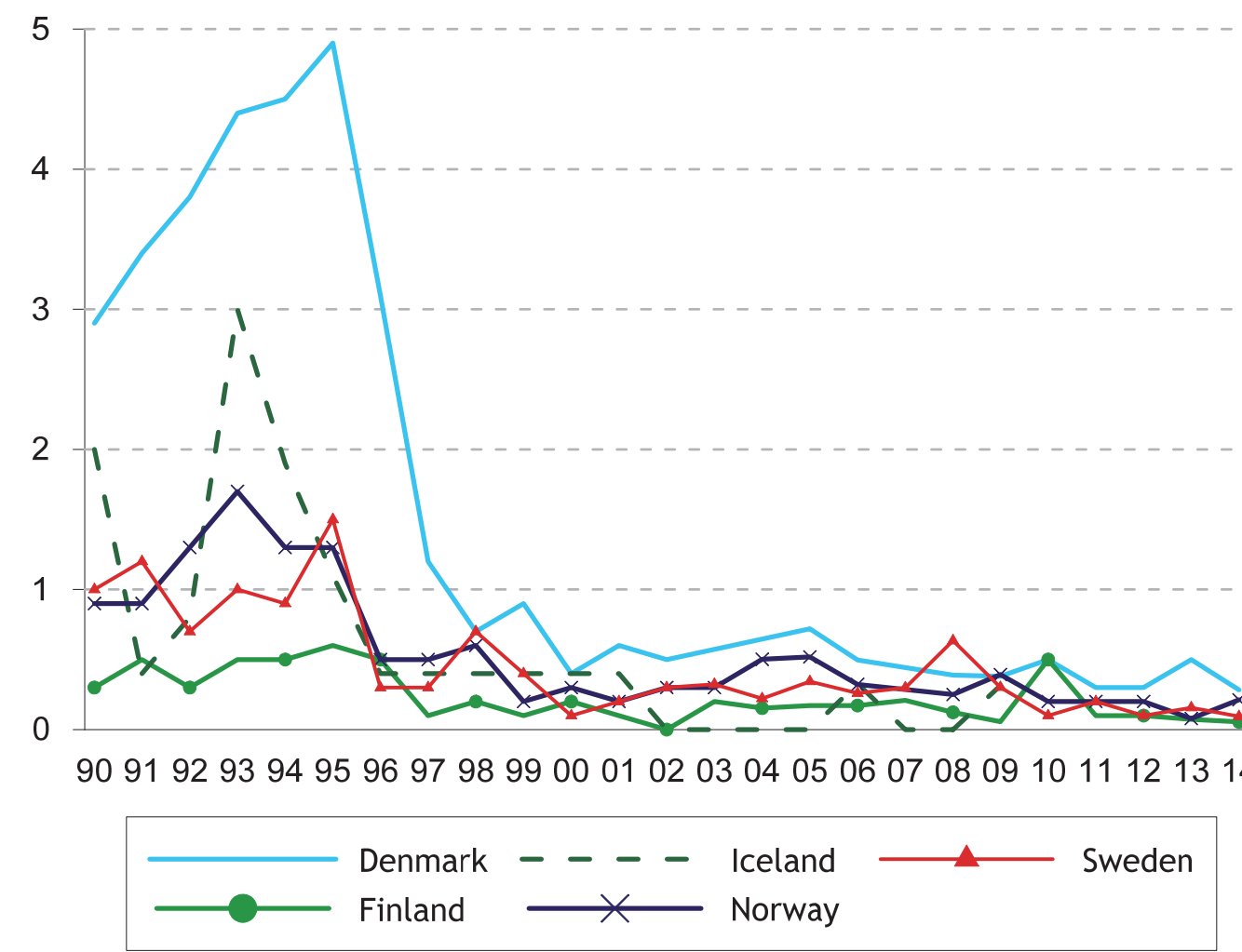

Source: the national registers for causes of death 
Table 4.1.6 Deaths from suicide per 100000 inhabitants by age and gender 19902014

\begin{tabular}{|c|c|c|c|c|c|c|c|c|c|c|c|c|}
\hline & \multicolumn{6}{|c|}{ Men } & \multicolumn{6}{|c|}{ Women } \\
\hline & Total & $10-19$ & $20-24$ & $25-64$ & $65-79^{1}$ & $80+$ & Total & $10-19$ & $20-24$ & $25-64$ & $65-79^{1}$ & $80+$ \\
\hline \multicolumn{13}{|l|}{ Denmark } \\
\hline 1990 & 32.2 & 4.8 & 19.8 & 41.3 & 58.9 & .. & 16.4 & 1.2 & 5.6 & 19.8 & 31.0 & .. \\
\hline 2000 & 20.2 & 4.4 & 16.0 & 23.8 & 34.2 & 70.1 & 7.2 & 2.5 & 1.2 & 8.1 & 10.6 & 23.3 \\
\hline 2010 & 14.7 & 3.4 & 3.0 & 18.5 & 25.4 & 38.1 & 5.7 & 1.2 & 3.7 & 6.8 & 10.4 & 7.5 \\
\hline 2013 & 16.1 & 3.4 & 5.4 & 20.0 & 24.9 & 47.8 & 5.6 & 1.8 & 3.9 & 6.5 & 8.6 & 11.5 \\
\hline 2014 & 16.7 & 4.5 & 8.4 & 21.5 & 9.1 & 36.2 & 5.6 & 0.9 & 2.7 & 6.6 & 9.2 & 15.3 \\
\hline \multicolumn{13}{|l|}{$\begin{array}{l}\text { Faroe } \\
\text { Islands }\end{array}$} \\
\hline 2005-09 & 8.8 & 5.1 & - & 15.5 & - & 0.9 & - & - & 1.8 & - & 8.8 & 5.1 \\
\hline $2010-14$ & 9.6 & - & 11.7 & 14.3 & 14.1 & - & 1.7 & 5.8 & - & - & 6.1 & - \\
\hline \multicolumn{13}{|c|}{ Greenland } \\
\hline 2005-09 & 109.9 & 124.7 & 344.0 & 112.9 & 35.1 & 39.8 & 84.8 & 79.9 & 36.1 & - & 109.9 & 124.7 \\
\hline $2010-14$ & 109.5 & 128.9 & 325.2 & 110.8 & 30.9 & 113.0 & 50.3 & 98.4 & 97.3 & 46.5 & 21.1 & - \\
\hline \multicolumn{13}{|l|}{ Finland } \\
\hline 1990 & 49.4 & 20.6 & 60.3 & 63.9 & 58.0 & 91.5 & 12.5 & 2.6 & 15.8 & 16.7 & 15.2 & 8.6 \\
\hline 2000 & 34.6 & 7.3 & 41.8 & 46.6 & 34.2 & 50.7 & 10.9 & 4.1 & 9.4 & 15.3 & 11.4 & 7.1 \\
\hline 2010 & 27.2 & 9.6 & 44.9 & 33.8 & 24.4 & 37.8 & 8.6 & 2.9 & 13.2 & 11.2 & 8.4 & 7.5 \\
\hline 2013 & 24.9 & 6.8 & 27.6 & 32.0 & 6.5 & 42.5 & 8.0 & 4.4 & 11.4 & 10.3 & 7.9 & 3.3 \\
\hline 2014 & 22.2 & 6.8 & 24.6 & 28.3 & 24.7 & 36.9 & 6.8 & 3.1 & 8.4 & 9.1 & 6.9 & 1.1 \\
\hline \multicolumn{13}{|l|}{ Åland } \\
\hline 2005-09 & 11.9 & - & - & 8.1 & 48.6 & 13.2 & - & 33.0 & 13.5 & 23.2 & 11.9 & - \\
\hline $2010-14$ & 15.5 & - & 24.2 & 21.2 & 10.1 & 37.0 & 4.2 & - & 29.5 & 5.3 & - & - \\
\hline \multicolumn{13}{|l|}{ Iceland } \\
\hline $2005-09$ & 16.8 & 6.8 & 24.6 & 23.9 & 12.7 & 10.3 & 5.8 & - & 1.8 & 9.9 & 5.8 & - \\
\hline $2010-14$ & 18.8 & 8.8 & 19.4 & 24.2 & 26.3 & 38.7 & 6.4 & 1.8 & 6.8 & 8.7 & 9.2 & 5.9 \\
\hline \multicolumn{13}{|l|}{ Norway } \\
\hline 1990 & .. &.. & .. & 33.0 & 33.0 & .. & .. & .. & .. & 10.3 & 11.1 & .. \\
\hline 2000 & 18.4 & 11.3 & 29.9 & 22.5 & 21.0 & 28.0 & 5.8 & 3.0 & 4.4 & 8.0 & 7.8 & 3.1 \\
\hline 2010 & 15.8 & 6.1 & 25.7 & 18.9 & 24.0 & 20.6 & 6.7 & 1.3 & 6.0 & 10.1 & 6.0 & 4.9 \\
\hline 2013 & 14.8 & 5.8 & 14.9 & 19.2 & 19.0 & 18.6 & 7.0 & 1.9 & 8.4 & 10.2 & 5.8 & 1.3 \\
\hline 2014 & 15.5 & 1.8 & 19.3 & 20.6 & 18.9 & 24.6 & 5.8 & 2.6 & 6.0 & 7.9 & 6.2 & 1.3 \\
\hline \multicolumn{13}{|l|}{ Sweden } \\
\hline 1990 & 24.1 & 5.0 & 20.9 & 28.8 & 45.7 &.. & 10.4 & 2.5 & 6.1 & 13.7 & 14.5 & .. \\
\hline 2000 & 18.3 & 4.0 & 15.9 & 21.2 & 33.1 & 45.5 & 7.3 & 3.2 & 3.9 & 9.2 & 9.8 & 3.1 \\
\hline 2010 & 17.9 & 5.6 & 17.7 & 21.9 & 23.1 & 39.9 & 6.4 & 2.6 & 6.3 & 7.9 & 9.3 & 6.7 \\
\hline 2013 & 18.0 & 5.5 & 21.0 & 21.3 & 25.8 & 33.0 & 7.6 & 3.1 & 10.1 & 9.0 & 9.6 & 11.3 \\
\hline 2014 & 16.2 & 5.0 & 18.4 & 19.1 & 20.8 & 36.2 & 7.5 & 3.7 & 6.5 & 9.5 & 8.5 & 10.5 \\
\hline
\end{tabular}

For Faroe Islands, Greenland and Åland 65-80+

ICD10 X60-X84

Source: the national registers for causes of death 
Figure 4.1.6 Deaths from suicide per 100000 inhabitants by gender, age standardized rates, 2000-2014
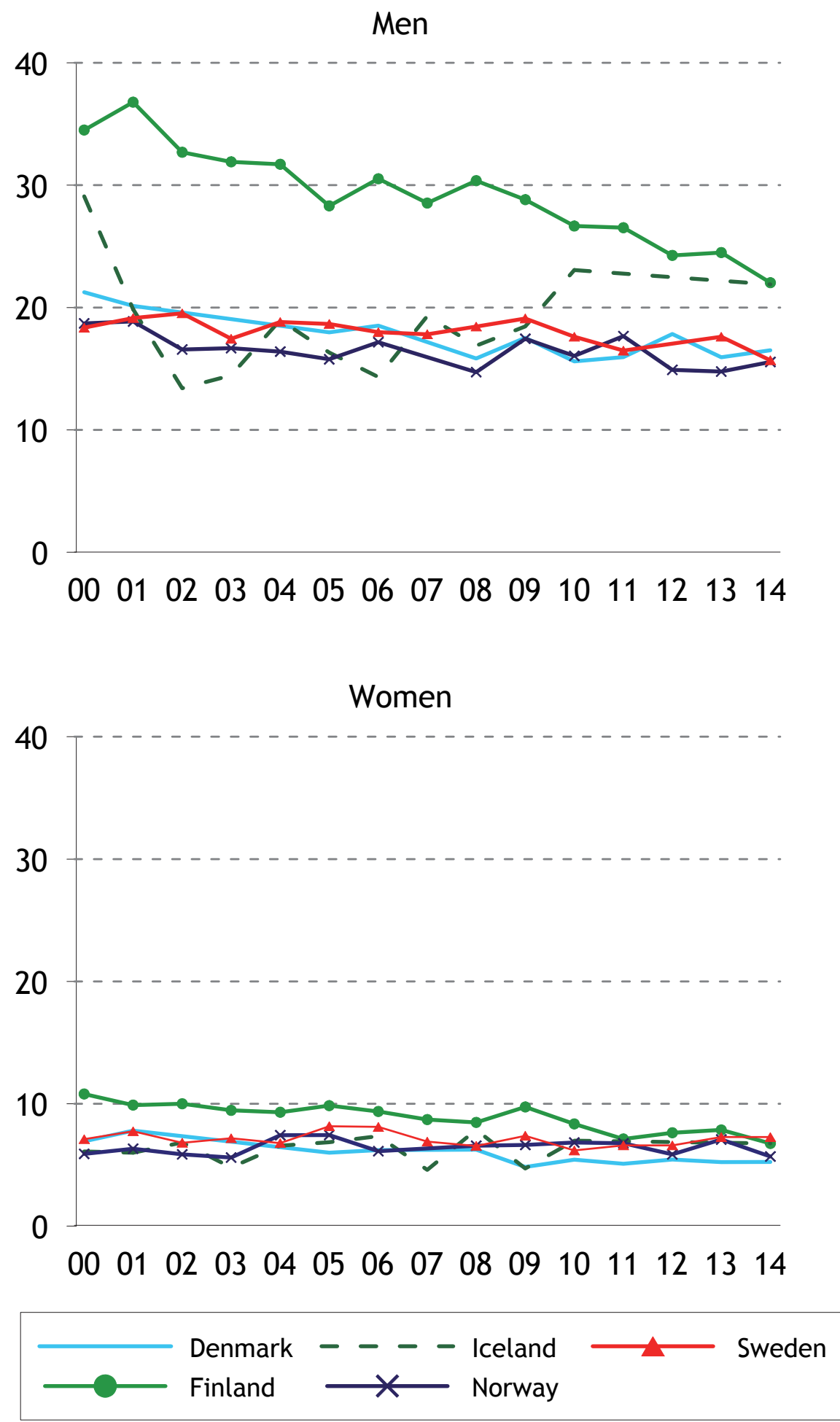

Age-standardized by the Nordic population, 2000

Source: the national registers for causes of death 
Table 4.1.7 Deaths from accidents per 100000 inhabitants by age and gender, 2000-2014

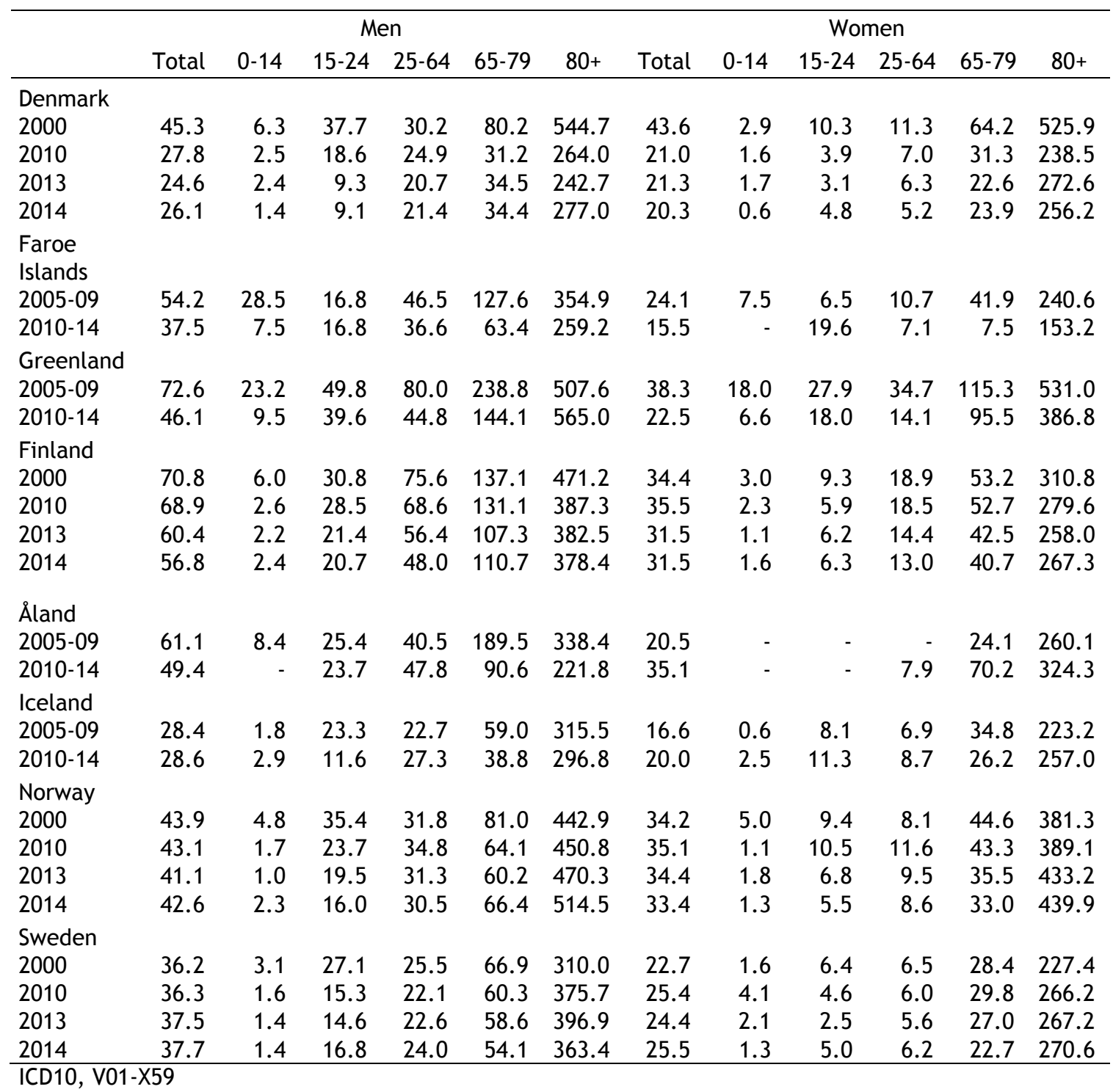

Source: the national registers for causes of death 
Figure 4.1.7 Deaths from accidents per 100000 inhabitants by gender, age standardized rates, 2004-2014
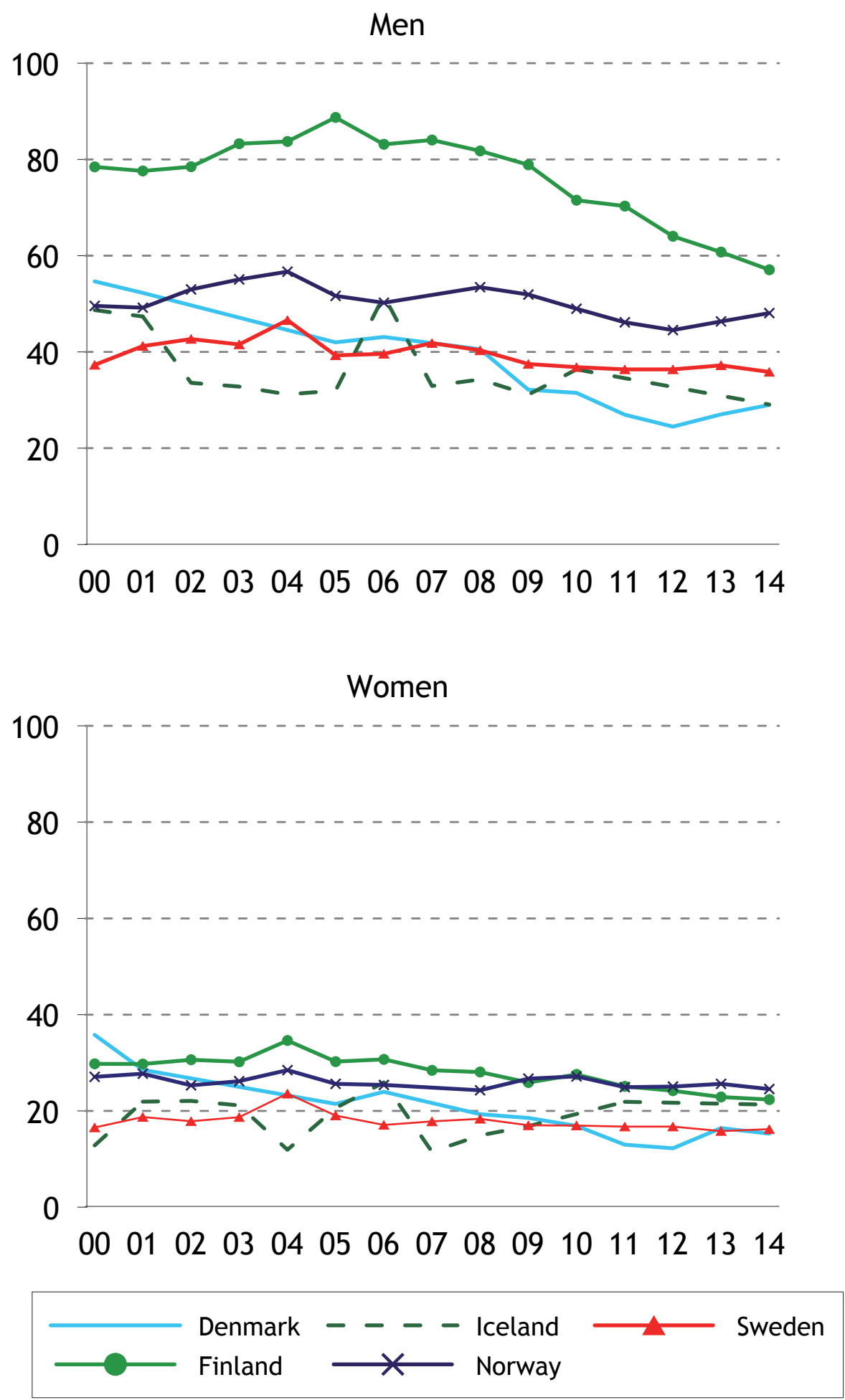

Age-standardized by the Nordic population, 2000

Source: the national registers for causes of death 
Mortality and Causes of Death

Table 4.1.8 Deaths from land transport accidents per 100000 inhabitants by age and gender, 2000-2014

\begin{tabular}{|c|c|c|c|c|c|c|c|c|c|c|c|c|}
\hline & \multicolumn{6}{|c|}{ Men } & \multicolumn{6}{|c|}{ Women } \\
\hline & Total & $0-14$ & $15-24$ & $25-64$ & $65-79^{1}$ & $80+$ & Total & $0-14$ & $15-24$ & $25-64$ & $65-79^{1}$ & $80+$ \\
\hline \multicolumn{13}{|l|}{ Denmark } \\
\hline 2000 & 13.4 & 3.8 & 28.0 & 11.7 & 20.7 & 28.6 & 5.9 & 1.2 & 9.3 & 4.6 & 9.4 & 19.7 \\
\hline 2010 & 7.1 & 1.4 & 10.9 & 7.2 & 6.7 & 24.6 & 3.4 & 1.2 & 3.0 & 2.4 & 7.1 & 12.9 \\
\hline 2013 & 4.7 & 1.0 & 5.4 & 5.1 & 6.2 & 10.5 & 2.4 & 1.3 & 2.9 & 1.5 & 5.2 & 7.7 \\
\hline 2014 & 5.5 & 0.8 & 4.8 & 5.9 & 7.0 & 20.4 & 1.7 & 0.4 & 1.7 & 1.1 & 3.8 & 4.7 \\
\hline \multicolumn{13}{|l|}{$\begin{array}{l}\text { Faroe } \\
\text { Islands }\end{array}$} \\
\hline 2005-09 & 10.4 & 7.1 & 11.2 & 9.3 & 19.8 & 6.0 & 3.8 & 6.5 & 3.6 & 16.9 & 10.4 & 7.1 \\
\hline $2010-14$ & 8.0 & - & 11.2 & 9.6 & 14.1 & - & 2.6 & - & 19.6 & - & - & - \\
\hline \multicolumn{13}{|c|}{ Greenland } \\
\hline 2005-09 & 18.7 & 14.5 & 22.6 & 18.8 & 23.4 & 9.8 & 9.0 & 18.6 & 7.2 & 11.2 & 18.7 & 14.5 \\
\hline $2010-14$ & 2.7 & 3.2 & - & 1.2 & - & 226.0 & 0.8 & - & - & 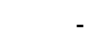 & 11.9 & - \\
\hline \multicolumn{13}{|l|}{ Finland } \\
\hline 2000 & 11.3 & 2.3 & 13.3 & 11.4 & 22.1 & 54.9 & 5.1 & 2.2 & 5.6 & 4.1 & 9.4 & 14.1 \\
\hline 2010 & 8.2 & 0.7 & 14.0 & 7.8 & 13.1 & 16.4 & 2.7 & 1.4 & 2.8 & 2.2 & 5.1 & 4.6 \\
\hline 2013 & 7.4 & 1.1 & 9.8 & 7.4 & 9.9 & 20.1 & 2.7 & 0.5 & 3.1 & 1.6 & 5.7 & 7.7 \\
\hline 2014 & 6.6 & 0.9 & 9.3 & 6.3 & 10.6 & 14.1 & 2.3 & 1.1 & 2.8 & 1.8 & 3.0 & 6.0 \\
\hline \multicolumn{13}{|l|}{ Åland } \\
\hline 2005-09 & 13.4 & 8.4 & 12.7 & 8.1 & 38.9 & 2.9 & - & - & - & 15.5 & 13.4 & 8.4 \\
\hline $2010-14$ & 8.5 & - & 23.7 & 10.6 & - & - & 5.6 & - & - & 2.6 & 30.1 & - \\
\hline \multicolumn{13}{|l|}{ Iceland } \\
\hline 2005-09 & 8.9 & 0.6 & 15.5 & 8.6 & 15.9 & 25.9 & 3.0 & 0.6 & 6.3 & 2.5 & 5.8 & 3.4 \\
\hline $2010-14$ & 4.6 & 1.2 & 6.6 & 5.0 & 4.2 & 12.9 & 2.2 & 2.5 & 4.4 & 0.7 & 5.2 & 5.9 \\
\hline \multicolumn{13}{|l|}{ Norway } \\
\hline 2000 & 14.5 & 2.6 & 27.8 & 15.0 & 16.0 & 28.0 & 4.9 & 2.5 & 8.7 & 3.6 & 9.3 & 7.0 \\
\hline 2010 & 6.9 & 0.4 & 11.2 & 6.8 & 9.7 & 20.6 & 2.2 & 0.2 & 4.6 & 1.9 & 2.6 & 4.9 \\
\hline 2013 & 7.0 & 0.8 & 9.3 & 7.6 & 9.0 & 16.1 & 2.1 & - & 3.1 & 1.8 & 3.3 & 9.0 \\
\hline 2014 & 5.0 & 0.8 & 4.6 & 4.7 & 9.3 & 19.7 & 1.4 & 0.4 & 2.2 & 1.2 & 1.9 & 2.9 \\
\hline \multicolumn{13}{|l|}{ Sweden } \\
\hline 2000 & 10.8 & 1.4 & 19.1 & 10.7 & 15.4 & 20.5 & 3.2 & 1.0 & 4.2 & 2.6 & 5.7 & 14.6 \\
\hline 2010 & 4.4 & 0.9 & 6.6 & 4.5 & 4.8 & 8.7 & 1.7 & 0.5 & 2.5 & 1.3 & 2.8 & 3.5 \\
\hline 2013 & 4.4 & 0.6 & 5.6 & 4.5 & 6.7 & 7.5 & 1.3 & 0.1 & 1.3 & 1.3 & 2.5 & 1.2 \\
\hline 2014 & 4.4 & 0.6 & 3.9 & 4.1 & 7.8 & 13.4 & 1.8 & 0.6 & 1.9 & 1.5 & 1.9 & 5.9 \\
\hline
\end{tabular}

1 For Faroe Islands and Greenland 65-80+

ICD10, V01-V89

Source: the national registers for causes of death 
Figure 4.1.8 Deaths from land transport accidents per 100000 inhabitants by gender, 2000-2014
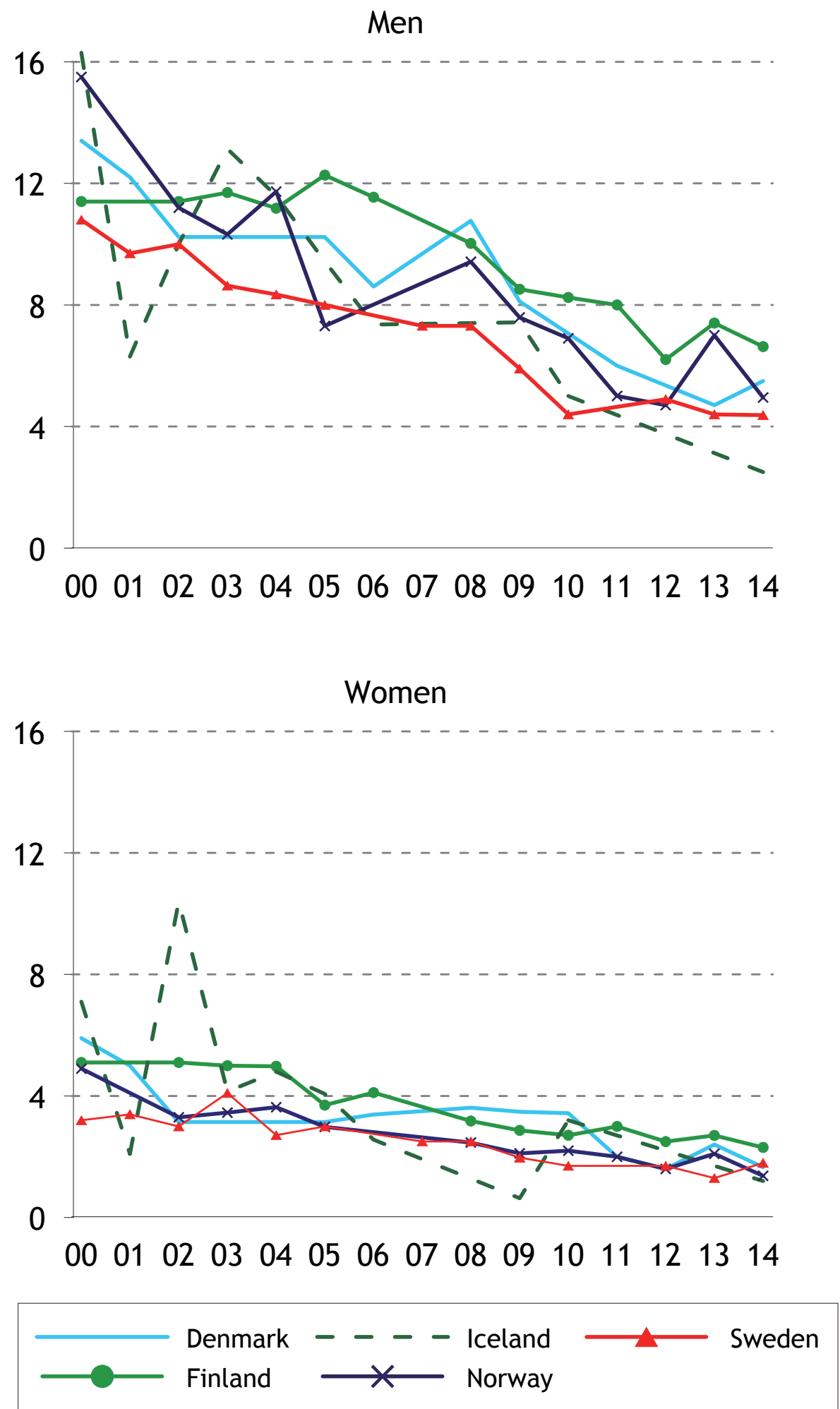

Source: the national registers for causes of death 
Mortality and Causes of Death

Table 4.1.9 Deaths from alcohol-related causes per 100000 inhabitants by age and gender

\begin{tabular}{|c|c|c|c|c|c|c|c|c|}
\hline & Denmark & $\begin{array}{l}\text { Faroe } \\
\text { Islands }\end{array}$ & Greenland & Finland & Åland & Iceland & Norway & Sweden \\
\hline & 2014 & $2010-14$ & $2010-14$ & 2014 & $2010-14$ & 2010-14 & 2014 & 2014 \\
\hline \multicolumn{9}{|l|}{ Men } \\
\hline $0-34$ & 0.5 & - & - & 2.4 & - & 0.5 & - & 0.2 \\
\hline $35-44$ & 10.1 & 59.5 & 24.8 & 24.9 & - & 2.8 & 1.4 & 3.0 \\
\hline $45-64$ & 73.2 & 139.1 & 191.8 & 117.3 & 55.7 & 10.1 & 19.1 & 23.3 \\
\hline $65-74$ & 98.8 & 229.1 & 792.6 & 110.5 & 38.5 & 16.5 & 32.8 & 39.1 \\
\hline $75+$ & 55.2 & 209.8 & 832.6 & 55.7 & 20.6 & 9.7 & 23.8 & 19.4 \\
\hline Total & 35.2 & 75.7 & 116.8 & 51.9 & 21.2 & 4.7 & 9.3 & 12.2 \\
\hline \multicolumn{9}{|l|}{ Women } \\
\hline $0-34$ & 0.2 & - & - & 0.1 & - & - & 0.1 & - \\
\hline $35-44$ & 2.9 & - & 29.7 & 3.3 & - & 1.9 & 0.9 & 0.7 \\
\hline $45-64$ & 24.9 & 16.9 & 57.1 & 30.2 & 14.5 & 3.1 & 6.7 & 9.5 \\
\hline $65-74$ & 38.4 & 101.4 & 380.1 & 53.4 & 27.0 & 12.5 & 8.0 & 11.8 \\
\hline $75+$ & 17.7 & 50.0 & 298.2 & 19.1 & - & 1.8 & 6.1 & 3.8 \\
\hline Total & 12.8 & 17.2 & 45.0 & 14.6 & 7.0 & 2.0 & 3.1 & 4.2 \\
\hline \multicolumn{9}{|l|}{$M+W$} \\
\hline $0-34$ & 0.3 & - & - & 1.2 & - & 0.3 & - & 0.1 \\
\hline $35-44$ & 6.5 & 31.4 & 27.0 & 12.9 & - & 2.4 & 1.1 & 1.8 \\
\hline $45-64$ & 49.1 & 80.8 & 132.4 & 81.1 & 34.6 & 6.6 & 13.0 & 16.5 \\
\hline $65-74$ & 67.7 & 168.5 & 609.1 & 84.4 & 32.9 & 14.5 & 20.2 & 25.2 \\
\hline $75+$ & 33.1 & 116.9 & 521.8 & 37.1 & 8.3 & 5.2 & 13.1 & 10.3 \\
\hline Total & 23.9 & 47.6 & 83.0 & 32.9 & 14.1 & 3.4 & 6.2 & 8.2 \\
\hline
\end{tabular}

Source: the national registers for causes of death

ICD-10: E244, F10, G312, G621, G721, 1426, K292, K700-709, K860, O354, P043, Q860, Y15, X45 
Table 4.1.10 Deaths from drug-related causes per 100000 inhabitants by age and gender

\begin{tabular}{|c|c|c|c|c|c|c|c|c|}
\hline & Denmark & $\begin{array}{c}\text { Faroe } \\
\text { Islands }\end{array}$ & Greenland & Finland & Åland & Iceland & Norway & Sweden \\
\hline & 2014 & $2010-14$ & $2010-14$ & 2014 & $2010-14$ & $2010-14$ & 2014 & 2014 \\
\hline \multicolumn{9}{|l|}{ Men } \\
\hline $0-34$ & 2.7 & - & 6.7 & 2.4 & - & 2.2 & 8.2 & 7.1 \\
\hline $35-44$ & 10.9 & - & - & 3.6 & - & 4.7 & 19.2 & 8.8 \\
\hline $45-64$ & 6.7 & 46.4 & 22.6 & 1.3 & - & 2.5 & 21.0 & 7.8 \\
\hline $65-74$ & 0.7 & - & - & 0.4 & - & 3.7 & 5.7 & 1.7 \\
\hline $75+$ & 1.2 & - & - & 0.6 & - & 2.4 & 5.6 & 1.1 \\
\hline Total & 4.6 & 12.0 & 10.1 & 1.9 & - & 2.7 & 12.7 & 6.5 \\
\hline \multicolumn{9}{|c|}{ Women } \\
\hline $0-34$ & 1.4 & - & - & 0.5 & - & 1.8 & 2.1 & 1.8 \\
\hline $35-44$ & 2.7 & - & - & 1.2 & - & 2.9 & 6.6 & 2.9 \\
\hline $45-64$ & 2.7 & - & - & 1.2 & - & 2.5 & 11.3 & 3.6 \\
\hline $65-74$ & 1.3 & - & - & 0.8 & - & 1.8 & 8.0 & 2.5 \\
\hline $75+$ & 1.6 & - & - & - & - & - & 6.5 & 1.0 \\
\hline Total & 1.9 & - & - & 0.8 & - & 2.0 & 6.0 & 2.4 \\
\hline \multicolumn{9}{|l|}{$M+W$} \\
\hline $0-34$ & 2.1 & - & 3.4 & 1.4 & - & 2.0 & 5.2 & 4.5 \\
\hline $35-44$ & 6.8 & - & - & 2.2 & - & 3.8 & 13.1 & 5.9 \\
\hline $45-64$ & 4.7 & 24.2 & 12.6 & 1.4 & - & 2.5 & 16.3 & 5.7 \\
\hline $65-74$ & 1.0 & - & - & 0.9 & - & 2.7 & 6.9 & 2.1 \\
\hline $75+$ & 1.4 & - & - & 0.8 & - & 1.0 & 6.2 & 1.0 \\
\hline Total & 3.2 & 6.2 & 5.3 & 1.3 & - & 2.4 & 9.3 & 4.4 \\
\hline
\end{tabular}

Source: the national registers for causes of death

ICD-10: F11-F16. F18-F19. O35.5. P04.4 or X40-X49. X60-X69. Y10-Y19. T40.0-T40.3. T40.5-T40.9. T43.6 
Table 4.1.11 Deaths from incompletely defined causes on the death certificate per 100000 inhabitants by age and gender

\begin{tabular}{|c|c|c|c|c|c|c|c|c|}
\hline & Denmark & $\begin{array}{l}\text { Faroe } \\
\text { Islands }\end{array}$ & Greenland & Finland & Åland & Iceland & Norway & Sweden \\
\hline & 2014 & 2010-14 & $2010-14$ & 2014 & $2010-14$ & $2010-14$ & 2014 & 2014 \\
\hline \multicolumn{9}{|l|}{ Men } \\
\hline $0-44$ & 0.1 & - & 15.8 & 0.8 & - & 2.1 & 0.1 & 1.6 \\
\hline $45-64$ & 3.7 & 77.3 & 180.5 & 1.9 & - & 11.1 & 3.3 & 9.8 \\
\hline $65-74$ & 14.3 & 45.8 & 609.7 & 2.5 & - & 16.5 & 11.4 & 25.6 \\
\hline $75+$ & 53.5 & 979.0 & 1040.8 & 4.5 & - & 56.1 & 122.3 & 163.0 \\
\hline Total & 5.9 & 79.7 & 113.5 & 1.5 & - & 8.0 & 8.7 & 18.3 \\
\hline \multicolumn{9}{|l|}{ No death } \\
\hline certificate &.. &.. & .. & 308 & 3 & .. & 434 & 430 \\
\hline \multicolumn{9}{|l|}{ Women } \\
\hline $0-44$ & 0.2 & 14.9 & 5.7 & $3-$ & - & 0.2 & - & 0.8 \\
\hline $45-64$ & 1.9 & 50.7 & 14.3 & 1.1 & - & 4.6 & 1.1 & 4.2 \\
\hline $65-74$ & 7.2 & 50.7 & 152.0 & 1.9 & - & 9.0 & 5.9 & 15.1 \\
\hline $75+$ & 81.3 & 650.4 & 745.6 & 7.5 & 27.8 & 70.1 & 204.8 & 247.2 \\
\hline Total & 8.5 & 81.6 & 33.8 & 1.3 & 2.8 & 6.6 & 18.0 & 29.0 \\
\hline \multicolumn{9}{|l|}{ No death } \\
\hline certificate & .. & .. & .. & 169 & 2 & .. & 296 & 474 \\
\hline \multicolumn{9}{|l|}{$M+W$} \\
\hline $0-44$ & 0.1 & 7.0 & 10.9 & 0.4 & - & 1.2 & - & 1.2 \\
\hline $45-64$ & 2.8 & 64.6 & 107.2 & 1.5 & - & 7.9 & 2.2 & 7.0 \\
\hline $65-74$ & 10.7 & 48.2 & 406.1 & 2.2 & - & 12.7 & 8.6 & 20.3 \\
\hline $75+$ & 69.9 & 788.8 & 869.7 & 6.4 & 16.7 & 64.0 & 171.8 & 212.2 \\
\hline Total & 7.2 & 80.7 & 76.0 & 1.4 & 1.4 & 7.3 & 13.3 & 23.7 \\
\hline \multicolumn{9}{|l|}{ No death } \\
\hline certificate & .. & .. & .. & 477 & 5 & .. & 730 & 904 \\
\hline
\end{tabular}

Table 4.1.12 Autopsy rates as a percentage of all deaths, 2000-2014

\begin{tabular}{|c|c|c|c|c|c|c|c|c|}
\hline & Denmark & $\begin{array}{c}\text { Faroe } \\
\text { Islands }\end{array}$ & Greenland & Finland & Åland & Iceland & Norway & Sweden \\
\hline \multicolumn{9}{|c|}{$\begin{array}{l}\text { Medico-legal } \\
\text { autopsies }\end{array}$} \\
\hline 2000 & 2 & 1 & .. & 21 & 9 & 12 & 4 & 5 \\
\hline 2005 & 3 & 1 & 4 & 24 & 7 & 10 & 4 & 6 \\
\hline 2010 & 2 & 3 & 1 & 23 & 13 & 8 & 3 & 7 \\
\hline 2013 & 2 & 3 & 2 & 18 & 10 & 6 & 4 & 6 \\
\hline 2014 & 2 & 2 & 3 & 17 & 9 & 7 & 4 & 6 \\
\hline \multicolumn{9}{|c|}{$\begin{array}{l}\text { Other } \\
\text { autopsies }\end{array}$} \\
\hline 2000 & 7 & .. & .. & 10 & 9 & 7 & 6 & 9 \\
\hline 2005 & 5 & 1 & 1 & 8 & 3 & 5 & 4 & 8 \\
\hline 2010 & 2 & 1 & - & 7 & 6 & 2 & 4 & 6 \\
\hline 2013 & 2 & 3 & 1 & 6 & 3 & 2 & 3 & 6 \\
\hline 2014 & 2 & 4 & 1 & 5 & 4 & 2 & 4 & 5 \\
\hline
\end{tabular}

Source: the national registers for causes of death 
Figure 4.1.9 Autopsy rates as a percentage of all deaths, 2000-2014

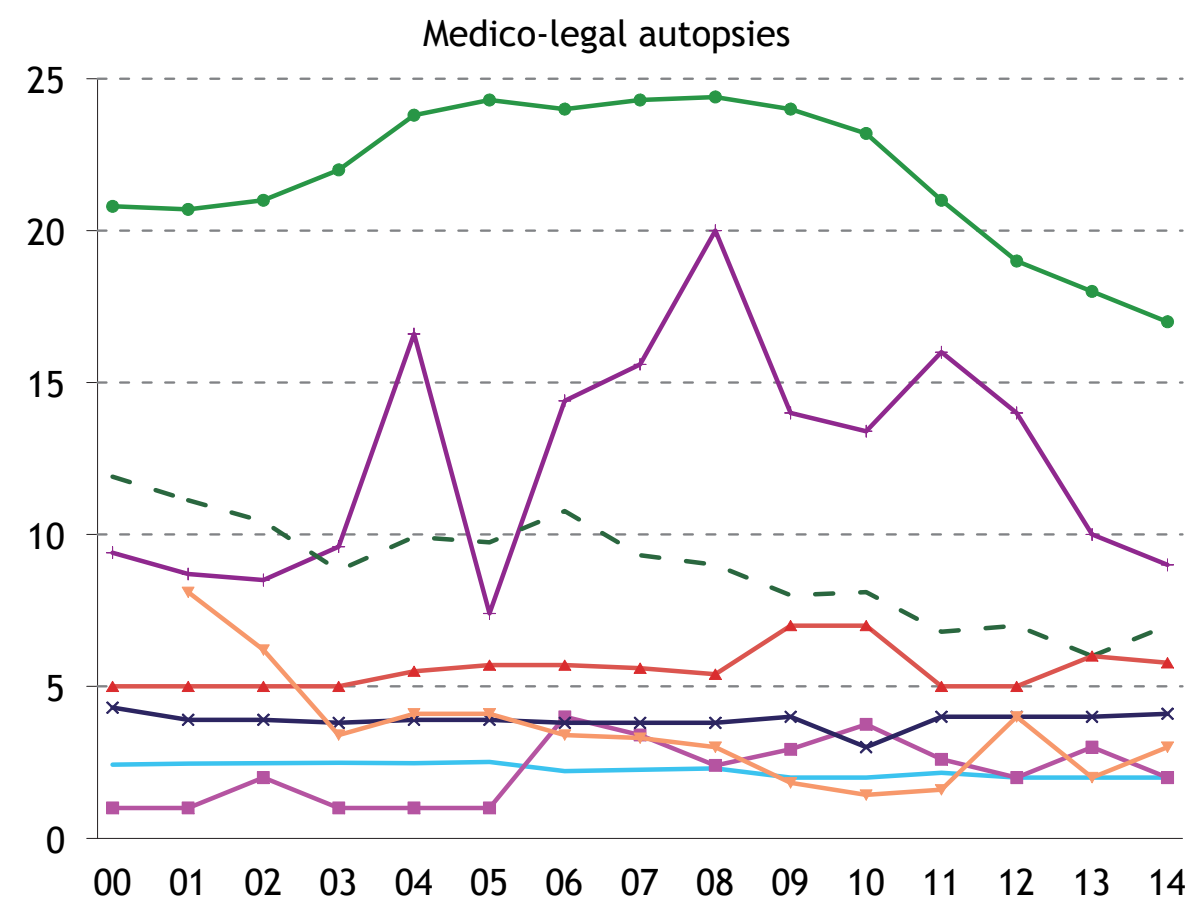

Other autopsies

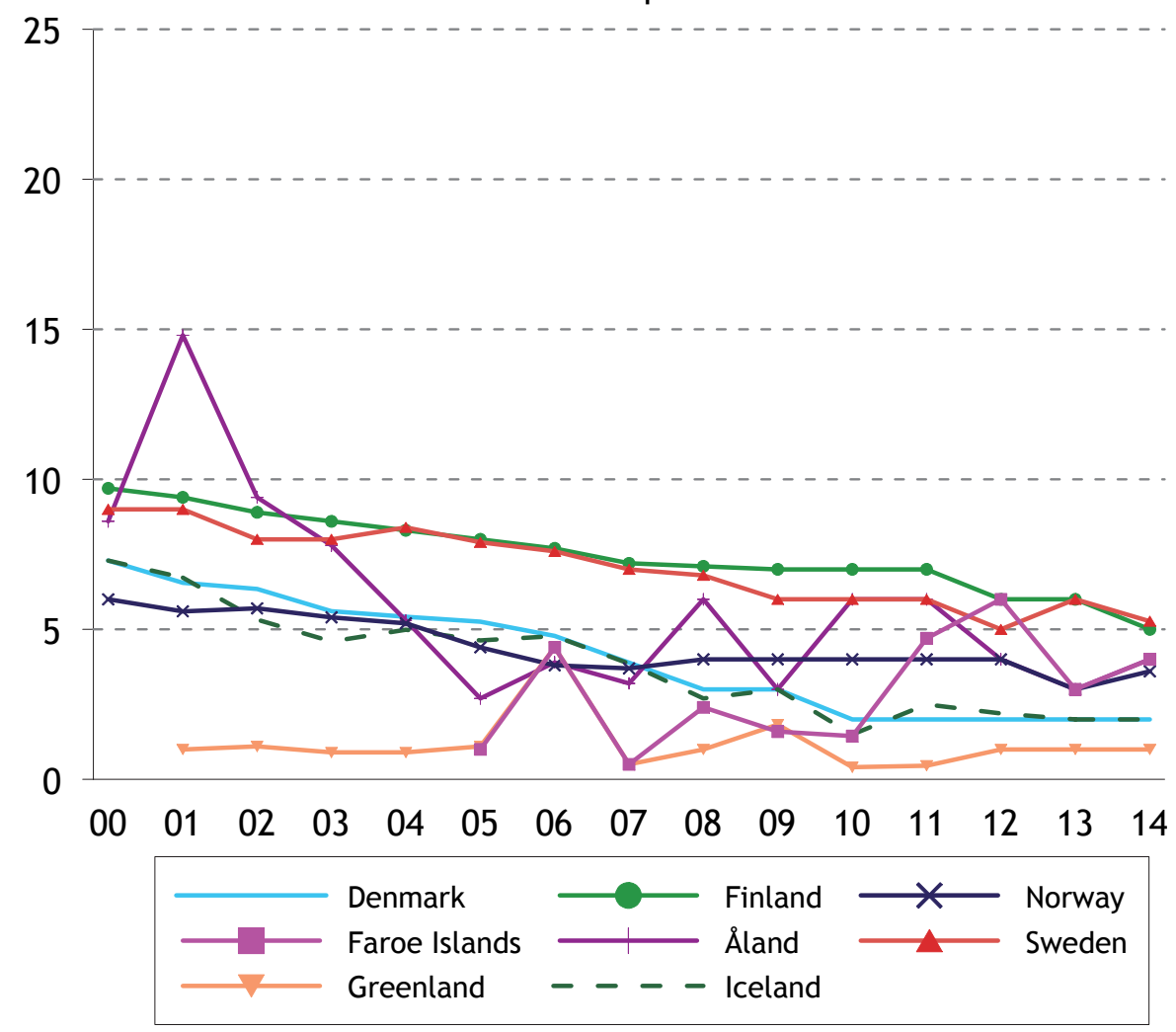

Source: the national registers for causes of death 
Mortality and Causes of Death 


\title{
Chapter 5
}

\section{Resources}

\author{
Extra material \\ OECD: www.oecd.org/els/health-systems/health-data.htm
}

\section{Introduction}

This chapter describes available resources and utilization of resources in the health sector. It begins with a description of the financing of health services, including user charges. This is followed by an overview of total health care expenditure and a description of health care personnel, and capacity and services in hospitals.

\subsection{Financing of health services}

In the Nordic countries, health services are mainly financed by the public authorities. In Iceland and Greenland, financing is primarily provided by the government, while financing in the other countries mainly comes from county and/or municipal taxes and block grants from the governments. With the exception of Greenland, citizens in the Nordic countries contribute directly to the financing, partly through insurance schemes, partly by paying user charges. Only Denmark and Norway use DRG (diagnosis-related groups) in their financing models.

\section{DENMARK}

In the case of Denmark, the Structure Reform resulted in the regions becoming responsible for the health sector from 1 January 2007. A new financial system for the regions was consequently agreed upon. About three quarters of the regions' expenditure is financed through block grants from the state. The rest is financed through a basic contribution from the municipalities, along with municipal and state subsidies that are dependent on activity.

\section{THE FAROE ISLANDS}

The health care system is mainly based on publically provided and financed services, as the private part of health services is limited to e.g. dental care and physiotherapy.

A fixed duty and an income related duty is imposed on citizens.

The municipalities are responsible for providing and financing practitioners' facilities.

\section{FINLAND}

The health care system is highly decentralized. Responsibility for providing health care is devolved to the municipalities (local government). The publicly funded system is divided into three levels: municipal health care, private health care and occupational health care. Alongside this is a much smaller private health care system. 
Municipal financing is based on taxes while the National Health Insurance financing is based on compulsory insurance fees. Municipalities fund primary health care services and the National Health Insurance funds for example private health care, occupational health care, out-patient pharmaceutical products and transport costs. Also most health-related benefits, such as sickness benefits and maternity benefits, are funded through the National Health Insurance Scheme.

\section{ÅLAND}

Åland's health care unit (ÅHS) under Åland's county is responsible for public health care in Åland.

\section{ICELAND}

The Icelandic health care system is mainly financed by the Central Government and by social security funds. Primary care and hospital care are mainly provided by public institutions financed through the state budget and relatively low user charges. Services provided by privately practicing medical specialists are based on fee-for-service schemes financed by the Icelandic Health Insurance and user charges.

\section{NORWAY}

A financing model for somatic hospitals was established in Norway (as from 1 July 1997) that combines block grants and fee-for-service financing. The scheme is regularly evaluated and adjusted. Fee-for-service financing is based on the principle that a service provider (i.e. the hospital) is paid on the basis of services rendered. The scheme involves the state reimbursing a percentage of the average DRG expenses (Diagnosis Related Groups) in connection with treatment of patients.

\section{SWEDEN}

The state is responsible for overall health policy and provides block grants to the county authorities for provision of health services. The largest proportion of funding for health services comes from taxes. Most of the funding for services provided by the county authorities comes from county taxes, and the rest from block grants from the state. Each county authority decides the level of county taxes itself, and how funding shall be allocated. The county authorities also receive revenue from user charges and sale of services. The largest proportion of the budget of the county authorities is used to provide health services and dental services. 
Figure 5.1.1 Distribution of health expenses after funding, percentage of total expenses, 2013

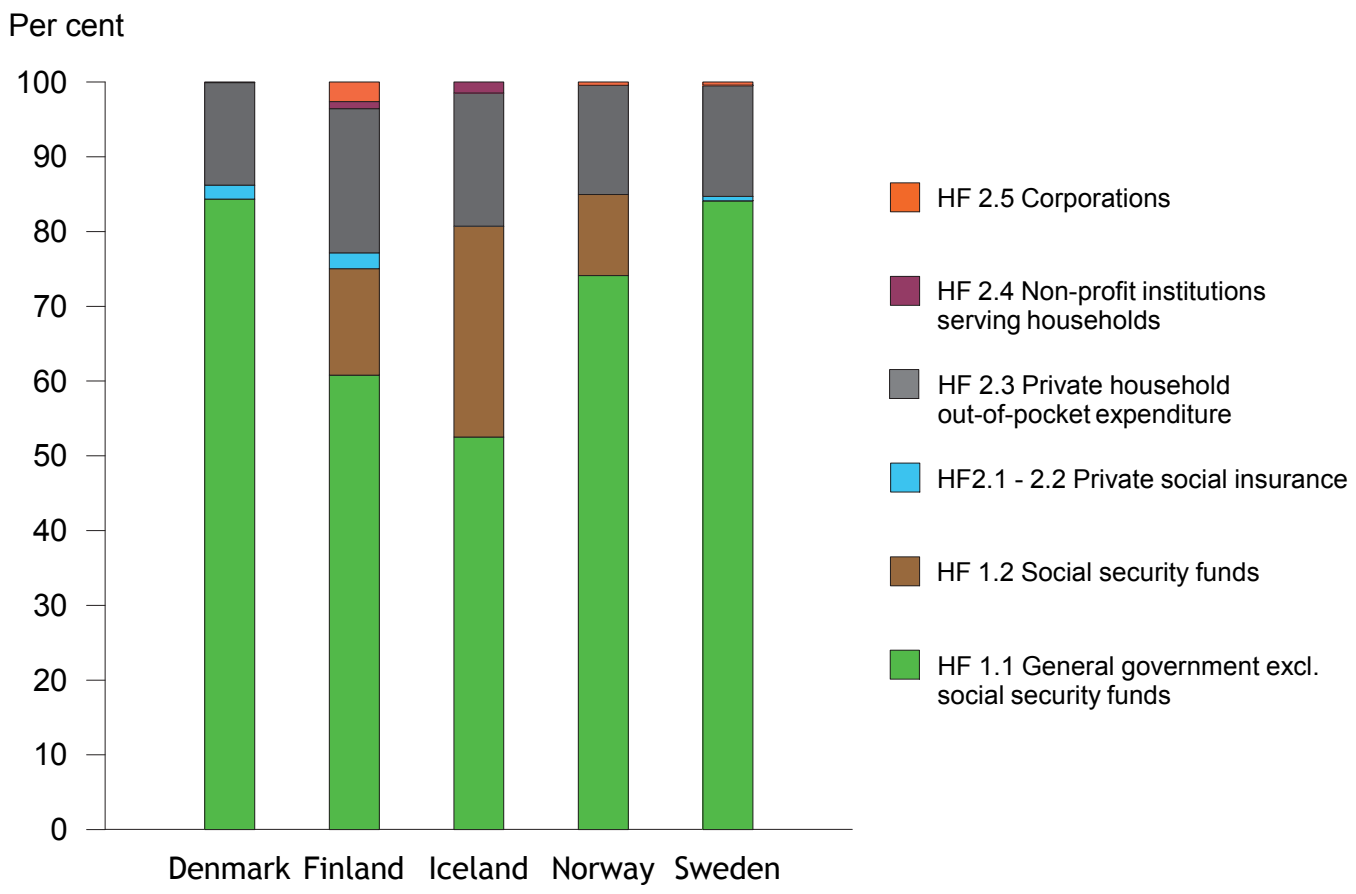

Source: OECD Health Statistics

\subsection{Charges for health care services per 1 January 2016}

\section{Medical visits}

DENMARK, FAROE ISLANDS and GREENLAND

No user charges are payable in Denmark, the Faroe Islands and Greenland.

\section{FINLAND}

The following charges are payable for primary care at health centres:

- A fixed annual user charge of no more than EUR 41.70 in a year, or

- A fixed user charge per visit of no more than EUR 20.90. The user charge is payable for the first three visits to the same health centre in the same calendar year only

A user charge of EUR 28.70 is payable for visits to health centres on working days between $8 \mathrm{pm}$ and 8 am and for visits on Saturdays, Sundays and public holidays. The charges do not apply to people under the age of 18 .

Reimbursement of private physicians' fees is based on fixed charges. The National Social Insurance Institution reimburses a fixed amount of the physician's fee, an amount which is considerably lower than the actual charge. 


\section{ÅLAND}

For medical consultations within the primary health service at a clinic, at specialized health centres and for home visits, there is a user charge of EUR 27. The user charge for a visit to a casualty department is EUR 40. Children and young people under the age of 18 pay half of the user charge. If there is a waiting period of 45 minutes or more in connection with a scheduled visit, the user charge is reimbursed.

\section{ICELAND}

Preventive health care consultations for pregnant women and parents with infants are free of charge and so is school health care. The user charge for a consultation in a health centre and with a private general medical practitioner during normal working hours is ISK 1200 , ISK 960 for 67-69-year-olds who do not have a pension or who have a reduced pension and ISK 600 for other pensioners, disabled people and longterm unemployed people. There is no user charge for children under 18 . Outside normal working hours, the charges are ISK 3 100, ISK 2400 and ISK 1 500. Charges for home visits are ISK 3 400, ISK 2600 and ISK 1600 during day time, while user charges for evenings and nights are ISK 4 500, ISK 3800 and ISK 2200.

The user charge for a consultation with a specialist is ISK 5700 plus 40 per cent of the remaining cost of the consultation, ISK 4400 plus 13.3 per cent of the remaining cost of the consultation for 67-69-year-olds who do not have a pension or who have a reduced pension, ISK 2100 plus 13.3 per cent of the remaining cost of the consultation for other pensioners, disabled people and long-term unemployed people. The user charge for children under 18 years is one ninth of the total charge with a minimum of ISK 890 . There is no user charge for disabled and chronically ill children.

The maximum charge is ISK 35200 in all cases. The same user charges apply for out-patient treatment in hospitals (with the exception of children, for whom there is no user charge). Different user charges apply for treatment in emergency units and with other physicians, and for laboratory tests, radiographs and diagnostic examinations. User charges for persons who have been continuously unemployed for a period of 6 months or longer are the same as for pensioners.

\section{NORWAY}

As a member of the National Insurance scheme, patients only pay a fixed part of the cost of public health services. This applies to medical treatment, buying medicines on a refundable prescription (a so-called blue prescription), physiotherapy, seeing a psychologist and travel expenses to consultations and treatment appointments. If patient have paid a certain amount in user charges, they are eligible for an exemption card. This means that they are exempt from paying user charges for the rest of the calendar year. There are two types of exemption card. The amounts are set annually by the Norwegian Parliament, the Storting. The exemption card for user charge group 1 covers approved user charges paid to doctors, psychologists and outpatient clinics, and for $\mathrm{x}$-rays, patient travel and blue prescription medicine and equipment. The exemption card for user charge group 2 covers approved user charges for physiotherapy, certain dental diseases, treatment in approved rehabilitation institutions and travel for treatment abroad organized by Oslo University Hospital HF - Rikshospitalet. 
Patients are exempt from user charges in connection with check-ups during pregnancy, examinations and treatment for children under the age of 16, psychotherapy for children and young people under the age of 18 and treatment of infectious diseases that are a danger to public health or suspicion of such diseases.

User charges apply for consultations with general practitioners and specialists, out-patient treatment at hospitals, and treatment from a doctor on call. User charges for consultation with general practitioners/doctors on call are: general practitioner: NOK 141 (day) and NOK 238 (night). User charges are NOK 187 (day) and NOK 282 (night) if the doctor is a specialist in general practice. For other medical specialists the user charge is NOK 320 . User charges for home visits are: general practitioners NOK 190 (day) and NOK 304 (night), specialist in general practice: NOK 222 (day) and NOK 336 (night). The user charge for laboratory tests, histological tests and cytology tests is NOK 50. The user charge for x-rays and ultrasound examinations is NOK 227.

\section{SWEDEN}

In Sweden, county authorities can decide the level of user charges for different types of visit and treatment. In 1981, the cost ceiling system was introduced in the health care services. The cost ceiling is regulated in the Act on health care services and applies to all counties. The present system was introduced in 1997, with separate cost ceilings for out-patient visits to the doctor (SEK 900) and for pharmaceutical products (SEK 1800 ) with a successive reduction of user charges for pharmaceutical products. If a parent has several children under 18 years of age, the children are exempt from charges when the total expenses reach SEK 900.

User charges for primary health care vary from SEK 100 to SEK 200 per visit. An extra charge of between SEK 0-150 is payable for home visits, and of SEK 0-100 for telephone prescriptions.

User charges for out-patient consultations with a specialist vary from SEK 230 to SEK 320. If the patient has a referral from the primary health service, the user charge is between SEK 80 and SEK 300 per visit.

User charges for visits to an emergency unit vary from SEK 200 to SEK 300. Nearly all the county authorities have decided that children and young people under the age of 20 are exempt from paying user charges for out-patient treatment. This exemption lasts until the young person's 20th birthday. 
Table 5.2.1 User charges for a consultation with a physician

\begin{tabular}{|c|c|c|c|c|}
\hline & $\begin{array}{l}\text { Are there } \\
\text { consistent } \\
\text { rules for the } \\
\text { whole coun- } \\
\text { try? }\end{array}$ & Amount of user charge & Deviations & $\begin{array}{l}\text { User charge as a } \\
\text { percentage of the } \\
\text { total cost of medi- } \\
\text { cal visits }\end{array}$ \\
\hline Denmark & Yes & - & No & - \\
\hline $\begin{array}{l}\text { Faroe } \\
\text { Islands }\end{array}$ & Yes & - & No & - \\
\hline Greenland & Yes & - & No & - \\
\hline Finland & Yes & $\begin{array}{l}\text { Public: EUR } 0-20.90 \text {. } \\
\text { EUR } 28.70 \text { for visits } \\
\text { between } 8 \text { pm and } 8 \text { am } \\
\text { on weekdays or on Sat- } \\
\text { urdays, Sundays or pub- } \\
\text { lic holidays. Private: } \\
\text { min. } 60 \text { per cent }\end{array}$ & $\begin{array}{l}\text { No charge for children } \\
\text { under the age of } 18 \\
\text { years }\end{array}$ & 13 per cent \\
\hline Åland & Yes & $\begin{array}{l}\text { EUR } 27 . \text { Children and } \\
\text { young people under the } \\
\text { age of } 18 \text { years pay half } \\
\text { the price }\end{array}$ & $\begin{array}{l}\text { Free treatment after } \\
\text { paying EUR } 375 \text {. Free } \\
\text { treatment for children } \\
\text { under } 18 \text { and people } 65+ \\
\text { and disability pensioners } \\
\text { and persons receiving } \\
\text { full-time rehabilitation } \\
\text { benefits after paying } \\
\text { EUR } 120\end{array}$ &.. \\
\hline Iceland & Yes & $\begin{array}{l}\text { ISK } 1200-4500 \text { in pri- } \\
\text { mary care, other fees } \\
\text { for specialized care }\end{array}$ & $\begin{array}{l}\text { Half the amount of ISK } \\
600-2200 \text { for pension- } \\
\text { ers, disabled and long- } \\
\text { term unemployed peo- } \\
\text { ple. ISK } 960-3800 \text { for } \\
67-69 \text {-year-olds with no } \\
\text { or a reduced pension. } \\
\text { No charge for children } \\
\text { under the age of } 18 \\
\text { years }\end{array}$ & \\
\hline Norway & Yes & $\begin{array}{l}\text { Consultation with: a } \\
\text { general practitioner: } \\
\text { NOK } 141 \text { (day), } \\
\text { NOK } 238 \text { (even- } \\
\text { ing/night), with a spe- } \\
\text { cialist: NOK } 320\end{array}$ & $\begin{array}{l}\text { No charge for children } \\
\text { under } 16 \text { years }\end{array}$ & 35 per cent \\
\hline Sweden & No & $\begin{array}{l}\text { Primary health services, } \\
\text { general practitioners } \\
\text { 100-300 SEK, Specialists } \\
\text { 100-350 SEK }\end{array}$ & $\begin{array}{l}\text { Yes. Nearly all counties } \\
\text { have decided that chil- } \\
\text { dren and young people } \\
\text { un-der the age of } 20 \text { do } \\
\text { not pay user charges for } \\
\text { out-patient treatment }\end{array}$ & .. \\
\hline
\end{tabular}

Source: SV, the Swedish Association of Local Authorities and Regions, SALAR 


\section{Pharmaceutical products}

\section{DENMARK}

There are no fixed percentages for reimbursement of the cost of pharmaceutical products in Denmark, as the reimbursement depends on the amount of pharmaceutical products used by the individual patient. The percentage of reimbursement increases proportionally with the patient's use of pharmaceutical products.

Reimbursable pharmaceutical products are products with a documented and valuable therapeutic effect for a clear indication, where the price of the pharmaceutical product is reasonable in relation to its therapeutic value.

An individually assessed subsidy may be granted for pharmaceutical products that are not subject to a general subsidy by submitting an application through one's own doctor to the Danish Medicines Agency.

The Danish Medicines Agency determines a reference price for each group of pharmaceutical products covered by the reference price system. The reference price forms the basis for the calculation of the subsidy.

The subsidy is calculated on the basis of the reference price of each packet. Thus, the subsidy cannot be higher than the actual cost of the pharmaceutical product. Subsidies based on need are not changed.

The aim of the system is that physicians and dentists choose the cheapest product on the market (substitution). In special cases, the physician or dentist can choose not to substitute, if he or she finds that substitution by the pharmacy is not appropriate.

Current prices are determined for all pharmaceutical products on the market that have a marketing licence.

Since the liberalization in October 2001, there are now more than 1500 authorized agents for non-prescription pharmaceutical products for people and/or animals.

All authorized businesses, irrespective of the selection of pharmaceutical products, that they sell, must follow the current regulations relating to storage and quality of pharmaceutical products, and the prohibition against self-service sale and sale to children under 15 years of age.

In addition, agents for non-prescription pharmaceutical products for people shall offer a basic selection of goods, determined by legislation. For certain nonprescription pharmaceutical products, such as drugs for pain relief, no more than one packet can be sold per customer per day.

A list of pharmaceutical products that can be sold outside pharmacies can be found on the web site of the Danish Medicines Agency: www.laegemiddelstyrelsen.dk

\section{FAROE ISLANDS}

Part of the cost of pharmaceutical products is covered by the public sector, and part is covered by user charges. Only pharmaceutical products that are on the subsidies list are reimbursed. The subsidies list is administered by the Chief Pharmaceutical Officer. Reimbursements on the Faroe Islands are not a fixed percentage of the cost, as they are dependent on the level of consumption of the pharmaceutical product for each patient. The subsidy is adjusted according to the consumer price index. The percentage increases with the patient's consumption of pharmaceutical products. For 
an annual purchase under DKK 516, the full cost of the pharmaceutical product is paid by the consumer, while costs of more than DKK 5469 are fully subsidized by the public sector (maximum user cost after subsidy is DKK 2 090). However, the costs for patients over the age of 67 years are fully subsidized from DKK 2 456, and the maximum cost for the user after subsidy for persons $67+$ is DKK 1340 . Costs for children under 18 years are fully subsidized from the first DKK, and therefore children pay nothing for prescribed medicine eligible for subsidy. In accordance with the Social Security Act, subsidies for purchase of pharmaceutical products are also granted to persons who are not able to bear the costs themselves. Pharmaceutical products prescribed in hospitals are always free of charge.

\section{GREENLAND}

All pharmaceutical products are distributed through the health service except for certain non-prescription pharmaceutical products. These are available, to a very limited degree, from certain general stores. Non-prescription pharmaceutical products are distributed to a varying degree by district health services. Pharmaceutical products distributed by the health services are free.

\section{FINLAND AND ÅLAND}

There are three payment categories (40, 65 and 100 per cent) for prescription pharmaceutical products, and reimbursement is calculated separately for each purchase after an annual initial deductible of EUR 50.00 for all adults. However, there is a user charge of EUR 4.50 for pharmaceutical products with 100 per cent reimbursement.

The reimbursement amount depends on whether or not the pharmaceutical product is part of the reference pricing system. Pharmaceutical products are categorized according to the reference pricing system. Products that belong to the same reference pricing group contain equal amounts of the same drug substance and are biologically equivalent, which makes them interchangeable.

Some new and expensive drugs (e.g. for dementia and multiple sclerosis) are paid for by the hospital or municipality in special cases. New drugs are not automatically covered by the reimbursement scheme, and many drugs are marketed without any reimbursement. Health economists have gained more and more influence as to which products should be reimbursed.

In addition to reimbursement for medicines, reimbursement can also be given for special diets for some treatment-intensive diseases and for ointments used in the treatment of chronic skin diseases.

As a main rule, the health insurance scheme reimburses expenditure on prescription pharmaceutical products exceeding EUR 610.37 in the course of one calendar year (excluding user charges of EUR 2.50 per product per purchase). 


\section{ICELAND}

The subsidy system for pharmaceutical products in Iceland is similar to the one in the other Scandinavian countries (Denmark, Faroe Islands, Norway and Sweden). The system builds on payment contribution steps, where the individual pays proportionally less as the costs for pharmaceutical products increase during a 12-month period. The individual pays all expenses for pharmaceutical products up to a certain limit (the subsidy limit). Then his or her payment gradually decreases until annual expenses have reached a maximum amount (the annual limit). After this the expenses are fully covered.

The 12-month payment period starts with an individual's first purchase of a pharmaceutical product. The patient pays the initial ISK 22 000, then 15 per cent of the costs up to ISK 31750 , then 7.5 per cent of the costs up to ISK 62000 . Costs that exceed this amount are fully subsidized. The annual limits for subsidies to pensioners, disabled people, children and young people under the age of 22 years are lower than for other people. These groups pay the initial ISK 14500 , and their costs are fully subsidized when they have paid ISK 41000.

All pharmaceutical products authorized by the Health Insurance scheme are included in the payment system. Other pharmaceutical products fall outside the payment system.

\section{NORWAY}

There are two types of reimbursement scheme for pharmaceutical products: reimbursement authorized in advance (blue prescription) and partial reimbursement with contribution (white prescription).

Blue prescription: Most pharmaceutical products are reimbursed according to a system based on diagnoses and approved pharmaceutical products prescribed by a physician.

User charges for pharmaceutical products on blue prescription are 38 per cent of the prescription cost, up to a maximum of NOK 520 per prescription up to a quantity corresponding to 3 months' use.

White prescription: Normally the patient pays the full cost of pharmaceutical products on a white prescription. In some cases, the cost can be partially reimbursed through the reimbursement scheme. The patient pays the full cost of the pharmaceutical product at the pharmacy. When the cost has reached a maximum amount, the patient can apply to have further costs reimbursed.

The National Insurance Scheme covers 90 per cent of expenses exceeding the maximum limit.

The maximum limit for ordinary reimbursement is NOK 1667.

\section{SWEDEN}

Certain pharmaceutical products are included in the cost ceiling arrangement. This means that part of the cost of the pharmaceutical product is refunded by the state through taxation. The Dental and Pharmaceutical Benefits Agency (TLV) is a state authority whose remit is to determine which pharmaceutical products, disposable items and dental treatment shall be included in the cost ceiling arrangement. Different types of pharmaceutical products are included in the cost ceiling arrangement, 
including disposable items and contraceptives. Some non-prescription pharmaceutical products are also included in the cost ceiling arrangement.

According to the legislation, pharmacies have a duty to substitute pharmaceutical products with cheaper generic alternatives. Generic alternatives are pharmaceutical products that have been approved by the Medical Products Agency as having the same function, quality and safety as the original pharmaceutical product.

User charges, i.e. the part of the cost paid for by the patient, are as follows:

- the whole cost up to SEK 1100

- 50 per cent of the cost in the range SEK $1100-2100$

- 25 per cent of the cost in the range SEK $2101-3900$

- 10 per cent of the cost in the range SEK 3900 - 5400

- 0 per cent of costs exceeding SEK 4300

When a patient has paid a total of SEK 2200 in a 12-month-period, the patient receives pharmaceutical products and disposable items free of charge for the rest of the period. 
Table 5.2.2 User charges for pharmaceutical products

\begin{tabular}{|c|c|c|c|c|}
\hline & $\begin{array}{l}\text { Are there } \\
\text { consistent } \\
\text { rules for the } \\
\text { whole coun- } \\
\text { try? }\end{array}$ & Amount of user charge & Deviations & $\begin{array}{l}\text { User charge as \% of } \\
\text { the total cost of } \\
\text { the pharmaceuti- } \\
\text { cal product }\end{array}$ \\
\hline Denmark & Yes & $\begin{array}{l}\text { Reimbursement depend- } \\
\text { ent on the level of the } \\
\text { patient's consumption of } \\
\text { drugs in the primary } \\
\text { sector }\end{array}$ & No & .. \\
\hline $\begin{array}{l}\text { Faroe } \\
\text { Islands }\end{array}$ & Yes & $\begin{array}{l}\text { Reimbursement depend- } \\
\text { ent on the level of the } \\
\text { patient's consumption of } \\
\text { drugs in the primary } \\
\text { sector }\end{array}$ & $\begin{array}{l}\text { Reimbursement is higher } \\
\text { for persons over the age } \\
\text { of } 67 \text { years or under the } \\
\text { age of } 18 \text { years }\end{array}$ & .. \\
\hline Greenland & Yes & - & No & - \\
\hline Finland & Yes & $\begin{array}{l}60 \text { per cent of the cost } \\
\text { after the annual costs } \\
\text { exceed EUR } 50\end{array}$ & $\begin{array}{l}\text { For certain diseases EUR } \\
4.50 \text { or } 35 \text { per cent of } \\
\text { the cost are paid (dis- } \\
\text { ease specific) }\end{array}$ & 44 \\
\hline Åland & Yes & As in Finland & As in Finland & - \\
\hline Iceland & Yes & $\begin{array}{l}\text { Reimbursement depend- } \\
\text { ent on the level of the } \\
\text { patient's consumption of } \\
\text { drugs in the primary } \\
\text { sector }\end{array}$ & $\begin{array}{l}\text { Pensioners, children } \\
\text { (under } 18 \text { years), young } \\
\text { people (18-22 years old) } \\
\text { and disabled people pay } \\
\text { two thirds of the costs }\end{array}$ & .. \\
\hline Norway & Yes & $\begin{array}{l}38 \text { per cent of the cost, } \\
\text { maximum NOK } 520 \text { per } \\
\text { prescription }\end{array}$ & $\begin{array}{l}\text { No user charge for chil- } \\
\text { dren under } 16 \text { years }\end{array}$ & .. \\
\hline Sweden & Yes & $\begin{array}{l}\text { SEK 0-2200. The high } \\
\text { cost threshold system } \\
\text { decreases patients' } \\
\text { costs for prescription } \\
\text { medicines in stages }\end{array}$ & $\begin{array}{l}\text { From January } 1 \text { st } 2016 \text {, } \\
\text { prescribed drugs in the } \\
\text { reimbursement system } \\
\text { will be free for children } \\
\text { under } 18 \text { years }\end{array}$ & .. \\
\hline
\end{tabular}




\section{Treatment in hospitals}

As shown in the overview, there are no user charges for hospitalization in Denmark, the Faroe Islands, Greenland, Iceland and Norway. In Iceland and Norway, however, there is a charge for specialist out-patient treatment in hospitals, cf. the section on consultations with a physician.

\section{FINLAND}

Patients pay a charge for admission to hospitals and health centres: EUR 41.70 for an out-patient visit and EUR 49.50 for overnight care in somatic department and EUR 22.80 in psychiatric departments. The charge for rehabilitation is EUR 17.10 per treatment day, and the maximum user charge for day surgery is EUR 136.80 plus EUR 49.50, if the patient has to stay overnight. A series of treatment costs EUR 11.50 per visit (max. 45 times per year).

\section{ÅLAND}

The user charge per day for patients who are hospitalized is EUR 33. When the maximum limit has been reached, the user charge is reduced to EUR 15. The maximum limit is EUR 375 for persons between 18 and 64 years, and EUR 120 for persons aged 65 and older and for people with a disability pension.

The user charge per day for persons under the age of 18 is EUR 18. When the maximum limit (EUR 120) has been reached, health care in hospitals is free of charge.

The user charge for day surgery is EUR 66. For medical rehabilitation the user charge per day is EUR 20. When the maximum limit has been reached, health care is free of charge.

The user charge for long-term care in a hospital is calculated on the basis of the patient's ability to pay.

\section{NORWAY}

In-patient hospital treatment is free to all who qualify, but there are user charges for visits to doctors and specialists and for prescription medicines. Citizens must also pay for radiology and laboratory tests and for non-emergency transportation. There are a number of exemptions, for example for people who suffer from chronic diseases, pregnant women and women who have just given birth.

\section{SWEDEN}

The county authorities and the municipalities can largely decide themselves about user charges for a visit to the doctor and for other health services. For a hospital stay, there is a user charge per day of a maximum of SEK 80 . The amount varies in different counties from SEK 0 to 80, depending on the patient's income, age and length of stay.

Most county authorities have no user charges for in-patient treatment in hospitals for persons under 20 years of age.

There are private hospitals in most of the Nordic countries, which provide all or some of their services to the public health service, but according to somewhat different regulations in the different counties. 
Table 5.2.3 User charges for hospitalization

\begin{tabular}{|c|c|c|c|c|}
\hline & $\begin{array}{l}\text { Are there consistent } \\
\text { rules for the whole } \\
\text { country? }\end{array}$ & $\begin{array}{l}\text { Amount of user } \\
\text { charge }\end{array}$ & Deviations & $\begin{array}{l}\text { User charges as a } \\
\text { percentage of the } \\
\text { total cost of } \\
\text { hospitalization }\end{array}$ \\
\hline Denmark & Yes & - & No & $4.2^{1}$ \\
\hline $\begin{array}{l}\text { Faroe } \\
\text { Islands }\end{array}$ & Yes & - & No & - \\
\hline Greenland & Yes & - & No & - \\
\hline Finland & Yes & $\begin{array}{l}\text { Max. EUR } 41.70 \text { per } \\
\text { day for overnight } \\
\text { care and } \\
\text { EUR } 136.80 \text { for day } \\
\text { surgery }\end{array}$ & $\begin{array}{l}\text { For children } 0-17 \\
\text { years max. for } 7 \\
\text { days. Payment for } \\
\text { long-term stay } \\
\text { according to means }\end{array}$ & 7 \\
\hline Åland & Yes & $\begin{array}{l}\text { EUR 33; EUR } 18 \text { for } \\
\text { people under the } \\
\text { age of } 18 \text { years } \\
\text { EUR } 66 \text { for day } \\
\text { surgery }\end{array}$ & $\begin{array}{l}\text { Payment for long- } \\
\text { term stay according } \\
\text { to means }\end{array}$ & .. \\
\hline Iceland & Yes & - & No & $0.6^{1}$ \\
\hline Norway & Yes & - & No & $2.3^{1}$ \\
\hline Sweden & No & SEK 0-100/day & $\begin{array}{l}\text { County councils and } \\
\text { regions decide } \\
\text { charges }\end{array}$ & $1.9^{1}$ \\
\hline
\end{tabular}

1 2013, source: OECD HEALTH STATISTICS

\section{Dental treatment}

In all countries, part of the cost of dental treatment is refunded in the following cases: dental treatment that is necessary to prevent serious complications due to infection in the teeth and periodontium; for immuno-compromised patients, such as patients with leukaemia or head and neck cancer; patients waiting for a transplant, patients who need bone marrow transplants; and patient groups with similar problems.

\section{DENMARK}

Reimbursement is provided by the public health insurance scheme. Adults typically pay 60 per cent of the agreed fees. No subsidy is granted for dentures.

Municipal and regional dental services are regulated by the health legislation.

In addition, approximately 1.9 million Danes are covered by a private insurance scheme. Some schemes provide subsidies for dental treatment.

Children and young people under 18 years of age receive free municipal dental care including orthodontic treatment. Children under 16 years of age who wish to have treatment that is not provided free of charge by the municipal council, may - by paying a user charge - choose to be treated in a private clinic of their own choice or at a public dental clinic in another municipality. From 1 January 2014, elderly people who live in a nursing home or in their own home with technical aids are offered den- 
tal care for which there is a maximum annual charge of DKK 475. In addition, the municipalities provide a subsidy for dentures in cases of impaired function or disfigurement resulting from damage caused by accidents.

The municipality offers specialist dental treatment to persons who because of psychiatric illness or mental health disorders cannot use the existing dental services for children and young people, for adults, or for people needing special care. For these services, the region, from 1 January 2014, charges the patient a maximum of DKK 1 775 per year.

The region offers specialized dental care (regional dental service) or highly specialized dental care (in dental research centres) to children and young people with dental conditions that would lead to a permanent reduction in function if left untreated.

In addition, the region grants a special reimbursement for dental care for cancer patients, who either due to radiation of the head and neck or due to chemotherapy suffer from considerable documented dental problems, and to persons who due to Sjögren's syndrome suffer from considerable documented dental problems. From 1 January 2014, the region can demand a user payment of a maximum of DKK 1775 annually for these services. Finally, the region provides highly specialized dental advice, examination and treatment (in dental research centres) for patients with rare diseases and disabilities, for whom the underlying disease can lead to special problems with their teeth, mouth or jaws.

Oral and maxillofacial surgery is carried out in hospitals and is paid for by the regions in accordance with the health legislation.

In addition to the general rules outlined above, the municipalities can provide support for necessary dental treatment in accordance with the legislation relating to social services.

\section{FAROE ISLANDS}

Dental treatment is mainly provided by private dentists. Payment is therefore partly private, and partly subsidized (about half of the costs) by the public services. The specific amount of the subsidy is regulated by the agreement between the home rule government and the Faroese Dental Association. There is no maximum user charge for dental treatment, as there is for subsidized pharmaceutical products.

The municipalities provide a free dental service for children up to the age of 18 . Until 2014, this service applied only to children up to the age of 16, but the age limit was raised in 2014. This service also provides special dental care, such as orthodontic treatment.

Reimbursement of expenses for treatment of congenital diseases or diseaserelated dental conditions can be claimed according to the social legislation.

\section{GREENLAND}

All public dental care is free of charge. There is limited access to private dentists. All private dental treatment is paid for by the patient. 


\section{FINLAND}

There is a basic user charge of EUR 10.30 per visit for dental treatment at a health centre, EUR 13.30 per visit to a dentist, and EUR 19.40 for a visit to a specialist. In addition to this, user charges of EUR 8.50-225.50 can be charged, dependent on the type of treatment provided.

The health insurance scheme reimburses 60 per cent of the treatment costs within the rates fixed by the Social Insurance Institution for one annual dental examination in the private dental service. Orthodontic treatment is only reimbursed if the treatment is necessary to prevent other illnesses. Expenditure on dentures and dental laboratory costs are not included in the reimbursement scheme.

Expenses for laboratory and X-ray examinations ordered by a dentist are refundable. Expenses for drugs prescribed by a dentist and travelling costs to visit a dentist are refundable under the same terms as for medical prescriptions and travelling costs to visit a physician.

\section{ÅLAND}

All public dental treatment for persons under 19 years of age is free of charge. For others, the user charge for a dental visit is EUR 12 with additional standard fees for treatment and examinations. The patient pays the actual cost of orthodontic treatment and prosthetic treatment. The same rules as in Finland apply for treatment with private dentists.

\section{ICELAND}

The health insurance scheme in Iceland pays according to a rate fixed by the health insurance scheme. This rate is generally different from the rate used by private dentists, as private dentists in Iceland are allowed to set their own fees.

In April 2013, a new contract for dental treatment for children under the age of 18 was signed. According to the agreement parents register their child with a family dentist, who is then responsible for providing the necessary dental care of the child and is fully paid by the national health insurance except for a low annual appointment charge of ISK 2500 that the parents pay. The agreement is being implemented in seven stages until all children from $0-18$ years-old will be covered on $1^{\text {st }}$ of January 2018. Until then other children receive a 75 per cent subsidy for their dental treatment (according to health insurance rates), with the exception of gold and porcelain crowns, dental bridges and orthodontic treatment. A special grant will be given to children in need who are not yet covered by the agreement if they cannot afford the necessary dental treatment. Orthodontic treatment is not covered by the agreement. Subsidies for orthodontic treatment can reach ISK 150000 according to special rules.

The health insurance scheme offers partial reimbursement of the cost of dental treatment for persons aged 67 years or older.

People suffering from chronic illnesses, pensioners and disability pensioners are also eligible to receive a partial subsidy for their costs.

For this group, subsidies of 50, 75 or 100 per cent are provided for the cost of dental treatment (according to health insurance rates). Full dentures and partial dentures are covered. Gold and porcelain crowns, dental bridges and implants can be reimbursed by up to ISK 80000 annually. 
Implants are also included for those who cannot use a full denture. A partial subsidy is provided for pensioners who cannot use a full denture due to alveolar bone resorption or other problems.

95 per cent of the cost of treatment (incl. orthodontics) of serious congenital disfiguration and serious anomalies such as cleft palate and aplasia, and of the damage caused by accidents and illnesses, are reimbursed according to special rules.

No subsidy is provided for dental treatment to the rest of the population. Furthermore, no private dental insurance is available.

\section{NORWAY}

Most people pay the cost of dental treatment themselves.

Adults over 20 years of age normally pay for their own dental treatment.

When dental treatment is needed because of several defined diseases/conditions /injuries, the patient can receive reimbursement/benefit from the National Insurance Scheme. The public dental service offers free treatment to the following groups:

- children and young people under the age of 18 years

- people with mental disabilities

- elderly people, people with chronic illnesses and disabled people who are either living in institutions or receiving home nursing services

- other groups of people with special needs, e.g. people in prison

Adolescents 19-20 years of age receive subsidized dental care. The county authorities cover a minimum of 75 per cent of the cost of dental treatment for this group.

The National Insurance Scheme covers part of the cost of necessary orthodontic treatment for children up to the age of 18.

\section{SWEDEN}

According to the Act relating to dental services, children and young people have the right to regular and comprehensive dental care until and including the calendar year in which they reach 19 years of age. Comprehensive dental care means that young people under 20 years of age shall receive general dental care and specialist dental care.

The current dental subsidy system was introduced on 1 July 2008 and expanded with support for certain patient groups from 1 January 2013.

The system consists of:

- A general dental subsidy

- A special dental subsidy

- A cost ceiling

The aim of the general subsidy is to encourage adults to regularly visit a dentist for examination and preventive care. The annual subsidy depends on age:

- For 20-29 year-olds the subsidy is 300 SEK

- For 30-74 year-olds the subsidy is 150 SEK

- For people 75 years and older the subsidy is 300 SEK 
All adults are also included in the cost ceiling arrangement. The cost ceiling means that patients have to pay only a part of the cost for expensive treatment. The Dental and Pharmaceutical Benefits Agency regulates which care is covered. For every treatment measure covered by the cost ceiling, a reference price is specified from which reimbursement is calculated. For costs above 3000 SEK the patient is reimbursed the following:

- 50 per cent of costs exceeding 3000 SEK, calculated from the reference price

- 85 per cent of costs exceeding 15000 SEK, calculated from the reference price

Adults with specific illnesses, elderly people and people with functional disabilities, have the right to receive reimbursements for dental treatment from the county authorities. This includes reimbursement for preventive care, necessary treatment, dental treatment that is part of the treatment of a disease, and dental aids.

Apart from providing free dental treatment for children and young people, the county authorities and the regions have responsibility for: oral surgery in hospitals, dental treatment that is part of the treatment of a disease, and dental treatment for people who have difficulty in maintaining their own oral health. Special regulations for reimbursement of dental expenses apply for these groups.

\section{Maximum user charges}

\section{DENMARK}

There are no rules in Denmark for maximum user charges, with the exception of pharmaceutical products and dental treatment (cf. the section on reimbursement for dental treatment).

\section{FAROE ISLANDS}

For subsidized medicine, there is a maximum user charge of DKK 2090 annually (no charges for children under 18 years and DKK 1340 for pensioners). There is no maximum user charge for dental treatment. Apart from pharmaceutical products and dental care, there are no user charges in the Faroe Islands (cf. the sections on reimbursement for pharmaceutical products and reimbursement for dental treatment).

\section{GREENLAND}

There are no user charges in Greenland with the exception of non-prescription medicines and some types of dental treatment (cf. the sections on reimbursement for pharmaceutical products and reimbursement for dental treatment). There are no maximum user charges.

\section{FINLAND}

If the total cost of pharmaceutical products exceeds EUR 610.37 per year, or if travelling costs for treatment exceed EUR 300 per year, the Social Insurance Institution reimburses the excess costs.

If a person's ability to pay taxes is reduced because of sickness, a special tax relief may be granted. The amount of the tax relief is calculated on the basis of the person's and his/her family's ability to pay taxes. 
User charges for a long-term stay in an institution or a hospital cannot exceed 85 per cent of a patient's/resident's net income per month. If the spouse with the highest income is hospitalized, the user charge for the hospitalization cannot exceed 42.5 per cent of the spouse's joint net income per month. A patient must have at least EUR 107 per month for personal necessities. The same user charge is payable in all kinds of institutions within the social and health care sectors.

A user charge ceiling of EUR 691 is applied by the municipal social and welfare sectors. Once the ceiling for the present calendar year is exceeded, the user may generally utilize services free of charge. The ceiling applies to physician services in the primary health care sector, physiotherapy, out-patient treatment, day surgery and short-term stays in institutions in the social and health sectors. Dental care, patient transport, certificates, laboratory tests and radiological examinations requisitioned by private physicians must still be paid for. Income-regulated payments are not included in the maximum amount.

User charges for children under 18 years of age are added to the amount paid by the person who has paid the user charges.

\section{ÅLAND}

The rules for maximum user charges for pharmaceutical products and transport to and from treatment are the same as in Finland.

The maximum user charge for health care and out-patient treatment is EUR 375 within one calendar year, after which there is no charge for the remainder of the year, with the exception of short-term stays in institutions/hospitals, where the charge is reduced from EUR 33 per day to EUR 15 per day.

For children and young people under the age of 18 and people over the age of 65, the maximum amount for user charges is EUR 120 per calendar year. After this amount has been reached, all treatment for children and young people is free. The user charge per day for a hospital stay for persons aged 65 years and older is reduced from EUR 33 to EUR 15.

As part of the maximum user charge, payment for out-patient treatment and services received outside the county are also included. Dental treatment and X-ray and laboratory examinations are not included. User charges may be deducted from municipal tax.

\section{ICELAND}

User charges for people aged 18-70 years and for unemployed people are reimbursed if the costs exceed ISK 35200 during one calendar year.

The same applies to children under 18 if user charges exceed ISK 10700.

User charges exceeding ISK 28200 are reimbursed for people aged 67-69 who have either no pension or reduced pension.

User charges exceeding ISK 8900 are reimbursed for the following groups: people aged 60-70 who receive a full basic pension, pensioners aged 70 years or older, and disabled people.

If there are one or more children under the age of 18 in one family, they count as one person in relation to the user charge ceiling. 
When the user charge ceiling has been reached, an insured person receives a discount card, which guarantees full or partial reimbursement for the rest of the year, according to certain rules.

The user charge ceiling scheme covers the following services: consultation with a general medical practitioner or a specialist, home visit by a physician, out-patient treatment in a hospital or a casualty department, and laboratory examinations and Xray treatment. The scheme does not cover treatment for in vitro fertilization.

NORWAY: When a patient has paid user charges up to a certain amount, he or she receives an exemption card. All further treatment is then free for the rest of the year.

There are two exemption schemes in Norway, exemption scheme 1 and exemption scheme 2 . They cover different health services.

User charges for the following are included in exemption scheme 1:

- treatment from a medical practitioner

- treatment from a psychologist

- out-patient treatment

- x-ray examination

- travel costs

- pharmaceutical products (blue prescription)

User charges for the following are included in exemption scheme 2:

- examination and treatment by a physiotherapist

- certain types of dental treatment

- stays in approved rehabilitation institutions

- travel abroad for treatment under the auspices of Oslo University Hospital HF Rikshospitalet

The cost ceiling is NOK 2185 for exemption scheme 1 and NOK 2670 for exemption scheme 2 in 2015.

SWEDEN

Special regulations apply for the cost ceiling arrangement for pharmaceutical products and health care. 


\subsection{Health care expenditure}

\section{Development of health care expenditure}

Health plays a central role in peoples' everyday lives and is an issue that people are concerned about. Thus, health is often a topic for debate, and health issues receive much attention in the press. Attention is particularly focussed on production of health services. Questions are asked about whether health services are adequate and about what health care costs society and individuals. The increasing cost of health care is an issue of concern in many countries. According to the OECD, the reason for this concern is that health services are mainly publicly financed and so increasing health care expenditure is an extra burden on public budgets. If priorities are not changed, this will lead to higher taxes for both citizens and companies.

In the Nordic countries, between 75 and 85 per cent of health care expenditure is publicly financed. In 2013, the level of public financing was lowest in Finland.

Measured in relation to gross domestic product (GDP), health care expenditure has been relatively stable or has shown a slight increase in the second half of the 1990s and the beginning of this century. Health care expenditure represents between 8 and 9 per cent of GDP.

Table 5.3.3 shows health care expenditure per inhabitant, which was highest in Norway and lowest in Greenland.

\section{Changes in the recording of health care expenditure}

Health care expenditure includes all expenditure, both private and public, on consumption or investment in health services, etc. The expenditure can be financed by both private and public sources, including by households. Examples of health care expenditure by households are the cost of spectacles, orthopaedic items, pharmaceutical products, dental treatment, medical treatment, physiotherapy services and other health services. Other types of expenditure include national insurance or private insurance reimbursements for use of health services, and public expenditure (net) on hospitals and primary health services.

Public expenditure on preventive measures and administration of health services is included. Expenditure on running private hospitals that are not included in the public budget is also included.

Health care expenditure also includes part of the expenditure on nursing and care for elderly people and people with disabilities. According to international guidelines, this applies to the part of expenditure on nursing and care that can be specified as expenditure related to health. Services for elderly people and people with disabilities are often integrated, and it can be difficult to draw clear boundaries between what should be defined as expenditure on health services and what should be defined as expenditure on social services. What is included as expenditure on health services can vary for the different countries.

There will always be such problems when one compares statistics from several countries. This does not mean that comparisons are worthless, but one must be 
aware that some of the observed differences can be the result of different definitions and boundaries.

In order to ensure the best possible comparability of statistics, international organizations such as the OECD, the UN and EUROSTAT work on producing classifications, standards and definitions. For example, the OECD have developed "A System of Health Accounts". This accounting system has been developed in order to meet the political needs for data, and also the needs of researchers in this area. The common framework that the system is built on will ensure that the comparability of data between countries and over time is as good as possible. The system has also been developed to provide comparable statistics, independently of how health services are organized in the countries.

All the Nordic countries have implemented, or are in the process of implementing, OECD's system of health accounts, and the figures presented in this publication are based on this system. Not all the countries have come equally far in implementing the system, and it is not certain at the moment how comparable the various national health accounts are. Therefore, the unsolved problems faced by the countries, and the different solutions they have found, must be taken into account when interpreting the data. For example, the reason that per capita health care expenditure in Finland is 30 per cent lower than in the other countries, may be because the boundary for what is included as health care expenditure on care of the elderly may be different from that in the other countries. At the same time, Table 5.3.3 shows that health care expenditure per capita in Norway is substantially higher than in the other countries. It is important to be aware of the fact that OECD's system of health accounts and EUROSTAT's ESSPROS system are very different. Thus data on health care expenditure from these two sources are very different. EUROSTAT data are published by NOSOSCO in the publication Social Protection in the Nordic Countries.

ESSPROS includes all social arrangements, both public and private. The statistics include pension schemes, insurance schemes, humanitarian organizations and other charitable organizations. Insurance schemes are included if they are collective. This means that expenditure on health also includes sickness benefits (or salary paid during sickness) including sickness benefits paid by employers. These cash payments are not included in OECD's system, in which only expenditure on actual health services is included. 
Table 5.3.1 Total health care expenditure (million national currency) 2014

\begin{tabular}{|c|c|c|c|c|c|c|c|}
\hline & Denmark & $\begin{array}{c}\text { Faroe } \\
\text { Islands }\end{array}$ & $\begin{array}{l}\text { Green- } \\
\text { land }\end{array}$ & Finland $^{1}$ & Iceland & Norway & Sweden \\
\hline & DKK & DKK & DKK & EUR & ISK & NOK & SEK \\
\hline Public financing & 172694 & 1083 & 1285 & 14719 & 142800 & 248490 & 365286 \\
\hline Private financing & 32437 & .. & - & 4804 & 33409 & 43435 & 72844 \\
\hline $\begin{array}{l}\text { Total health care } \\
\text { expenditure }\end{array}$ & 205131 & .. & 1285 & 19528 & 176209 & 291925 & 438130 \\
\hline
\end{tabular}

1 Finnish data include Åland

Source: OECD HEALTH STATISTICS. FO, Statistics Faroe Islands; GL, Directorate of Health

Table 5.3.2 Total health care expenditure (EUR/capita) 2014

\begin{tabular}{|c|c|c|c|c|c|c|c|}
\hline & Denmark & $\begin{array}{c}\text { Faroe } \\
\text { Islands }\end{array}$ & $\begin{array}{c}\text { Green- } \\
\text { land }\end{array}$ & Finland $^{1}$ & Iceland & Norway & Sweden \\
\hline Public financing & 4105 & 2997 & 3062 & 2695 & 2831 & 5790 & 4141 \\
\hline Private financing & 771 & .. & - & 880 & 662 & 1012 & 826 \\
\hline $\begin{array}{l}\text { Total health care } \\
\text { expenditure }\end{array}$ & 4876 & .. & 3062 & 3576 & 3494 & 6802 & 4967 \\
\hline
\end{tabular}

1 Finnish data include Åland

Source: OECD HEALTH STATISTICS. FO, Statistics Faroe Islands; GL, Directorate of Health

Table 5.3.3 GDP and health care expenditure in total and per capita, 2000-2014

\begin{tabular}{|c|c|c|c|c|c|c|c|}
\hline & Denmark $^{1}$ & $\begin{array}{l}\text { Faroe } \\
\text { Islands }^{2}\end{array}$ & Greenland & Finland $^{3}$ & Iceland & Norway & Sweden $^{1}$ \\
\hline & DKK & DKK & DKK & EUR & ISK & NOK & SEK \\
\hline $\begin{array}{l}\text { Total expenditure } \\
\text { per capita } 2014\end{array}$ & 36348 & .. & 22831 & 3576 & 538229 & 56825 & 45186 \\
\hline $\begin{array}{l}\text { GDP (million) } \\
2014\end{array}$ & 1942584 & 15536 & 13701 & 205178 & 2003638 & 3154104 & 3918199 \\
\hline \multicolumn{8}{|l|}{$\begin{array}{l}\text { Expenditure in } \\
2014 \text { prices (mil- } \\
\text { lion) }\end{array}$} \\
\hline 2000 & 143964 & .. & .. & 9353 & 124073 & 194463 & 176433 \\
\hline 2010 & 196172 & 1048 & 1133 & 16618 & 154321 & 260273 & 298721 \\
\hline 2013 & 196187 & 1083 & 1245 & 19327 & 164471 & 274078 & 418514 \\
\hline 2014 & 205131 & .. & 1285 & 19528 & 176209 & 291925 & 438130 \\
\hline \multicolumn{8}{|l|}{$\begin{array}{l}\text { Expenditure as a } \\
\text { percentage of } \\
\text { GDP }\end{array}$} \\
\hline 2000 & 8.1 & 8.5 & 8.9 & 6.7 & 9.0 & 7.7 & 7.4 \\
\hline 2010 & 10.4 & 7.8 & 9.2 & 8.9 & 8.8 & 8.9 & 11.1 \\
\hline 2014 & 10.6 & 7.4 & 9.4 & 9.5 & 8.9 & 9.3 & 11.2 \\
\hline
\end{tabular}

1 Changes in method of calculation from 2003 for Denmark, from 2000 for Norway and from 2001 for Sweden

2 Only public health expenditure

3 Finnish data include Åland

Source: OECD HEALTH STATISTICS. FO, Statistics Faroe Islands; GL, Directorate of Health 
Figure 5.3.1 Total health care expenditure (PPS/capita) in 2015 prices ${ }^{1}$

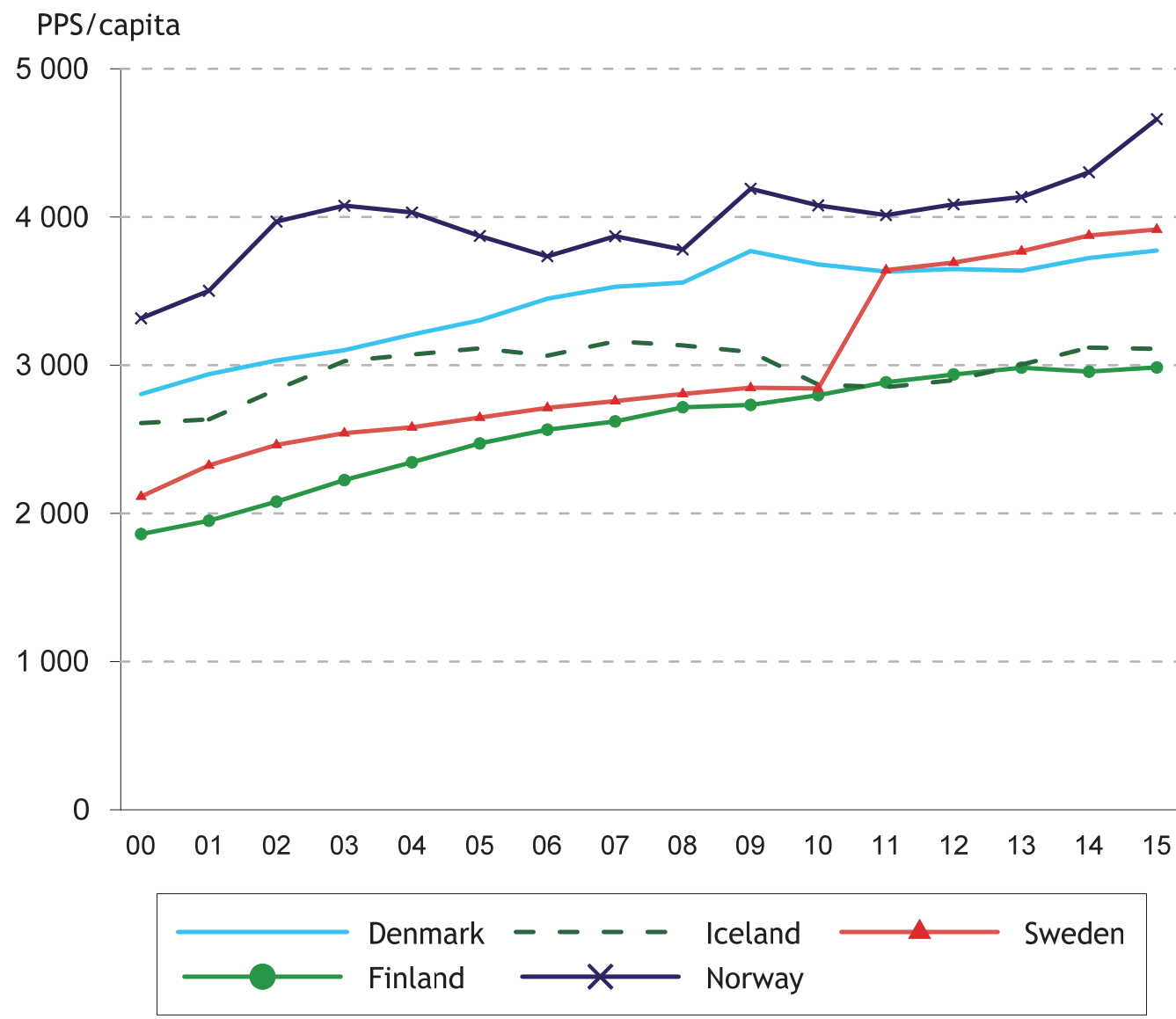

1 PPS, purchasing power standard, is an expression for the different currencies' relative purchasing power

Source: OECD HEALTH STATISTICS 
Figure 5.3.2 Health care expenditure as a percentage of GDP 2000-2015

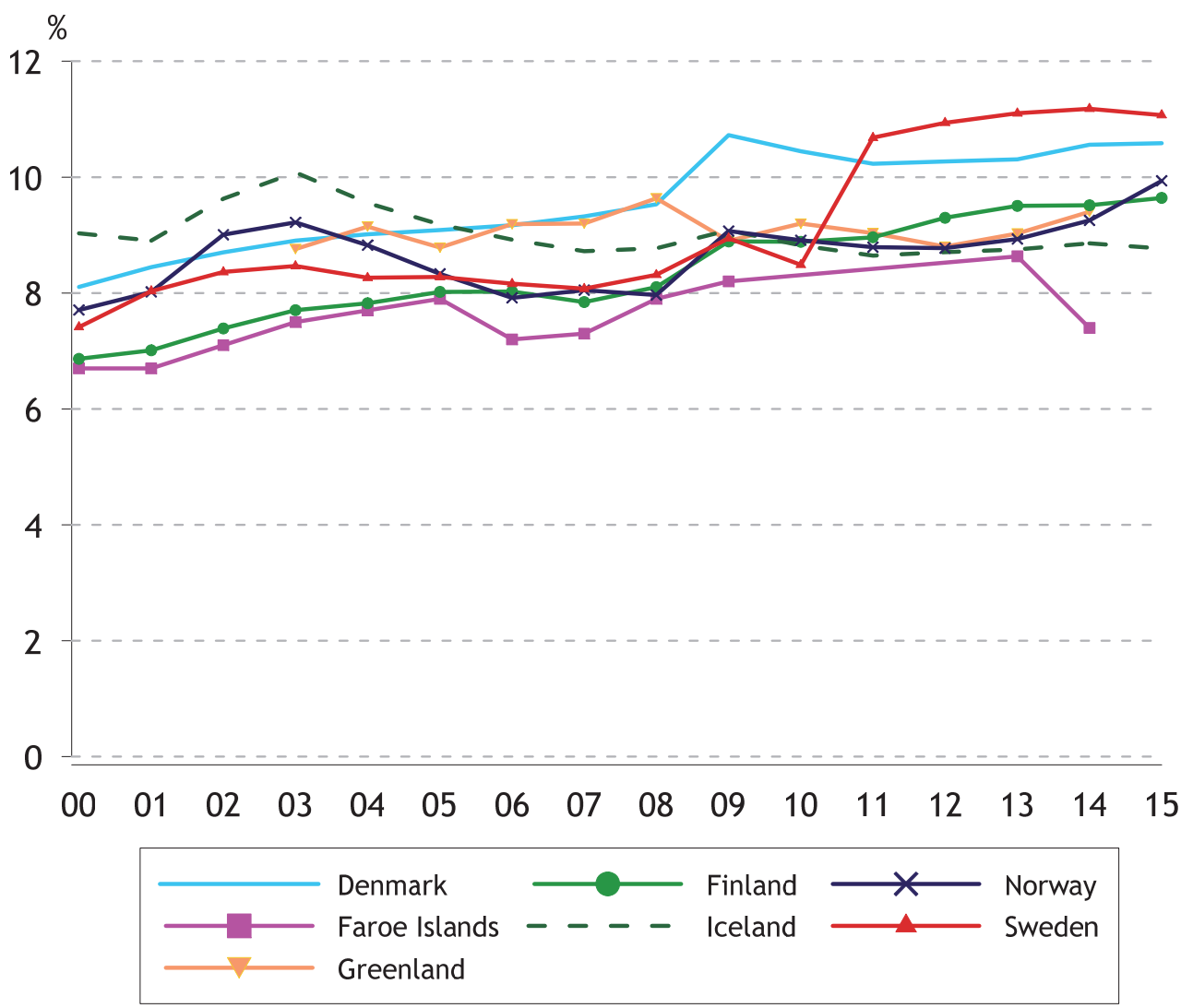

Source: OECD HEALTH STATISTICS; FO, Statistics Faroe Islands; GL, Directorate of Health 


\subsection{Health care personnel}

For many years, it has been difficult to obtain comparable data about health care personnel in the Nordic countries, because the sources for the data have been very different.

Therefore, in 2003, NOMESCO appointed a working group to obtain more comparable data, and to define health care personnel in the way that it is done for health economy in OECD's "A System for Health Accounts".

For this purpose, it has been found to be most appropriate to use NACE's classification of occupations, linked to the registers of authorization for health care personnel. These registers are more comparable, though the data are still incomplete and there are some inaccuracies.

With the new definitions and groups, data on health care personnel for previous years (before 2004) are not comparable with more recent data, since data for new groups of health care personnel are included.

It should be noted that the group 'qualified auxiliary nurses' is now subdivided. Those with an education of at least 18 months remain in this group, while those with an education of less than 18 months are included in the group 'other health care personnel'. Since Sweden only has data for employees in the public service, data for these categories are not included. 'Other health care personnel with a higher education' is defined as personnel with a university degree, such as dieticians and pharmacists. Furthermore, for physicians a group is included with physicians who do not work in the social and health care sectors, and not with medicine.

Besides, the included data are registered at a given time of the year. 
Table 5.4.1 Employed health care personnel in health and social services, 2014 (NACE 85.1 and 85.3)

\begin{tabular}{|c|c|c|c|c|c|c|c|c|}
\hline & Denmark $^{1}$ & $\begin{array}{l}\text { Faroe } \\
\text { Islands }\end{array}$ & Greenland & Finland $^{1}$ & Åland & Iceland $^{2}$ & Norway $^{3}$ & $\begin{array}{c}\text { Sweden } \\
5\end{array}$ \\
\hline Physicians & 20498 & 127 & 98 & 16859 & 87 & 1193 & 22754 & 38429 \\
\hline Dentists & 4336 & 46 & 31 & 3990 & 27 & 274 & 4450 & 7737 \\
\hline Dental hygienists & 1689 & 26 & 47 & 1585 & 8 & 14 & 1031 & 4051 \\
\hline $\begin{array}{l}\text { Dental surgery } \\
\text { assistants }\end{array}$ & 4414 & 83 & 24 & .. & 29 & 321 & 3342 & .. \\
\hline Psychologists & 5489 & 25 & 4 & 3373 & 12 & - & 5086 & 6097 \\
\hline Qualified nurses & 58151 & 366 & 264 & 61309 & 382 & 3032 & 86764 & 102349 \\
\hline Radiographers & 1727 & 5 & .. & 2774 & 9 & 129 & 2907 & 1411 \\
\hline $\begin{array}{l}\text { Qualified } \\
\text { auxiliary nurses }\end{array}$ & 40233 & 103 & 172 & 77012 & 597 & 1987 & 56305 & .. \\
\hline $\begin{array}{l}\text { Other health care } \\
\text { personnel }\end{array}$ & 53986 & & 145 & .. & 43 & - & & .. \\
\hline Midwives & 1916 & 21 & 23 & .. & 17 & 270 & 2843 & 6997 \\
\hline Physiotherapists & 9483 & 18 & 13 & 8389 & 28 & 531 & 9313 & 12111 \\
\hline $\begin{array}{l}\text { Occupational } \\
\text { therapists }\end{array}$ & 6542 & 7 & 2 & .. & 12 & 252 & 3036 & 8289 \\
\hline $\begin{array}{l}\text { Hospital laboratory } \\
\text { technicians }\end{array}$ & 5635 & 38 & 34 & 5438 & 22 & 309 & 4982 & 7944 \\
\hline $\begin{array}{l}\text { Other health care } \\
\text { personnel with a } \\
\text { higher education }\end{array}$ & 675 & - & .. & .. & 36 & - & 6844 & 36203 \\
\hline
\end{tabular}

\section{3}

2 Physicians licensed to practice up to 70 years old at end of year, with permanent residence and registered domicile in Iceland

3 Active health personnel in health and social services

4 The data apply to November

5 An additional 2207 qualified nurses are specialized and employed as radiographers

Source: DK, the Danish Health Data Authority; FO, Ministry of Health Affairs; GL, Chief Medical Officer; FI, THL; ÅL, The Åland Government; IS, Directorate of Health; NO, Statistics Norway; SV, National Board of Health and Welfare 
Table 5.4.2 Employed health care personnel in health and social services per 100000 inhabitants, 2014 (NACE 85.1 and 85.3)

\begin{tabular}{|c|c|c|c|c|c|c|c|c|}
\hline & Denmark $^{1}$ & $\begin{array}{l}\text { Faroe } \\
\text { Islands }\end{array}$ & Greenland & Finland $^{1}$ & Åland & Iceland $^{2}$ & Norway $^{3}$ & $\underset{5}{\text { Sweden }}$ \\
\hline Physicians & 363 & 263 & 173 & 310 & 302 & 366 & 443 & 396 \\
\hline Dentists & 77 & 95 & 55 & 73 & 94 & 84 & 87 & 80 \\
\hline Dental hygienists & 30 & 54 & 83 & 29 & 28 & 4 & 20 & 42 \\
\hline $\begin{array}{l}\text { Dental surgery } \\
\text { assistants }\end{array}$ & 78 & 172 & 43 & .. & 101 & 99 & 65 & - \\
\hline Psychologists & 97 & 52 & 7 & 62 & 42 & - & 99 & 63 \\
\hline Qualified nurses & 1030 & 757 & 467 & 1127 & 1326 & 931 & 1689 & 1056 \\
\hline Radiographers & 31 & 10 & - & 51 & 31 & 40 & 57 & 15 \\
\hline $\begin{array}{l}\text { Qualified auxiliary } \\
\text { nurses }\end{array}$ & 713 & 213 & 304 & 1416 & 2072 & 610 & 1096 & - \\
\hline $\begin{array}{l}\text { Other health } \\
\text { care personnel }\end{array}$ & 957 & - & 257 & .. & 149 & - & - & - \\
\hline Midwives & 34 & 43 & 41 & .. & 59 & 83 & 55 & 72 \\
\hline Physiotherapists & 168 & 37 & 23 & 154 & 97 & 163 & 181 & 125 \\
\hline $\begin{array}{l}\text { Occupational } \\
\text { therapists }\end{array}$ & 116 & 14 & 4 & .. & 42 & 77 & 59 & 85 \\
\hline $\begin{array}{l}\text { Hospital laboratory } \\
\text { technicians }\end{array}$ & 100 & 79 & 60 & 100 & 76 & 95 & 97 & 82 \\
\hline $\begin{array}{l}\text { Other health care } \\
\text { personnel with a } \\
\text { higher education }\end{array}$ & 12 & - & - & .. & 125 & - & 133 & 373 \\
\hline $\begin{array}{ll}1 & 2013 \\
2 & \text { Physicians licens } \\
& \text { tered domicile in } \\
3 & \text { Active health per } \\
4 & \text { The data apply tc } \\
5 & \text { An additional } 22\end{array}$ & $\begin{array}{l}\text { ed to practi } \\
\text { Iceland } \\
\text { rsonnel in he } \\
\text { o November } \\
07 \text { qualified }\end{array}$ & $\begin{array}{l}\text { ce up to } \\
\text { nulth and } \\
\text { nurses a }\end{array}$ & $\begin{array}{l}0 \text { years old } \\
\text { social servic } \\
\text { e specialize }\end{array}$ & $\begin{array}{l}\text { at end of } \\
\text { des and emp }\end{array}$ & red as r & diographe & esidence & nd regis- \\
\hline \multicolumn{9}{|c|}{$\begin{array}{l}\text { Source: DK, the Danish Health Data Authority; FO, Ministry of Health Affairs; GL, Chief Medical Officer; } \\
\text { FI, THL; ÅL, The Åland Government; IS, Directorate of Health; NO, Statistics Norway; SV, Na- } \\
\text { tional Board of Health and Welfare }\end{array}$} \\
\hline
\end{tabular}


Table 5.4.3 Number of general practitioners 2014

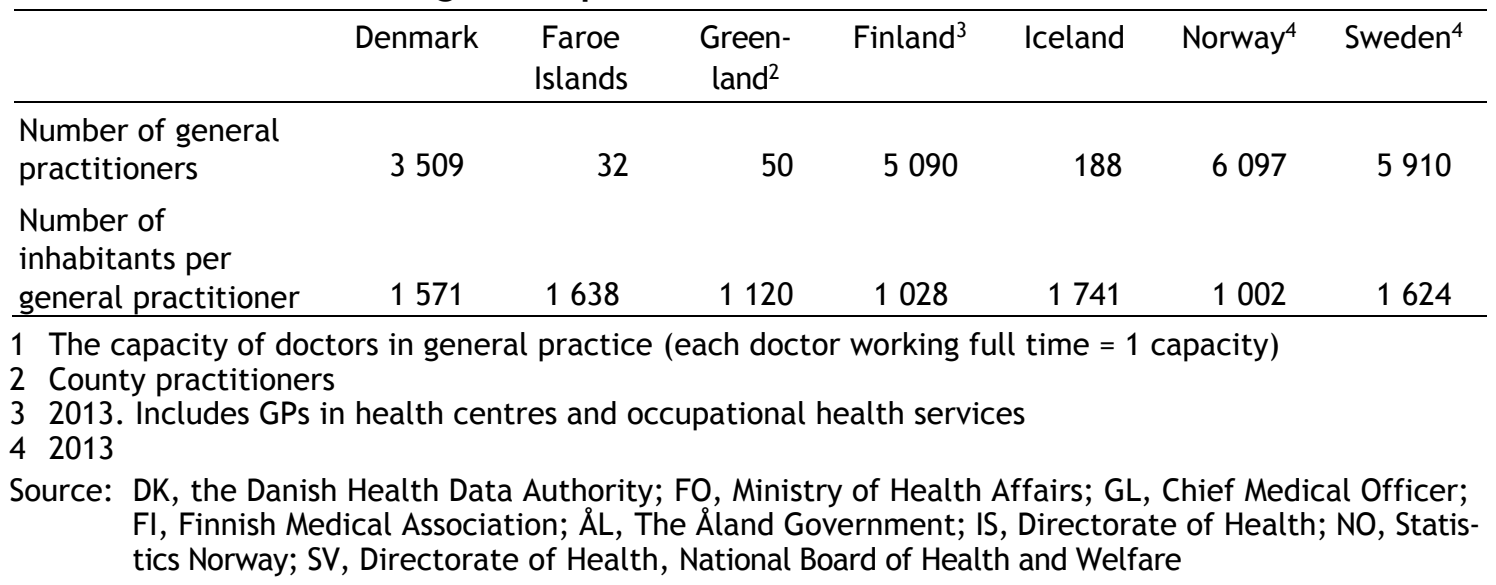


Table 5.4.4 Employed physicians by specialty in health and social services, 2014 (NACE 85.1 and 85.3)

\begin{tabular}{|c|c|c|c|c|c|c|c|c|}
\hline & Denmark $^{1}$ & $\begin{array}{c}\text { Faroe } \\
\text { Islands }^{2}\end{array}$ & $\begin{array}{l}\text { Green- } \\
\text { land }^{3}\end{array}$ & Finland $^{1}$ & Åland & $\underset{5}{\text { Iceland }}{ }^{4}$ & Norway & Sweden $^{6}$ \\
\hline General practice & 4408 & 32 & 53 & 1762 & 16 & 188 & 2763 & 5910 \\
\hline Internal medicine & 1685 & 11 & 4 & 962 & 12 & 159 & 1630 & 1363 \\
\hline Paediatrics & 396 & 2 & 1 & 575 & 3 & 55 & 510 & 983 \\
\hline Surgery & 941 & 7 & 4 & 891 & 4 & 84 & 791 & 1317 \\
\hline Plastic surgery & 112 & 1 & - & 100 & & 10 & 104 & 156 \\
\hline $\begin{array}{l}\text { Gynaecology } \\
\text { and obstetrics }\end{array}$ & 539 & 3 & 3 & 686 & 6 & 45 & 578 & 1339 \\
\hline $\begin{array}{l}\text { Orthopaedic surgery, } \\
\text { incl. hand surgery }\end{array}$ & 716 & 5 & 2 & 473 & 5 & 41 & 521 & 1356 \\
\hline Ophthalmology & 332 & 4 & - & 468 & 2 & 32 & 371 & 707 \\
\hline Ear, nose and throat & 348 & 2 & 1 & 348 & 1 & 21 & 300 & 576 \\
\hline Psychiatry & 997 & 3 & 2 & 1356 & 6 & 75 & 1517 & 1632 \\
\hline $\begin{array}{l}\text { Skin and sexually } \\
\text { transmitted diseases }\end{array}$ & 173 & 2 & - & 197 & & 18 & 158 & 375 \\
\hline Neurology & 311 & 1 & - & 482 & & 17 & 301 & 394 \\
\hline Oncology & 158 & & - & 162 & 1 & 13 & 212 & 436 \\
\hline Anaesthetics & 998 & 5 & 4 & 786 & 4 & 60 & 828 & 1622 \\
\hline Radiology & 523 & 4 & 2 & 681 & 2 & 33 & 688 & 1104 \\
\hline $\begin{array}{l}\text { Clinical laboratory } \\
\text { specialities } \\
\text { incl. pathology }\end{array}$ & 551 & 1 & - & 185 & & 34 & 449 & 938 \\
\hline Other specialities & 140 & 2 & & 1701 & 10 & 25 & 570 & 6255 \\
\hline Specialists in total & 13328 & 83 & 76 & 11815 & 72 & 910 & 12291 & 26463 \\
\hline $\begin{array}{l}\text { Physicians } \\
\text { without specialist } \\
\text { authorization }\end{array}$ & 7170 & 49 & 22 & 5044 & 14 & 283 & 10463 & 11966 \\
\hline $\begin{array}{l}\text { Physicians in total } \\
\text { within NACE } 85.1 \text { and } \\
85.3\end{array}$ & 20498 & 132 & 98 & 16859 & 86 & 1193 & 22754 & 38429 \\
\hline
\end{tabular}

12013

2 Full-time equivalents, of which 11 specialists had full-time positions as consultants. The figure for 2013 is not comparable with the figure for 2012. The number of specialist consultants was too low in the last report. The number of physicians without specialization (specifically general practice trainee) was 6 full-time equivalents too low last year

3 Physicians working as general practitioners, but some of them might have other specialities. A few (6) have surgical skills to be able to perform a Caesarean section

4 Data based on the register of physicians at the Directorate of Health. The most recent specialty is chosen for those with more than one specialty

5 Physicians licensed to practice in Iceland, up to the age of 70 years at the end of the year, with permanent residence and registered domicile in Iceland

6 The data apply to November

Source: DK, the Danish Health Data Authority; FO, Ministry of Health Affairs; GL, Chief Medical Officer; FI, Finnish Medical Association; ÅL, The Åland Government; IS, Directorate of Health; NO, Statistics Norway; SV, National Board of Health and Welfare 
Table 5.4.5 Number of employed physicians by specialty in health and social services per 100000 inhabitants, 2014 (NACE 85.1 and 85.3)

\begin{tabular}{|c|c|c|c|c|c|c|c|c|}
\hline & Denmark $^{1}$ & $\begin{array}{l}\text { Faroe } \\
\text { Islands }^{2}\end{array}$ & $\begin{array}{c}\text { Green- } \\
\text { land }^{3}\end{array}$ & Finland $^{5}$ & Åland & $\begin{array}{l}\text { Iceland }_{5}^{4} \\
\end{array}$ & Norway & Sweden $^{6}$ \\
\hline General practice & 78 & 66 & 94 & 33 & 56 & 58 & 54 & 61 \\
\hline Internal medicine & 30 & 23 & 7 & 18 & 42 & 49 & 32 & 14 \\
\hline Paediatrics & 7 & 5 & 2 & 11 & 10 & 17 & 10 & 10 \\
\hline Surgery & 17 & 14 & 7 & 16 & 14 & 26 & 15 & 14 \\
\hline Plastic surgery & 2 & 1 & - & 2 & - & 3 & 2 & 2 \\
\hline $\begin{array}{l}\text { Gynaecology } \\
\text { and obstetrics }\end{array}$ & 10 & 5 & 5 & 13 & 21 & 14 & 11 & 14 \\
\hline $\begin{array}{l}\text { Orthopaedic surgery, } \\
\text { incl. hand surgery }\end{array}$ & 13 & 9 & 4 & 9 & 17 & 13 & 10 & 14 \\
\hline Ophthalmology & 6 & 8 & - & 9 & 7 & 10 & 7 & 7 \\
\hline Ear, nose and throat & 6 & 4 & 2 & 6 & 3 & 6 & 6 & 6 \\
\hline Psychiatry & 18 & 6 & 4 & 25 & 21 & 23 & 30 & 17 \\
\hline $\begin{array}{l}\text { Skin and sexually } \\
\text { transmitted diseases }\end{array}$ & 3 & 4 & 0 & 4 & 0 & 6 & 3 & 4 \\
\hline Neurology & 6 & 1 & 0 & 9 & 0 & 5 & 6 & 4 \\
\hline Oncology & 3 & 0 & 0 & 3 & 3 & 4 & 4 & 4 \\
\hline Anaesthetics & 18 & 11 & 7 & 15 & 14 & 18 & 16 & 17 \\
\hline Radiology & 9 & 7 & 4 & 13 & 7 & 10 & 13 & 11 \\
\hline $\begin{array}{l}\text { Clinical laboratory } \\
\text { specialities } \\
\text { incl. pathology }\end{array}$ & 10 & 2 & - & 3 & - & 10 & 9 & 10 \\
\hline Other specialities & 2 & 4 & - & 31 & 35 & 8 & 11 & 65 \\
\hline Specialists in total & 236 & 171 & 134 & 218 & 250 & 279 & 239 & 273 \\
\hline $\begin{array}{l}\text { Physicians } \\
\text { without specialist } \\
\text { authorization }\end{array}$ & 127 & 102 & 39 & 93 & 49 & 87 & 204 & 123 \\
\hline $\begin{array}{l}\text { Physicians in total } \\
\text { within NACE } 85.1 \text { and } \\
85.3\end{array}$ & 363 & 273 & 173 & 311 & 298 & 366 & 443 & 396 \\
\hline
\end{tabular}

12013

2 Full-time equivalents, of which 11 specialists had full-time positions as consultants. The figure for 2013 is not comparable with the figure for 2012. The number of specialist consultants was too low in the last report. The number of physicians without specialization (specifically general practice trainee) was 6 full-time equivalents too low last year

3 Physicians working as general practitioners, but some of them might have other specialities. A few (6) have surgical skills to be able to perform a Caesarean section

4 Data based on the register of physicians at the Directorate of Health. The most recent specialty is chosen for those with more than one specialty

5 Physicians licensed to practice in Iceland, up to the age of 70 years at the end of the year, with permanent residence and registered domicile in Iceland

6 The data apply to November

Source: DK, the Danish Health Data Authority; FO, Ministry of Health Affairs; GL, Chief Medical Officer; FI, Finnish Medical Association; ÅL, The Åland Government; IS, Directorate of Health; NO, Statistics Norway; SV, National Board of Health and Welfare 
Table 5.4.6 Employed physicians 2014

\begin{tabular}{|c|c|c|c|c|c|c|c|c|}
\hline & Denmark $^{1}$ & $\begin{array}{l}\text { Faroe } \\
\text { Islands }\end{array}$ & $\begin{array}{l}\text { Green- } \\
\text { land }\end{array}$ & Finland $^{1}$ & Åland ${ }^{1}$ & Iceland & Norway & Sweden \\
\hline $\begin{array}{l}\text { Physicians employed } \\
\text { in hospitals } \\
\text { (NACE } 85.1 \text { and } 85.3 \text { ) }\end{array}$ & 14234 & 97 & 98 & 7800 & 59 & 883 & .. & .. \\
\hline $\begin{array}{l}\text { General practitioners } \\
\text { (NACE } 85.1 \text { and } 85.3 \text { ) }\end{array}$ & 4539 & 30 & .. & 5650 & 14 & .. & 12137 & 5910 \\
\hline $\begin{array}{l}\text { of whom working } \\
\text { without specialist } \\
\text { authorization }\end{array}$ & 395 & $49^{2}$ & .. & 3273 & .. & .. & 6318 & - \\
\hline $\begin{array}{l}\text { Other physicians } \\
\text { working outside hos- } \\
\text { pitals (mainly } \\
\text { privately practising } \\
\text { specialists) (NACE } \\
85.1 \text { and } 85.3 \text { ) }\end{array}$ & 1725 & .. & .. &.. & 13 & .. & 4299 & .. \\
\hline $\begin{array}{l}\text { Physicians employed } \\
\text { in administrative } \\
\text { medicine (NACE 75.1) }\end{array}$ & 252 & 1 & .. & .. & 2 & .. & 1594 & 821 \\
\hline $\begin{array}{l}\text { Physicians employed } \\
\text { in medical research, } \\
\text { teaching etc. (NACE } \\
80.3,73.1 \text { and } 24.4 \text { ) }\end{array}$ & 1016 & 1 & .. & .. & .. & .. & 467 & 1199 \\
\hline $\begin{array}{l}\text { Physicians employed } \\
\text { within all other } \\
\text { NACE codes }\end{array}$ & 958 & - & .. & .. & .. & .. & 1203 & 1052 \\
\hline
\end{tabular}

12013

Source: DK, the Danish Health Data Authority; FO, Ministry of Health Affairs; GL, Chief Medical Officer; FI, Finnish Medical Association; ÅL, The Åland Government; IS, Directorate of Health; NO, Statistics Norway; SV, National Board of Health and Welfare 
Ressourcer

Table 5.4.7 Foreign-trained physicians and nurses - Stock and in per cent

\begin{tabular}{|c|c|c|c|c|}
\hline & Denmark & Finland & Norway & Sweden \\
\hline \multicolumn{5}{|c|}{ Foreign-trained physicians - Stock } \\
\hline 2000 & 681 & .. & .. & 3827 \\
\hline 2005 & 1092 & .. & .. & 5866 \\
\hline 2010 & 1158 & 3528 & 6766 & 8552 \\
\hline 2013 & .. & .. & 7808 & .. \\
\hline 2014 & .. &.. & 8447 & .. \\
\hline \multicolumn{5}{|c|}{$\%$ of foreign-trained physicians } \\
\hline 2000 & 4.4 &.. & .. & .. \\
\hline 2005 & 6.1 &.. & .. & .. \\
\hline 2010 & 5.9 & 20.9 & 33.8 & 13.9 \\
\hline 2013 & .. & .. & 35.8 & 18.4 \\
\hline 2014 & .. & .. & 37.3 & 23.4 \\
\hline \multicolumn{5}{|c|}{ Foreign-trained nurses - Stock } \\
\hline 2000 & 889 &.. & .. & 2358 \\
\hline 2005 & 817 &.. & .. & 2796 \\
\hline 2010 & 751 & 910 & 5940 & 2858 \\
\hline 2013 & .. &.. & 7191 & .. \\
\hline 2014 & .. &.. & 7640 & .. \\
\hline \multicolumn{5}{|c|}{$\%$ of foreign-trained nurses } \\
\hline 2000 & 1.8 & .. & .. & 2.7 \\
\hline 2005 & 1.6 & .. &.. & 2.9 \\
\hline 2010 & 1.4 & 1.3 & 7.9 & 2.8 \\
\hline 2013 & .. & .. & 8.8 & .. \\
\hline 2014 &.. &.. & 9.1 & .. \\
\hline
\end{tabular}

Source: OECD HEALTH STATISTICS 


\subsection{Capacity and services in the hospital sector}

For many years, there has been a trend in the Nordic countries towards fewer hospital beds. Resources have been concentrated in fewer units, often involving a division of work in the most specialized areas. Units have often been merged administratively, not necessarily leading to fewer physical units. No hospitals have been closed down in Norway during the last few years, but some of the existing hospitals have become smaller.

Another trend in the Nordic countries is that psychiatric hospitals are being closed down, but to a varying degree.

However, the structure is somewhat different in Finland, Iceland and Greenland than in the other countries. A number of beds are attached to health centres, and these beds appear in the tables as beds in "other hospitals". Some of these beds are for care of elderly people, and they are similar to beds in nursing homes and old peoples' homes in the other countries. Particularly for Finland and Iceland, this gives a larger number of beds in relation to the population than in the other countries.

Hospital beds are divided into medical, surgical, psychiatric and other beds. Particularly for Finland and Iceland, the category 'other', includes activities that are not included in the other countries.

The tables on hospital discharges and average length of stay apply to patients admitted to ordinary hospitals and specialized hospitals. This limitation has been done in order to improve comparability between the countries.

The trend is that the number of treatment places and the average length of stay have been reduced in ordinary hospitals. Within mental health care treatment, there has been a trend towards the use of more out-patient treatment, for which reason the number of psychiatric beds has been reduced. 
Table 5.5.1 Available hospital beds by speciality, 2014

\begin{tabular}{|c|c|c|c|c|c|c|c|c|}
\hline & Denmark $^{1}$ & $\begin{array}{l}\text { Faroe }^{2} \\
\text { Islands }\end{array}$ & Greenland $^{3}$ & Finland $^{3}$ & Åland ${ }^{2,3}$ & Iceland $^{4}$ & Norway $^{5}$ & Sweder \\
\hline \multicolumn{9}{|l|}{ Number } \\
\hline Somatic wards & 12142 & 193 & 104 & 8645 & 66 & 747 & 11665 & 20235 \\
\hline Psychiatry wards & 1618 & 55 & 12 & 3318 & 13 & 145 & 5968 & 4369 \\
\hline Other & .. & . & 348 & 12795 & 35 & 149 & 2118 & .. \\
\hline Total & 13760 & 248 & 464 & 24758 & 114 & 1041 & 19751 & 24604 \\
\hline \multicolumn{9}{|l|}{$\begin{array}{l}\text { Beds per } 100000 \\
\text { inhabitants }\end{array}$} \\
\hline Somatic wards & 215 & 400 & 184 & 158 & 227 & 228 & 227 & 208 \\
\hline Psychiatry wards & 29 & 114 & 21 & 61 & 44 & 44 & 116 & 45 \\
\hline Other & .. & . & 616 & 234 & 123 & 46 & 41 & .. \\
\hline Total & 244 & 514 & 821 & 453 & 394 & 318 & 385 & 254 \\
\hline
\end{tabular}

1 Total number of available beds reported by hospitals/regions per December 2014. Not all psychiatric beds registered for 2014 due to a new reporting method

2 2010-14

3 Number of bed-days divided by 365

4 Other beds are beds for long-term care in hospitals (health facilities with 24-hour access to hospital physicians)

5 Figures include beds within mental health care services and substance abuse treatment. This includes both beds in hospitals and community mental health care centres (DPS)

Source: DK, the Danish Health Data Authority; FO, Ministry of Health Affairs; GL, Chief Medical Officer; FI, THL; ÅL, The Åland Government; IS, Directorate of Health; NO, Statistics Norway; SV, National Board of Health and Welfare 
Appendix 


\section{Further Information on the Bodies Responsible for Statistics in the Nordic Countries}

The following bodies responsible for statistics in the Nordic countries can be contacted for further information concerning the statistics in this publication.

\section{Denmark}

Statistics Denmark

www.dst.dk

The Danish Health Data Authority www.sundhedsdatastyrelsen.dk

National Board of Health

www.sst.dk

Responsible for:

- Population statistics

- Statistics on alcohol consumption

- Statistics on health care economy

Responsible for:

- Statistics on births

- Statistics on induced abortions

- Statistics on congenital anomalies

- Statistics on causes of death

- Statistics on hospital services

- Statistics on health care personnel

- Statistics on infectious diseases

- Statistics and information on vaccinations

- Statistics on pharmaceutical products

Responsible for:

- Statistics on the use of tobacco

\section{Faroe Islands}

Statistics Faroe Islands

www. hagstova.fo

Chief Medical Officer

www.landslaeknin.fo

Responsible for:

- Population and vital statistics

Responsible for:

- Statistics on infectious diseases

- Statistics on forensics

- Statistics on births

- Statistics on causes of death

Chief Pharmaceutical Officer

www.apotek.fo

Ministry of Health and the Interior www.himr.fo

The Danish Health Data Authority www.sundhedsdatastyrelsen.dk

Responsible for:

- Statistics on pharmaceutical products

Responsible for:

- Statistics on health care personnel

- Statistics on hospital services

- Statistics on induced abortions

- Statistics and information on vaccinations

Responsible for:

- Statistics on causes of death

- Statistics on health care economy 


\section{Greenland}

Statistics Greenland

www.stat.gl

National Board of Health

www.nun.gl

E-mail: nun@nanoq.gl

The Danish Health Data Authority

www.sundhedsdatastyrelsen.dk

Chief Pharmaceutical Officer

www.peqqik.gl

E-mail: apotek@peqqik.gl

The Department of Health and Infrastructure
Responsible for:

- Population and vital statistics

- Statistics on health care personnel

- Statistics on hospital services

- Statistics on health care economy

Responsible for:

- Statistics on births

- Statistics on induced abortions

- Statistics on congenital anomalies

- Statistics on infectious diseases

- Statistics and information on vaccinations

Responsible for:

- Statistics on causes of death

- Statistics on cancer

Responsible for:

- Statistics on pharmaceutical products

Responsible for:

- Statistics on hospital services

- Statistics on health care economy

- Statistics on health care personnel

\section{Finland}

Statistics Finland

www.stat.fi

National Institute for Health and Welfare www.thl.fi
Responsible for:

- Population and vital statistics

- Statistics on causes of death

- Statistics on road traffic accidents

- Statistics on income and living conditions (EU-SILC)

Responsible for:

- Statistics on institutional care

- Statistics on births

- Statistics on congenital anomalies

- Statistics on induced abortions and sterilizations

- Statistics on health care personnel

- Statistics on public health care

- Statistics on private health care

- Statistics on labour force in health care

- Statistics on the use of alcohol and drugs 
National Agency for Medicines (FIMEA) www.fimea.fi

Social Insurance Institution of Finland (FPA) www.kela.fi

The Cancer Register www.cancer.fi

Finnish Centre for Pensions (ETK) www.etk.fi

\section{Åland}

The Åland Government www.regeringen.ax

Statistics Finland

National Institute for Health and Welfare National Agency for Medicines Finnish Cancer Registry Social Insurance Institution of Finland Finnish Centre for Pensions
- Statistics on the use of tobacco

- Statistics on health care expenditure

- Definitions and classifications in health care

- Statistics on primary health care

- Statistics on hospital care and surgery

- Statistics on infectious diseases

- Statistics and information on vaccinations

- Health interview and examination surveys

- Public Health Report

Responsible for:

- Registration of pharmaceutical products and sales licences

- Statistics on adverse drug reactions

- Statistics on pharmacies

Responsible for:

- Sickness insurance benefits and allowances, reimbursements for medicine expenses for pharmaceutical products, and disability pensions

Responsible for:

- Statistics on cancer and cancer screening

Responsible for:

- Statistics on pensions due to reduced capacity for work

Responsible for:

- Statistics on health care personnel

- Statistics on hospital services, such as capacity (number of beds)

- Statistics on health care economy user charges for health care

See Finland 


\section{Iceland}

Statistics Iceland

www.statice.is

Directorate of Health

www.landlaeknir. is

Icelandic Medicines Agency

www.imca.is

Icelandic Cancer Society

www.krabb.is

\section{Norway}

Statistics Norway

www.ssb.no

Norwegian Institute of Public Health www.fhi.no

Norwegian Directorate of Health www. helsedirektoratet.no
Responsible for:

- Population and vital statistics

- Statistics on causes of death

- Statistics on alcohol consumption

- Statistics on health care expenditure

- National accounts

Responsible for:

- Medical statistics on births

- Statistics on induced abortions

- Statistics on sterilizations

- Statistics on primary health care

- Statistics on hospital services

- Statistics on infectious diseases

- Statistics on vaccinations

- Statistics on health care personnel

- Statistics on use of tobacco

Responsible for:

- Statistics on pharmaceutical products

Responsible for:

- Statistics on cancer

Responsible for:

- Population and vital statistics

- Statistics on health and social conditions

- Statistics on health and social services

- Statistics on health care personnel

- Statistics on alcohol consumption

- Statistics on health care economy

- Statistics on use of tobacco

Responsible for:

- Statistics on sexually transmitted diseases and infectious

- Statistics on tuberculosis

- Statistics on immunization

- Statistics on sale of pharmaceutical products

- Statistics on prescription drugs

- Statistics on births and infant deaths

- Statistics on induced abortions

- Statistics on causes of death

Responsible for:

- Statistics on hospital services 
Cancer Registry of Norway

www.kreftregisteret.no

Ministry of Health and Care Services www.regjeringen.no/en/dep/hod

\section{Sweden}

Statistics Sweden

www.scb.se

National Board of Health and Welfare www.socialstyrelsen.se

Public Health Agency of Sweden www.folkhalsomyndigheten.se

Swedish Association of Local Authorities and Regions

www.skl.se
Responsible for:

- Statistics on cancer

Responsible for:

- Statistics on in vitro fertilization

Responsible for:

- Population and vital statistics

- Statistics on health care economy

- Study on Living Conditions (ULF/SILC)

Responsible for:

- Statistics on births

- Statistics on induced abortions

- Statistics on in-patients

- Statistics on cancer

- Statistics on causes of death

- Statistics on prescription drugs

- Statistics on authorized health personnel

Responsible for:

- Statistics on infectious diseases

- Statistics and information on vaccinations

- Statistics on alcohol abuse

Responsible for:

- Statistics on health personnel

- Statistics on hospital capacity

- Statistics on health economics 


\section{NOMESCO's Publications since 2000}

\section{Recurring Publications}

Each year, NOMESCO publishes Health Statistics in the Nordic Countries. Up until and including 2011, this was a bi-lingual publication in Danish (Nordic languages) and English.

In cooperation with the Nordic Centre for Classification of Health Services (Nordclass), NOMESCO publishes NOMESCO Classification of Surgical Procedures. The publication has been updated annually for a number of years and is now available in version 1.16.

In cooperation with the Baltic countries, the publication Nordic/Baltic Health Statistics has been published four times, the latest version with data from 2006.

Moreover, a number of theme publications have been published. These are shown below with their number in NOMESCO's publication list.

99. Financing of Health Care in the Nordic Countries, 2013

92. NOMESCO Report on Mortality Statistics - Theme section 2010, NOMESCO, Copenhagen 2010

90. Temasektion vedrørende kvalitetsindikatorer, NOMESCO's Health Statistics in the Nordic Countries 2009, NOMESCO, Copenhagen 2010

88. Medicines Consumption in the Nordic Countries 2004-2008. NOMESCO, Copenhagen 2010

82. Eldres Helse, Temasektion, Health Statistics in the Nordic Countries 2006

80. Mental Helse, Temasektion, Health Statistics in the Nordic Countries 2005

79. NOMESCO Classification of External Causes of Injuries. Fourth revised edition. NOMESCO, Copenhagen 2007

78. Sustainable Social and Health Development in the Nordic Countries. Seminar, 6th April 2006, Oslo. Seminar Report. NOMESCO, Copenhagen 2006

76. Smedby, Björn and Schiøler Gunner: Health Classifications in the Nordic Countries. Historic development in a national and international perspective 2006. NOMESCO, Copenhagen 2006

72. Medicines Consumption in the Nordic Countries 1999-2003. NOMESKO, Copenhagen 2004

75. Patienter I Öppen Värd, Temasektion, Health Statistics in the Nordic Countries 2004

73. Barns Helse, Temasektion, Health Statistics in the Nordic Countries 2003

69. Vård på lika villkor, Temasektion, Health Statistics in the Nordic Countries 2002 
66. Validitet och jämförbarhet i NOMESKO:s dagkirurgistatistik, Section B, Health Statistics in the Nordic countries 2001

67. Sustainable Social and Health Development in the Nordic Countries. Seminar 27th May 2003, Stockholm. NOMESCO, Copenhagen 2003

64. Validitet og sammenlignbarhet av statistikk over kirurgiske inngrep ved nordiske sykehus, Temasektion, Health Statistics in the Nordic countries 2000

58. Nordiske læger og sygeplejersker med autorisation i et andet nordisk land. Copenhagen 2000 